Klaus Günther

\title{
Das Hirn \\ der Studierenden
}

Dialogisches Lernen

statt obrigkeitlicher Lehre 
Klaus Günther

Das Hirn der Studierenden 
Klaus Günther

\section{Das Hirn der Studierenden}

Dialogisches Lernen statt obrigkeitlicher Lehre

Verlag Barbara Budrich

Opladen • Berlin • Toronto 2019 
Bibliografische Information der Deutschen Nationalbibliothek

Die Deutsche Nationalbibliothek verzeichnet diese Publikation in der Deutschen

Nationalbibliografie; detaillierte bibliografische Daten sind im Internet über

http://dnb.d-nb.de abrufbar.

(C) 2019 Dieses Werk ist beim Verlag Budrich UniPress erschienen und steht unter der Creative Commons Lizenz Attribution-ShareAlike 4.0 International (CC BY-SA 4.0): https://creativecommons.org/licenses/by-sa/4.0/

Diese Lizenz erlaubt die Verbreitung, Speicherung, Vervielfältigung und Bearbeitung bei Verwendung der gleichen CC-BY-SA 4.0-Lizenz und unter Angabe der UrheberInnen, Rechte, Änderungen und verwendeten Lizenz.

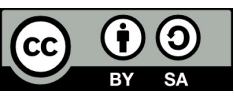

Dieses Buch steht im Open-Access-Bereich der Verlagsseite zum kostenlosen Download bereit (https://doi.org/10.3224/84742206).

Eine kostenpflichtige Druckversion kann über den Verlag bezogen werden. Die Seitenzahlen in der Druck- und Onlineversion sind identisch.

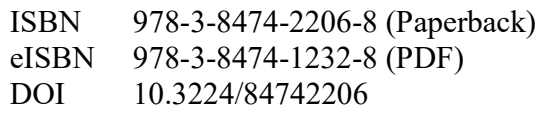

Umschlaggestaltung: Bettina Lehfeldt, Kleinmachnow - www.lehfeldtgraphic.de Titelbildnachweis: Florian Losch

Lektorat: Dr. Andrea Lassalle, Berlin - andrealassalle.de

Satz: Linda Kutzki, Berlin - www.textsalz.de

Druck: Paper \& Tinta, Warschau 
Inhaltsverzeichnis

Für eilige Leserinnen und Leser $\quad$................................................................. 7

Einführung

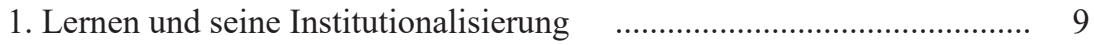

2. Lernen in individueller Kommunikation mit Menschen und (Lern-) Materialien - Nahkommunikation und Fernkommunikation $\quad$............ 11

3. Diffuses Unbehagen an obrigkeitlicher Lehre und seine Beschwichtigung ................................................................... 12

4. Die Herausforderungen der Neurobiologie $\quad$........................................ 15

5. Lernen in Nahkommunikation an Schulen: Von intuitiver zu neurobiologisch informierter Pädagogik und Didaktik f.................... 17

6. Neurobiologisch informiertes Lernen an Hochschulen gegen obrigkeitliche Lehre - zur Gliederung ….......................................... 21

I. Der Kontext des Lernens in Geschichte und Gegenwart .............. 23

1. Zur pädagogisch-didaktischen Indifferenz im Obrigkeitsstaat $\quad$.......... 23

2. Die Fortsetzung obrigkeitlicher Lehre in der Bundesrepublik $\quad$.......... 26

II. Neurobiologische Grundlagen des Lernens $\quad$................................. 31

1. Grundkenntnisse über den neuronalen Lernapparat $\quad$....................... 32

2. Ertragreiches Lernen durch emotionale Schubkräfte in Nahkommunikation …............................................................... 40

3. Lernen in Machtbeziehungen $\quad$........................................................ 45

III. Allgemeine Lern-Konstellation $\quad$......................................................... 51

1. Lernende im Macht-Diskurs (=Lern-Diskurs) …........................... 52

2. Zur Neurobiologie der Winzigkeit $\quad$................................................. 59

IV. Spezielle Lern-Konstellation im Vorlesungsbetrieb $\quad \ldots . . . . . . . . . . . . . . . . \quad 71$

1. Vorlesungs-Idealtypen …........................................................ 72

2. Vorlesungs-Bewertung …………………........................................ 74

3. Neurobiologisch informierte Argumente gegen den Instruktions-Anspruch von (Massen-)Vorlesungen $\quad$........................... 82

V. Neurobiologisch informiertes dialogisches Lernen - traditionell und

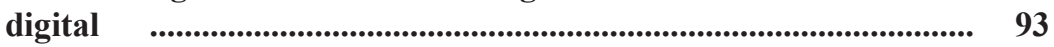

1. Synaptische Grundlegung dialogischen Lernens $\quad$.............................. 94

2. Grundlagen eines Konzepts dialogischen Lernens $\quad$....................... 100 
3. Zum Einsatz digitaler Lernmaterialien

4. Digitales Präsenz-Lernen im Rahmen des Konzepts dialogischen Lernens

5. Wer dialogisches Präsenz-Lernen anleiten soll

6. Die Chance der Hochschulen als Ort dialogischen Lernens

Schlussbilanz

1. Obrigkeitliche Lern-Konstellation und dialogische Lern-Konstellation

2. Ertragarme obrigkeitlicher Lehre an bundesrepublikanischen Hochschulen

3. Wie in dialogischer Lern-Konstellation hoher Lernertrag entsteht ....

4. Zum pädagogisch-didaktischen Mehrwert neurobiologisch informierten Lernens

Exkurs 1

Exkurs 2

Exkurs 3

Literaturverzeichnis

Theorie und Methodologie der modernen Gesellschaft $\quad \ldots \ldots \ldots \ldots \ldots \ldots \ldots \ldots . . . . . . . . . . .143$

Prinzipien der Neurobiologie

Theorie und Empirie des Lernens und seine neurobiologischen

Grundlagen

Historische Entwicklung der deutschen Universitäten

Studentische Erfahrungen an Hochschulen und Hochschuldidaktik $\quad$...... 147

Personenregister

Sachregister 


\section{Für eilige Leserinnen und Leser}

In einer Tradition bekennender pädagogisch-didaktischer Indifferenz hat sich an Hochschulen bis heute obrigkeitliche Lehre behaupten können. Sie lässt sich aus neurobiologischer Sicht folgendermaßen charakterisieren:

Nach einem objektivistisch-empiristischen Modell des Lernens gleichen menschliche Gehirne Wachs- oder Schiefertafeln, auf denen Lernimpulse aufgezeichnet werden. Das gilt speziell für das Wissen, das diejenigen, die das Lernen anleiten, kraft ihrer Amtsautorität eins zu eins an die Lernenden weiterzugeben suchen.

Wenn diesem vorlesungsorientierten Frontalismus des Lernens elementare Kenntnisse über Abläufe in menschlichen Gehirnen und spezielle Kenntnisse zur Gedächtnisbildung entgegengesetzt werden, so bedeutet das nicht, dass die ins Spiel gebrachte Neurobiologie den Anspruch erheben könnte, eigenständig Lernkonzepte zu entwickeln. Es heißt aber, dass die Neurobiologie das Potential hat, als pädagogisch-didaktische Prüfungsinstanz zu dienen und obrigkeitlicher Lehre das Folgende entgegenhalten kann:

Obrigkeitliche Lehre ist unzulänglich auf das Fassungsvermögen des ebenso eigenständig wie eigensinnig arbeitenden Lernapparats menschlicher Gehirne abgestimmt. Der „frontalistische Zugriff“ ist nicht geeignet, alle Sinne herauszufordern. Er erreicht das vielfach ausdifferenzierte neuronale Netzwerk nicht in seiner Breite, Dichte und Tiefe. Es mangelt ihm an Nahkommunikation und an empathischer Nähe. Insgesamt werden zu wenig emotionale Schubkräfte erzeugt, von denen die Übertragung des Inputs vom Kurzzeitgedächtnis auf das Langzeitgedächtnis und damit der Lernerfolg abhängt.

Diese Einsicht muss als Voraussetzung dafür angesehen werden, dass die vielfältigen Vorschläge, die auf ,,anderes Lernen“ gerichtet sind, größere Durchschlagskraft gewinnen. Dank ihrer immer wieder neu zu erprobenden Gehirngerechtigkeit gehören sie ins Zentrum des Lernbetriebs der Hochschulen. Es lassen sich die wichtigsten Komponenten eines Konzepts dialogischen Lernens beschreiben, das den Einsatz digitaler Lernmaterialien einschließt. 



\section{Einführung}

Bert Brecht gelingt in Szene 6 seines „Leben des Galilei“ (1938/1939) eine spektakuläre Illustration ,hinterwäldlerischer“ hoher Geistlicher, Mönche und Gelehrter. Sie arbeiten im Forschungsinstitut des Vatikans, dem Collegium Romanum, und in ihren Augen ist die kopernikanische Wende, nach der sich die Erde um die Sonne dreht, ein Ding der Unmöglichkeit. Sie versuchen die vom anwesenden Galilei belegte Einsicht zu karikieren, indem sie sich unter Gelächter auf der für sie festgefügten und im Zentrum der Welt stehenden Erde wie schwankende Betrunkene bewegen.

An Hochschulen kommt es nicht selten vor, dass sowohl diejenigen, die das Lernen anleiten, als auch die Studierenden insgeheim oder auch ausdrücklich über Veranstaltungsformen spotten, die nicht ins Muster des traditionellen „Frontalismus der Lehre“ passen. Das gilt zum Beispiel für die Physikschau von Physikern, die ihre Erfahrungen dazu gebracht haben, den schwierigen Stoff ihres Faches - ohne dabei unseriös vereinfachend vorzugehen - in spielerischem Format darzubieten. Fundieren lassen sich solche lernintensiven Erfahrungen durch eine Neurobiologie, die mithilfe neuer technischer Verfahren zumindest teilweise sichtbar machen kann, was in menschlichen Gehirnen abläuft, wenn gelernt wird.

Im Rahmen institutionalisierter Bedingungen des Lernens bestehen durchaus Spielräume für Veränderungen (1). Dabei lassen sich Lernszenarien ausgestalten, die durch Nah- und Fernkommunikation gekennzeichnet sind (2). Ausgangspunkt für den Wunsch nach Veränderungen ist ein diffuses Unbehagen am Lernbetrieb, das zumeist beschwichtigt wird (3), jedoch einen Anknüpfungspunkt für Erkenntnisse der Neurobiologie bietet (4). Ein vergleichender Blick auf „,vorauseilende Pädagogik und Didaktik“ im Bereich der Schulen, die sich erst in den letzten Jahren der Neurobiologie geöffnet haben, kann zudem zeigen, welche Möglichkeiten sich für die Hochschulen bieten, den pädagogisch-didaktischen Vorsprung der Schulen durch ,nachholende Pädagogik und Didaktik mit Hilfe der Neurobiologie“" wett zu machen (5). Auf dieser Grundlage wird die Gliederung dieser Untersuchung erläutert (6).

\section{Lernen und seine Institutionalisierung}

Von der Tierwelt unterscheiden sich menschliche Gesellschaften dadurch, dass sie die ihnen gestellten Probleme zu analysieren und organisatorisch in unter- 
schiedlicher Weise zu bearbeiten suchen. ${ }^{1}$ Gelingt die Institutionalisierung, so werden Ämter eingerichtet und durch Personen besetzt, von denen erwartet werden darf, dass sie die ihnen übertragenen Aufgaben meistern. ${ }^{2}$ Beispielsweise werden im politischen Bereich Regierungen, Parlamente und Gerichte geschaffen, die das Zusammenleben im Staat durch geeignete Interventionen innen- und außenpolitisch absichern. Wie auch in anderen gesellschaftlichen Bereichen ist die geschaffene und reglementierte institutionelle Ordnung solange funktionsfähig, wie sie von den einbezogenen Mitgliedern einer Gesellschaft in der Grundtendenz mehrheitlich unterstützt wird. Erfüllt werden die gestellten Aufgaben mit Hilfe von rituell-symbolischen, vor allem sprachlichen und schriftlichen Mitteln. Die eingesetzten Amtsträgerinnen und Amtsträger machen den Bürgerinnen und Bürgern klar, dass und in welcher Weise die gemeinsamen Ziele erreicht werden können.

Was die hier interessierenden Hochschulen betrifft, so wird durch sie ein gesellschaftlicher Teilbereich institutionell ausgeformt, der dazu dient, dass Studierende - in der Fortführung schulischen Lernens - einerseits ihre Persönlichkeit ausbilden und fortentwickeln können. Andererseits wird ihnen die Möglichkeit gegeben, sich Wissensstoff anzueignen, vorhandene Wissensbestände zu erweitern und sich - vor allem gemäß den jüngsten Ansprüchen der Hochschulen verwertbare Kenntnisse anzueignen. In Zeiten der Digitalisierung, auf die im V. Kapitel (V.3.) noch speziell eingegangen wird, gewinnt diese Art des autonomen Lernens möglicherweise eine gesteigerte Bedeutung. Ihm steht kommunikatives Lernen gegenüber. Diejenigen, die das Lernen anleiten, können sich in einem engen Kontakt zu den Lernenden In neurobiologischer Perspektive verdient diese nahkommunikative Form des Lernens, wie sich zeigen wird, besonderes Augenmerk. Von ihr muss das Lernen in Fernkommunikation unterschieden werden, das vor allem im traditionellen Vorlesungsbetrieb Das $\square$ Lernergebnis, das dabei erzielt wird, bedarf in der Abgrenzung gegenüber dem Ertrag des Lernens in Nahkommunikation besonders sorgfältiger neurobiologischer Überprüfung.

Das an Hochschulen institutionalisierte Lernen wird - nach der Terminologie des amerikanischen Sozialphilosophen John R. Searle - von einer „kol-

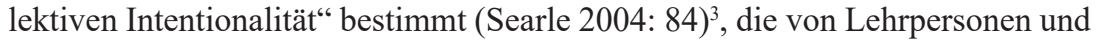

1 George Orwell wählt die „untierische“ Institution einer als Märchen konzipierten „Animal Farm“ (1945), um den Menschen seine Kritik an unmenschlichen und diktatorischen Einrichtungen nahezubringen.

2 In seiner erhellenden Studie Die Konstruktion der gesellschaftlichen Wirklichkeit (dt. Übers.: Reinbek 1997) verweist John R. Searle auf auszufüllende „Statusfunktionen“.

3 Der Evolutionsbiologe Michael Tomasello verwendet den Begriff der ,geteilten Inten- 
Lernenden gemeinsam getragen wird. Wie auch in anderen gesellschaftliche Bereichen gilt für Hochschulen, dass die kollektiv geteilte Position keineswegs mit den Wünschen aller Beteiligten übereinstimmen muss. ${ }^{4}$ Insoweit kann der Searle'schen „kollektiven Intentionalität“ eine, so könnte man formulieren, ,abweichende Intentionalität“" des Lernens an Hochschulen gegenübergestellt werden. Diese kann sich auf elementare Erkenntnisse der Neurobiologie stützen.

\section{Lernen in individueller Kommunikation mit Menschen und (Lern-)Materialien - Nahkommunikation und Fernkommunikation}

Alles Lernen ist in dem Sinne individuelles Lernen, dass aus der Umwelt kommende Impulse über die Sinne des Individuums im neuronalen Netzwerk verarbeitet werden, das in seinem Gehirn angelegt ist. ${ }^{5}$ Im Blick auf die Hochschulen lassen sich zwei Arten von Lernanstößen unterscheiden. Sie kommen einerseits von Menschen, die professionell das Lernen von Studierenden in bestimmten Lernszenarios - beispielsweise im Szenario der Vorlesung - anleiten. Andererseits kommen diese Anstöße von ausgewählten (Lern-)Materialien - beispielsweise Büchern -, die in autonomer Lernarbeit genutzt werden können.

Soweit die Professionals in ihren Lernszenarios solche Materialien selbst einsetzen, hat die Unterscheidung zwischen menschlich und material vermittelten Lernakten nur analytische Bedeutung. Sie wird dort real, wo es im Lernprozess breite Spielräume für autonome Hausarbeit gibt, die unabhängig von professionell angeleiteten Lernszenarios oder aber in deren Vor- oder Nachbereitung geleistet wird.

Wie sich in neurobiologischer Perspektive zeigen wird, kommt es für den Lernertrag darauf an, ob die Lernbeziehung eher auf Nahkommunikation oder auf Fernkommunikation angelegt ist. Im ersten Fall haben diejenigen, die das Lernen anleiten, engen Kontakt zu den Lernenden. Das gilt für Seminar-Veranstaltungen unterschiedlicher Ausgestaltung. Im zweiten Fall sind die Lernpartnerinnen und Lernpartner - zum Beispiel im Hörsaal - weit voneinander entfernt.

tionalität“, für ihn die Voraussetzung kooperativen menschlichen Handelns, von dem das Überleben abhängt (Tomasello 2010: 49ff.).

4 Vgl. zu diesem Auseinanderklaffen zwischen dem Handlungsgrund der Institutionalisierung und den Wünschen ausgewählter Gesellschaftsmitglieder: Searle 2004: 82; 87f.

5 Im Laborversuch wurden die Areale, von denen die subjektive Erlebnisfähigkeit getragen wird, u. a. ermittelt von: Vogeley/Newen 2007: 59-84. 
Lernen mit Menschen ist im Blick auf den Vorlesungs- und Seminarbetrieb immer auch Lernen in der Nahkommunikation mit Mit-Lernenden. Dieser Bezug zu den Mit-Lernenden, wird in der folgenden Analyse nur teilweise hergestellt, aber immer „mitgedacht“. Werden die je individuell Lernenden in ihrem Verhältnis zu denen, die das Lernen anleiten, emotional gepackt, so überträgt sich das lernfördernd auf die Interaktion, in der sich die miteinander Lernenden Dasselbe gilt umgekehrt bei unbefriedigenden emotionalen Schubkräften: Für je individuell Lernende führt das dazu, dass sich auch im „Lernkollektiv“ nur wenig lernfördernde Wirkungen ergeben.

\section{Diffuses Unbehagen an obrigkeitlicher Lehre und seine Beschwichtigung}

Jenseits von mangelhaftem organisatorischen Aufbau des Studiums, von Problemen mit unzulänglicher Raumsituation und fehlenden Möglichkeiten der Ausleihe von Büchern waren die Studierenden schon in den 1960er Jahren, der Studienzeit des Autors, mit ihrer Lernsituation ziemlich unzufrieden: Warum liefen die Seminare und Übungen so trocken und wenig inspirierend ab? Warum wurden die meisten Vorlesungen als so erlebt - und waren es offensichtlich auch? In der Assistentenzeit und danach, als der Autor vor allem Studierende der Politikwissenschaft in das Fach einführte und sie im Grundstudium betreute, wurde dieses diffuse studentische Unbehagen immer wieder bestätigt. Es wurde sowohl Ende der 1980er Jahre und dann wiederum in den Jahren 2010ff. (nach der Einführung von Bachelor- und Masterstudiengängen) erneut bekräftigt, als aussagebereite Studierende über mehrere Jahre hinweg im lockeren Gespräch und in Intensiv-Interviews offene Fragen zu ihrer Lernsituation beantworteten. ${ }^{6}$ Dabei ergab sich als später noch zu

Die wichtigste Grundlage meiner Studie ist „,episodische Empirie“ (=punktuelle Erlebnisse mit Studierenden).

Sie stammt aus meiner Studien- und Ausbildungszeit, die an der Universität Tübingen (1960/61) begann und sich dann am Otto-Suhr-Institut der FU Berlin (1961-1968) fortsetzte. Dort war ich nach dem Examen (Dipl.-Pol./1965) wissenschaftlicher Tutor und Doktorand, stand in der zweiten Hälfte der 1960er Jahre unter dem Einfluss der Studentenbewegung. Hinzu kommen Erfahrungen, die ich am Seminar für Politikwissenschaft an der Universität Bonn (1974-2007) und bei Aufenthalten an ausländischen Universitäten (Frankreich/Großbritannien/USA) machte.

Die ,systematische Empirie“ der Studie beruht hauptsächlich auf folgenden Grundlagen: Im Herbst 1988 und im Herbst 2016: Offene General-Interviews mit jeweils 20 Studierenden (Studierende unterschiedlicher sozial- und sprachwissenschaftlicher Fächerkombinationen und unterschiedlicher sozialer Herkunft); punktuelle Befragungen von 
erläuterndes wichtiges Ergebnis dieser - in die Breite und Tiefe gehenden qualitativen Befragungen, dass die Äußerungen des Unbehagens stets mit dem Bemühen verbunden waren, ihren kritischen Unterton herabzuspielen und die Lernabläufe positiver darzustellen, als sie erlebt wurden. Typisch ist beispielsweise der nachgezogene Kommentar: „Aber der Herr X gibt sich ja so viel Mühe.“

Soweit es hartnäckiger Befragung gelang, diesen Hang zur Beschwichtigung zu unterlaufen, so verstärkte sich der Eindruck, dass Vieles an der Lernsituation tatsächlich ,ärgerlich“ war. (Es wurde am Anfang der Interviews gefragt: „Was ärgert dich an der Universität"?) Sonst hätte es keine Veranlassung gegeben, die gegebenen Antworten aufzupolieren.

Nun gibt es seit den 1970er Jahren eine sich auch international durchsetzende Tendenz, den Studierenden Fragebögen vorzulegen und sie darum zu bitten, einzelne Lehrveranstaltungen zu bewerten. Im Ganzen ergeben sich dabei - in Übereinstimmung mit dem Ergebnis meiner qualitativen Befragungen - auffällig positive Beurteilungen. Noch wichtiger ist, dass eine sorgfältige Betrachtung belegt, dass die Befragten beträchtliche Schwierigkeiten haben, ihre Lernsituation einzuschätzen.

Dies zeigt sich auch bei der Auswertung der Befragungsergebnisse. Insbesondere in den USA haben Privathochschulen ein Interesse daran, die „Lehrqualität" ihres Personals zu ermitteln, von der ihr Ranking maßgeblich abhängen kann. Wie effektiv ist die sogenannte Lehre? Neben der Beurteilung der Lehrpersonen geht es immer auch um eine Bewertung der Lernformate. Welche Schlussfolgerungen aus den teilweise widersprüchlichen Daten zu ziehen sind, wird jedoch kaum Zu den Ausnahmen gehört ein neueres Untersuchungsprojekt, das sich auf amerikanische Hochschulen bezieht (Stroebe 2016/2017). Die aus ihm abgeleiteten Zweifel an der Aussagekraft der erhobenen Daten lassen sich unschwer auf europäische Verhältnisse übertragen.

Studierenden naturwissenschaftlicher Fächer. Ausgangsfrage: „Was ärgert mich an der Universität?“; Zusatzfragen: Im Gespräch aufkommend, assoziativ entwickelt und ins Detail gehend.

Repräsentative Meinungsbefragungen des Bundesministeriums für Bildung und Wissenschaft/Forschung (Studiensituation/Studienerfahrungen und Studienorientierungen - Konstanzer Forschungsteam um Tino Bargel u. a. ab 1982/83).

Publikationen und Dokumentationen zur Hochschuldidaktik, die durch die Studentenbewegung angeregt wurden. (Es sind diese Texte aus den 1960er bis 1980er Jahren, die bis heute - ergänzt um neuere Texte zum ,Shift from teaching to learning“ (2007ff.) den hochschuldidaktischen Grundstock liefern.)

Publikationen aus der Wissenschaftsgeschichte, der Bildungs- und Wissenschafts-Soziologie, der Erziehungswissenschaft und der Sozialisationsforschung. 
Der Autor Stroebe zitiert die Schlussfolgerungen eines Berichts, der sich auf eine „Lehrveranstaltungsevaluation“ bezieht, die im Jahre 2001 an der Universität Stuttgart durchgeführt wurde: „In Anbetracht der beschränkten Aussagekraft von Lehrveranstaltungsevaluationen durch Studierendenbefragung an der Universität Stuttgart ist große Vorsicht bei der Verwendung der Evaluationsergebnisse geboten. Das primäre Ziel soll und kann nur die individuelle Verbesserung der evaluierten Lehrveranstaltung durch den jeweiligen Dozenten auf Basis des Feedbacks der Studierenden sein." (Stroebe 2017: 137).

Stroebes Zustimmung zu dieser Stuttgarter Evaluationsbilanz beruht darauf, dass sich bei der Interpretation der Befragungsdaten vor allem zwei Schwierigkeiten ergeben. Zum einen glaubt er einer großen Zahl der befragten Studierenden mit guten Gründen unterstellen zu können, dass für sie der Unterhaltungswert einer Veranstaltung wichtiger ist als die Kenntnisse, die vermittelt werden. Zum andern argumentiert er, dass das Urteil über die Lehrqualität dadurch positiv oder negativ werden kann, dass eher hohe oder aber eher niedrige Anforderungen - was ist wünschenswert? - gestellt werden (Stroebe 2017: 136).

Hat Stroebe recht, so steht die Antwort-Kompetenz der Befragten in Frage. Die Zweifel an der Aussagekraft der Daten verweisen auf Unsicherheiten, die hinter jenem wohlwollend beschwichtigten Unbehagen stehen, das aus eigenen Beobachtungen und Befragungen hervorgeht. Woher sollen Studierende ein Verständnis davon haben, worauf es beim Lernen ankommt? Es wird nur allzu deutlich, dass es ihnen an klaren Kriterien fehlt, über die sie verfügen müssten, um die Qualität des Lernens präzise bewerten zu können.

Zweifel am Urteilsvermögen der Studierenden müssen auch geltend gemacht werden, wenn Lehrpersonen, die von obrigkeitlicher Lehre abzuweichen versuchen, sehr oft auf Ablehnung stoßen. Das gilt insbesondere für die Erfahrungen mit dem im V. Kapitel behandelten sogenannten Präsenz-Lernen, das von den Studierenden oft nur zögerlich oder auch gar nicht angenommen wird (vgl. Loviscach 2016). Wie kompetent sind nun aber diejenigen, die Lernprozesse anleiten? Können sie die Qualität ihrer Lehre einschätzen?

Lassen sie sich überhaupt zu Antworten auf präzise Fragen zu ihrer „Lehr-Leistung“ herausfordern, so zeigen sich große Unsicherheiten. Immer noch gültig und repräsentativ scheint das ehrliche Eingeständnis des Politologen Wilhelm Hennis vom Ende der 1960er Jahre: Er sei im Zweifel darüber, ob Lernstoffe autoritativ von oben „eingetrichtert“ werden müssten oder es selbstbestimmter Initiativen von unten bedürfe (Hennis 1969).

Was speziell die Vorlesungs-Leistung angeht, die nach wie vor im Mittelpunkt von Lehrdeputaten steht, so wurde der leitende Redakteur der Fachzeitschrift des deutschen Hochschulverbands „Forschung \& Lehre“ 2012 mit dem Vorschlag des Autors konfrontiert, einen Aufsatz zur Kritik des Vorlesungs- 
betriebs zu publizieren (in: Forschung \& Lehre 4/2012). Seine erste Reaktion war: „Warum denn? Es funktioniert doch.“ Sie steht für einen der Tradition deutscher Hochschulen entsprechenden Mainstream, der in derselben „Forschung \& Lehre" im Sommer 2017 durch den Text der Hamburger Professorin Christiane Bender repräsentiert wurde. In einer Kurzfassung publiziert („Forschung \& Lehre, 8/2017), deckt sich die Auffassung der Autorin mit dem traditionellen Vorlesungslob, das in im IV. Kapitel noch näher illustriert wird. Eine Universität ohne Vorlesungen erklärt Bender für ,,möglich, aber sinnlos“. Ein besonderes Augenmerk richtet die Autorin auf Elite-Studiengänge, in denen Vorlesungen einen „festen Platz" haben sollten. (Die längere Fassung liegt dem Verfasser in einem Manuskript von 2016 vor.)

Solches Festhalten am Lernformat Vorlesung und auch daran, dass im Seminar- und Übungsbetrieb der Hochschulen Studierende als Vorlesende ihre Referate vortragen und auf diese Weise das Vorlesungsprinzip variiert wird, steht im Widerspruch zur folgenden Beobachtung: Wenn Kolleginnen und Kollegen „unter sich reden“, so machen sie in der Regel kein Hehl daraus, dass sie, speziell was die Vorlesung betrifft, daran zweifeln, ob die zeitintensive Vorbereitung und die anstrengende Umsetzung in einem angemessenen Verhältnis zum Ergebnis stehen. Wer solche Gespräche kennt, weiß aber auch, dass dieses Votum zumeist durch abwiegelnde Bemerkungen wird: Wenn man am Anfang des Semesters darüber stöhnt, dass es ,nun wieder losgeht", so wird doch zugleich betont, dass man ,es doch irgendwie hinkriegt". Nicht mit von der Partie sind einige wenige rhetorische Ausnahmetalente, bei denen es am Unbehagen und folglich auch am begleitenden Stöhnen fehlt.

Das widersprüchliche Festhalten am Vorlesungsprinzip, das für den Mainstream kennzeichnend ist, löst sich auf, wenn man sich klar macht, dass die Bagatellisierung des eingestandenen Unbehagens an der Vorlesungsleistung als Beschwichtigung verstanden werden kann. Die Parallele zur ambivalenten Einschätzung der Lernsituation durch Studierende ist deutlich: Auf beiden Seiten des „Vorlesungspults“ wird das diffuse Unbehagen an der routinemäßig etablierten Praxis dadurch akzeptabel, dass es abgewiegelt wird.

\section{Die Herausforderungen der Neurobiologie}

Was die gesellschaftlichen Rahmenbedingungen angeht, so muss nicht im Einzelnen erörtert werden, dass die vielfältiger Medien, gestützt durch digitale Kommunikation, zu einer riesenhaften Ausweitung des potentiellen Lernstoffs geführt hat. Im wahrsten Sinne des Wortes „,vergeht den Lernenden Hören und Sehen“. In ihrem unruhig vibrierenden Hirn, wie es von der Kamera erfasst wird, steckt eine unbezähmbare Neugier, die je- 
doch - aufgrund beschränkter Ressourcen - den von außen kommenden und im Innern zu verarbeitenden Herausforderungen nur zum kleinen Teil genügen kann. Umso problematischer ist es deshalb für die Lernenden geworden, sich speziellen Lern- und Ausbildungsinhalten zu öffnen und zielgerichtete Lernbemühungen vor ablenkenden

$\mathrm{zu}$ schützen. Das bedeutet keineswegs, dass beispielsweise Verbindungsstudenten in zurückliegenden, weniger herausfordernden Zeiten nicht die Möglichkeit gehabt und genutzt hätten, die Angebote an Geselligkeit wichtiger zu nehmen als ihr Studium. Es bedeutet jedoch, dass diese sich zu Lern- und Examenszwecken wenigstens für kurze Phasen in Klausur begeben konnten. Da eben dies unter der aktuellen medialen Dauerberieselung und digitalen Daueraktivität nur unter großen Schwierigkeiten möglich erscheint, ist die Neurobiologie in besonderer Weise gefragt. Es ist darüber nachzudenken, ob elementare Kenntnisse der Abläufe im lernenden Gehirn zumindest dabei helfen können, etablierte Lernformate auf ihren Lernertrag hin zu überprüfen. Darüber hinaus stellt sich die Frage, ob sich mit Hilfe der Neurobiologie Lernformate bestimmen lassen, die sowohl den besonderen Zeitumständen als auch dem neuronalen Lernapparat angemessen sind.

Wenn Studierende speziell gefragt werden, warum sie auch dann zu Vorlesungen gehen, wenn diese nicht sind, so laufen ihre Antworten zumeist auf die folgende Erwartung hinaus: Die Lehrenden beherrschen den Wissensstoff ihres Faches und seiner Teilgebiete. In einem unbekannten Feld erspart es ihnen das eigene Studium, wenn in der Vorlesung die wichtigen Fakten in zusammenfassender und systematisierter Form dargeboten und darüber hinaus Anregungen zur Nacharbeit und zur Vorbereitung auf die Klausuren gegeben werden. Dieser Erwartung versuchen die Vorlesenden zu entsprechen.

Dabei tut man der großen Mehrheit auf beiden Seiten des Vorlesungspults nicht unrecht, wenn ihr unterstellt wird, dass sie vom frontalistischen Lernformat - parallel auch zum Frontalismus in Seminaren und Übungen - stillschweigend das Folgende erwartet: Das Wissen der Lehrenden, wodurch es inhaltlich auch bestimmt sein mag, wird eins zu eins auf die Lernenden übertragen. Dem liegt ein objektivistisch-empiristisches Modell von Lernen zugrunde, wie es schon von den englischen Philosophen Locke, Hume und Berkeley vertreten wurde. Danach gleicht das menschliche Gehirn einer Wachs- oder Schiefertafel - auch einem leeren Blatt (Pinker 2003: bes. Teil I) -, auf der die über die Sinne eingehenden Informationen aufgezeichnet werden (Dehaene 2009: 14f.). Unberücksichtigt bleibt eine dem Gehirn inhärente Struktur, ein Netzwerk, in dem alle einlaufenden Informationen in ebenso eigenständiger wie eigensinniger Weise verarbeitet werden. Soweit Minderheiten unter den Studierenden und den Lehrpersonen aufgrund ihrer Intuition oder dank einer sorgfältigen Auswertung pädagogisch-didaktischer Erfahrungen gegen frontale Lehre aufbegehren, fehlt es auch hier an elementaren neurobiologischen Kenntnissen. 
Es ist unübersehbar, dass Schulen und Hochschulen von der Neurobiologie in unterschiedlicher Weise herausgefordert werden. Der Schulbetrieb ist schon immer auf alltägliche Nahkommunikation ausgerichtet. Sie bedarf - jenseits aller neurobiologischen Kenntnisse - pädagogisch-didaktischer Ausgestaltung, wie immer diese bestimmt sein mag. Demgegenüber ist der Hochschulbetrieb, nicht erst seit es die Massenuniversität gibt, dadurch gekennzeichnet, dass diejenigen, die das Lernen anleiten, und diejenigen, die lernen wollen, traditionell relativ weit voneinander entfernt sind. Das Prinzip frontalistischer Lehre kommt fast selbstverständlich zur Anwendung. Bei der Frage, ob und wie sich der Einblick ins Gehirn der Lernenden für die Pädagogik und Didaktik nutzen lässt, sich daher Schulen und Hochschulen in einer unterschiedlichen Ausgangslage.

\section{Lernen in Nahkommunikation an Schulen: Von intuitiver zu neurobiologisch informierter Pädagogik und Didaktik}

In der Sozialforschung hat sich immer wieder gezeigt, dass Vertreterinnen und Vertreter traditioneller Methoden und Techniken den Mehrwert neuer Zugriffe anzweifeln. Insbesondere wird in Frage gestellt, ob die empirische Verbreiterung der analytischen Basis - zumal durch statistische Daten - zu neuen Erkenntnissen führt. Dies gilt auch für die zögerliche Öffnung der Schuldidaktik gegenüber der Neurobiologie. Im Folgenden kommen wir noch auf den Bremer Neurobiologen Gerhard Roth zurück, der bislang als Einziger den Versuch gemacht hat, die Schuldidaktik neurobiologisch zu fundieren und Vorschläge für die Unterrichtspraxis zu machen (Roth 2011; vgl. bes. Kap. 12). Entsprechend hat sich die Hochschuldidaktik gar nicht oder doch nur sporadisch auf die Neurobiologie eingelassen (Böss-Ostendorf/Senft 2018; 2016; vgl. Exkurs 3). Doch blicken wir zunächst auf die Schuldidaktik.

Eine einleuchtende Begründung für solche Reaktionen könnte in der folgenden Überlegung liegen: Wir Didaktikerinnen und Didaktiker achten bei der Anwendung unserer Konzepte sorgfältig darauf und überprüfen auch durch Begleitforschung, was bei den Schülerinnen und Schülern ankommt und an ihnen vorbeigeht. Indem wir so vorgehen und bislang so gearbeitet haben, ging es immer darum, uns verborgene Abläufe in ihrem Gehirn vorauszusetzen und - auch ohne Neurobiologie - stillschweigend zu berücksichtigen, was ihr neuronaler Lernapparat aufnehmen und was er nicht fassen kann. 


\section{Von Pestalozzi/Herbart zu Spranger/Litt u. a.}

Das Nachdenken über Prinzipien des Lernens führte im Übergang vom 18. zum 19. Jahrhundert zu einer schulbezogenen Pädagogik, die sich sowohl auf die Lerninhalte als auch in didaktischer Intention auf die Lernwege bezog, auf denen diese den Schülerinnen und Schülern vermittelt werden sollten. In der Praxis erprobte Lehrpersonen, die sich intuitiv zu pädagogisch-didaktischen

$\mathrm{Zu}$ ihnen gehörte der Schweizer Querdenker Johann Heinrich Pestalozzi, der 1807 Nachforschungen über den Gang der Natur in der Entwicklung des Menschengeschlechts publizierte (Löwisch 2002). Er stützte sich dabei auf ein ihm überliefertes und zuletzt von Rousseau geprägtes Verständnis der menschlichen Natur, dem es an jeglicher Kenntnis der Abläufe im Gehirn des Menschen fehlte. Dasselbe galt für den zunächst als Hauslehrer in Bremen arbeitenden Johann Friedrich Herbart. Wie Pestalozzi war er philosophisch gebildet und hoch ambitioniert. (Herbart übernahm 1808 den ehemaligen Lehrstuhl Kants in Königsberg.) Beide bemühten sich um die Begründung von hier nicht im Einzelnen interessierenden Lerninhalten, um die Erziehung zu Moralität und zu ästhetischem Urteilsvermögen (Matthes/Heinze 2003: 114f.). Als sozial engagierten Männern (Pestalozzi kümmerte sich um Erziehungseinrichtungen für Arme, Kriegswaisen und „Straßenkinder“; Löwisch 2002: 154f.) war es ihnen insbesondere darum zu tun, den Unterricht auf die Bedürfnisse der Lernenden abzustimmen.

Dieser Weichenstellung folgend, wurde dann die praxisnahe Tradition im 19. Jahrhundert, vor allem in dessen zweiter Hälfte und ins 20. Jahrhundert hineinreichend, fortgeführt. Große Namen wie Eduard Spranger und Theodor Litt übernahmen die Grundintentionen von Pestalozzi und Herbart. Vor allem Andreas Flitner, Erich Weniger und Wolfgang Klafki eiferten im 20. Jahrhundert ihren Vorgängern nach. Auf der Grundlage eigener praktischer Erfahrungen und von diesen abgeleiteten Intuitionen versuchten sie fortzusetzen und fortzuentwickeln, was ihnen unter neuen Bedingungen geboten erschien (Klafki 1963).

Unter dem der Studentenbewegung und auf dem Hintergrund der NS-Geschichte kam es in den 1970er Jahren dazu, dass sich die schulbezogene pädagogisch-didaktische Diskussion auf Fragen politischer Bildung verengte. Dabei ist wiederum auffällig, dass es vor allem Praktiker waren, die diesen Schwerpunkt setzten und sich - bis zur Gegenwart auch in den Bereich der Weiterbildung hineinwirkend (Handbuch 2016) - darum bemühten, den Unterricht auf das Interesse der Schülerinnen und Schüler zuzuschneiden (Schmiederer 1977; bes. auch: Fischer 1975/77). 


\section{John Hatties Inventur neuerer (internationaler) Schulforschung}

Der australische Schuldidaktiker und -theoretiker John Hattie erregte sowohl beim Fachpublikum als auch in einer breiteren Öffentlichkeit großes Aufsehen, als er 2013 das Ergebnis langjähriger Recherchen zur Schulforschung publizierte. Sein Beitrag bestand darin, dass er ca. 80.000 Studien auswertete und interpretierte, die er vom Ende der 1970er Jahre bis 2008 in der internationalen Schulliteratur machen konnte. In seinen über 800 Metaanalysen ging es ihm darum, aus der in den Studien enthaltenen Empirie die Faktoren abzuleiten, die in unterschiedlicher - mit Methoden der Statistik zu ermittelnder - Stärke auf den Ertrag schulischen Lernens einwirken (Hattie 2013; Hattie 2014).

Dabei kommt er zu einem Ergebnis, das auf Folgendes hinausläuft: Gelingendes Lernen wird maßgeblich von den Lehrpersonen bestimmt. Es ist davon abhängig, dass sie „,das Lernen durch die Brille der Schülerinnen und Schüler sehen“ (Hattie 2014: 280) und daher in der Lage sind, auf den wechselnden Lernstand der Lernenden zu reagieren. Dieses Feedback (Hatties Begriff) der Lehrpersonen erfordert umgekehrt Reaktionen der Lernenden, aus denen sich ergibt, was sie verstanden und was sie noch nicht verstanden haben.

Was speziell die Lehrpersonen angeht, so sieht sie Hattie in der Rolle von Regisseuren, denen es obliegt, ,,die Lernenden zu verändern, sie immer wieder herauszufordern und an ihre Grenzen zu bringen“. Dagegen ist ihm die bloße Rolle eines Moderators (oder auch Begleiters) nicht direktiv genug, wenngleich er den Lernenden Spielräume zu „entdeckendem Lernen“ geben will (Interview, in: Die Zeit, 2013/Nr. 19). ${ }^{7}$

7 Hatties Begriff des Regisseurs (auch: des Guides) akzentuiert einseitig die für den Schulbereich durchaus angemessene direktive Aufgabe der Lehrpersonen. Der Begriff lässt jedoch zu wenig Spielraum für die auch von Hattie geforderte Mitwirkung der Schülerinnen und Schüler. Im Rahmen des hier auf Hochschulen bezogenen Konzepts dialogischen Lernens von Erwachsenen zielt die etwas umständliche Formulierung des „Anleitens des Lernens“ auf die tendenzielle Offenheit der Lernbeziehungen zwischen Lehrpersonen und Studierenden, in denen die variable Mitwirkung beider Dialog-Partnerinnen und -Partner gefragt ist. Diese wird auch durch den zumeist direktiv verstandenen Begriff des „Coachings“ verfehlt, der deshalb genauso vermieden wird wie der Regisseur-Begriff.

Streng genommen müsste der von Hattie übernommene Begriff der Lehrpersonen durchgängig in Anführungszeichen gesetzt werden, um zu signalisieren, dass diese nicht nur direktive Aufgaben haben. 
Zum Vergleich der intuitiv fundierten Inventur John Hatties mit der neurobiologisch informierten Schulforschung von Gerhard Roth

Was den schon erwähnten pädagogisch-didaktischen Zugriff des Neurobiologen Gerhard Roth angeht, der vor Hatties Publikation liegt, so überschneidet er sich mit der Darstellung allgemeiner und spezieller Voraussetzungen des Lernens an Hochschulen, die hier im II. Kapitel gegeben wird.

Auffällig ist, dass Roths pädagogisch-didaktische Anwendung, die hier nicht im Einzelnen wiedergegeben werden kann, sich im Kern mit Hatties Schlussfolgerungen deckt. In einer salopp-metaphorischen Zusammenfassung dessen, was auch schon Roths Titel zum Ausdruck bringt (Bildung braucht Persönlichkeit), sieht er Hatties Lehrpersonen - bei ihm sind es Persönlichkeiten - „mit den Lernenden einen kognitiv-emotionalen Tanz aufführen“ (Roth 2011: 312). Der Hauptunterschied zu Hatties erfahrungsbasierten Intuitionen ist, dass Roth die neuronalen Prozesse, die der Kognition - das heißt dem Bewusstsein - sowohl der Lehrpersonen als auch der Lernenden unzugänglich sind, in großer Differenziertheit analysiert. Von ihnen wird ertragreiches Lernen getragen. Beteiligt sind unter anderem und vor allem die für Emotionen zuständigen neuronalen Areale. Diese wiederum sind mit den Abschnitten des Netzwerks verknüpft, die es sowohl Lehrpersonen als auch Lernenden ermöglichen „durch die Brille“ des je anderen zu schauen. Dabei handelt sich um Abschnitte, die für empathische Fähigkeiten zuständig sind (vgl. Kap. II., 2.). Unter Mitwirkung von Botenstoffen sorgen sie für die enge Bindung zwischen den Lernpartnerinnen und -partnern (Roth 2011: 56f.; 292f.), die von beiden Autoren als unverzichtbare Voraussetzung für ertragreiches Lernen erkannt wird. Demnach ist dieses nach der hier eingeführten Terminologie sowohl für Hattie als auch für Roth von einer intensiven Ausgestaltung der Nahkommunikation abhängig.

Ist nun aber in der Perspektive Hatties und in der Sicht der pädagogisch-didaktischen Bemühungen, auf denen er aufbaut, Roths Neurobiologie überhaupt gefragt? Die schon angedeutete erste Antwort verweist darauf, dass das von Hattie entworfene Lernszenario nicht nur präzisiert und ausdifferenziert, sondern zugleich gegenüber jenen Positionen bekräftigt und gestärkt wird, die ertragreiche Lernprozesse im Rahmen von Fernkommunikation für möglich halten. Wichtiger ist eine zweite Antwort, die sich auf die hier interessierenden Hochschulen bezieht. Ihnen kann die Neurobiologie aus einer Tradition obrigkeitlicher Lehre heraushelfen. 


\section{Neurobiologisch informiertes Lernen an Hochschulen gegen obrigkeitliche Lehre - zur Gliederung}

Im I. Kapitel wird die Tradition obrigkeitlicher Lehre skizziert, die mit pädagogisch-didaktischer Indifferenz verbunden ist. Im II. Kapitel werden einige unverzichtbare Grundkenntnisse über den neuronalen Lernapparat vermittelt (II.1). Es wird geklärt, welches in ihm liegende Potential ausgeschöpft werden muss, damit es zu ertragreichem Lernen kommen kann (II.2). Zu begründen ist, warum Lernprozesse als Macht-Diskurse verstanden werden müssen (II.3.). Darauf aufbauend lässt sich aus neurobiologischer Sicht belegen, dass sowohl die Ausgestaltung der allgemeinen Lern-Konstellation (III. Kapitel) als auch die vorlesungsorientierten Lernformate (IV. Kapitel) nicht den Anforderungen ertragreichen Lernens genügen, die der menschliche Lernapparat stellt.

Speziell zeigt sich im III. Kapitel, wie ungebrochen obrigkeitliche Lehre auch noch nach der Bologna-Reform (Wagner 2010: 7. Kap.) - in die Gegenwart hineinreicht. Beschrieben wird, dass es die Lernenden im Macht-Diskurs mit Ritualen, mit Notengläubigkeit und mit herablassender Indifferenz zu tun haben (III.1.), die sie in die Winzigkeit drängen. In neurobiologischer Perspektive wird erkennbar, dass aus dieser Diskurslage die Bereitschaft der Lernenden rührt, sich in ihrer großen Mehrheit ruhigstellen zu lassen und sich gegenüber hochschuldidaktischen

sowie mangelnder Betreuung und Beratung nachsichtig zu verhalten (III.2.).

Im IV. Kapitel, soweit es sich speziell auf den Vorlesungsbetrieb und dessen Bewertung bezieht, wird klar, dass dieser in die allgemeine Lern-Konstellation der studentischen Unterordnung passt (IV. 1./2.). Der differenzierte Bezug zu den neurobiologischen Grundlagen ertragreichen Lernens macht klar, dass der frontalistische Ansatz der Massenvorlesungen diese Grundlagen ignoriert (IV.3.).

Im V. Kapitel werden hochschuldidaktische Anregungen aufgenommen, die geeignet sind, der Tradition obrigkeitlicher Lehre Paroli zu bieten. Da es darum geht, diesen intuitiv-erfahrungsbasierten Vorschlägen eine neurobiologische Grundlage zu geben, werden die schon im II. Kapitel vermittelten neurobiologischen Grundinformationen vertieft. Gezeigt wird, was beim Lernen auf der synaptischen Ebene des Gehirns geschieht (V.1.). Im Anschluss daran lassen sich die Grundzüge eines Konzepts dialogischen Lernens entwickeln (V.2.). Dem wird eine kursorische Darstellung der in Zeiten der Digitalisierung verfügbaren Möglichkeiten individuellen Lernens hinzugefügt (V.3). Darüber hinaus wird überlegt, wie sich - im Rahmen des Konzepts dialogischen Lernens - traditionelle Lernformate mit digitalisierten Formaten kombinieren lassen (V.4.). Daraus ergeben sich veränderte Anforderungen an diejenigen, die 
das Lernen anleiten (V.5.). Schließlich kann prinzipiell abgeschätzt werden, welche Chancen sich Hochschulen bieten, wenn sie dazu bereit sind, sich am Konzept dialogischen Lernens zu orientieren. (V.6.).

Den für die Empirie der untersuchten Lernprozesse zentralen Kapiteln III. und IV. wird jeweils eine Zusammenfassung angefügt. Die wichtigsten Ergebnisse der Studie werden in einer Schlussbilanz verdichtet. 


\section{Der Kontext des Lernens in Geschichte und Gegenwart}

\section{Zur pädagogisch-didaktischen Indifferenz im Obrigkeitsstaat}

Was hier als pädagogisch-didaktische Indifferenz beschrieben wird, geht auf Zusammenhänge des Obrigkeitsstaats zurück, in denen sich eine Tradition obrigkeitlichen Lehrens etablieren konnte. Obwohl sich die Bedingungen grundlegend geändert haben, lebt diese Tradition auch in der Gegenwart fort.

\section{Der Ansatz obrigkeitlicher Lehre und das Vorlesungsprinzip}

Bedenkt man, dass die Institutionalisierung des Forschens und Lernens in den Rahmen von Grafschaften, Herzogtümern, Königs- und Kaiserreichen eingefügt war, so schärft dies den Blick für den Zusammenhang zwischen den gesellschaftlich-politischen Rahmenbedingungen und einem obrigkeitlichen Ansatz der Lernbeziehungen. Parallel zu einem Verständnis des Regierens, nach dem sich die Regierten den Anordnungen der Regierenden zu unterwerfen haben, stehen die Lernenden der Hochschulen unter dem „Diktat“" von Lehrpersonen.

Versucht man, die Entwicklung der Hochschulen seit ihrer Gründung im Mittelalter über die Epoche der Reformation bis ins 18./19. Jahrhundert hinein zu überblicken, so beruhte der obrigkeitliche Ansatz zunächst - zumal in der zentralen theologischen Fakultät - auf einer lange behaupteten und nur langsam schwindenden Lehr-Autorität, die auf der Grundlage des kirchlichen Lehramts ausgeübt wurde. Speziell in der medizinischen Fakultät wurde über die Jahrhunderte hinweg gelernt, was die Ärzte - speziell die Chirurgen - kraft ihrer Zuständigkeit für Gesundheit und Überleben an die ihnen nachfolgenden Lernenden weitergaben. In der artistisch-philosophischen Fakultät ist es der mit tiefschürfenden Denkbemühungen verbundene Anspruch auf Lebensklugheit und Wahrheit, der - alle anderen Fächer übergreifend - bewundernde Unterordnung einfordert. Als sich die Naturwissenschaften in der zweiten Hälfte des 19. Jahrhunderts etablierten, gewannen ihre Hochschulvertreter dadurch Lehrautorität, dass von ihrem Forscher- und geist - unter Einschluss der sich gleichzeitig ins Szene setzenden Nationalökonomen das ökonomische und soziale Wohl der Gesellschaft abhängig zu sein schien.

Schaut man etwas genauer auf den obrigkeitlich geprägten Lernbetrieb der Hochschulen, so wurde das „Diktat von oben“ unter den technischen Bedingungen des Mittelalters in der Weise praktiziert, dass den Lernenden im Hörsaal Texte mündlich diktiert und erläutert wurden. Diese Praxis des Vorlesens 

Buchdrucks zunächst noch selten und die Bestände der Bibliotheken relativ klein waren (Ellwein 1997: 53).

Als dann aber nach und nach der Eindruck entstand, dass das Ablesen von Texten in einen „geistlosen Schulbetrieb“ abglitt (Straub 2007: 15), kam die Zeit ambitionierter Vorlesungen, die nicht nur zum Lehrbetrieb der Universitäten gehörten. Im 19. Jahrhundert richteten sie sich als „Arienabende“ und „Pelzmantelkollegs“" auch an eine breite bürgerliche Öffentlichkeit (Straub 2008: 25). Dabei wurde die Neigung, Vorlesungen zu halten, dadurch gestützt, dass die Professoren, die nur ein geringes staatliches Grundgehalt bezogen, materiell von den privaten Gebühren abhängig waren, die ihnen die Vorlesungstätigkeit einbrachte (Ringer 1983: 43).

Welch enger Zusammenhang zwischen dem Vorlesungsprinzip und den gesellschaftlich-politischen Rahmenbedingungen besteht, zeigte sich in den deutschen Ländern und speziell im übergewichtigen - speziell auch bildungspolitisch dominierenden - Preußen. Der deutsch-amerikanische Historiker Fritz K. Ringer beschreibt diese Bedingungen als System ,intellektueller Mandarine“, die zusammen mit Ärzten, Rechtsanwälten, Geistlichen und Staatsbeamten eine gesellschaftliche Elite bildeten (Ringer 1983: 12ff.). Der Begriff der Ordinarienuniversität passt zu den hierarchisch-organisatorischen und autoritär-mentalen Strukturen Preußen-Deutschlands und dazu, dass es seit dem Dreißigjährigen Krieg keine Bürgerkriege, keine erfolgreichen Oppositionsbewegungen und keinen religiös inspirierten politischen Nonkonformismus gegeben hatte (Mosse 1988: 281f.). Entsprechend war der Widerspruchsgeist schwach, eine Grundlage dafür, dass „vorlesende Mandarine“, unter ihnen Fichte, Schleiermacher und Hegel, sich an den Hochschulen eine unantastbare Spitzenstellung verschaffen konnten. Dazu passt, dass man zum Studium ,an eine bestimmte Universität (ging), um einen bestimmten Prof. zu hören ..."(Ellwein 1997: 132).

Wer Geschichte studierte, traf an der Berliner Friedrich-Wilhelms-Universität auf den berühmten Heinrich von Treitschke. Auf ihn bezieht sich eine Episode, die oft erzählt und zitiert wird, um das zeittypisch anmaßende Verhalten von Männern gegenüber diskriminierten Frauen zu illustrieren. Im hier interessierenden Zusammenhang illustriert die imperiale Pose, in der Treitschke auftrat, in prinzipieller Weise obrigkeitliches Lehren: Als Treitschke vom Vorlesungspult aus unter seinen Hörern eine Frau sitzen sah, stieg er vom Podest herab, ging auf die Frau zu, nahm sie an der Hand und führte sie unter dem Raunen der Anwesenden aus dem Saal hinaus. Der Wissende nimmt hier für sich in Anspruch, die nach ihrer Einschätzung Unwissenden auszuwählen, sie von oben herab an ihrem Wissen teilhaben zu lassen und von oben herab zu belehren. 


\section{Minderheitliches Unbehagen an obrigkeitlicher Lehre}

Wilhelm von Humboldt, dem preußischen Reformer, an dem sich die Diskussion über Lernprozesse an Hochschulen bis heute orientiert, ging es im Kern darum, „dass der junge Mann zwischen der Schule und dem Eintritt in das Leben eine Anzahl von Jahren ausschließlich dem wissenschaftlichen Nachdenken an einem Ort widme, der viele Lehrer und Lernende in sich vereinigt“ (zit. nach Straub 2007: 15). Angesichts eines solchen Konzepts wird klar, dass Humboldt mit den etablierten Vorlesungs-Diktaten, die zum Auswendiglernen führen sollten, sehr unzufrieden war. Als Rahmen, in dem sich die „Gemeinschaft zwischen Lehrenden und Lernenden“ herstellen ließ, bot sich das Seminar-Gespräch an (Straub 2008: 24f.). Am Rande des routinemäßigen Vorlesungsbetriebs wurde es, durchaus im Sinne Humboldts, vermutlich vielerorts eingeführt, beispielsweise durch den Historiker Georg Waitz, der diese Art des Lernens Mitte des 19. Jahrhunderts an der Universität Göttingen praktizierte (Ellwein 1997: 141).

Bezweifelt werden muss, dass die Vorlesenden, die nach Humboldts Vorstellungen ein forschungsorientiertes professorales Lehramt ausübten, in ihren Auftritten im Hörsaal - wie Humboldt meinte - eine reizvolle Möglichkeit sahen, die „Muße“ ihres eher mühevollen Studiums im „freie(n) mündliche(n) Vortrag" zu unterbrechen. Dies umso mehr - so sah es Humboldt -, da sie es mit Zuhörern zu tun hatten, „unter denen doch immer eine bedeutende Zahl selbst mitdenkender Köpfe ist" ${ }^{\text {" }}$. Eine solche Einschätzung zeigt, dass es in Humboldts Universität beim Thema Vorlesung um inhaltlich-gedanklichen Reichtum und nicht um die Frage ging, auf welche Weise Inhalte vermittelt werden konnten.

Auch vorher schon gab es einzelne Initiativen zur eher improvisierenden des Vorlesungsbetriebs, auf die Walter Jens bei seinem Gang durch die Tübinger Universitätsgeschichte aufmerksam wurde. Nicht zufällig fallen sie in die 1820er und 1830er Jahre, also ins Vorfeld der Revolution von 1848. Beteiligt waren sowohl Theologen als auch Juristen, die an die Stelle der systematischen Vorlesung die „Konversation“ setzen wollten. Über eine „Dialogisierung der Unterrichtsweise" sollten auch die Studierenden aktiv werden (Jens 2010: 320). Berühmt wurde vor allem das sogenannte „Uhlandsche Stilistikum“. Jens umschreibt es so, dass ,,jedermann Gelegenheit hatte, ein Stück seiner Wahl vorzutragen: ein Goethe-Gedicht samt Interpretation, eine Liebesgeschichte aus der eigenen Feder, eine Meditation ..." (Jens 2010: 293f.). 
In solchen Versuchen, mehr Abwechslung ins obrigkeitliche Lehren zu bringen, zeigt sich das Unbehagen einiger weniger kreativer Geister. Daran, dass es bei der Vorlesung als dominantem Lernformat blieb, änderte sich jedoch nichts. Im Rahmen des Allgemeinen Landrechts (ALR) wurden Vorlesungen in der „Universitätsverfassung in Preußen um 1860“ als zentrale Lernform etabliert. Dem Studierenden müsse es möglich sein, hieß es dort, in der vorgeschriebenen Studienzeit ,über alle Hauptdisziplinen seiner Fakultät Vorlesungen zu hören“9.

\section{Die Fortsetzung obrigkeitlicher Lehre in der Bundesrepublik}

Als die zweite deutsche Demokratie nach dem Zweiten Weltkrieg gegründet und aufgebaut wurde, war dies nicht mehr der auch noch die Republik von Weimar prägende Obrigkeitsstaat. Und doch trug die sogenannte Ära Adenauer unverkennbare Züge des Autoritären. Sie veranlassten den deutsch-jüdischen Politikwissenschaftler Karl Löwenstein die Bundesrepublik der 1950er und 1960er Jahre „demoautoritär“" zu nennen (Löwenstein 1959: 93). Insoweit nahmen die deutschen Hochschulen ihre Arbeit nach dem Krieg unter Rahmenbedingungen auf, die nicht dazu geeignet waren, hochgesteckte Anforderungen an einen anti-obrigkeitlichen Neuanfang zu erfüllen, wie sie etwa der schon genannte Walter Jens in seinem Portrait der Universität Tübingen stellte (Jens 2010: bes. 12. Kap.). Die Fortsetzung der obrigkeitlichen Lehrtradition an deutschen Hochschulen wurde zudem vor allem dadurch gestützt, dass es in den unmittelbaren Nachkriegsjahren und auch noch bis in die 1950er Jahre hinein vor allem darauf ankam, die organisatorisch-technischen Voraussetzungen dafür zu schaffen, dass in Deutschland wieder geforscht und studiert werden konnte.

\section{Die bundesrepublikanische Hochschulentwicklung als organisatorisch-quantitatives Problem}

Nicht nur für Kriegsteilnehmer, die lange entbehrte Lernmöglichkeiten um jeden Preis nutzen wollten, gehörten pädagogisch-didaktische Fragen in ein Wolkenkuckucksheim. Wenn das Fremdeln gegenüber diesem Fragenkomplex auch die spätere Entwicklung bestimmte, so lag das daran, dass die Nachfrage nach einem Ausbau der vorhandenen Forschungs- und Lernkapazitäten alle 
anderen Probleme in den Hintergrund rückte. Das lässt sich besonders gut an den Stellungnahmen und Empfehlungen des Wissenschaftsrates ablesen, der 1957 geschaffen wurde. Unter Einschluss der Forschungsförderung richtete sich seine Beratungstätigkeit vor allem darauf, jene organisatorischen und materiellen Fragen klären zu helfen, die sich bei der Expansion des westdeutschen Wissenschafts- und Hochschulsystems stellten (Bartz 2007).

Für die 1960er Jahre waren Warnungen vor einer drohenden „Bildungskatastrophe“ (Picht) und Forderungen nach „Bildung als Bürgerrecht“ (Dahrendorf) vordringlich. Dadurch wurden Diskussionen ausgelöst, die den Trend noch verstärkten, in erster Linie Fragen des quantitativen Ausbaus der Hochschulen auf die Agenda zu setzen. Wie konnte ihre Expansion gefördert und werden? In welcher Weise mussten sich die Länder in der Zusammenarbeit mit dem Bund diesen organisatorisch-materiellen Herausforderungen (,Gemeinschaftsaufgaben“) stellen?

Aufgrund beschränkter Ressourcen ging es auch - schon in den 1970er Jahren beginnend - um eine angemessene Verteilung der verfügbaren Mittel, dann in zunehmendem Maße um Einsparungen. Es musste über die Steuerung von „Studentenströmen“, über die Anpassung von Strukturen des Studiums, über Evaluation und Elite-Bildung und darüber nachgedacht werden, wie Studierende möglichst früh abschließen können. Damit ist auch der Anschluss an die jüngste Entwicklung hergestellt: Die Einrichtung von Bachelor- und Master-Studiengängen, orientiert am westeuropäischen Ausland, vollzog und vollzieht sich jenseits von Überlegungen, die mit der Frage zu tun haben, in welchen Lernformen erfolgreich gelernt werden kann.

Dem kann noch pauschal hinzugefügt werden, dass die Aufmerksamkeit studentischer Interessenvertretungen durch die Dominanz organisatorisch-materieller Probleme weitgehend gebunden war. Damit wird klar, warum sich studentische Proteste nach dem Abklingen der Studentenbewegung nur höchst

\section{Minderheitliche Widerstände gegen obrigkeitliche Lehre}

Noch bevor im Umkreis der Studentenbewegung über Lernprozesse an Hochschulen nachgedacht wurde, war es schon im studentischen Milieu des Sozialistischen Deutschen Studentenbundes zu einschlägigen Diskussionen gekommen. In der Denkschrift Hochschule in der Demokratie aus dem Jahre 1961 (SDS-Hochschul-Denkschrift 1961) wurde das traditionelle Prinzip der Einheit von Forschung und Lehre in sehr eigenwilliger Weise interpretiert. Gefordert wurde ein Lernarrangement für die Studierenden, in dem diese ,zu aktiver und gleichberechtigter Mitwirkung an der Gestaltung des Studiums in Zusammenarbeit mit den Kommilitonen und den akademischen Lehrern“ 
tet werden sollten (14). Dahinter stand eine gesellschaftskritische Position, die - auf die Hochschulen bezogen - eine passive, ,rezeptive Aneignung“ von Wissen ablehnte (15). In positiver Umschreibung dessen, was gelernt werden sollte, ging es um die Ausbildung der „geistigen Kräfte“ (13), die Entwicklung „kritischer Urteilsfähigkeit und geistiger Selbständigkeit“ (13). Die Mitarbeit der Studierenden sollte darauf hinauslaufen, ihnen die Lösung ,eigenständiger Arbeitsaufgaben“ zu übertragen (38).

Dieses Lernverständnis wirkt dann in der Studentenbewegung weiter. Es bestimmt die Forderungen, die seit dem Ende der 1960er Jahre gestellt wurden. Bei der Entwicklung eines Konzepts dialogischen Lernens wird auf sie im V. Kapitel ebenso Bezug genommen wie auf spätere hochschuldidaktische Initiativen, die seit der zweiten Hälfte der 1990er Jahre im Umkreis der Deutschen Gesellschaft für Hochschuldidaktik (DGHD) ergriffen wurden (V.2.).

Hochschulintern gab es kaum Resonanz. In der hochschulexternen Öffentlichkeit blieben die hochschuldidaktischen Forderungen so gut wie unbeachtet. Entschlossen wurde am Vorlesungsprinzip festgehalten. Das zeigt sich beispielsweise in einer Publikation des Deutschen Hochschulverbands aus dem Jahre 1991, wo es in einer der vorgelegten Thesen heißt: „Die Bildungsaufgabe der Universität verlangt die Kultur der Vorlesung.“ (Ellwein 1997: 55) Wie unangetastet die sogenannte Ordinarienuniversität in Fragen der „Lehre“ blieb, lässt sich auch fast zwei Jahrzehnte später den Empfehlungen zur Qualitätsverbesserung von Lehre und Studium entnehmen, die der Wissenschaftsrat 2008 herausgab. Zwar wich er von seiner bisherigen Linie ab, sich allein auf organisatorisch-quantitative Probleme zu konzentrieren (Wissenschaftsrat $2008^{10}$ ). Bei näherer Betrachtung seines Beitrags zur Aufwertung der „Lehre“ wird jedoch schnell klar, dass er sich in Übereinstimmung mit einer Reihe von Vorschlägen die etwa im selben Zeitraum von der Kultusministerkonferenz, der Deutschen Rektorenkonferenz und vom Stifterverband für die deutsche Wissenschaft gemacht wurden. Das greifbare Ergebnis dieser Bemühungen war ein zwischen den Regierungen von Bund und Ländern abgeschlossener „Qualitätspakt Lehre“. Für den Zeitraum 2011 bis 2020 wurden 2 Milliarden Euro zur Verfügung gestellt. Schaut man sich die Absicht, unter dem Stichwort „Qualität der Lehre“ über Lernprozesse an Hochschulen nachzudenken, etwas genauer an, so ergibt sich das Folgende:

10 http://www.wissenschaftsrat.dedpwuload/archiv/8639-08 (zuletzt überprüft: 10.08.2018). Auf die neueste Initiative des Wissenschaftsrats, ein Positionspapier vom April 2017 („Strategien für die Hochschullehre“), in dem die „Einrichtung einer eigenständigen Organisation zur Förderung und Entwicklung der Hochschullehre" vorgeschlagen wird, kann hier nicht eingegangen werden. Vgl. die Darstellung und Interpretation dieser Initiative in Borgwardt/Felmet (Hrsg.) 2018: 29-39. 
Der Begriff der „Lehre“ verweist unmissverständlich auf ein Verständnis von Lernvorgängen, bei dem es darauf ankommt, dass „Lernenden von Lehrenden beigebracht" (d. Verf.) wird, was sich Letztere als Erkenntnisse (ihrer Forschung) erworben haben. Das Vorlesungsprinzip steht für die Tradition obrigkeitlicher Lehre, dem stillschweigend jene mit ihr verbundene pädagogisch-didaktische Indifferenz zugrunde liegt, gegen die sich „,nachholende Didaktik mithilfe der Neurobiologie“ zur Geltung bringen kann. 



\section{Neurobiologische Grundlagen des Lernens}

Versucht man den neurobiologischen Zugriff auf das Lernen an Hochschulen methodologisch einzuordnen, so kann man von der philosophischen Tradition ausgehen. Diese ist überwiegend dadurch gekennzeichnet, dass Lernende als aus sich heraus agierende (Lern-) Subjekte behandelt werden. Als die Subjektphilosophie in Frage gestellt und erkannt wurde, inwiefern die (Lern-)Subjekte in gesellschaftlichen Abhängigkeiten stehen, kam es vornehmlich unter dem der Soziologie zur Sozialisations-Analyse. Diese lässt sich auf der Grundlage neurobiologischer Erkenntnisse im folgenden Sinne zu einer „Neuronalisations-Analyse“ ausbauen: Sie zielt auf Subjekte, deren Fühlen, Denken und Handeln als Ergebnis der in ihren Köpfen ablaufenden Vermittlungsprozesse betrachtet werden muss. Dabei sich diese Subjekte, die immer auch das Fühlen, Denken und Handeln lernende Subjekte sind, in Abhängigkeit von ihrer Umwelt im Allgemeinen, von den Lernmaterialien und von den Lehrpersonen im Besonderen.

Den speziellen Hintergrund einer in neurobiologischer Perspektive eingeführten Unterscheidung zwischen Nah- und Fernkommunikation bilden kommunikationstheoretische Konzepte, die in den Sozialwissenschaften entwickelt wurden. Unter anderem verweist die Handlungstheorie von George Herbert Mead auf das unvollständig bearbeitete Problem der Intersubjektivität (Nassehi 2009: 141ff.), dem eine neurobiologische Grundlage gegeben werden kann. Dasselbe trifft für sozialwissenschaftliche Ansätze zu, die unter dem von Linguistik und Semiotik diskurstheoretische Konzepte entwickelten. Sie können hier, vor allem auf Michel Foucault gestützt, eingeführt und zugleich durch neurobiologische Bezüge angereichert werden. Wenn im Folgenden auf Lernende Bezug genommen wird, so ist - wie in der Einführung (2.) erläutert immer deren Beziehung zu den Mit-Lernenden mitgemeint, auch wenn diese nicht in die Analyse einbezogen wird.

Zum Einstieg in die hier konzipierte „Neuronalisations-Analyse“ bedarf es einer Reihe von Grundkenntnissen über den neuronalen Lernapparat (1.). Diese müssen durch spezielle Kenntnisse darüber ergänzt werden, dass ertragreiches Lernen von emotionalen Schubkräften abhängig ist, die in Nahkommunikation wirksam sind (2.). Schließlich muss in neurobiologischer Perspektive verstanden werden, dass Lernprozesse im Rahmen von Machtbeziehungen ablaufen (3.). 


\section{Grundkenntnisse über den neuronalen Lernapparat}

\section{Wie Gehirn und Körper zusammenarbeiten: ,Embodied Learning“}

Eine grundlegende Einsicht der Neurobiologie bezieht sich darauf, dass Lernende ihre eher große oder eher geringe Lernbemühung durch die Haltung ihres gesamten Körpers, durch Bewegungen ihrer Gliedmaßen und speziell durch ihren Gesichtsausdruck begleiten. Diese enge Zusammenarbeit zwischen Gehirn und Körper hat Antonio Damasio in einer zuspitzenden Präsentation des Ergebnisses langjähriger Forschungen auf die Formel gebracht, wir alle hätten „unseren Körper im Kopf“ (Damasio 2011: 118). Damit ist gemeint, dass Gehirn und Körper miteinander vernetzt sind. Der Körper vermittelt dem Gehirn Informationen über Körperzustände, die auf äußere Einwirkungen zurückgehen. In neuronalen Regionen, die dafür zuständig sind, werden diese Informationen registriert und in dort angelegten Karten eingetragen.

Auf diese Weise kommt es in der Zusammenarbeit zwischen Körper und Gehirn zur Verarbeitung sich verändernder Körperzustände. Da durch diese wiederum andere Körperteile betroffen sind und indirekt speziell für sie zuständige Gehirnregionen erreicht werden, entstehen ,Resonanzschleifen“ (Damasio 201

\section{Wie Sinnesreize aufgenommen und in Schaltkreisen verarbeitet werden}

Der neuronale Apparat ist elementar dafür zuständig, dass von außen kommende Signale aufgenommen und verarbeitet werden. Aus der Fülle der auf die unterschiedlichen Sinne einwirkenden Impulse werden - selektiv - diejenigen ausgewählt, die sich mit besonders großer Intensität (Salienz) aufdrängen (Koch 2005: 173-176).

In seinem Buch zur Erforschung des Unbewussten in Kunst, Geist und Gehirn von der Wiener Moderne bis heute (= Untertitel von: Das Zeitalter der Erkenntnis, Kandel 2012) hat der Neurobiologe Eric Kandel die Aufnahme und Weiterleitung von Signalen am Beispiel der Verarbeitung visueller Reize illustriert. Dabei kommt es nicht auf Einzelheiten des Informationstransports und auf die genauen Bezeichnungen seiner Stationen an. Vielmehr geht es darum, einen groben Eindruck von der Komplexität eines Systems neuronaler Schleifen zu gewinnen, die vielfach miteinander verknüpft sind. Die von Kandel beschriebenen Abläufe stehen dafür, ,dass jeder geistige Prozess - perzeptueller, emotionaler und motorischer Art - von ganz unterschiedlichen Gruppen spezialisierter neuronaler Schaltkreise abhängt, die hierarchisch geordnet in 
Am Anfang des Weges, den die Informationen in der beispielhaft gewählten Schleife des Sehsystems durchlaufen, steht die Netzhaut des Auges. Es geht weiter ,im seitlichen Kniehöcker des Thalamus, darauf in der primären Sehrinde im Okzipitallappen (= Hinterhauptlappen; d. Verf.) und schließlich in weiteren 30 Arealen im Okzipital-, Temporal- und Frontallappen (= Hinterhaupt-, Schläfen- und Stirnlappen; d. Verf.) der Großhirnrinde“ (Kandel 2012: 277).

Von entsprechender Komplexität sind die Wege und Stationen des Sehsystems, über die es zum Anschluss an die Areale kommt, die für den Spracherwerb zuständig sind. Dieser ist eine zentrale Funktion des Gehirns. Sie kann auch vom komplizierten Gehörorgan her realisiert werden und mit dem Sehvorgang kombiniert sein. Umgekehrt bestehen komplexe neuronale Voraussetzungen für Prozesse absichtlichen Sprechens. Dabei geht es darum, inhaltlich passende Wörter zu sie - bei Sätzen - grammatikalisch korrekt zu verknüpfen und mithilfe sprechmotorischer Werkzeuge zu artikulieren (Roth 2011: 207ff.).

Zur einigermaßen vollständigen Beschreibung der zum großen Teil unbewussten Abläufe, in die auch der Bewegungsapparat einbezogen ist, gehört die emotionale Ablaufkomponente: Die Zuständigkeit für die emotionale Bewertung eingehender Informationen liegt im sogenannten limbischen System. In dieses eingefügt sind Belohnungsstrukturen. Zu ihnen gehören die Antriebskräfte des Botenstoffs Dopamin. Dopaminerge Neuronen (besonders im ventralen tegmentalen Areal, im Nucleus accumbens sowie im ventralen Striatum angesiedelt) sind einerseits für Belohnungserwartungen zuständig. Sie werden andererseits aktiv, wenn diese Erwartungen enttäuscht werden.

Auch wenn der Bezug zu den hier interessierenden Lernvorgängen nicht besonders wichtig erscheint, wäre es falsch, die Beteiligung des Geruchssinns gering zu schätzen. Entwicklungsgeschichtlich ist er der älteste Sinn, der die Ausgestaltung der menschlichen Kommunikation maßgeblich

(vgl. zum Folgenden besonders Hatt \& Dee 2012 und im Internet zugängliche Interviews des Geruchsforschers Hanns Hatt). Die Geruchsforschung kann beispielsweise zeigen, dass gelingende Liebesbeziehungen davon abhängig sind, dass die von Partnern ausgehenden und von ihnen aufgenommenen Düfte - die von ihnen ausgebrachten Duftmoleküle - zusammenpassen. Zu beobachten ist, dass Düfte und ihre Verarbeitung starken kulturellen unterliegen. Dabei sind alle Prozesse, in denen Gerüche verarbeitet werden, dem Bewusstsein weitgehend entzogen.

Über die je besondere Ausgestaltung der Riechbeziehungen entscheidet einerseits das einzigartige das jedes (lernende) Individuum mitbringt. Andererseits kommt es darauf an, wie die individuellen Duftmarken in der Nase des Gegenübers verarbeitet werden. Diese ist mit 350 Arten von Riechzellen ausgestattet, die für vielfältige Düfte zuständig sind. Dabei ist wichtig, 
dass die millionenfach vorhandenen Riechzellen der Nase in Monatsfrist jeweils ausgetauscht werden. Auf Duftmoleküle unterschiedlichster Art reagieren auch spezielle Duftsensoren, die beispielsweise in der Haut, im Magen und an vielen anderen Stellen des Körpers platziert sind. Wo immer sie auch aufgenommen werden, gilt für alle Duftimpulse, dass sie auf direktem Wege zum Riechhirn weitergeleitet werden und daher mit den vielfältig verzweigten Prozessen des erwähnten limbischen Systems, beispielsweise mit dem Belohnungssystem, vernetzt sind.

In enger Verbindung zum exemplarisch beschriebenen Sehsystem stehen die Prozesse des Lesens und Lesenlernens. Dabei handelt es sich um einen Lernvorgang, der seinen hohen Lernertrag daraus gewinnt, dass er das neuronale Netzwerk des Gehirns in großer Breite, Tiefe und Dichte erschließt (Dehaene 2010). Dieser Lernansatz wird im V. Kapitel als Beispiel für „Lernen ohne Digitales“ noch genauer umschrieben, um vergleichend den Ertrag des „Lernens mit Digitalem“ abschätzen zu können (V.1.).

Die überblickshafte Darstellung des neuronalen Mechanismus von Lernvorgängen, eingebettet in ein weit verzweigtes neuronales Schleifensystem, muss an einem speziellen Punkt noch erweitert werden. Dabei kann an die Vulgärpsychologie angeknüpft werden.

\section{Wie sich bewusstes von unbewusstem Lernen unterscheidet}

Mit dem Namen Sigmund Freud sind vulgärpsychologische Vorstellungen verbunden, nach denen es Verhaltens- und insbesondere Sprechweisen (,Freud'scher Versprecher") gibt, die auf unlenkbare und unbewusst wirksame Antriebe zurückgehen. Seit es Techniken - vor allem bildgebende Verfahren - gibt, die Freund noch nicht zur Verfügung standen, ist es ein Gemeinplatz der Neurobiologie, dass die neuronalen Mechanismen zum allergrößten Teil unbewusst ablaufen (Rösler 2011: 313ff.). Die zentrale Frage, wann und an welchen Schwellen es dazu kommt, dass die neuronale Verarbeitung von Informationen von Unbewusstheit in Bewusstheit umschlägt, ist zwar noch nicht befriedigend geklärt. Es gibt jedoch eine Reihe von Signaturen - ein in der neueren Bewusstseinsforschung inzwischen etablierter Begriff (v. a. Dehaene 2014: 4. Kap.) -, die den Unterschied zwischen unbewusster und bewusster Verarbeitung erkennen lassen.

Details dieser Erkenntnisse können hier nicht wiedergegeben werden. Es müssen Hinweise auf die zentralen, in der (Groß-)Hirnrinde liegenden Schaltstationen einerseits, auf die Synchronisierung dieser Stationen andererseits genügen. Zum einen zeigt sich, dass bewusster und damit bewusst ins Gedächtnis Information unter folgenden Voraussetzungen möglich ist: 
Bewusst werden von den Sinnesorganen aufgenommene Reize immer dann, wenn es dazu kommt, dass in den Unterabschnitten des schon bezeichneten präfrontalen Cortex und die in den Teilregionen des parietalen Cortex ausgelösten lokalen Aktivitäten ineinander übergehen. Schon Antonio Damasio hat dies betont und dafür die eingängige Formel gefunden, dass ,integrierter Geist aus parzellierter Aktivität" hervorgeht (Damasio 2006: 137-139). Zum anderen und zugleich bedarf es der bewusstseinsbildenden elektro-magnetischen Synthese der lokalen Aktivitäten in den genannten Regionen und darüber hinaus ihrer Verbindung zu weiter entfernt liegenden Regionen des neuronalen Netzwerks.

Es ist diese elektromagnetische Signatur bewusster Verarbeitung von Informationen, die erst erforscht werden kann, seit sich am Kopf von Probanden Elektroden oder Magnetsensoren befestigen lassen (Dehaene 2014: 178). Auf diese Weise lassen sich elektro-magnetische Wellen unterschiedlichsten Typs und unterschiedlicher Schnelligkeit messen. Es sind diese Signaturen, die anzeigen, inwieweit die für Bewusstheit und indirekt für bewusstes Lernen notwendige Synthese erreicht wird.

Im Folgenden lässt sich auf den Unterschied zwischen einer Gedächtnisbildung verweisen, die auf bewusstem Lernen beruht, und einer solchen, bei der Lernergebnisse unbewusst aufgezeichnet werden. Im ersten Fall liegt eine „Lernsynthese“ vor, an der erkennbar ist, dass ihre - hier in der Hochschulperspektive interessierende - Qualität davon abhängig ist, wie hirngerecht die Lernprozesse angelegt sind.

Für die hier interessierende Betrachtung des Gehirns als Lernapparat ist der Vorgang des Lesens und Lesenlernens mit traditionellen Print-Materialien von besonders offensichtlicher Bedeutung. Im V. Kapitel wird er im Vergleich mit der Verarbeitung von digitalen Materialien noch näher beschrieben (V.1.).

\section{Gedächtnisbildung}

Anknüpfend an vielfache Versuche, die neuronale Struktur der Erinnerungsund Gedächtnisbildung aufzugliedern (Roth 2003: 153ff.), müssen unter dem Gesichtspunkt des neuronalen Lernpotentials verschiedene Arten von Gedächtnis hervorgehoben werden. Das semantische Gedächtnis wird, da seine Inhalte, um deren Verarbeitung die denkenden und handelnden Individuen wissen, ,gut" oder ,schlecht" wiedergegeben werden können, auch deklaratives Gedächtnis genannt. In ihm eingetragen werden zum einen Ereignisse und Objekte in der Natur (z. B. Naturkatastrophen). Zum anderen werden Vorgänge aufbewahrt, die das gesellschaftliche System kleiner und großer Institutionen hervorbringt. Die Aufzeichnungen umfassen immer auch die Konzepte, und 
das heißt auch die sprachlichen Mittel, die den Zugriff auf Natur und Gesellschaft ermöglichen (Kandel u. a. 2000: 1235). Soweit es um die neuronale Eintragung von Erlebnissen geht, die - in allgemeine „events“ eingebettet - den speziellen Lebensweg einzelner Menschen berühren, wird das semantische Gedächtnis zum sogenannten autobiographischen Gedächtnis (auch: episodisches Gedächtnis). Auch diese Informationen des Erlebnishaushalts einzelner Personen werden zumindest teilweise bewusst aufgenommen.

Demgegenüber gibt es Gedächtnisinhalte, die sich stillschweigend herausbilden und daher - im Unterschied zum deklarativen Gedächtnis - einem impliziten Gedächtnis zugeordnet werden. Dieses schließt einen neuronalen Zielbereich ein, der prozedurales Gedächtnis genannt wird. Hier kommt es einerseits zu stillschweigend aufgezeichneten motorischen Fähigkeiten. Andererseits können diese aber auch - wie beispielsweise Fahrradfahren und Autofahren - geplant entwickelt werden. Dasselbe gilt etwa für bestimmte rhetorische Fertigkeiten, die sich im Rahmen unbewusst entstandener sprachlicher Fähigkeiten einüben lassen.

Ebenfalls zu den tendenziell implizit verarbeiteten Informationen gehören emotionale Bewertungen. Sie begleiten alle Formen von Gedächtnisbildung und schaffen einen Komplex emotionalen Gedächtnisses, der für die hier interessierenden Lernvorgänge von besonderer Bedeutung ist.

Eingebettet sind diese Mechanismen der Gedächtnisbildung in eine allgemeine neuronale Grundfähigkeit, sich gegenüber der Umwelt aufgeschlossen zu zeigen. Schon bei Kleinkindern, die in ihrer (Groß-)Hirnrinde noch über keinen für Planung zuständigen präfrontalen Cortex verfügen, sticht diese ungesteuerte Offenheit und Wachheit besonders ins Auge. Auf dieselbe Grundfähigkeit verweisen die Überblicksbilder, die von Erwachsenen-Gehirnen liefern. In ihrer „Quirligkeit“ und Hab-Acht-Stellung zeigen diese Bilder eine umfassende neuronale Lebendigkeit, die Voraussetzungen dafür schafft, dass Sinnesreize, ohne dass spezielle Lernabsichten bestehen müssen, durch die zuständigen neuronalen Areale aufgenommen, verarbeitet und verbreitet werden.

Wie sich im III. Kapitel zeigen lässt, wird die allgemeine Lern-Konstellation an den Hochschulen unter anderem durch einen allgegenwärtigen Mythos der Wissenschaft und durch Verhaltenscodes bestimmt, die unkalkuliert in das Gedächtnis eingehen. Adressiert werden vor allem das und das prozedurale Gedächtnis der Lernenden. Für sie fallen insbesondere Erfahrungen ins Gewicht, die sich mit der generell praktizierten Unterordnung gegenüber denjenigen verbinden, die das Lernen anleiten. Speziell im disziplinierenden Prüfungs- und Notensystem und bei der Vermittlung von

Arbeitstechniken wird prozedural gelernt: Wie wird argumentiert? Wie werden schriftliche Arbeiten gegliedert? Wie wird zitiert? 
Soweit Lernende aus eigenem Antrieb ein Hochschulstudium aufnehmen, geht es ihnen darum, gestützt auf einen Sockel allgemeiner neuronaler Wachheit, Lebendigkeit und Lernbereitschaft, in ausgewählten Fächern gezielt das dort etablierte Wissen zu erschließen. Aus neurobiologischer Sicht wollen sie im Netz ihres semantischen Gedächtnisses Erinnerungen aufbauen, die als Wissen abgerufen werden können. Unter dem Gesichtspunkt der hier interessierenden Lernformate und speziell im Blick auf den im IV. Kapitel beschriebenen Vorlesungsbetrieb geht es um die Frage: Inwiefern ist dieser geeignet, eigenständiges Lernen zu stützen und bei den Lernenden das zu entwickeln, was einleitend orientiert an Searle - „subjektive Intentionalität“ und, auf die Studierenden als kommunikative Einheit bezogen, „kollektive Intentionalität“" genannt wurde.

Zur subjektiven und kollektiven Zielsetzung der Studierenden gehört auch, dass sie absichtsvoll in eine Lebensphase eintreten, in der sie auf Lernerfahrungen hoffen, die zur individuellen Formung ihrer Persönlichkeit beitragen. Das wiederum heißt neurobiologisch, dass in ihr autobiographisches Gedächtnis Fähigkeiten eingetragen werden sollen, die in der pädagogischen Psychologie als Merkmale sozialer Kompetenz gewertet werden.

\section{Vom Kurzzeitgedächtnis zum Langzeitgedächtnis}

Was die Ausformung des individuellen, unkalkuliert und kalkuliert aktivierten neuronalen Lernapparats angeht, so lassen sich drei miteinander verbundene Bereiche unterscheiden: Ein Eingangsbereich, ein Zwischenbereich und ein Zielbereich. Letzterer wurde schon erwähnt. Er wird Langzeitgedächtnis genannt, weil die eingehenden Informationen

und auf Dauer aufbewahrt und abrufbar gehalten werden. Dabei sind der Hippocampus und die Neuronenverbände seiner Umgebung maßgeblich beteiligt. Die zwischen den aufnehmenden Zellverbänden ablaufenden elektromagnetischen Oszillationen sorgen für eine Vereinheitlichung der aufgebauten und immer wieder angereicherten Erinnerungs- bzw. Wissensbestände. Neurophysiologisch folgt die Wissensproduktion den sogenannten Hebb'schen Regeln synaptischer Verstärkung, deren Gültigkeit sich immer wieder bestätigt hat.

Dem Zielbereich der Lernvorgänge steht ein Eingangsbereich gegenüber, der Kurzzeitgedächtnis und im Blick darauf, dass Informationen kurzzeitig behalten und bearbeitet werden, auch Arbeitsgedächtnis genannt wird. Neuere Untersuchungen betonen, dass Lernende durch eigene Anstrengung daran mitwirken, ihre Aufmerksamkeit zu mobilisieren (Cowan: „Embedded Processing Model“). Insoweit ist zu berücksichtigen, dass sich an unkalkulierte Sinneswahrnehmungen „Effort-Mechanismen“ anschließen (Birbaumer/Schmidt 2010: 500-502). Zuständig sind neuronale Strukturen, die hauptsächlich im schon genannten dorsolateralen Cortex der (Groß-)Hirnrinde liegen. 
Folgt man den neueren Forschungsergebnissen, so kommen bei Lernenden nur Informationen an, die - im Verhältnis zum schon aufbewahrten Wissensund Erinnerungsbestand - neu sind. Haben Informationen solchen Neuigkeitswert, so sie über die einzelnen Sinnesorgane kanalisiert Zugang. Im Zeitraum von wenigen Millisekunden werden von außen kommende Informationen „roh verarbeitet“ (Birbaumer/Schmidt 2010). Es eine Art von Konkurrenzkampf zwischen verschiedenen Modalitäten der Sinne statt, deren Ressourcen beschränkt sind. Das aber heißt, dass sich die Aufmerksamkeit konkurrierend entweder darauf richtet, die Zahl der Informationen groß zu halten oder aber einzelne Informationen besonders intensiv aufzunehmen. ${ }^{11}$ Das gesamte Zeitfenster, in dem das Kurzzeitgedächtnis Informationen aufnimmt, liegt bei 1-3 Sekunden. Der Umfang der Informationen, die in diesem zeitlichen Intervall verarbeitet werden können, liegt bei ungefähr $7+/-2$ Informationseinheiten. Durch Verdichtung (,,chunking“) ist eine - wenngleich sehr begrenzte - Steigerung dieses Umfangs möglich. Die im Kurzzeitgedächtnis ankommenden Informationen halten sich etwa 15-20 Sekunden. Kommen neue Inputs hinzu, so verblassen und zerfallen sie. ${ }^{12}$ Man sollte also insgesamt von einer sowohl zeitlich als auch inhaltlich eng beschränkten Aufnahme- und Haltefähigkeit des Kurzzeitgedächtnisses ausgehen. Diese muss bei der hier interessierenden Frage, welche Erträge das Lernformat Vorlesung liefert, besonders beachtet werden.

Für den Lernvorgang und im Besonderen für das hier interessierende Lernen im Vorlesungsbetrieb von Hochschulen ist entscheidend, was sich in den neuronalen Netzverbindungen abspielt, die das Kurzzeitgedächtnis mit dem Langzeitgedächtnis verknüpfen. Unter Beteiligung von Neuronengruppen, die hauptsächlich im Scheitellappen (parietaler Cortex) und im Schläfenlappen (temporaler Cortex) liegen, kommt es für ertragreiche Erinnerungs-/Wissensproduktion auf Folgendes an: Die über die Sinne eingehenden Informationen müssen wiederholt dargeboten werden (Konsolidierung), um in den beteiligten neuronalen Netzen einen hohen Schwellenwert der Erregung zu erzeugen. Nur dann besteht eine Chance, dass die Impulse weitergegeben, zusammengefügt und als Erinnerung bzw. Wissen dauerhaft aufbewahrt werden.

Unter dem hier interessierenden Gesichtspunkt des Vorlesungsbetriebs an Hochschulen ist aber nicht nur der Neuigkeitswert von Informationen von Belang. Genauso wichtig ist, dass die neuen Informationen auf schon vorhandene Erinnerungs- bzw. Wissensbestände stoßen, an die sie anknüpfen können.

11 Dieser Punkt der Konkurrenz um beschränkte Aufmerksamkeit wird besonders herausgearbeitet von: Roth 2011: 132f.

12 Woolfolk 2008: $316 f f$. mit einer Zusammenfassung einschlägiger Literatur. 
Stimmt diese Darstellung, so wird zugleich deutlich, dass die Unterscheidung zwischen Kurz- und Langzeitgedächtnis nur analytische Bedeutung hat.

Um den Aufbau des Lernapparats und die Dynamik der Abläufe des Lernens - vor allem wiederum im Blick auf Vorlesungen - vollständig zu beschreiben, müssen Forschungsergebnisse berücksichtigt werden, die darauf verweisen, dass die vergleichende Bewertung ankommender Informationen mit emotionalen Faktoren gekoppelt ist. Über die Emotionsforschung, die zuerst von Joseph LeDoux (LeDoux 1996/dt. Übers. 1998) und dann vor allem von Antonio Damasio (Damasio 2003/dt. Übers. 2005; Damasio 2010/2011) geprägt wurde, gibt der folgende Abschnitt über die emotionalen Schubkräfte ertragreichen Lernens Auskunft (2.). Zuvor muss aber noch kurz auf automatische Imitation eingegangen werden.

\section{Imitation und Imitationslernen}

Aus der sozialpsychologischen Forschung ist Imitation als Mimikry bekannt. wird in Nahkommunikation Gähnen, Kratzen oder Lachen nachgeahmt. Diese Art der ansteckenden Imitation wurde in verlässlichen Studien schon bei Säuglingen beobachtet, die, kaum geboren, Zungen- oder Mundbewegungen der Eltern nachahmen (Meltzoff/Decety 2003: 110-112). Auch gehört „reaktives Weinen“ zum Repertoire automatischer Imitation.

Sie folgt dem Muster der klassischen und operanten Konditionierung, wie sie in vielfältigen Experimenten der Verhaltensforschung illustriert wurde (Roth 2011: 94ff.). Nach dem neueren Forschungsstand wird die automatische Imitation, vor allem in Experimenten mit Makaken und Schimpansen belegt, mit Aktivierungen der sogenannten Spiegelneuronen in Verbindung gebracht, die im prämotorischen Cortex, im unteren Scheitellappen, in der Nähe des Broca-Areals und in der Furche des Schläfenlappens nachgewiesenen wurden (Verplaetse 2011: 43).

Im hier interessierenden Lernfeld der Hochschulen kommt es zweifellos $\mathrm{zu}$ automatischer Imitation von Vortrags- und Diskussionsstilen, begleitet von charakteristischer Körpersprache. Leider fehlt es an einschlägigen Forschungsbemühungen. Unverkennbar ist aber, dass automatische Imitation nicht im Zentrum ertragreicher Lernprozesse steht. 


\section{Ertragreiches Lernen durch emotionale Schubkräfte in Nahkommunikation}

\section{Emotionale Bedürfnisse nach Nahkommunikation}

Auf den amerikanischen klinischen Psychologen A. Maslow geht die Unterscheidung zwischen materiellen und nicht-materiellen Grundbedürfnissen zurück (Maslow 1954), die in vielen Forschungsbereichen fruchtbar gemacht wurde (u. a. Galtung 1994). Bei den materiellen Bedürfnissen handelt es sich auf unterster hierarchischer Stufe um überlebensnotwendige Nahrung, Kleidung, medizinische Versorgung und Ausbildung. Auf den nicht-materiellen Stufen geht es um Bedürfnisse individueller Selbstentfaltung, die ihren Bedürfniskern in der Erwartung mitmenschlicher Nähe, Zuwendung und Liebe haben. Neurophysiologisch wird dieser Bedürfniskern von zuständigen Abschnitten der Amygdala und des sogenannten mesolimbischen Systems getragen.

Belegen lässt sich, dass es für die Intensität und die Dauerhaftigkeit des Ergebnisses von Lernvorgängen auf mitmenschliche Nähe ankommt. Diese stellt sich zwischen denjenigen, die das Lernen anleiten, und denen ein, die sich - unter Einschluss der Beziehungen zu den jeweils Mit-Lernenden - zum Lernen anleiten lassen. Die Evolutionsbiologie unterstützt diese Einsicht. Sie fand heraus, dass Lernprozesse, wie sie aktuell ablaufen, in der Tradition des Lernens in Stammesgesellschaften stehen. Deren Überleben hing von genetischen Grundlagen und davon ab, dass die Mitglieder von Kleingruppen in der neuro-biologischen Expression der Gene Voraussetzungen für kooperatives Verhalten entwickelten. Die kooperative Nahkommunikation entschied darüber, ob genügend Nahrung beschafft und Feinde abgewehrt werden konnten. ${ }^{13}$ Darüber hinaus müssen evolutionsbiologische Erkenntnisse ernst genommen werden, nach denen Gehirne in ihrer Entwicklungsgeschichte so ausgestaltet wurden, dass sich die Kooperationsverhältnisse zwischen Verwandten, Lebenspartnerinnen und -partnern sowie befreundeten Personen enger ausformten als die eher kompetitiv geprägten Beziehungen zu Fremden (Pinker 2003: bes. 342f.). Die vorliegende Empirie verweist darauf, dass „,nepotistischer Altruismus“ nicht notwendig ungetrübte Harmonie unter „loved ones“ und

13 Vgl. zum Folgenden: Franz M. Wuketits' Hinweise auf eine in der Evolution angelegte menschliche Neigung zu kooperativem Verhalten als möglichen Anknüpfungspunkt einer biologisch fundierten Moralauffassung: Ders., Evolutionäre Ethik und ihre Kritiker. Versuch einer Metakritik, in: Wilhelm Lütterfelds/Thomas Mohrs (Hrsg.), Evolutionäre Ethik zwischen Naturalismus und Idealismus. Beiträge zu einer modernen Theorie der Moral, Darmstadt 1993: 228-231. 
extreme Disharmonie in den Beziehungen zu Fremden bedeutet. Zwischen Spannungen unter Ersteren (vgl. Pinkers Bezugnahme auf 346-351) und der angespannten Distanz zu fernstehenden Menschen gibt es jedoch deutliche Unterschiede. Daher muss Kooperation in Nahkommunikation von Kooperation in Fernkommunikation unterschieden werden.

Bezieht man diese Beobachtungen auf den neuronalen Lernapparat der Mitglieder von Kleingruppen, so war ihr Überleben davon abhängig, dass sie in Nahkommunikation voneinander lernten, was gelernt werden musste, um die existenzsichernde Nahkommunikation aufrechtzuerhalten. Seit in groß-

Gesellschaften moderne Informationstechniken verfügbar sind, hat diese Nahkommunikation sowohl im institutionalisierten Schul- und Hochschulbetrieb als auch in außerschulischen Lernfeldern Konkurrenz durch fernkommunikatives Lernen bekommen. Lernbeziehungen werden über Funk und Radio, über das Fernsehen und aktuell vor allem über das Internet hergestellt (vgl. das V. Kap.). Fern-Unterricht, Fern-Studium und damit Fern-Universitäten wurden möglich. Da aber kein Zweifel darüber besteht, dass - allen telekommunikativen Neuerungen zum Trotz - sich beim Übergang zu den Großder neuronale Lernapparat der Gesellschaftsmitglieder in seinen elementaren Eigenschaften nicht geändert hat, so folgt daraus: Das Nachdenken über Lernprozesse muss zwar die Bereicherung durch fernkommunikatives Lernen einbeziehen. Daran, dass ertragreiches Lernen vor allem in Nahkommunikation erfolgt, hat sich indessen nichts geändert.

\section{Emotionale Bindungen (Attachment)}

Der Grundstock ertragreichen Lernens besteht aus engen affektiven Bindungen zwischen denjenigen, die das Lernen anleiten, und denen, die sich - unter Einschluss der Mit-Lernenden - individuell zum Lernen anleiten lassen. Wie wichtig diese Bindungen sind, lässt sich beobachten, wenn sie in missglückten Biographien fehlen und wenn bestehendes Attachment zerstört wird.

Erste einschlägige Forschungen stammen von dem Psychoanalytiker René Spitz, der in den 1940er Jahren die Entwicklung von Kindern, die im Findlingsheim ungenügend betreut wurden, mit Kindern verglich, die in einer Gefängniskrippe aufwuchsen, dort aber von ihren Müttern tägliche Zuwendung bekamen. Wie in späteren Untersuchungen ähnlicher Anlage ergab die Vergleichsbetrachtung, dass es von günstigen oder ungünstigen Kontakten zu nahestehenden Menschen abhängt, ob und bis zu welchem Grade die für affektive Kommunikation zuständigen neuronalen Areale ausreifen. ${ }^{14}$

14 Vgl. die Zusammenfassung und Kommentierung der Ergebnisse bei: Kandel u. a. 2000: 1128. 
Welche Bedeutung affektiver Nähe zukommt (vgl. überblicksartig: Birbaumer: 146f.), wird auf der Verhaltensebene durch Reaktionen auf besonders einschneidende Trennungserlebnisse belegt. Insbesondere wenn Kinder sterben, stürzen die mit ihnen eng verbundenen Eltern in tiefe $\mathrm{V}$

Sie bleiben sehr lange und oft ein Leben lang untröstlich verletzt. Neurobiologisch aufgebaut wird starkes mitmenschliches Attachment vor allem durch den Botenstoff Oxytozin (Bauer 2007: 44ff.). Er ist in spezieller Weise im Mutter-Kind-Verhältnis wirksam, indem er den Geburtsvorgang und danach das Ausscheiden von Muttermilch unterstützt. Im Hypothalamus produziert, fördert er einerseits den Aufbau von Bindungen. Andererseits trägt er - zum Teil im Zusammenwirken mit Dopamin - dazu bei, dass mit Bindungserfahrungen Wohlgefühl einhergeht.

Welcher Lernschub mit solchem Wohlgefühl verbunden sein kann, wissen Schülerinnen und Schüler, die von Lehrpersonen besondere Zuwendung erfahren haben. Rückblicke auf die Schulzeit sind voll von Erzählungen, die auf solche Erfahrungen verweisen. Komplementär dazu wird über herablassend gleichgültiges Verhalten von Lehrerinnen und Lehrern berichtet, das im Gedächtnis der betroffenen Schülerinnen und Schüler tiefe Spuren hinterlassen hat.

Was akademische (Lern-)Karrieren angeht, so gibt es variantenreiche Erzählungen von Absolventinnen und Absolventen, die ihren Erfolg darauf zurückführen, dass sich ihre Wege mit Professorinnen und Professoren kreuzten, von denen sie sich nicht nur fachlich, sondern vor allem menschlich betreut fühlten. Beispielsweise galt das für den Berliner Politologen Ernst Fraenkel, der von „Spaziergang-Gesprächen“ erzählt, die er mit dem berühmten Rechtsgelehrten Hans Kelsen führen durfte. Für Fraenkel waren diese eine unverzichtbare Voraussetzung dafür, dass er sich als diskriminierter Akademiker jüdischer Herkunft nie - auch in der Emigration nicht - davon abbringen ließ, seinen Weg als juristischer Außenseiter zu gehen.

Dasselbe gilt für Nobelpreisträger Eric Kandel. Als Kind einer jüdischen Emigrantenfamilie schaffte er es nur deswegen ans Harvard College und über dieses an die Harvard-Universität, weil ihn ein Geschichtslehrer unter seine Fittiche nahm und unermüdlich unterstützte. Auch Kandels spätere Karriere als medizinischer Psychologe wäre nicht denkbar gewesen, wenn ihm nicht wie er erzählt - „wunderbare und warmherzige Menschen“ geholfen hätten (Kandel 2007: Unterkapitel „Erziehung in Amerika“").

Dazu passt, dass der Verfasser selbst - Anfang der 1960er Jahre ein „kleiner Student" an der Tübinger Universität - dadurch einen massiven Anstoß zum intensiven Studium der Polikwissenschaft bekam, dass er durch Theodor Eschenburg, einen besonders charismatischen Vertreter seines Faches, mehrfach dazu aufgefordert wurde - es war ganz banal -, ihn auf einem nur Eschenburg vorbehaltenen Schleichweg von seinem Seminargebäude zum Hörsaal zu 
begleiten. Es ist Attachment schon dieser einfachen Art, das Lernbereitschaften anregt und unterstützt.

\section{Empathische Beziehungen}

Empathische Beziehungen bauen auf Attachment auf. Sie sind von der Forschung ebenso wie ihre neurobiologischen Grundlagen breit untersucht worden (Verplaetse 2011: 36ff.). Belegt wurde die Fähigkeit, sich in die Lage von Partnerinnen und Partnern zu versetzen, vor allem für den gegenseitigen Nachvollzug von Erfahrungen des Schmerzes, auch des Ekels. Zur ermittelten „Schmerz-Matrix“, den aktiv werdenden neuronalen Arealen, gehören vor allem der ACC (Anteriorer Cingulärer Cortex) und die Insula (Anteriorer Insulärer Cortex). Auf Lernbeziehungen übertragen geht es um soziale Resonanz. Im Gegensatz zur beschriebenen automatischen Imitation steckt im gegenseitigen Mitschwingen absichtsvolle Imitation. Insoweit hat „Lernempathie“ - man könnte sagen - etwas „Kapitulierendes“. In empathischen Verhältnissen gibt es Spielräume, in denen Imitation bis zu einem bestimmten Grade kontrolliert 15

Vor allem Gestik und Augenbewegungen sind integraler Teil empathischer Beziehungen. Daher schließen diese auch ein visuelles Moment ein. Dabei sind insbesondere die obere Furche des Schläfenlappens (Sulcus temporalis) und der untere Schläfenlappen (Gyrus fusiformis) beteiligt (Birbaumer/Schmidt 2010: 794).

$\mathrm{Zu}$ differenzieren ist zwischen intellektueller Empathie zum einen und hier besonders interessierender - emotionaler Empathie zum anderen ("theory of mind“/,mentalizing“). Diese Unterscheidung hat damit zu tun, dass für die Fähigkeit, sich in die emotionale Lage nahestehender Menschen hineinzuversetzen, andere neuronale Netzwerkbereiche zuständig sind als für den nüchternen Nachvollzug von Überzeugungen, Absichten und Erfahrungen fernstehender Menschen (Singer 2006). Nehmen wir das schon erwähnte Beispiel

15 Für spezielle Interaktionen zwischen (Lern-)Subjekten sind im Gehirn bestimmte Teil-Netzwerke zuständig. Sie machen das Gehirn, wie es hypothetische Betrachtungen von Antonio Damasio nahelegen (Damasio 2010: 113ff.), zum „Als-ob-Apparat des Körpers“. Für je spezielle Bereiche der Interaktion können spezielle Neuronengruppen über „Als-ob-Körperschleifen“ sowohl den jeweils eigenen Köperzustand eines Subjekts als auch den eines Interaktionspartners simulieren, bevor sich dieser Zustand tatsächlich einstellt. Auf diese Weise kann sich das einzelne Subjekt auf künftige Aktivitäten vorbereiten. In den Beziehungen zwischen (Lern-) Subjekten ist eine Vorausschau auf gemeinsam durchzuführendes oder zu verhinderndes Handeln möglich. Dabei geht es im Besonderen um die subjektive und intersubjektive Vorabklärung der emotionalen Anteile subjektiven oder interaktiven Verhaltens. 
der um Kinder trauernden Eltern. Wer die Eltern kennt, wird durch einfühlende Anteilnahme emotional stark bewegt sein. Demgegenüber kommt es bei der Einfühlung in Eltern, über deren Unglück irgendwo in den Medien berichtet wird, zu eher ,coolen“ Reaktionen. ${ }^{16}$

Überträgt man diese Beobachtungen auf den Problemkreis ertragreichen Lernens, so heißt das: Lernende, die ihren Lernstoff aus nächster Nähe angeboten bekommen, nehmen ihn affektiv-empathisch auf, wogegen aus der Ferne eingeleitete Lernvorgänge überwiegend von intellektueller Empathie bestimmt sind. Damit haben aber die in Nahkommunikation vermittelten Informationen eine größere Chance, im Langzeitgedächtnis eingetragen zu werden, als der in Fernkommunikation angebotene Lernstoff. Was dies für den Lernertrag speziell des Vorlesungsprinzips bedeutet, wird im IV. Kapitel nachgeprüft.

\section{Empathische Beziehungen spielerischer Ungewissheit}

Auch ohne Neurobiologie ist schon lange klar, dass (Rollen-)Spiele geeignet sind, das Interesse von Schülerinnen und Schülern zu wecken und über die Ungewissheiten, die in Spielsituationen stecken, Lernerfolge zu erzielen. Praktiziert wird spielerisches Lernen vor allem in der Früherziehung. Kinder lernen erkennen, in welchen sozialen Beziehungen sie zueinander stehen und worin sie sich voneinander unterscheiden. Im Rollenspiel werden Möglichkeiten ausprobiert, etwas in unterschiedlicher Weise zu sagen und darzustellen. Dabei muss der Zusammenhang mit Grundfähigkeiten der Empathie berücksichtigt werden. Diese zeigen sich bei Kindern schon etwa im Alter von drei Jahren. In den Jahren danach lassen sie sich so entwickeln, dass in ihrem Lernapparat zum Teil komplexe Spielideen entstehen, die gemeinsam aufgebaut und umgesetzt werden können. ${ }^{17}$

Die Attraktivität dieses Lernansatzes zeigt sich auch in der Welt von Erwachsenen. An den Hochschulen können Rollenspiele helfen, sich in die Lebens- und Berufswelt anderer hineinzuversetzen. Besonders auffällig ist, dass sich Studierende der Sozialwissenschaften mit großer Begeisterung an spielerischen Simulationen internationaler beteiligen, die von den United Nations schon seit 1923 organisiert werden (,Manhattan-Model“). In der Vorbereitung der New Yorker Großveranstaltung, aber auch unabhängig von

Indessen können auch schmerzliche Erlebnisse fernstehender Menschen „,nahegehen“, da die neuronalen Strukturen affektiver und intellektueller Empathie miteinander vernetzt sind (Singer 2006).

17 Vgl. „Prinzipien zur Förderung metakognitiver und selbstregulativer Kompetenzen (4.4/d): 84, in: BMFT 2007 (Mitarbeit: Wassilius E. Fthenakis; Vorwort: Kristin Gisbert, Hans-Rainer Kunze, Renate Niesel, Corina Wustmann), Bonn-Berlin. 
dieser, übernehmen Studierende in der UNO-Generalversammlung, in ihren Ausschüssen und in verschiedenen UN-Unterorganisationen die Rolle nationaler Delegierter. Kolleginnen und Kollegen, die das Projekt im September 2011 betreuten, schwärmten von einer ,unglaublichen

Die Studierenden hätten das Projekt „zu einer eigenen Angelegenheit“ gemacht. Dabei liegt der Reiz dieses Lernformats offenbar darin, dass die im gespielten

(z. B. „Menschenrechte und Terror") füreinander eintretenden und gegeneinander kämpfenden Teilnehmenden füreinander nicht berechenbar sind und der Ausgang des Wettstreits jeweils offen ist. Ungewissheit und Rollenspiel fallen insoweit zusammen.

In der Sicht der lernorientierten Neurobiologie weckt die Ungewissheit, die in der Lernsituation liegt, Hoffnungen auf Belohnung. Die mit ihr verbundenen Lerneffekte hängen offenbar nicht nur vom absoluten Wert der Belohnung ab. Sie treten auch dann ein, wenn mehr Belohnung erfolgt als erwartet wurde (Spitzer 2007: 182). Neuronal getragen wird der belohnungsbezogene Lernvorgang von einem speziellen Gebiet des limbischen Systems (Basalganglien/Striatum) und dem Nucleus accumbens, in dem Dopamin erzeugt wird (Howard-Jones et al. 2011: 33). Wenn Schülerinnen und Schüler - in unterschiedlichen Forschungsdesigns - mit Lernaufgaben konfrontiert werden, die mit zufallsbedingten Ungewissheiten kombiniert sind (,learning games“ im Klassenzimmer), so wird belohnungstragendes Dopamin erzeugt. Das führt dazu, dass mit Verlusten im Spielverlauf jeweils die Hoffnung darauf verknüpft ist, dass die Gegner ihr Spielglück in der nächsten Spielphase verlieren (Howard-Jones et al. 2011: 34f.).

Auch Vorlesungen lassen sich als Rollenspiele verstehen. Im IV. Kapitel muss überlegt werden, inwieweit spielerische Ungewissheit im Rahmen dieses Lernformats lernfördernd wirksam sein kann.

\section{Lernen in Machtbeziehungen}

Da Medien für das, was öffentliche Meinung oder auch Öffentlichkeit genannt wird, immer bedeutsamer wurden, entstand seit den 1960er Jahren eine wachsende Aufmerksamkeit für die symbolische Seite gesellschaftlicher und politischer Prozesse..$^{18}$ Unter dem starken von Sozialpsychologie und Anthropologie gab insbesondere der amerikanische Sozialwissenschaftler Murray Edelman wichtige Anregungen für den Bereich der Politik. Er entwickelte ein

18 Vgl. Jürgen Habermas, Strukturwandel der Öffentlichkeit, Neuwied-Berlin 1962, eine Studie, die international rezipiert wurde. 
Instrumentarium, mit dessen Hilfe vor allem die expressiven Wirkungen untersucht werden können, die von Verdichtungssymbolen ausgehen: Symbole der Beschwichtigung führen auf der Ebene instrumenteller Politik zur Gefügigkeit, Symbole der Bedrohung lösen Erregung aus (Edelman 1976: Einleitung). Diese Anregungen lassen sich auch auf das hier interessierende Lernen an Hochschulen beziehen und mit dem Analyse-Ansatz des französischen Sozialphilosophen Michel Foucault verbinden.

\section{Instrumentelle und diskursive Macht im Lerndiskurs}

Foucault geht es um eine übergreifende Einbettung gesellschaftlicher Prozesse in allgemeine und spezielle Rahmenbedingungen, die er Diskurse nennt. Diese bestehen aus Symbolen und Ritualen, auch aus „materiellen Wirklichkeiten“ mit symbolischer Ausstrahlung (z. B. Architektur). Hinzu kommen einerseits wissenschaftliche Aussagen (Ansprüche auf Wissen und Wahrheit), die sich andererseits mit bestimmten Wertvorstellungen, Normen und Regeln mischen. Dabei versucht Foucault nachzuweisen, dass die Diskursebene auf die instrumentelle Ebene durchschlägt und auf die Akteure gesellschaftlicher Prozesse disziplinierend wirkt. Insoweit versteht er seine Diskurs-Analyse als Machtanalyse. Illustriert wird sie für unterschiedlichste gesellschaftliche Mikrobereiche (Klinik, Sexualität, Armee, Kirchen, Hochschule) und in seiner Studie Überwachen und Strafen speziell für das Gefängnissystem (Foucault 1977). ${ }^{19}$

In den Augen Foucaults sind weder marxistisch-ökonomische Zugriffe noch handlungstheoretische Ansätze geeignet, gesellschaftliche Machtverhältnisse als Beziehungen der Unterordnung von Subjekten angemessen zu betrachten. Foucaults aus Diskursen abgeleitetes Machtverständnis setzt sich speziell von einer Betrachtungsweise ab, die vor allem auf den deutschen Soziologen Max Weber zurückgeht (Wirtschaft und Gesellschaft, Erster Teil).

In Webers vielfach zitierter bedeutet Macht, ,jede Chance, innerhalb einer sozialen Beziehung den eigenen Willen auch gegen Widerstreben durchzusetzen, gleichviel worauf diese Chance beruht". Komplementär dazu hebt der im Hintergrund dieses Macht-Verständnisses stehende Herrschafts-Begriff auf die Chance ab, ,für einen Befehl bestimmten Inhalts Gehorsam zu Injedem Fall geht es um eine willens- und befehlsgeleitete Steuerung von Verhalten, gestützt auf die Drohung mit Sanktionen oder deren Einsatz. Diese Steuerungsmittel (=Ressourcen) werden bei einer solchen instrumentellen Betrachtungsweise ,gegen Widerstreben“ eingesetzt. Im Falle von Herrschaft verwandelt sich dieser Widerstand in Gehorsam. Dieser wie- 
derum wird im für Weber günstigsten Falle zur Disziplin: Sie gibt dem Machtund Herrschafts-Personal die Chance, „kraft eingeübter Einstellung für einen

Die Frage indessen, unter welchen Bedingungen sich solche befehlsunabhängigen Beziehungen von Macht und Herrschaft ergeben und wie sie mit disziplinarischer Wirkung „eingeübt“ werden, bleibt in der Perspektive Webers unbeantwortet. In diese Lücke stößt Foucaults Machtverständnis. Seine Antwort ist: Macht und Herrschaft sind diskursabhängig. Um Macht und Herrschaft zu fassen, müssen Macht- und Herrschafts-Diskurse untersucht werden.

Auf den Erziehungsbereich bezogen heißt das Folgendes: So unverzichtbar es sein mag, den Einsatz von Sanktionsmitteln und damit die Wirkungsweise instrumenteller Macht zu untersuchen, so notwendig ist eine Weitung des Blicks auf diskursiv-symbolische Rahmenbedingungen des Lernens. Analysiert werden muss der Lern-Diskurs, in den die Abläufe des Lernens - speziell auch im Hochschulbereich - eingefügt sind, da von ihm der Lernertrag abhängig ist.

\section{Zur Verschränkung diskursiver Macht mit der Handlungs- und Lernebene}

Auf welche Weise ist die Ebene diskursiver Macht mit der Handlungs- und der hier interessierenden Lern-Ebene verschränkt? In neurobiologischer Perspektive lässt sich dieses methodologische Problem überzeugender klären, als dies verschiedenen Diskurstheoretikern bislang gelungen ist.

Jürgen Habermas, der die schon erwähnten Forschungen zum Strukturwandel der Öffentlichkeit zu einer Theorie kommunikativen Handelns ausgeweitet hat, orientiert sich an Metaphern der Mechanik. Um die Arena ,deliberativer Demokratie“, ein spezielles Netzwerk diskursiver Macht (bei ihm: kommunikativer Macht), mit dem Entscheidungshandeln politischer Institutionen (Parlament/Regierung) und der sie tragenden Subjekte zu verbinden, bedient er sich eines „Schleusenkonzepts“. Der Gedanke, dass sich in Netzwerken verbreitete politikinhaltliche Vorstellungen in das Handeln von Akteuren „einschleusen“ lassen, ist empirisch jedoch nicht präzise genug, um die Verschränkung zwischen Diskurs- und Handlungsebene einsichtig zu machen.

Auch Foucault bearbeitet in seiner Machtanalyse „Modi der Implikation des Subjekts in die Diskurse“ (zit. nach: Eribon 1993: 192) mit Hilfe mechanisch-maschinenartiger Vergleichsbilder. Gefängnisse, Schulen und andere Lernbereiche sind für ihn Diskursmaschinen. Durch sie werden die Individuen diszipliniert, indem ihnen immer wieder neue Körperzustände aufgezwungen werden, die je speziellen Anforderungen entsprechen. (Foucault 1977: 173ff.). Dabei fehlt diesem von Foucault konzipierten maschinellen Übersetzungsme- 
chanismus der Bezug zu den Köpfen der Individuen - zu ihren neuronalen Netzwerken -, der im oben umschriebenen Konzept des „Embodied Learning“ erfasst wird (II.1.).

Was nunmehr den Lern-Diskurs angeht, so ist einerseits schon deutlich geworden, dass in ihm Komponenten stecken, die nach dem Muster automatischer Imitation übernommen werden. In Verbindung mit diesen tendenziell unbewusst wirksamen Lernimpulsen stecken im Lern-Diskurs andererseits Informationen, die auf Beobachtungen beruhen und tendenziell bewusst verarbeitet werden. ${ }^{20}$

Generell gilt, dass beliebige Interaktionen von der jeweiligen Informationslage abhängen (van Baaren u. a., in: Decety/Ickes 2011: 36-38). Speziell heißt das, dass hochschulische Lernbeziehungen in einen Lernkontext eingebettet sind. Aus ihm kommen die Informationen, die in die Beziehungen zwischen den Lernenden und den Lehrpersonen - auch in die Beziehungen der Lernenden untereinander - eindringen. Sie wirken auf das je bestehende Attachment, die auf ihm aufbauenden empathischen Verbindungen und auf Spiel-Beziehungen ein, soweit diese vorhanden sind.

\section{Zur Illustration diskursabhängiger Lernbeziehungen}

Am Beispiel einer einfach gelagerten Spiel-Konstellation kann versucht werden, den Mechanismus tendenziell bewussten Lernens aus Macht-Diskursen - hier Lerndiskursen - zu illustrieren. Zurückgegriffen wird auf eine im Experiment in vielen Varianten erprobte Versuchsanordnung, in der es einerseits spielende Kinder und andererseits Kinder gibt, die Spiel-Szenen beobachten („False-Belief-Test“; Verplaetse 2011: 40). Dabei stört es nicht, dass die beobachtenden Kinder die Spiel-Szenen nicht direkt erleben, sondern als Geschichte von spielenden Kindern vorgelesen bekommen, da dies, was die neurobiologische Verarbeitung des Spielgeschehens angeht, keinen Unterschied macht. Auch ist es für die Zwecke der Illustration unerheblich, dass die Versuchsanordnung in entwicklungspsychologischer Perspektive gewählt wurde, um herauszubringen, ab welchem Alter heranwachsende Kinder über die Fähigkeiten zur Empathie verfügen. Derselbe Empathie-Test lässt sich analog auch auf Erwachsene beziehen, um zu ermitteln, ob ihre empathischen Fähigkeiten intakt sind. Die Versuchsanordnung, in der die beobachtenden Kinder zu den - im vorgelesenen Text - spielenden Kindern in Lernbeziehun-

20 Hier kann an die auf Beobachtungslernen gerichtete Imitationstheorie von Albert Bandura und Richard H. Walters angeknüpft werden, der es indessen an neurobiologischen Bezügen fehlt (Vgl. Mazur 2004: bes. Abschnitt 12.1.4). 
gen stehen, die von einem Lernkontext abhängig sind, gestaltet sich folgendermaßen:

Die Aufmerksamkeit der beobachtenden Kinder wird in der Geschichte auf zwei spielende Kinder gelenkt, die sich mit Murmeln vergnügen. Eines dieser Kinder versteckt eine Murmel in einem Korb - hier Behältnis 1 genannt - und verlässt das Spielzimmer. Das im Raum verbleibende Kind nimmt die Murmel aus dem Korb und versteckt sie in einer Schachtel - hier Behältnis 2 genannt. Als das Kind, welches das Zimmer verlassen hat, zurückkommt, möchte es die versteckte Murmel haben.

Die mit Hilfe dieser Geschichte getesteten beobachtenden Kinder werden danach gefragt, wo das in das Spielzimmer zurückgekommene Kind die Murmel suchen wird. Sie bestehen den Empathie-Test, wenn sie annehmen, dass im Behältnis 1 gesucht wird. Auf diese Weise zeigen sie, dass sie dank intellektuell-mentalem Nachvollzug wissen, dass das von draußen kommende Kind die Veränderung des Verstecks nicht mitbekommen hat und daher von ihm keine Nachforschungen im Behältnis 2 erwartet werden können.

Dieser Test und sein Ergebnis - ab ungefähr drei Jahren bestehen ihn Kinder - passen zu Damasios schon erwähnter neurobiologischen Durchdringung des Empathie-Problems. Er arbeitet die neuronal abgestützte Fähigkeit von Kommunikationspartnerinnen und -partnern - im Testbeispiel sind es die beobachtenden Kinder - heraus, das für die jeweilige Partnerin und den jeweiligen Partner bestehende Problem - im Testbeispiel das Problem des ins Zimmer zurückgekommenen Kindes - so zu simulieren, als sei es das eigene (Damasio 2010: 113-116).

Überträgt man diese Test-Konstellation auf die empathischen Lern-Beziehungen an Hochschulen, so ist die Informationslage des Lernkontexts für die Testpersonen zwar viel komplexer als für die beobachtenden Kinder. Einfachen Informationen über Versteckspiele steht eine Fülle von Informationen gegenüber, die Lernende und Lehrpersonen aus dem Lern-Kontext beziehen. Analog geht es darum, sich mit Hilfe von Informationen in die Lage jeweiliger Lernpartnerinnen und -partner hineinzuversetzen. Ebenso wie die Beobachter-Kinder aus den Versteck-Informationen Schlüsse auf die kognitive Lage der beobachteten Kinder ziehen, können Lernende - und umgekehrt auch diejenigen, die das Lernen anleiten, -mit Hilfe der für sie im Lern-Kontext verfügbaren Informationen nachvollziehen, was sich im Denken der Lernpartnerinnen und -partner abspielt. Man kann sich dies folgendermaßen vorstellen:

Im Lerndiskurs werden Spielideen verbreitet, u. a. Ideen zum Murmelspiel. Sie werden von Kindern, vermittelt über andere Kinder oder auch über Erwachsene, aufgenommen und im Spiel umgesetzt. Lernende, die dem Spiel als Beobachtende folgen oder Berichte und Geschichten über Spielabläufe hören, lernen dadurch, sich in die Situation der Spielenden hineinzuversetzen. Dieser 
Nachvollzug hat etwas Imitatives, wenngleich nicht unbedingt Zwingendes und Disziplinierendes. Ist es doch ohne Weiteres möglich, dass empathisch am Murmelspiel beteiligte Beobachtende und Lernende die Erwartung entwickeln, dass das ins Zimmer zurückkommende Kind, nachdem es die Murmel im untersuchten Korb nicht gefunden hat, sein Such-Glück in Behältnis 2 überprüft.

Demselben Mechanismus folgend darf man sich über informierte Empathie laufendes Lernen aus dem Lern-Diskurs auch an Hochschulen vorstellen. Der Unterschied ist nur, dass der Lern-Diskurs, in dessen Rahmen gelernt wird, sehr viel komplexer ist, als durch die einfachen Regeln des Versteckens von Murmeln an einem einfachen Beispiel angedeutet wird. 


\section{Allgemeine Lern-Konstellation}

Von der Journalistin Christiane Florin, einer langjährigen Lehrbeauftragten am Institut für Politikwissenschaft der Universität Bonn, stammt ein Essay zum Lernen an Hochschulen, der auf eine „Studierenden-Beschimpfung“ hinausläuft. Die Autorin verdichtet ihre harsche Kritik an den Studierenden zu dem Vorwurf, diese hätten sich kraftlos an das „System“ angepasst (Titel: „Warum unsere Studenten so angepasst sind“" 2014).

Florin bemängelt, dass ihr die unerwünschte Aufgabe zufalle, als Lehrbeauftragte die Studierenden animieren, motivieren und orientieren zu müssen (Florin 2014: 67). In Erinnerung an ihre eigene Studienzeit in den 1980er Jahren empört das die Autorin, weil sie selbst und ihre Mit-Studierenden viele Fragen sowie Streit- und Diskussionslust mitgebracht hätten (ebd.: 65), was sie bei ihren Studierenden vermisst. Es fehle ihnen das Bedürfnis (bei Florin die „Sehnsucht“, ebd.: 9), „das Freiheitsversprechen“ auf Bildung einzulösen. Dies ist ein von der Autorin mehrfach formulierter (Selbst-)Anspruch, dem Studierende nach ihrer Auffassung gerecht werden müssen (ebd.: 78). Stattdessen stößt sie auf „Desinteresse“ (ebd.: 75) und darauf, dass einseitig auf die Anwendbarkeit des Studiums geschaut werde („Employability“, ebd.: 79). Mit besonderem Unverständnis registriert die Autorin verschiedentlich, dass die Studierenden die Formalien, das „Verschulte“ des Studiums, schätzen: „Vorgefertigte Stundenpläne und detaillierte Arbeitsanweisungen mindern aus ihrer Sicht das Risiko, Fehler zu machen." (ebd.: 52)

Diese weit verbreitete Betrachtungsweise unterstellt fachliche Kompetenz und unermüdlichen Einsatz der Lehrpersonen einerseits, mangelnde Eignung und fehlendes Engagement der Studierenden andererseits. Indessen fehlt es an einer Berücksichtigung der - von den Lehrpersonen maßgeblich mitbestimmten - Rahmenbedingungen, unter denen studiert wird.

Dem wird hier eine allgemeine Lernkonstellation entgegengestellt, die von diesen Bedingungen ausgeht. Im Folgenden werden diese in einem ersten Abschnitt (1.) als Machtdiskurs (= Lerndiskurs) in ihren wichtigsten Komponenten dargestellt. Durch ihn wird bei den Studierenden der Eindruck erweckt, dass sie an ihrer Hochschule nur "Winzlinge“ sind. Gespeist wird diese Botschaft der Winzigkeit einerseits von den Ritualen, die den Lernbetrieb begleiten. Andererseits ist es das im Diskurs steckende Prinzip des leistungsbezogenen Lernens nach Noten, das die Lernenden klein macht. Zugleich sind die Lernenden speziellen Diskurs-Botschaften der Winzigkeit ausgesetzt. Diesen begegnen sie besonders beim Besuch von Sprechstunden und sie entstehen auch dadurch, dass die Hausarbeiten der Studierenden zu wenig gewürdigt werden. 
In einem zweiten Abschnitt (2.) wird dann in neurobiologischer Perspektive deutlich, was die in ihrem Lernapparat verarbeiteten Eindrücke der Winzigkeit für die Studierenden bedeuten und zu welchen Einschätzungen der allgemeinen Lern-Konstellation sie kommen. Dabei lässt sich schwer entscheiden, bis zu welchem Grade diese Bewertungen bewusst oder unbewusst erfolgen. In der Verarbeitung der Diskurs-Botschaften ergeben sich Unterschiede zwischen einer großen studentischen Mehrheit und einer privilegierten Minderheit. Zu Letzterer gehören Promovierende, deren Plagiatspraxis in den letzten Jahren breit diskutiert wurde.

\section{Lernende im Macht-Diskurs (=Lern-Diskurs)}

Es ist nicht zu bezweifeln, dass sich die Traditionshochschulen und erst recht die Neugründungen immer stärker dem Strukturmodell marktorientierter Unternehmen annähern. An die Stelle staatlicher Lenkungsbehörden treten innerorganisatorische Apparate. Gerade diese Entwicklungen zur kalkuliert bürokratisierten und auch kommerzialisierten Lernwelt der Hochschulen gehen aber höchstwahrscheinlich an allgemeinen menschlichen Bedürfnissen nach emotionaler Einbindung vorbei. Für die Befriedigung solcher Bedürfnisse ist es wichtig, dass sie im Umfeld besonders der erneuerten alten Universitäten und teilweise auch im Design der Neugründungen symbolische Anknüpfungs-

\section{Rituale}

Oft wird an Hochschulen studiert, die immer noch in „historischen Gebäuden“ untergebracht sind. Lernende tauchen in eine altehrwürdige Institution ein, sitzen in Bibliotheken, in denen riesige Bücherwände und Holzverkleidungen eine Feierlichkeit ausstrahlen, die an das Ambiente von Gotteshäusern, Gerichtsgebäuden und Verhandlungsräumen erinnert. Der Hauch von Würde, der insbesondere in den Hörsälen weht, gehört auch zum Design neugebauter Hochschulen. Selbst wenn die hier ausgestrahlte ,neue Ehrwürdigkeit" eher kalt ist, haben die Studierenden das Gefühl, in eine besondere Institution eingelassen zu werden. ${ }^{21}$

So sehr die technisch-medialen Rahmenbedingungen des Lernens verändert sein mögen, so unverkennbar ist, dass sich die Tages-, Wochen- und

21 Vgl. zur neurobiologischen Erklärung des „Sich-klein-Vorkommens“ in großen Räumen: Birbaumer 2014: 412 und Kap. 27. 
Semesterrhythmen des Lernbetriebs erhalten haben. Die Studierenden erleben den Wechsel von einem Semester zum anderen wie ein Naturereignis, vergleichbar dem Wechsel der Jahreszeiten. Wenn zwischen „Vorlesungszeit" und „Vorlesungsfreier“ Zeit unterschieden wird, so verweist das auf Vorlesungen als zentrales Lernformat. In einer langen Geschichte sind sie zum ,rituell-symbolischen Glanzstück" des gesamten Hochschulbetriebs geworden und erwecken den Anschein ertragreicher Wissensvermittlung. ${ }^{22}$

Am Anfang des Semesters sind die Kräfte jeweils angespannt. Es folgt eine eher ruhige Zwischenphase, die dann in einen anstrengenden Endspurt übergeht. ,Antizyklische“ Wünsche und Aktivitäten werden in diesem regelmäßigen Auf und $\mathrm{Ab}$ überspielt.

Das symbolische Make-up des Universitätsalltags schließt auch den eingespielten Sprechstundenbetrieb ein, der in der Regel zeitlich sorgfältig reglementiert ist. Das Layout von Türschildern, Briefköpfen und Stempeln kommt dazu. Auffällig ist auch eine geheimnistuerische Gutachtenpraxis, ein so gut wie unausrottbares Beharren auf akademischen Titeln und auf der verschnörkelten Anrede von Funktionsträgern (z. B. und „Spektabilität").

Zum tiefergehenden Wurzelwerk solcher symbolischen Attribute des Lernbetriebs gehört ein nie völlig aufgegebenes Familien-Leitbild der deutschen Hochschulen. Das Sprachbild der „Alma Mater“, die ihre Kinder ernährt, ist bis heute ebenso wenig angetastet wie die Figur des Doktorvaters, neuerdings auch diejenige der Doktormutter. Väter und Mütter sorgen für die ihnen anvertrauten Schülerinnen und Schüler, die stolz auf ihre je besondere akademische Abstammung verweisen können. Auch die Humboldt'sche Formel von der „Gemeinsamkeit von Lehrenden und Lernenden“ ist immer noch im Umlauf. Zudem hält sich weiterhin eine Vorstellung von ,heiliger Wissenschaft"“23, die auch außerhalb der Hochschulen verbreitet ist. Man braucht nur darauf zu achten, wie andächtig studentische Anfängerinnen und Anfänger das Wort

22 Gegen diesen,,äußeren Schein“versucht das IV. Kapitel in neurobiologischer Perspektive zu argumentieren. Unterstützt wird diese Argumentation durch Überlegungen des Bielefelder Hochschullehrers Jörn Loviscach, für den ,die klassische Großvorlesung ein offensichtlich zum Scheitern verurteilter Versuch zu sein (scheint), Wissen zu vermitteln“. Loviscach vergleicht die Großvorlesung mit ihrer digitalisierten Version, die er selbst praktiziert, weil er den Verdacht hat, dass diese symbolisch-rituell Erwartungen weckt, die sie nicht einlösen kann (Loviscach 2016: 13).

23 Von Wissenschaft als einem „Heiligtum“ sprach beispielsweise Hedwig Dohm, eine der mutigen Frauen der zweiten Hälfte des 20. Jahrhunderts, als sie ihre Ansprüche an die universitäre Männergesellschaft anmeldete. Vgl. den Hinweis bei Ferdinand Menne: Lässt die ,Alma Mater' ihre Töchter nicht zu sich kommen? in: Neue Gesellschaft/ Frankfurter Hefte, 34. Jg., Juli 1987 (H.7): 635. 
Wissenschaft in den Mund nehmen und nach Geheimnissen fragen, deren Entschlüsselung sie sich wünschen.

Deutlich wird hier, dass es eine Kontinuität jener speziellen symbolischen Elemente der Universitätstradition gibt, die von der Studentenbewegung der zweiten Hälfte der 1960er Jahre in Frage gestellt wurde. Zu erinnern ist an den belächelten und karikierten „Muff der tausend Jahre“, von dem moniert wurde, dass die „Lehrenden“ ihn unter den „Talaren“ konservierten. Das Scheitern der radikalen Reformbestrebungen der 1968er und die Bekräftigung der hierarchischen Prinzipien der sogenannten Ordinarienuniversität im Hochschulrahmengesetz des Jahres 1974 hatten Folgen für die dekorativen Bestandteile der Universitätstradition. Es war wieder Platz nicht nur für Talare, Barett und Handschuhe bei feierlichen Immatrikulationen, Examens- und Promotionsfeiern. Zugleich wurden alle nie aufgegebenen Restbestände der altehrwürdigen Institution Universität aufgewertet. Nimmt man alle absichtsvoll demonstrierten oder sich absichtslos entfaltenden Elemente universitärer Ritualität zusammen, so lassen sie sich als Mythen verstehen, die aus der Vergangenheit in die Gegenwart hineinreichen.

\section{Lernen nach Noten}

In Alltagsgesprächen von Familien, in denen höhere Schulbildung und Berufsbildung zur Tradition gehören, gibt es eine Redewendung, die sich gleichermaßen auf die Lernhaltung von Schülerinnen und Schülern sowie Studentinnen und Studenten beziehen lässt. In einer beschreibenden Variante bringt sie zum Ausdruck, dass $\mathrm{X}$ begriffen habe, worum es gehe. In einer fordernden Variante wird von Y gesagt, er oder sie müsse (endlich) begreifen, worum es gehe. Gemeint ist Leistungsbereitschaft. In der Sprache sowohl von Schulen als auch von Hochschulen ist es die Bereitschaft, ,etwas zu tun“. Anhand von Prüfungen und Noten wird erkennbar, ob die bestehenden Erwartungen erfüllt werden. Um ,gute Noten“ geht es auch schon bei Leistungsnachweisen, die im Vorfeld von Prüfungen erbracht werden müssen.

Es kann hier keine Geschichte der inneruniversitären Entfaltung des notenbezogenen Leistungsgedankens geschrieben werden. Da die Hochschulen historisch zunächst vor allem für privilegierte Schichten und nur für wenige Außenseiter zugänglichen waren, brauchten sie keine ausgefeilten Notensysteme. Die gesellschaftliche und

Stellung der Absolventen war prinzipiell nicht vom Universitätsabschluss abhängig. Dennoch entstand die aktueller studentischer Milieus nicht erst in Zeiten hochgradiger Arbeitsplatzunsicherheit. Schon in der Phase der Studentenbewegung, als sich - trotz erster ökonomischer Rezession (1966/67) - niemand um einen zufriedenstellenden Arbeitsplatz sorgen musste, kam es nie zu einer prinzi- 
piellen Aufkündigung des Leistungsgedankens und des mit ihm verbundenen Lernens nach Noten. Die meisten Studierenden scheuten keine Anstrengung, auch neben ihrer sogenannten politischen Arbeit die verlangten

zu erwerben. Soweit die Arbeitskraft einiger Aktivisten vorübergehend aufgesaugt worden war, wurde das Versäumte nachgeholt. Mit großer Energie brachten viele 1968er - Rudi Dutschke eingeschlossen - ihre Examina und Promotionen unter Dach und Fach. Nach zeitweiligen Irritationen setzten sich daher die Hochschulen als Leistungs- und Prüfungsräume durch. Die fortdauernde Bereitschaft zur Leistung wird auch durch Ergebnisse der sogenannten Jugendforschung belegt. ${ }^{24}$

Der Heißhunger nach Leistung und Noten hat sich in Vier-Augen-Gesprächen mit Studierenden der Sozialwissenschaften immer wieder aufs Neue bestätigt. Wenn Sprechstunden dazu dienen sollen, das Ordnen und Gliedern von Hausarbeiten zu diskutieren, die Logik der Gedankenführung zu überdenken sowie Darstellungs- und Zitier-Techniken zu überprüfen, ist die Notenfrage unterschwellig immer präsent. Der Wunsch, das Notenurteil zu erfahren, ist oft so dringlich, dass die Durchsicht von Stärken und Schwächen der Arbeit oft gar nicht ,notenfrei“ beginnen kann. Gelingt es trotzdem, so kommt es über kurz oder lang zum abrupten Gesprächsstopp mit der Frage: „Wie schätzen Sie mich ein?" Abwehrende Signale werden durch bangende und hoffende Blicke aufgefangen. Spätestens am Schluss solcher Gespräche, in denen sich Studierende in quälender Weise hingehalten fühlen, gibt es dann kein Halten mehr: „Jetzt möchte ich aber doch wissen, was für eine Note ...!"“

Durch solche Erfahrungen, aufbauend auf der eigenen Sozialisation in Schulen und Hochschulen, sind auch diejenigen, die das Lernen anleiten, auf Noten Im hochschulischen Diskurs sind Noten ein Medium der Reglementierung, das an den „klinischen Blick“ (Foucault 1976) erinnert, der das Verhältnis zwischen Ärztinnen und Ärzten sowie Patientinnen und Patienten im Kliniksystem bestimmt. Durch ihn schrumpfen kranke Menschen auf ihre gestörten Organe und Funktionen zusammen. Dem entspricht, dass Studierende für mündliche und schriftliche Leistungen stehen, die an einer traditionellen oder neuerdings auch veränderten Notenskala abgelesen werden können. Im ungünstigsten Fall verengt sich der Notenblick zusätzlich noch dadurch, dass er ausschließlich auf schriftlich fassbare Leistungen gerichtet ist und sich dabei nicht hauptsächlich auf Inhalte bezieht, sondern Kriterien formaler Korrektheit (Stil, Grammatik, Rechtschreibung, Zitierweise) bevorzugt.

24 Vgl. für das Ende der 1970er bis Mitte der 1980er Jahre: Geißler 1985: 27-37. Für die neuere Entwicklung ist auffällig, dass die Leistungsbejahung mit materiellen und hedonistischen Ansprüchen kombiniert wird. Vgl. Shell Deutschland Holding (Hrsg.) 2010: 196ff. 
Seit die Zukunft der Arbeit unsicher ist, haben sich die Fixierung auf Noten und der Notenblick zweifellos noch verschärft. „Gute Noten“ gelten als wichtige, vielleicht entscheidende Voraussetzung dafür, eine „gute Stelle“ zu Indirekt lässt sich der über Noten vermittelte Zusammenhang zwischen universitärer Gegenwart und Zukunft den Berichten und Klagen von Arbeitslosen entnehmen, die auf ihr Studium zurückblicken. Illustriert durch Tagebuchauszüge eines ausgebildeten Gymnasiallehrers, lässt sich folgende Grundlinie der Argumentation erkennen ${ }^{25}$ :

„Ich habe mich sehr angestrengt und gute Leistungen erzielt. („Meine Examensnoten liegen bei gut $(1,63)$ und sehr gut $(1,50)$, bei einer Gesamtnote von 2,15. Ein durchaus beachtliches Ergebnis, möchte man meinen, rechnet man, dass dafür immerhin dreißig (!) mündliche und schriftliche Prüfungen absolviert werden mußten. Trotzdem werde ich arbeitslos sein.") Mit dem Aufzählen von Noten und Notendurchschnitten verbinden sich ungläubig bis anklagend vorgetragene Belege dafür, dass sich die erworbenen nen nicht in eine sinnvolle und der Ausbildung angemessene Arbeit umsetzen lassen. („Langsam fühle ich mich als ausgebildeter Gymnasiallehrer wie ein Mensch zweiter Klasse. Sind denn siebeneinhalb Jahre Anstrengung mit Referendariat und eine seltene Dreifächerverbindung nichts wert in diesem Staat?")

In solchen Anklagen artikuliert sich auch das herkömmliche Verständnis von Noten als Sanktionsmittel. Studierende, die keinen angemessenen Arbeitsplatz fühlen sich ungerecht behandelt. Aus ihrer Sicht haben sie sich dank ihrer Leistungen, die sich in ,guten Noten“ ausdrückt, nicht Strafe, sondern Belohnung verdient.

In den neuen Bachelor-Studiengängen führt der Notenblick zu einer besonders engmaschigen Version Lernens. Leistungen werden im Rahmen von Modulen benotet und nach dem sogenannten ECTS-System in Credit Points abgerechnet. Dabei wird die im herkömmlichen Notensystem angelegte Präzisionssymbolik weitergeführt. Der durch Stellen hinter dem Komma vermittelte Eindruck „absoluter Notengerechtigkeit“ wird dadurch bekräftigt und noch verstärkt, dass schwer messbare Spezialleistungen in differenzierte Notenskalen gepresst werden.

25 Peter Dempf, Das hundertfache Nein. Tagebuch eines frisch ausgebildeten Gymnasiallehrers, der ein Unterkommen sucht, in: Süddeutsche Zeitung, 27./28.8.1988. 


\section{Warten auf die Professorin und den Professor}

Die wohl drastischste Beschreibung des Diskurses, der die alltäglichen Lernbeziehungen bestimmt, ist die einer „Art Herrgottsverhältnis“ (zit. nach: Krüger u. a., 1986: 298). Die Erlebnisse, auf die hier Bezug genommen wird, schildert eine Leserzuschrift an die Frankfurter Allgemeine Zeitung folgendermaßen: „Da geht man nun als Student mit dem festen Vorsatz, in diesem Seminar wirklich von Anfang an mitzuarbeiten, zum ersten angekündigten Seminartermin und kann dann wenig später wieder den Heimweg antreten: Da lässt der Professor durch seine Sekretärin mitteilen, dass er erst nächste Woche kommen kann, man erfährt von einem kleinen Zettel, dass er die Veranstaltung wegen anderweitiger $\mathrm{V}$ ganz absagen muss, oder man wartet eine halbe Stunde, und selbst ein Anruf bei Professors zuhause bringt kein Ergebnis.“ (FAZ, 21.10.1982, zit. nach: Gleich u. a., 1982: 72f.)

Erfahrungen der Geringschätzung machen Studierenden vor allem im Umfeld von Sprechstunden, die in den formell angekündigten Zeiten als lächerlich kurz empfunden werden: „Sprechstunde von Prof. X: Mo 11.00 bis 12.00 Uhr". Das ist die stillschweigende Aufforderung, sich auf jeden Fall kurz zu fassen und sich überhaupt genau zu überlegen, ob ein Besuch überhaupt nötig ist. Oft werden Sprechstunden auch kommentarlos gestrichen.

Ein Studierender fasst die Erfahrungen, die er am Otto-Suhr-Institut der FU Berlin Anfang der 1990er Jahre machte, folgendermaßen zusammen: „Wenn ich hier zu einem Prof. gehe, habe ich immer das Gefühl: ,O Gott, noch ein Student, o Gott, was will der jetzt von mir? Möglichst ... ich bin eigentlich gar nicht da, ich habe einen Termin, machen Sie bitte kurz. Sie wollen Ihre Arbeit besprechen; ja, war sehr schön, sehr schön, ja 1,7`. Davon habe ich nichts, da interessiert sich keiner." ${ }^{\text {"26 }}$

20 Jahre später hat eine Studentin der Asienwissenschaft, die als freie Journalistin auf ihre Studienzeit zurückblickt, ähnliche Erinnerungen. Einer ihrer Professoren, so schreibt sie, „kam tendenziell nie“. Die Sekretärin hielt ihn „im Moment für sehr beschäftigt". Das Warten, so fügt die Autorin hinzu, „hätte zum Lernen einer weiteren Fremdsprache gereicht“" (Forschung\&Lehre 11/2014).

$\mathrm{Zu}$ den Erfahrungen der Winzigkeit, die in Gesprächen mit Studierenden erwähnt werden, gehört auch, dass es am angemessenen Feedback auf die mühsam und mit großem Zeitaufwand geschriebenen Hausarbeiten fehlt.

26 Dies sind Auszüge aus einem von 10 qualitativen Interviews, die von Angelika Koch, Diplomandin des Otto-Suhr-Instituts der FU Berlin, im Rahmen ihrer Diplomarbeit 1993 geführt wurden. (Zit. nach: Frankfurter Rundschau, 30.12.1993: „O Gott, noch ein Student, was will der jetzt von mir?“). 
„Produkte meiner wochenlangen Arbeit“, so erlebt es eine Studentin, seien „im Schrank meines Dozenten verschwunden“ und damit ,in der Bedeutungslosigkeit versunken“ (Ladenthin 2016: 43f.; auch 117).

Einen „Winzigkeitsbericht“, der sich über mehrere Etappen erstreckt, stammt von einer Studentin der Politikwissenschaft. Sie berichtet, dass ein „,vielbeschäftigter Professor“ ihre Hausarbeit überhaupt nicht zur Kenntnis genommen hat und ihr so der angestrebte Leistungsnachweis fehlte. ${ }^{27}$ Es geht um ein Proseminar zur „Europäischen Integration“ im Sommersemester 1989. Da zu einem Stichtag die Hausarbeit abgeliefert werden musste, machte sich die Studentin schnell ans Werk:

„Ein heißes Maiwochenende wird durchgetippt; pünktlich liegt die Hausarbeit auf dem Tisch des Seminarleiters.“ Die Studentin fährt in die Ferien, das anregende Seminar überall weiter und will den für Mitte August angekündigten Schein bei der Sekretärin abholen: „Ende August: Die Scheine sind immer noch nicht da-naja, Herr Dr. X soll auch mal Urlaub machen."

Weitere vier Wochen vergehen. Nun möchte die Studentin endlich die Bestätigung für die im letzten Semester geleistete Arbeit, um dementsprechend motiviert das nächste zu beginnen. Aber wieder begegnet ihr die Sekretärin im Geschäftszimmer nur mit einem Kopfschütteln. Genauso Anfang, Mitte und Ende Oktober. Einige aus dem Seminar haben den Schein schon!

Die Sekretärin von Dr. X erteilt die Auskunft, er sich in sehr arbeitsintensiven Vorbereitungen für einen Kongress. „, Man hat Nachsicht-wie unwichtig einem die eigene Hausarbeit in Anbetracht des Stresses eines Dozenten doch vorkommen kann. "

Schließlich und endlich kommt die Nachricht, Dr. X habe alle Arbeiten längst weggeworfen -,,ALTPAPIER, MEINE HAUSARBEIT Pech c'est la vie ...

Also nochmal die Arbeit kopieren ... abgeben, die Entschuldigungen des Dozenten über sich ergehen lassen (man kann ihm gar nicht so richtig böse sein) und warten. Wie lange diesmal, Dr. X? “

Auffällig an all diesen Erlebnissen des zum Teil vergeblichen „Wartens auf die Professorin und den Professor" ist, dass die unzumutbar behandelten Studierenden sich zwar sehr ärgern, aber doch letztlich bereit sind, die Kröten zu schlucken, die ihnen zugemutet werden. Diese - in anderer Terminologie - Schere in ihrem Kopf bedarf der Interpretation in neurobiologischer Perspektive.

27 Bettina Klee, Auf der Suche nach der verlorenen Hausarbeit. Eine Beschwerde, in: Politicum (Fachschaft des Bonner Seminars für Politikwissenschaft) WS 1989/90 (Nr.1): 7; kursive Hervorhebungen durch den Verf. 


\section{Zur Neurobiologie der Winzigkeit}

Von Ausnahme-Phasen der Mobilisierung studentischen Protests abgesehen, sich unter den Studierenden nur einige Wenige, die aufgrund ihrer speziellen Voraussetzungen dagegen protestieren, dass ihnen Winzigkeit abverlangt wird. Sie kommen in der Regel aus bildungsfernen Schichten, haben zumeist einen schwierigen Bildungsweg hinter sich und erwarten von der Hochschule, dass sie ihren Heißhunger nach Bildung zu stillen bereit ist. ${ }^{28} \mathrm{Zu}$ diesen Einzelnen gehören auch Studierende, die mit Berufserfahrung an die Hochschule gehen und hohe Erwartungen an ihre W zierung mitbringen. Für alle anderen Studierenden lässt sich in neurobiologischer Sicht erkennen, dass sie sich ruhigstellen lassen. Speziell gilt das unter dem Gesichtspunkt mangelnder Hochschuldidaktik und unter dem Gesichtspunkt mangelnder Betreuung und Beratung.

Neben der großen Mehrheit der Studierenden lässt sich eine kleine Minderheit - abzüglich der genannten Einzelnen - ausmachen, die privilegiert ist. Sie wiederum ist bereit, sich ruhig stellen zu lassen, weil sie sich ihre Privilegien nicht verscherzen will.

\section{Allgemeine Ruhigstellung einer großen studentischen Mehrheit}

Für unterschiedliche Anwendungsfelder konnte der erwähnte Murray Edelman plausibel machen, dass symbolische Politik dazu eingesetzt werden kann, politische Ziele zu erreichen („Use of symbolic politics“). Beispielsweise leuchtet ein, dass Wahlen und speziell die stark ritualisierten zwischenparteilichen und Wahlkämpfe bei den beteiligten Bürgern den Eindruck erwecken, als könnten sie auf politische Entscheidungen einwirken. Die über symbolische Elemente des Wahlkampfs vermittelte Folge ist, dass die Bürger dazu bereit sind, sich solchen Entscheidungen zu fügen und darüber hinaus Gefühle der Verbundenheit mit einem politischen System zu entwickeln, das ihnen die Beteiligung an Wahlen ermöglicht (Edelman 1976: 15; 84f.).

$28 \mathrm{Zu}$ dieser studentischen Spezies gehörte ein von mir befragter Student der Politiksowie Geschichtswissenschaft und Volkswirtschaft, den die schmerzliche Erfahrung einer dauernden Abweisung und Unterforderung dazu brachte, Vorschläge zu einem elitistisch gefärbten Ausbildungssystem zu entwickeln: Einer Grundausbildungsphase für alle Studierenden sollte eine „2jährige Spezialisierungsphase der studentischen Mehrheit und danach eine mehrjährige Ausbildungsphase folgen, die einer wissenschaftlichen Elite vorbehalten sein sollte". In diese Elite wollte ein Student aufgenommen werden, der seine Unzufriedenheit in ein elitistisches Modell umsetzte, vor dem er - von seiner Grundposition her anti-elitistisch orientiert - dann selbst zurückschreckte. 
Bezieht man Edelmans doppelte Perspektive der Beschwichtigung/Fügsamkeit einerseits und der Bedrohung/Erregung andererseits auf die neuronalen Mechanismen der Reizverarbeitung, so werden Erkenntnisse der Neurobiologie angesprochen, die sich auf die Wirkungsweise sogenannter Neuromodulatoren beziehen. Am Wahlkampfbeispiel ist einerseits klar, dass das leidenschaftliche Engagement in der zwischenparteilichen Auseinandersetzung, soweit Bürgerinnen und Bürger aktiv teilnehmen, zur Produktion des Neuromodulators Dopamin anregen kann. Soweit dieses Dopamin die für Wahlkampfreize zuständigen neuronalen Netze in Zustände der Erregung versetzt, kann es dazu kommen, dass fanatisierte, im schlimmsten Fall aggressiv-gewaltorientierte Aktivisten sich um die Vergabe von Parlamentssitzen streiten. Dem widersprechen vielfältige Beobachtungen, die für westliche Demokratien der Gegenwart belegen, dass Edelman Recht hat, wenn er erwartet, dass an Wahlkämpfen beteiligte Bürgerinnen und Bürger beschwichtigt und fügsam gemacht werden. Neurobiologisch heißt das, dass nicht oder doch nur am Rande der Neuromodulator Dopamin wirksam ist. Stattdessen spielt mit hoher Wahrscheinlichkeit der Neuromodulator Serotonin eine Rolle. Folgt man den Ergebnissen der Forschung über seine Wirkungsweise, so wird er im Rückenmark in den sogenannten Raphe-Kernen der retikulären Formation produziert. In den zahlreichen Zielgebieten, die er erreichen und in denen er aufgenommen werden kann, kommt es zu einer Dämpfung und Verhinderung impulsiver Handlungen. ${ }^{29}$ Das passt zu Edelmans These, dass Wähler durch symbolische Wahlkampfpolitik ruhiggestellt werden.

Gelten solche Beobachtungen und Überlegungen analog auch für die skizzierte hochschulische Ritualität, so lässt sich das Folgende annehmen: Wie unzufrieden, zum dopamin-gestützten Protest neigend, Studierende auch sein mögen, so dominiert im Lernbetrieb doch ihr rituell erzeugtes, serotonin-gesteuertes Einverständnis mit einer Lern-Konstellation, in der sie auf stillschweigendes Mitmachen festgelegt werden.

Überprüft man in entsprechender Weise die Fixierung auf Noten, so kommt es auch über dieses reglementierende Medium des hochschulischen Diskurses dazu, dass Studierende beschwichtigt werden. Neurobiologisch heißt das Folgendes: Soweit sich gegen Missstände der allgemeinen Lern-Konstellation dopamin-gestützte Widerstände zeigen, die u. a. durch eine vielfach erwiesene und belegte Willkür der Notengebung inspiriert sein können, so wirkt dem die Produktion von Serotonin entgegen. Indem dieser Neuromodulator auf zahlram 2010: 32). 
reiche Zielgebiete, im Besonderen auf Abschnitte des sogenannten limbischen Systems dämpfend einwirkt, werden Studierende ruhiggestellt.

Unter dem Gesichtspunkt des herkömmlichen Verständnisses von Noten als Sanktionsmittel erfolgt daher die Reizverarbeitung, für die die Basalganglien (Striatum) und Nucleus accumbens zuständig sind, im Zeichen serotonin-gesteuerter Dämpfungseffekte. Unter solchen Umständen wirkt das reglementierende Medium des Notenblicks darauf hin, dass schlechte Noten von den Betroffenen auch dann hingenommen werden, wenn sie in ihren Augen nicht leistungsgerecht sind.

\section{„,Nachsichtige Unzufriedenheit“ mit mangelnder Hochschuldidaktik}

Alle Studierenden sind, unabhängig von ihrer sozialen Herkunft, dadurch unterprivilegiert, dass sie tendenziell in einem hochschuldidaktischen Vakuum lernen. Ihm liegt die im I. Kapitel dargestellte anti-pädagogisch-didaktische Tradition zugrunde, die mit der Ordinarienuniversität und mit dem Primat der Vorlesung verbunden ist (I. 1.). Aus der in dieser Tradition verankerten Einstellung heraus stellte der Orientalist C. H. Becker - in den 1920er Jahren preußischer Kultusminister - spöttisch-herablassend die Frage: „Hochschulpädagogik - welchen deutschen Professor überläuft es nicht kalt bei diesem Wort? ${ }^{* 30}$ Wie kontinuierlich diese Auffassung, die Becker und mit ihm die Professorenschaft der Weimarer Republik aus dem Kaiserreich übernahm, auch in der Bundesrepublik weitergeführt wurde, zeigte sich besonders deutlich seit Beginn der 1970er Jahre. Damals wurden die hochschuldidaktischen Impulse entschieden zurückgedrängt, die seit Ende der 1960er Jahre von der Studentenbewegung ausgegangen waren. ${ }^{31}$ Auf welch unfruchtbaren Boden die Saat der punktuell geschaffenen hochschuldidaktischen Zentren zeigte sich schon sehr früh daran, dass selbst in ,neuerungsverdächtigen Fächern“ wie der Soziologie nur vereinzelt Versuche unternommen wurden, aus dem Trott der frontalen Lernformate herauszukommen. ${ }^{32}$

30 Ders., Gedanken zur Hochschulreform, 1919; zit. nach: Peter Glotz, Stilwandel in der Studienreform, in: Bildung und Politik, 1980/Nr.1; in: G. Braungart u. a. 1981: 42.

31 Im Zentrum standen Initiativen der sogenannten Bundesassistentenkonferenz. Ihre Reformvorstellungen führten zur Einrichtung hochschuldidaktischer Zentren. (Vgl. zur Bestandsaufnahme: Senatskommission der DFG für Hochschuldidaktik, Die Lage der Hochschuldidaktik: Bericht und Materialien, Hamburg 1980/Hochschuldidaktische Materialien 74; vgl. auch Kap.I. 2.)

32 Vgl. etwa die Ergebnisse einer Erhebung aus dem Jahr 1973, nach der nur eine kleine Minderheit der befragten Soziologen (alle Professoren der soziologischen Diplomstudiengänge plus zwei Vertreter des Mittelbaus pro Professor) mit neuen Lehrformaten wie Blockseminaren, Veranstaltungen mit Praktikern, Praktika u. a. experimentierte. 
Für die 1980er Jahre ist auffällig, dass der Rotstift der ter mit Vorliebe hochschuldidaktische Einrichtungen auslöschte. Zum Beispiel wurde das hochschuldidaktische Zentrum der Hochschule der Bundeswehr in München mit der Begründung aufgelöst, man könne sich dem Zwang von Stelleneinsparungen nicht entziehen. ${ }^{33}$ Als unverkennbares Zeichen für die fehlende Durchsetzungskraft der Hochschuldidaktik darf auch gewertet werden, dass der Arbeitskreis Hochschuldidaktik (AHD), der im Gefolge der Studentenbewegung 1971 entstanden war, immer eine Schattenexistenz führte. Dasselbe gilt für die Deutsche Gesellschaft für Hochschuldidaktik (dghd), die 2008 an die Stelle der AHD trat. Sie und ihre Mitglieder konnten ihre durchaus beachtlichen Forschungsergebnisse in den aktuellen Diskussionen der Hochschulreform nicht zur Geltung bringen. ${ }^{34}$

Wie schon in der Einführung (4.) betont wurde, steht hinter der traditionellen Weigerung, sich über pädagogisch-didaktische Probleme des Lernens Gedanken zu machen, die fehlende Vertrautheit mit neuronal vermittelten Lernprozessen. In der Sicht einer neurobiologisch uninformierten Kybernetik ist es die Annahme, dass von Sendern ausgehende Informationen eins zu eins von Empfängern aufgenommen werden können. ${ }^{35}$

Was die Studierenden betrifft, so

auch sie sich nicht auf dem Stand einer neurobiologischen Forschung, für die klar ist, dass an Hochschulen nach Prinzipien instruiert wird, die den neurobiologischen Einsichten zum schöpferisch-konstruktiven Gehirn widersprechen. Bei der studentischen Beantwortung von Fragen zur Didaktik darf daher nicht unterstellt werden, dass die Befragten präzise didaktische Kriterien anlegen. Ihr Antworthorizont wird durch einfache Erwägungen bestimmt, die hinter dem noch zu illustrierenden Vorlesungs-Lob einerseits, hinter der Vorlesungs-Kritik andererseits erkennbar sind (vgl. Kap. IV.2.). Nun ist indessen der didaktische Notstand in einem System weitgehend unausgebildeten Lehrpersonals so groß, dass dieser auch inkompetenten Studierenden nicht verborgen bleiben kann.

Vgl. Joachim Hamers, Die Universitätsausbildung von Sozialwissenschaftlern, Frankfurt/New York 1978: 135.

33 Vgl. Peter Zimmermann, Nachruf auf das Hochschuldidaktische Zentrum der Hochschule der Bundeswehr München, in: Hochschulausbildung, 1983/H.1: 3-17; hier: 12.

34 Dies gilt vor allem für die noch in Zeiten des AHD entwickelte Umstellung vom inhaltlich orientierten Lehren auf das Lernen des Lernens (vgl. besonders Behrendt 2005).

35 Inzwischen gibt es findige Journalisten, die sich in der einschlägigen Fachliteratur kundig machen, um die Mechanismen des Informationszeitalters zu durchschauen. Sie begreifen und versuchen die Einsicht weiterzugeben, dass speziell das Verhältnis zwischen Lehrpersonen und Lernenden/Studierenden nicht nach dem Sender-Empfänger-Prinzip verstanden und die Kommunikation nicht als „Maschinisierung“ missverstanden werden darf (vgl. etwa: Schirrmacher 2009: hier bes.: 214f.). 
Das zeigte sich in zunächst allgemein gehaltenen repräsentativen Umfragen zur Studiensituation in der zweiten Hälfte der 1980er Jahre daran, dass diese auf einer Skala von 1 (=sehr gut) bis 6 (=sehr schlecht) - bei einem Mittelwert zwischen 3,6 und 4,5 - in der Tendenz für ,,schlecht" gehalten wurde. ${ }^{36}$ Als die vom Bundesministerium für Bildung und Forschung beauftragte Konstanzer Forschungsgruppe um Tino Bargel ihre Fragen differenzierter auf die didaktische Qualität der Studiensituation bezog, ergab sich, dass über viele Jahre hinweg eine beträchtliche studentische Minderheit von ungefähr 20\% ihre Unzufriedenheit zum Ausdruck brachte. Das gilt auch noch für die nach der Bologna-Reform befragten Bachelor-Studierenden (Befragung im WS 2012/13)..$^{37}$

Woran liegt es nun aber, dass komplementär dazu immer deutlich mehr als die Hälfte der Befragten gute Bewertungen abgaben. Bei der zuletzt erwähnten Befragung waren es $60 \%$. Der Grund kann nur darin liegen, dass hier der symbolisch-diskursive Kontext durchschlägt. Aus seinem Repertoire der Winzigkeit kommen offenbar Botschaften, die geeignet sind, die Studierenden dazu anzuhalten, gegenüber ihren didaktisch wenig überzeugenden Lehrpersonen nachsichtig zu sein. Nur so erklärt sich, dass sie ihre Unzufriedenheit hinter einem positiven Votum verstecken.

\section{„Nachsichtige Unzufriedenheit“ mit mangelnder Betreuung und Beratung}

Was die studentischen Einschätzungen von Betreuung und Beratung angeht, so muss von Daten ausgegangen werden, die sich auf den Umfang der Kontakte beziehen, die zwischen den Studierenden und ihren Lehrpersonen bestehen. Aus den vorliegenden Erhebungen geht hervor, dass die gegen Ende der 1980er Jahre ermittelten Daten tendenziell sowohl mit den Daten der zum Teil nur beschränkt repräsentativen Befragungen der 1960er, 1970er und 1980er Jahre $^{38}$ als auch mit dem Ergebnis der Befragungen von Bachelor-Studierenden $^{39}$ übereinstimmen. Durchgehend haben zwischen $65 \%$ und $70 \%$ der Studierenden „selten oder nie“ Kontakte.

Entgegen der Erwartung, dass dies eher zu unzufriedenen Studierenden führen müsste, wird die Betreuungs- und Beratungslage von 50\% der Befragten -

36 Bargel u. a. 1989: Tabelle 3: 19 (Bewertung der Qualität der Lehre aus der Sicht der Studierenden).

37 Ramm u. a. 2014: Abb. 19: 49.

38 Bargel u. a. 1989: Tabelle 4: 21 (Kontakte von Studierenden zu Hochschullehrern Professoren und Assistenten - im Zeitvergleich an Universitäten).

39 Bargel u. a. 2009: 43 (,Stagnation auf niedrigem Niveau“); Ramm u. a. 2014: Abb. 19: 49. 
mit Abweichungen von ungefähr 10\% nach oben und unten - positiv eingeschätzt. Bei den Bachelor-Studierenden des WS 2012/13 sind es $60 \%{ }^{40} \mathrm{Da}$ nicht angenommen werden kann, dass dieses Urteil auf die Arbeit spezieller Beratungseinrichtungen zurückgeht, halten sich die Studierenden für besser betreut und beraten, als sie es tatsächlich sind. Parallel zur überwiegend positiven Beurteilung der didaktischen Qualität des Lehrpersonals liegt das offenbar auch im Themenbereich der Betreuung und Beratung daran, dass die auf Unterordnung gerichteten Botschaften des symbolisch-diskursiven Kontexts wirksam sind. Den ungefähr 20\% der Befragten, die ihren Unmut äußern, stehen immer mehr als doppelt so viele Studierende gegenüber, die sich dazu gedrängt fühlen, ihrer Unzufriedenheit Zügel der Nachsicht anzulegen. ${ }^{41}$

\section{„Nachsichtige Zufriedenheit" in punktueller Nahkommunikation: Privilegien einer kleinen studentischen Minderheit}

Es gehört zu den Opportunitätsstrukturen der Massenuniversität und ihrer Betreuungsrelationen, dass Professorinnen und Professoren nur sehr wenigen Studierenden nahekommen können. Das galt beispielsweise für eine Studentin der Politik-, Geschichts- und Rechtswissenschaft. Sie war im 7. Semester und erzählte im Interview nicht ohne Stolz, dass sie - in ihrer eigenen Formulierung - eine der ,behaglichen Nischen für erfolgreiche leistungsorientierte Studenten“ gefunden habe. Sie berichtete von einer „Tätigkeit an einem sehr guten Lehrstuhl und einer sehr persönlichen und vertrauensvollen Zusammenarbeit mit Professor X“. In diesem Fall beruhte die Nähe zum einzelnen Pro-

40 Bargel 1989: 22; Bargel 2009: Tab. 53/54: 69; Ramm u. a. 2014: Abb. 19: 49.

41 Sofern präzise und differenzierte Fragen gestellt werden, kann sich die studentische Nachsicht als begrenzt erweisen. Dies wird beispielsweise durch die Ergebnisse einer Untersuchung am Fachbereich Politische Wissenschaft (Otto-Suhr-Institut) belegt, in der 163 Studierende der Zwischenprüfungs- und Examensjahrgänge 1989/90 die Leistungen von 100 Professorinnen und Professoren sowie Mitarbeiterinnen und Mitarbeitern zu bewerten hatten. Das Ergebnis ist zweifellos nicht repräsentativ, in der Grundtendenz aber doch aussagekräftig. Die akademische Ausbildungskommission des Otto-Suhr-Instituts, die die Fragen stellte, ging von einem breiten Betreuungsfeld aus. Zu diesem gehörten die Einführung in wissenschaftliches Arbeiten, die Betreuung von studentischen Arbeitsgruppen, die Besprechung von Hausarbeiten sowie die Vorbereitung auf Zwischenprüfung und Examen. Dabei ergab sich zusammenfassend folgender Befund: „Über 60 Prozent der Befragten fühlten sich überhaupt nicht oder nicht genügend in das wissenschaftliche Arbeiten eingeführt, nur 20 Prozent der kursbegleitenden Arbeitsgruppen wurden betreut, nur ca. 30 Prozent aller Hausarbeiten wurden besprochen und das Gespräch darüber als einigermaßen positiv bewertet, nicht einmal die Hälfte der Diplomanden sieht sich im Examen ,gut' oder ,mittelmäßig ' betreut.“ (Peter Grottian, in: Grühn u. a. 1992: 28.) 
fessor auf dem kulturellen Kapital (Bourdieu), das die Studentin aus ihrem bildungsnahen Elternhaus mitbrachte. Möglichkeiten der Annäherung bieten sich punktuell aber auch einzelnen Studierenden aus bildungsfernen Schichten. Es lässt sich beobachten, dass für Professorinnen und Professoren beispielsweise Studierende ,interessant“ sind, die auf den Hintergrund einer kinderreichen Familie studieren oder darauf verweisen können, dass sie nach einem schwierigen, aber bravourös absolvierten Bildungsweg eine gewerkschaftliche Karriere anstreben, sich auf anstrengende Aufgaben in der alternativen Jugendarbeit oder im kirchlichen Entwicklungsdienst vorbereiten.

Solche Studierenden, die sich aus der großen Masse herausheben, bilden eine kleine Minderheit. Zugang wer zum persönlichen und politischen je einzelner Lehrpersonen passt und ihren Leistungsanforderungen entspricht. Erste zarte Bande entstehen in Sprechstunden, die länger als üblich dauern, bei der Besprechung von Hausarbeiten und Übungsklausuren, zu der sich diejenigen, die das Lernen anleiten, mehr Zeit als sonst nehmen. Schon viel haben diejenigen erreicht, die sich einen Platz im Gesichts- sowie Namensgedächtnis erobert haben. Da kann es dann passieren, dass plötzlich eine Hilfskraft- oder Tutor(innen)stelle frei und mit dem bekannten Gesicht assoziiert wird. Vornehmlich in naturwissenschaftlich ausgerichteten Fächern haben einzelne Studierende das Glück, in Forschungsprojekte einbezogen zu werden, in denen sie in persönlicher Nähe zu ihren professoralen Betreuungspersonen lernen.

Der in allen Details offenliegende Fall des Plagiators Karl-Theodor Freiherr von und zu Guttenberg, auf den im nächsten Abschnitt noch einzugehen ist, macht nicht nur den privilegierten Erwerb von Doktortiteln öffentlich. ${ }^{42}$ Schon auf früheren beginnend mit dem Erwerb von Seminarscheinen und beim erfolgreichen Abschluss von (Zwischen-)Prüfungen wird erkennbar, wie in „Séparées“ des Hochschulsystems privilegierte Lernkarrieren ablaufen können. Illustrative Details des Falls Guttenberg zeigen dies unmissverständlich:

Ein bekannter Jura-Professor (Häberle) trifft auf einen strahlend-selbstbewussten Kandidaten, den er vermutlich schon von früher kennt. Er passt ins Klima eines zugleich seriösen, stilvollen und heiteren Lernbetriebs. Obwohl kein notwendiger Bestandteil des Einstiegskontakts, ist es vermutlich hilfreich, dass Guttenberg aus adeligem Hause stammt und auch schon seine politischen Talente erkennen lässt. Entscheidend ist jedoch, dass dieser Kandidat im

42 Die folgenden Beobachtungen stützen sich auf Berichte und Kommentierungen, die von Februar bis Juni 2011 unterschiedlichen Medien zu entnehmen waren. (Vgl. als besonders aufschlussreich den Bericht über ein Interview, das Heribert Prantl mit dem Doktorvater Guttenbergs, Peter Häberle, führte; in: Süddeutsche Zeitung, 9./10.4.2011). 
engsten Professoren-Umfeld durch seine fachbezogene mündliche Überzeugungskraft in Übungen und Seminaren, durch seine allgemeine sympathische Ausstrahlung und dadurch auffällt, dass er den im Mitarbeiter- und speziell im Doktorandenkreis vorherrschenden Ton trifft. Punktuell darf und muss der Kandidat durch originelle Abweichungen auffallen. Ihre Einbettung in den im Ganzen fraglos geltenden Zustimmungsdiskurs bedarf jedoch sorgfältiger Kalkulation. Der Kandidat muss nach einiger Präsenzzeit gelernt haben, sich fast traumwandlerisch sicher zu bewegen. Ist auf diese Weise ein unbezweifelbares $\mathrm{V}$ entstanden, so ist der Punkt erreicht, an dem der Kandidat so gut wie sicher sein kann, dass er seine Ziele, speziell sein Promotionsziel, erreichen wird. Schriftliches Leistungsvermögen wird vom vielfach eingeübten allgemeinen und speziell vom mündlichen $\mathrm{V}$ abgeleitet. Kommt es bei Diplom-, Staats- und Doktorprüfungen zur Bewertung von mündlichen Leistungen, so sind auch sie tendenziell schon vor der Prüfung klar. Diese Erklärung dafür, dass Plagiate möglich sind, bedarf im folgenden Abschnitt noch der Präzisierung und Erweiterung.

Studierende, die in Nahkommunikation mit den Lehrpersonen als Minderheit Privilegien genießen, gab es auch schon in früheren Zeiten. Ihnen stand jedoch keine übergroße Mehrheit gegenüber, wie sie die Massenuniversität kennt. Es charakterisiert die in sich abgestufte kleine Minderheit, dass sie sich ihrer Vorzugsstellung bewusst ist und diese zu würdigen weiß. Die der allgemeinen Lern-Konstellation, die auch diese Studierenden zu spüren bekommen, beeinträchtigen ihre allgemeine Zufriedenheit kaum. Diese ist so dominierend, dass Missstände mit ihrer wohlwollenden Nachsicht rechnen können.

\section{Privilegierte Promovierende und ihre Plagiatspraxis: „Altruistic Punishment" in gestörten Vertrauensbeziehungen}

Zur kleinen Minderheit privilegierter Studierender gehören diejenigen, die in der Massenuniversität Betreuerinnen oder Betreuer die ihnen helfen, durch den Erwerb eines Doktortitels eine (denkbare) akademische Laufbahn zu fundieren oder eine außeruniversitäre Karriere zu absolvieren. Eine skizzenhafte Untersuchung der Plagiatspraxis macht freilich deutlich, dass die Bereitschaft der Promovierenden, sich der schlechten Betreuungslage zu unterwerfen, ihre Grenzen hat.

Kaum Platz haben Plagiate in den erwähnten naturwissenschaftlich geprägten Teamarbeitsfächern. Hier sind sich Lehrpersonen und Lernende nahe, weil sie beispielsweise als Chemikerinnen und Chemiker im Labor, als Physikerinnen und Physiker in Experimenten oder als Neurowissenschaftlerinnen und -wissenschaftler bei der Erhebung und Verarbeitung von Scan-Daten gemeinsam Projekte durchführen. Der Erfolg solcher Projekte ist von den Leistun- 
gen aller Beteiligten abhängig. Speziell die Arbeitsbeziehung zum prüfenden Personal garantiert, dass dieses auf die Beiträge eigener Doktorandinnen und Doktoranden ein besonders wachsames Auge hat. Dies ist von Seiten der Naturwissenschaft immer wieder betont worden.

Demgegenüber handelt sich in den Fällen, die in der Bundesrepublik der Jahre 2011/12 Aufsehen erregten, um Plagiate Einzelner, die in großer Distanz im Verhältnis zwischen Betreuungspersonen und Betreuten entstanden. Es ist diese Distanz, die Voraussetzungen dafür schafft, dass sich die Betreuten vor allem über die folgenden zwei im Wissenschaftsbetrieb etablierten Prinzipien hinwegsetzen: Erstens wird das Gebot verletzt, dass wörtliche Übernahmen aus Büchern, Aufsätzen und Quellen als Zitate zu kennzeichnen und zu belegen sind. Zweitens wird das Gebot missachtet, dass Textpassagen, die in verkürzter oder zusammenfassender Version präsentiert werden, als „Anleihen“ auszuweisen sind (Weber 2011: 31-35).

derbundesrepublikanischen Skandalchronik, der hier nicht im Einzelnen nachgegangen werden kann, waren: Karl-Theodor von und zu Guttenberg (Verteidigungsminister), Silvana Koch-Mehrin (Europa-Abgeordnete der FDP), Annette Schavan (Wissenschaftsministerin) und Margarita Mathiopoulos (FDP-Beraterin), deren Plagiat auf das Jahr 1986 zurückgeht. Spektakulär angeführt von Guttenberg, verletzten alle die genannten Prinzipien. In der Hauptsache wurde ihnen vorgeworfen, das Vertrauensverhältnis zu ihren Betreuungspersonen planmäßig verletzt und auf diese Weise kalkuliert betrogen zu haben.

Besonders vehement wies Guttenberg diesen Vorwurf zurück. ${ }^{43}$ In der guten Gesellschaft anderer Plagiatorinnen und Plagiatoren machte er geltend, dass er - neben Engagement - sehr viel Arbeitskraft, Kreativität und Zeit in sein Dissertationsprojekt gesteckt habe. In seinen Augen war die Vergabe des Doktortitels durch diesen Aufwand gerechtfertigt.

In der deutschen Diskussion wird die unzulängliche Betreuungslage der Promovierenden eher heruntergespielt. Sie besteht zum einen und völlig unbestreitbar darin, dass deutsche Professorinnen und Professoren auf eigene Forschungs- und Publikationsvorhaben konzentriert sind. Zum anderen und damit zusammenhängend beruht die Vernachlässigung der Promovierenden darauf, dass ihre Prüferinnen und Prüfer wenig Zeit für die Lektüre der ihnen vorgelegten Texte aufwenden. Das gilt nicht für Lehrpersonen in den sogenannten Orchideenfächern, die nur mit wenigen Studierenden zu tun haben, deren Betreuung daher zum Lebensinhalt machen können und wollen. Demgegenüber stehen alle hier exemplarisch aufgeführten Fälle dafür, dass die Missachtung 
der genannten Prinzipien - auch ohne Einsatz der neuesten computerntechnischen Hilfsmittel - bei genauer Lektüre hätten auffallen müssen. Speziell im Fall Guttenberg ist klar, dass der Doktorvater Häberle sich als vielbeschäftigter Gelehrter und international bekannter Verfassungsjurist keine Zeit dazu nahm, Guttenbergs Text detailliert durchzugehen.

In angelsächsischen Analysen wird die schlechte Betreuungslage als wichtige Grundlage der Plagiatspraxis stark betont. In einer zusammenfassenden und zugespitzten Präsentation vielfacher Mängel, unter denen die Promovierenden leiden, leuchtet die These ein, dass ,Studenten schlechte Pädagogik mit (mehr) Plagiarismus beantworten“ (Althaus 2011: 113). Es ist diese These, die sich mit neurobiologischen Einsichten in die Beziehungen von Interaktionspartnern in Verbindung bringen lässt.

In neueren Studien geht es darum, überkommene sozialphilosophische Positionen mit den vorliegenden neurowissenschaftlichen Erkenntnissen abzugleichen. Diese zwingen zu der Einsicht, dass Gehirne als „Beziehungsorgane“ betrachtet werden müssen (Fuchs 2007). Sie ermöglichen soziale Kontakte unterschiedlicher Intensität. Dabei geht es um teim Zusammenhang von Familien, Stämmen und vielen gesellschaftlichen Institutionen, unter ihnen auch das hier interessierende Hochschulsystem.

In Prozessen tierisch-menschlicher Evolution entwickelten sich die Sozialbeziehungen in einer kooperativen Alltagspraxis, die bis heute das Überleben absichert und im Genom verankert wurde (Pinker 2003; Wuketits 1993). Auf diesem baut die Architektur der Gehirne auf, die sich als Beziehungsorgane durch eine besonders breite emotionale Grundlage auszeichnen. Die Einbettung der Sozialbeziehungen in das sogenannte limbische System und sein Umfeld hat der Neurobiologe Antonio Damasio in einer neueren Publikation ausführlich analysiert und zugleich den ,switch“ beschrieben, über den aus unbewussten ,emotions“ bewusst registrierte und benutzte Antriebskräfte werden (Damasio 2011: bes. 129ff.; früher schon - im Anschluss an Spinoza Damasio 2005: bes. 205).

Zum limbischen System gehören neuronale Netzwerke, in denen die Neigung abgestützt ist, Interaktionspartnerinnen und -partnern Vertrauen zu schenken und mit ihrem Vertrauen zu rechnen. (Zusammenfassend und mit Hinweis auf neurobiologische Trägerstrukturen: Fehr 2009: 215ff.) Dabei geht es vor allem darum, im gegenseitigen Umgang auf Gleichbehandlung zu achten und fair zu sein. Wichtig sind neuronale Schaltstellen, die einerseits für die empathische Fähigkeit zuständig sind, sich in das Denken und Fühlen von Mitmenschen hineinzuversetzen. Beteiligt sind immer auch Areale, in denen Belohnungserwartungen erzeugt und ihre Einlösung oder Enttäuschung registriert werden.

Diese vertrauensorientierten Sozialbeziehungen werden unter anderem 
im Rahmen der sogenannten „Neuroeconomics“ untersucht (Fehr, ebda.). In sorgfältig ausgewählten Versuchsanordnungen spielerischer Kommunikation wird die Abwicklung von Geld(leih)-Geschäften erforscht. Dabei zeigt sich, dass die Interaktionspartnerinnen und -partner nicht ausschließlich von ökonomischen Eigeninteressen geleitet werden. Im Spiel ist immer ein soziales Interesse an den Mitspielerinnen und Mitspielern und daran, dass gegenseitige Vertrauensbeziehungen sowohl erzeugt als auch aufrechterhalten werden. Wer Angebote macht, hat im Auge, bis zu welchem Grade sie für die andere Seite als fair akzeptiert werden können. Umgekehrt werden auch faire Antworten erwartet. Wo sie fehlen, gibt es umfangreiche Belege für die Neigung, Unfairness des Gegenübers auch dann zu bestrafen, wenn dies den eigenen ökonomischen Interessen widerspricht. Dabei handelt es sich um Reaktionen, die als „Altruistic Punishment" bezeichnet werden: Gemeint ist, dass Spielende, die gegen das Prinzip der Fairness verstoßen, damit rechnen müssen, dass sich ihre Mitspielenden dafür revanchieren. Dabei lässt sich nur schwer klären, bis zu welchem Grade dieses Revanche-Prinzip durch Areale des präfrontalen Cortex bewusst eingelöst wird oder aber einem unkontrollierten Mechanismus folgt. Versucht man diese Einsichten auf das Verhältnis zwischen Promovierenden und ihrem Betreuungspersonal zu übertragen, so lässt sich die Plagiatspraxis folgendermaßen interpretieren:

Plagiate stellen von Seiten der Betreuten zweifellos einen Bruch des Vertrauensverhältnisses zu ihren Betreuungspersonen dar. Gegen die geltenden Regeln legen Erstere ohne hinreichende Belege Text-Stücke vor, die nicht von ihnen selbst stammen. Umgekehrt tragen aber auch Zweitere zum Vertrauensbruch bei. Sie werden zum Objekt von „Altruistic Punishment“, weil sie, auf ihre eigenen Interessen konzentriert, die Ansprüche der Betreuten auf Fairness enttäuschen. In großer Distanz zu den Promovierenden fehlt es im Allgemeinen an hinreichender Aufmerksamkeit, im Speziellen an sorgfältiger Text-Lektüre, von der eine leistungsgerechte Bewertung abhängig ist. Insoweit steckt in Plagiaten ein Moment nachvollziehbaren „Altruistischen Protests“ gegen fehlende Reziprozität. Betrugsvorwürfe gegenüber den Betreuten werden damit zwar nicht beseitigt, jedoch dadurch relativiert, dass das betreuende Personal an der Störung des Vertrauensverhältnisses beider interagierender Personen Anteil hat. Das gilt insbesondere für den Fall, dass Bequemlichkeit oder Schlamperei zu kleineren Verstößen gegen die Zitier- und Beleg-Regeln führen. Bei darauf bezogenen Entscheidungen darüber, ob der Entzug von Doktortiteln als gerechtfertigt angesehen werden muss, kommt es darauf an, ob und bis zu welchem Grade das betreuende Personal seine Fürsor gegenüber den Promovierenden erfüllt hat.

Stimmen diese neurobiologisch informierten Beobachtungen und Überlegungen, so passen die Plagiate, die auf der Endstufe des Lernens an Hochschulen 
praktiziert werden, zur allgemeinen Lern-Konstellation. Sie ist durch eine deBeratungs- und Betreuungslage gekennzeichnet, die auf Seiten der Promovierenden zu einer Plagiatspraxis führen kann, in der die Bereitschaft, sich ein- und unterzuordnen, in ,altruistisches Protestverhalten“ umkippt.

\section{Zusammenfassung}

Der Sozialpsychologe Horst Rumpf, der aus der Studentenbewegung kam und sich Ende der 1970er Jahre um alternative Lernformate bemühte, beklagte sich darüber, dass es an der Universität zu einer ,szenisch vermittelten Lähmung von Subjekten" komme (Rumpf 1978: 31-79). Das war eine zugespitzte und in organologisch-physiologischer Ausdrucksweise verkürzte Charakterisierung der allgemeinen Lern-Konstellation, wie sie hier beschrieben wurde. Rumpf trifft aber doch ihren Kern: Studierende werden in ihren Lernaktivitäten gelähmt, als ob sie in ihrer körperlichen Beweglichkeit, beispielsweise durch einen Schlangenbiss, gestoppt würden. Diese Konstellation kann in neurobiologischer Perspektive folgendermaßen zusammengefasst werden:

Die aus dem Macht-Diskurs kommenden und neuronal vermittelten Eindrängen die große studentische Mehrheit in die Winzigkeit, die sie von denen weit entfernt, die das Lernen anleiten. Die Lernenden sich in einem Arrangement der Unterordnung, zu dem auch die erwähnten Neuromodulatoren beitragen. Die bei den Lernenden erzeugte lähmende Wirkung beruht darauf, dass es in den Lernbeziehungen an Nahkommunikation und damit an günstigen Voraussetzungen für ertragreiches Lernen fehlt. 


\section{Spezielle Lern-Konstellation im Vorlesungsbetrieb}

Vorgelesen wird nicht nur in Hörsälen. Frontale Reden werden auch in Seminar- und Übungsräumen gehalten. Im Besonderen gilt, dass auch studentische Tutorinnen und Tutoren gegenüber Mit-Studierenden einen Vorlesungs-Gestus praktizieren, den sie ganz selbstverständlich übernehmen. Wenn im Folgenden Vorlesungen im engeren Sinne als Hörsaal-Vorlesungen betrachtet werden, so gelten die angestellten Beobachtungen und Überlegungen prinzipiell auch für andere Anwendungen des Vorlesungsprinzips, ohne dass diese hier in ihren besonderen Ausformungen untersucht werden können (vgl. EXKURS 2).

Das Vorlesungsprinzip, das hier in den Rahmen des deutschen Obrigkeitsstaats eingeordnet wird, stieß in der zweiten Hälfte der 1960er Jahre kurzfristig auf studentischen Widerstand. Es kam zu Korrekturen antiquierter Umgangsformen im Verhältnis der Studierenden zu den universitären Amtsträgern einerseits, in den Beziehungen zwischen den Lernenden untereinander andererseits. Spätestens seit dem Hochschulrahmengesetz des Jahres 1974 und seiner gesetzgeberischen Umsetzung in den Bundesländern war jedoch die Welt der Ordinarienuniversität wieder in Ordnung. Informell änderte sich am sogenannten Lehrstuhlprinzip nichts. In der Massenuniversität, die differenzierte Studiengänge anbietet, bedarf es zwar vielfältiger Abstimmungen unter den „Lehrstuhlinhabern“. Bei ihnen konzentriert sich jedoch die fächer-

Bestimmung von Lerninhalten, auch wenn diese in stärker verschultem Design angeboten werden. Von ihnen werden Prüfungen abgehalten und administriert, die zu unterschiedlichen Studienabschlüssen - bis hin zur Promotion - führen. In ihren Händen liegt die universitäre Personalpolitik, die darüber entscheidet, wer die Ämter übernimmt, die im Bereich der technischen Dienstleistungen und auf der Ebene abgestufter „Lehrfunktionen“ zu vergeben sind.

Indessen haben die Ordinarien gegenwärtiger Hochschulen ihre gesellschaftliche Leitfunktion zweifellos verloren. Die Hochschullehrerinnen und -lehrer unterliegen den Zwängen eines komplexen Zusammenspiels vielfältiger ökonomischer und politischer Faktoren. Geblieben ist freilich das fast ungetrübte Selbstverständnis ,,vorlesender Mandarine“, die es für ihr Privileg und auch für ihre halten, sich vor studentischem Publikum wöchentlich in spektakulärer Weise zu artikulieren. Eher diffus bleibende Zweifel am Lernertrag von Vorlesungen werden meist stillschweigend durch die Überzeugung zerstreut, dass es ohne das überlieferte Vermittlungs-Instrument nicht möglich wäre, die massiv gewachsene Nachfrage im Massenbetrieb der Hochschulen zu befriedigen. Dabei fehlt es an didaktischer und speziell am Nachdenken darüber, dass sich mit dem Vorlesungsprinzip un- 
terschiedliche Ansprüche und Erwartungen sowohl auch formaler Art verbinden.

Im Ganzen ist es - fächerübergreifend - dabei geblieben: „Worüber lesen Sie in diesem Semester?", fragt der hochschulferne Nachbar die Professorin oder den Professor von nebenan. „Welche Vorlesungen habt ihr belegt?“, wollen die Menschen im Umfeld von Studierenden wissen.

\section{Vorlesungs-Idealtypen}

Um das Vorlesungs-Lob und die ihm gegenübergestellte Vorlesungs-Kritik sowie die Vorlesungspraxis und die an Vorlesungen gerichteten Erwartungen einordnen, präzisieren und in neurobiologischer Sicht bewerten zu können, werden die hervorstechenden Merkmale der akademischen Rede zu zwei übergeordneten

Idealtypen und drei diesen untergeordneten formalen Idealtypen zusammengefasst. Sie sind alle - im Sinne von Max Weber - beschreibend und nicht normativ gemeint. Diese Typologisierung dient dazu, Vorlesungen als „Mischprodukt“ unterschiedlicher Merkmale zu erkennen.

Der erste inhaltl

Vorlesungs-Typ lässt sich als Idealtyp allgemeiner oder spezialistischer Instruktion bezeichnen (Instruktions-Vorlesung). Einerseits werden Lernende über Inhalte bestimmter Fachgebiete überblicksartig informiert. Fachleute tragen - oft unter der Etikette Einführung - in zusammenfassender Weise vor, was sie in ihren Gebieten für besonders wichtig und wissenswert halten. Das gilt beispielsweise für eine Vorlesung des Titels „Einführung in die Neurobiologie“. Hier wird allgemein über die historische Entwicklung dieses Forschungszugriffs, über die eingesetzten Forschungsmethoden und über wichtige Forschungsergebnisse berichtet. Andererseits bezieht sich die Instruktion innerhalb bestimmter Fachgebiete auf ausgewählte Spezialthemen. Beispielsweise wird im Bereich der Neurobiologie die Gedächtnisforschung behandelt: Erläutert werden sowohl die neuronalen Grundlagen unterschiedlicher Gedächtnissysteme als auch ihr kompliziertes Zusammenspiel, in dem von außen kommende Impulse auf unbewusste und teils bewusste Weise verarbeitet werden. Diesem Vorlesungs-Typ liegt ein reduziertes, auf bloße Stoffvermittlung gerichtetes Verständnis von Lernen zugrunde.

Demgegenüber baut der zweite

Idealtyp auf einem breiteren Verständnis von Bildung und Lernen auf. Gemeint sind frei vorgetragene Reden, die im Übergang vom 18. zum 19. Jahrhundert von akademischen Lehrern wie Fichte, Schleiermacher und Hegel praktiziert wurden. Dabei ging es darum, sich vom bloßen Ablesen eines Manuskripts (lectio) abzusetzen (Apel 1998: 60; Apel 1999: 22-26). Dieses Vorlesungs-Format zielt nicht in 
erster Linie auf die Vermittlung von Wissensstoff. Vielmehr geht es darum, Aufmerksamkeit zu mobilisieren und zu schärfen. Lernende sollen irritiert und durch Denkanstöße dazu gebracht werden, sich sowohl auf fachübergreifende als auch auf

Probleme einzulassen. Es wird vorgeführt, was es heißt, im Bezugsrahmen der Philosophie, der Mathematik, der Politikwissenschaft ... zu denken (Huber 2011: 121). In diesem Sinne lässt sich der zweite inhaltliche Idealtyp als Impuls-Typ bezeichnen (Impuls-Vorlesung).

Der erste formale Vorlesungs-Typ lässt sich als Idealtyp charismatischer Faszination bezeichnen. Das Charisma hat zwei zusammengehörige Bestandteile. Einerseits faszinieren besondere rhetorische Fähigkeiten, eine bestimmte Tonlage der Stimme und eine einfache, klare sowie schlüssige Artikulationsweise. Andererseits besteht das Charisma aus besonderen Merkmalen persönlicher Ausstrahlung, die auch bei mangelnder rhetorischer Begabung wirksam sein können: Vorlesende vermitteln den Eindruck, in hohem Grade lebendig, seriös, glaubwürdig oder vertrauenswürdig zu sein. Worauf solche Momente der Ausstrahlung zurückgehen, ist oft schwer zu sagen. Es mag an einer geraden oder krummen - Körperhaltung, auch an den Bewegungen einzelner Körperteile (Kopf, Augen, Hände, Schultern) oder am Muskel- und Mienenspiel des Gesichts liegen. Eine beispielhafte Beschreibung schwer barer charismatischer Eigenschaften liefert der Dichter Heinrich von Kleist, der 1805 in Königsberg den Finanzwissenschaftler Christian Jakob Kraus vorlesen hörte. Er sei „ein kleiner unansehnlich gebildeter Mann, der mit fest geschlossenen Augen, unter Gebärden, als ob er im Kreisen begriffen wäre, auf dem Katheder sitzt; aber wirklich Ideen mit Hand und Fuß, wie man sagt, zur Welt bringt. Er streut Gedanken, wie ein Reicher Geld aus, mit vollen Händen, und führt keine Bücher bei sich, die sonst gewöhnlich, ein Nothpfennig, den öffentlichen Lehrern zur Seite liegen.“ (Zit. nach: Bisky 2011)

Der zweite formale Idealtyp beruht auf einer verbreiteten menschlichen Neigung, sich vor anderen Menschen und in der Abgrenzung von ihnen hervorzutun. Er lässt sich als Vorlesungs-Typus snobistischer Fachidiotie umschreiben. In wichtigtuerischer Pose wird Wissen in exotisches Vokabular verpackt, das den Lernenden nicht vertraut ist und sie mürrisch und zumindest unsicher macht. Eine unnachahmlich gelungene Illustration hiervon bietet der Filmemacher Alexander Kluge, der in seinem Film „Abschied von gestern“ (1966) seine verschiedensten der bundesrepublikanischen Gesellschaft aussetzt. Unter anderem bringt Kluge Anita G. mit der deutschen Nachkriegs-Universität in Berührung. Seismographisch wird ihre Belehrung durch einen kauzigen Professor aufgezeichnet. Wirres Jonglieren mit berühmten Namen und sozialwissenschaftlicher Fachterminologie, begleitet von imposanter Gestik, stößt auf völliges Unverständnis, das sich auf dem naiv-neugierigen Gesicht der ratsuchenden Anita G. abzeichnet. 
Eine dritte Art der akademischen Rede ist dem snobistisch-fachidiotischen Vorlesungs-Typus entgegengesetzt. Die amüsante Geschwätzigkeit, die hier an den Tag gelegt wird, lässt sich im neudeutschen Jargon als Idealtyp des Infotainments verstehen. Der Vorlesungsstoff wird in möglichst breit umschreibenden und allgemeinverständlichen Formulierungen in stark verdünnter Dosis präsentiert. Eingestreut werden amüsante Geschichten und Plaudereien aus dem Nähkästchen, die

und unterhaltsam wirken sollen. Lernende fühlen sich oft unterfordert und durch die Zähigkeit der Rede zugleich überanstrengt. Der Verfasser erinnert sich an Vorlesungen, die der Historiker Hans Herzfeld in den 1950er Jahren an der FU Berlin hielt. Sein zweibändiges Buch „Die Moderne Welt 1789-1945“ (1959/60) hatte ein Vorlesungsskript zur Grundlage. Obwohl sorgfältig lektoriert und von unverdaulich-blumigen Schachtelsätzen befreit, vermittelt der Text immer noch einen Eindruck vom schleppenden Fluss einer Rede, die zugleich um Kurzweiligkeit bemüht ist. In einem Abschnitt, der zeigen soll, welche Konsequenzen die ,angelsächsischen Weltmächte" (Großbritannien und USA) aus dem 1. Weltkrieg zogen, verweist Herzfeld auf ihre Neigung, sich vom europäischen Schauplatz stärker zu entfernen. Auf Großbritannien bezogen fügt er dann hinzu: Es sei , in dieser Neigung durch die Einwirkung bestärkt worden, die die Entwicklung vom Empire zum Commonwealth hatte; anders als bei der vom Krieg niemals bis zur Grenze ihrer Kraft angespannten Union von Nordamerika, die auch im Rückfall zur Isolierungstradition die stärkste Wirtschaftsmacht der Epoche blieb, zeigten sich bei ihm schon unverkennbare Symptome, dass die natürlicherweise begrenzte Kraft des Mutterlandes nicht mehr genügte, um die seit 100 Jahren auf die Hegemonie zur See aufgebaute Weltmachtstellung in vollem Umfange zu wahren.“ (Herzfeld 1960, 2. Bd.: 277)

\section{Vorlesungs-Bewertung}

Das Verhältnis der Studierenden zum Vorlesungslernen ist zwiespältig. Zur Unerschütterlichkeit, die Vorlesungen bis heute bewiesen haben, passt einerseits, dass die Studierenden in die Hörsäle strömen, auch wenn es keine Prägibt und der Vorlesungsstoff in Lehrbüchern nachgelesen oder aus dem Internet heruntergeladen werden kann. Andererseits bröckelt der Vorlesungsbesuch erfahrungsgemäß ab. Am Anfang des Semesters sind viele da, am Schluss oft nur noch relativ wenige. Das mag teilweise mit anderen V tungen zusammenhängen. Jedoch fehlt es an der prinzipiellen Entschlossenheit, Vorlesungen als unverzichtbare Lerngelegenheiten zu nutzen.

Wie unsicher sich Studierende ihrer Aufnahmekapazität im Hörsaal sind, zeigt sich daran, dass zum einen ,wie verrückt" mitgeschrieben wird. Zum 
andern fällt es offenbar schwer, den Vortragenden zu folgen und gleichzeitig das Gehörte zu notieren. Aus dieser Unzufriedenheit mit dem Ergebnis des eigenen Mitschreibens ergibt sich der Wunsch nach Vorlesungsmitschriften. Diese wurden schon im 19. Jahrhundert eingefordert ${ }^{44}$ und werden auch noch im gegenwärtigen Vorlesungsbetrieb verlangt, obwohl es kaum einen Vorlesungsstoff gibt, der nicht in Büchern und speziell in den schon genannten vorlesungsadäquaten - Lehrbüchern nachgelesen werden kann. Das Misstrauen gegenüber der eigenen Aufnahmefähigkeit verbindet sich hier mit dem Verdacht, aus der Vorlesung weniger „,mitgenommen“ zu haben als zur konsolidierenden Nacharbeit notwendig erscheint. Jenseits solcher Unklarheiten gibt es über die lange Vorlesungstradition hinweg großes Lob.

\section{Vorlesungs-Lob}

Im Kern bezieht sich die positive Bewertung von Vorlesungen auf die fachliche Exzellenz und die Fähigkeit der Vortragenden, durch ihr Charisma zu faszinieren. Beides steckt lautmalerisch in der französischen Bezeichnung des cours magistral: Meisterhaft sowohl in der Demonstration von Kompetenz als auch in der Art des Vortrags. Solche Einschätzungen, die zumeist nicht in die Einzelheiten gehen, stecken in vielen sowohl historischen als auch aktuellen Berichten, in denen Studierende und ehemalige Studierende auf Vorlesungserlebnisse zurückblicken. Für das 19. Jahrhundert werden neben schon genannten Namen - herausragende Figuren wie Heinrich von Treitschke (Historiker), Max Weber und Lujo Brentano (Sozialwissenschaftler) gerühmt. Unter dem Eindruck der Vorlesung, die Letzterer im Sommersemester 1893 hielt, schreibt ein begeisterter Student der Nationalökonomie, Brentano sei „Gelehrter und Künstler“. In seinen Vorlesungen zur Wirtschaftsgeschichte habe sich ,seine Kunst ... bis zur Vollendung“ gesteigert (in: Ellwein 1997: 193). Auf einen weniger bekannten Juraprofessor (Amira, München) bezieht sich der spätere Bürgermeister von Bremen, Theodor Spitta: Nicht an Inhalten des im Wintersemester 1893/94 abgehaltenen Kollegs sei er interessiert gewesen. Angezogen habe ihn die „Gesamtpersönlichkeit, der Zauber, der von dem Menschen Amira“ ausgegangen sei (in: Ellwein 1997: 204).

Lebendige Erinnerungen von Studierenden des 20. Jahrhundert verbinden sich mit Philosophen wie Gadamer, Heidegger oder Jaspers. Auch wird

44 Vgl. etwa als Beispiel einer Vorlesungsmitschrift aus dem Wintersemester 1897/98: Hermann Diels, Griechische Philosophie, im Neudruck hrsg. von Johannes Salzwedel, 2010 . 

Tenor des ausgesprochenen Standard-Lobs fasst der Politologe Arnd Morkel in der Formulierung zusammen: „Wer einmal das Glück gehabt hat, einen bedeutenden Lehrer längere Zeit zu hören, weiß, dass der Gewinn einer guten Vorlesung nicht allein in dem besteht, was man schwarz auf weiß nach Hause tragen kann, sondern auch und vor allem darin, dass man einen Wissenschaftler , in seinem Denken, seinem Ernst, seiner Betroffenheit' (Zitat Jaspers) unmittelbar miterlebt." (Morkel 2000: 38) Das Vorlesungs-Lob ist demnach sowohl historisch als auch aktuell auf die idealtypischen Merkmale der Impuls-Vorlesung und formal darauf gerichtet, dass charismatische Funken auf die Zuhörenden überspringen.

\section{Vorlesungs-Kritik}

Was die Vorlesungskritik angeht, so schaffte die Studentenbewegung Ende der 1960er Jahre einen - wie sich später herausstellte - nur kurzlebigen Durchbruch. Ihre Kritik hatte zunächst eine Stoßrichtung, die hier nur am Rande interessieren kann. Hinter ihr stand ein verändertes Verständnis von Wissenschaft, die kritisch-rationaler und auf die gesellschaftspolitische Praxis bezogen sein sollte (Friedeburg u. a. 1968: 404ff.). In Projekten einer sogenannten „Kritischen Universität“ sollten die Studienordnungen inhaltlich grundlegend reformiert werden..$^{45}$

Soweit sich die Vorlesungskritik der Studentenbewegung auf Lernformen und speziell auf das Vorlesungsprinzip bezieht, so liegen ihr jene diffusen Unsicherheiten zugrunde, die schon als Ausgangspunkt des Vorlesungs-Lobs beschrieben wurden. Es überrascht daher nicht, dass es an präzisen Begründungen der Unzufriedenheit zumeist fehlt. ${ }^{46}$

Beim Besuch des Otto-Suhr-Instituts (OSI) der Berliner Freien Universität Ende der 1960er Jahre stach ein Plakat ins Auge, das am Eingang des Hörsaals angebracht war, in dem der Dozent Lodder seine Vorlesung abhielt. Auf dem Plakat stand in lakonischer Kürze: „Lodder ist Scheiße.“ Es ging ganz einfach darum, eine ganz plötzlich von fast allen Studierenden als „unmöglich“ empfundenes Lernformat abzuschaffen. Die vielfach verletzende Kritik richtete sich an die Adresse der Personen, die dieses praktizierten. So wandte sich etwa der Pädagogik-Student Oberlercher im „auditorium“, dem Sprachrohr des Asta-Hamburg, im November 1967 mit folgender Formulierung gegen den bruar 1968 eskalierte (Friedeburg u. a. 1968: 446ff.).

46 So Thieme 1982: 390f., der aus diesen Unklarheiten die Gründe für ein Scheitern der Hochschuldidaktik ableitet. Vgl. auch im Folgenden: Kap. III.2.2 
Pädagogik-Professor Wenke: „Zumutungen vom Schlage Wenke'scher Vorlesungen sind nur dadurch zu kritisieren, dass man sie sprengt. ${ }^{647}$

Aktuellere Berichte über Vorlesungs-Erfahrungen zeigen, dass sich das Unbehagen am Vorlesungsbetrieb erhalten hat. Repräsentativ ist zweifellos die Bewertung eines BWL-Studenten der Universität Ulm, nach der Vorlesungen in der Regel als langweilig empfunden werden und ihr Ertrag bezweifelt wird: „Mal wieder den inneren Schweinehund überwunden und trotz besseren Wissens in die BWL-Vorlesung marschiert? ... diesen Vormittag hätte man auch mit wesentlich nützlicheren Dingen verbringen können. Aber da muss man jetzt durch." Der Kommentator machte zudem einige Vorschläge zu Computer-Spielen, mit denen die Langeweile bekämpft werden kann. ${ }^{48} \mathrm{Als}$ es solche digitalen Möglichkeiten des Zeitvertreibs noch nicht gab, wurden beispielsweise Strichlisten angelegt, auf denen registriert wurde, wie oft die Vorlesenden, „Äh“ sagten.

Hinter solchen allgemeinen Signalen ungeduldiger Gereiztheit stehen zwei prinzipielle Ansätze der Vorlesungs-Kritik. Der eine betrifft Elemente snobistischer Fachidiotie. Abgelehnt werden professorale Selbstverliebtheit und fachverliebte Eigenbrötelei. Sie gehören zum Mythos von Wissenschaft, auf den bei der Beschreibung der allgemeinen Lern-Konstellation (Kap. III.1.) schon eingegangen wurde. Kritisiert wird ein Vokabular, mit dem Hörerinnen und Hörer als Normalsterbliche nicht klarkommen und das ihnen auf die Schnelle auch nicht verständlich gemacht werden kann. Diese Ablehnung eines an Esoterik grenzenden Vortragsstils fasst der Mainzer Philosoph Thomas Metzinger, der sich durch ein pointiertes Urteilsvermögen auszeichnet, exemplarisch zusammen. Er erinnert sich an die Erfahrungen, die er als lernbegieriger Student an der Goethe-Universität Frankfurt am Main gemacht hat. Ihm sei „bald der Verdacht“ gekommen, „dass es in diesen Vorlesungen und bei manchen geisteswissenschaftlichen Vorträgen überhaupt nicht um gelingende Kommunikation ging ..., sondern dass es sich hier um eine pseudointellektuelle Art von Angeberei und Drohverhalten handelte ..." (Metzinger 2010: 62).

Der zweite Ansatz der Kritik bezieht sich auf die Art und Weise, in der die herausfordernde Fülle des Vorlesungsstoffs bearbeitet wird. Kritisiert wird die Neigung von Vorlesenden, es in breit und langwierig angelegten Ausführungen an Präzision, aber nicht an Versuchen fehlen zu lassen, den Stoff unterhaltsam zu präsentieren. Dieser Ansatz gehört unter die idealtypische Rubrik des Info-

47 Der Spiegel, 52/1967 - Studenten Irre geworden/Hamburger Universität (Abdruck von Vorlesungs-Kritik des FU-Spiegels; ab 1966 veröff.).

48 Vgl. Studi-Spazz. Alles für Studierende in Ulm und Neu-Ulm, H.1/SS2007: 14; online: www.studispazz.de 
tainments. Exemplarisch formuliert es ein Brief des späteren Außenministers der Weimarer Republik, Walther Rathenau, Student des Maschinenbaus und der Elektrotechnik, an seinen Vater. Er ist von den Kollegien enttäuscht, die er 1889 am Münchner Polytechnikum besucht. Sie seien „so

und populär gehalten, als gelte es einen Handwerkerverein zu belehren“ (in: Ellwein 1997: 212) ${ }^{49}$ Hier wird eine auch aktuell immer noch monierte „Verdünnung" und Banalisierung der Stofffülle kritisiert. Es ist offensichtlich, dass die unterhaltsame Präsentation, zu der auch die eher peinliche Wiederholung längst bekannter Anekdoten gehört, das fachübergreifend bestehende studentische Bedürfnis missachtet, möglichst dichte und greifbare Informationen zu erhalten. Insoweit artikulieren sich in der Infotainment-Kritik auch Zweifel daran, dass der Anspruch, allgemeine oder spezialistische Instruktionen zu bieten, eingelöst wird.

Auch wenn sich der Frust der Lernenden nicht in Vorlesungskritik äußert, so bleibt er den Vorlesenden selten verborgen. Sie erleben ihn oft als eigene Lustlosigkeit („Steige mer halt wieder in die Bütt"). In der Erinnerung des Verfassers taucht der Tübinger Staatsrechtler Günter Dürig auf. Er ließ sein Lesepult einfach stehen, bewegte sich auf dem breiten Podest der Hörsaalfront nach links und dann wieder nach rechts, redete „herumtigernd“, um auf diese Weise mehr Schwung in seine Darbietung zu bringen. Andere Akteure sprechen ihre Hörerinnen und Hörer an, stellen ihnen Fragen und machen ins Publikum. ${ }^{50}$ Sie folgen auf diese Weise dem Vorbild von Schauspielerinnen und Schauspielern, die Kontakt zu ihren Zuhörenden suchen.

Dabei handelt es sich um Vorlesende mit charismatischer Ambition, die ihre Wirkung auf das Publikum zu steigern und auf diese Weise ihre Selbstzweifel zu überwinden suchen. In die Reihe dieser professoralen Stars gehört auch der schon genannte Michel Foucault, dessen Vorlesungen bekanntlich großen Zulauf hatten. Er machte wiederholt geltend, dass er unter fehlendem Kontakt zu seinem Publikum - das heißt unter Fernkommunikation - leide. Bei seinen Kontaktversuchen, vor allem über Fragen an seine Hörerinnen und Hörer, scheiterte er aber immer wieder. Als Vorlesender habe er sich dem Gefühl „totaler Einsamkeit“ (,solitude totale“) nie entziehen können. ${ }^{51}$ Sowohl

49 Nebenbei zeigt sich hier, dass an den Technischen Hochschulen, die im Zuge der Zweiteilung des deutschen Bildungssystems seit Beginn des 19. Jahrhunderts entstanden (vgl. Ellwein 1997: 122ff.) Vorlesungen als zentrale Lernform übernommen wurden.

50 Dies wird beispielsweise von Vorlesungen des Münchner Soziologen Armin Nassehi berichtet.

51 Einschlägige Äußerungen Foucaults wurden von einem Journalisten des Nouvel Observateur (Gérard Petitjean, „Les Grands Prêtres de l'université française“, 7. April 1975) aufgezeichnet und in einer Einführung (Avertissement) zit. in: Foucault 1999: VIII. 
im Falle von Foucault als auch im Blick auf andere Vorlesende belegt diese Einsamkeit keineswegs eine fehlende charismatische Ausstrahlung. Vielmehr kommt zum Ausdruck, dass überzeugende Lernformen nicht nur von den Lehrpersonen - zum Beispiel von der Person des „Zauberers“ Foucault oder der des „unattraktiven“ Vorlesers Lodder - abhängig sind.

\section{Vorlesungen als Infotainment}

Es gibt Lehrpersonen, die unter studentischer Unzufriedenheit leiden und durch Infotainment, wie es in der Vorlesungs-Kritik schon angedeutet wurde, gegensteuern wollen. Als normatives Modell, an das Vorlesungen vergleichend angenähert werden können, bieten sich Reden an, die hier in dem Sinne Catchall-Reden $^{52}$ genannt werden, dass Menschen sowohl unterschiedlichen Alters und Geschlechts als auch unterschiedlicher sozialer und Herkunft unterhalten werden sollen.

$\mathrm{Zu}$ den Catch-all-Reden gehören Ansprachen, die auf kleineren oder größeren Geburtstagen gehalten werden und zu den Happy-Birthday-Gesängen passen, die bei solchen Anlässen fast unvermeidlich sind. Gemeint sind auch Reden, die von Verbänden und Unternehmen unterschiedlichsten Typs arrangiert werden, um anlässlich von Jahrestagen und Jubiläen ihre Corporate Identity zu festigen. Welches sind die Kennzeichen solcher unakademischen Reden, die stark auf Infotainment ausgerichtet sind?

Ein allgemeines Charakteristikum von Catch-all-Reden besteht darin, dass sie, wie es umgangssprachlich heißt, launig, also humorvoll sind. Das bedeutet beispielsweise für die feierliche Rückblicks-Rede, die anlässlich der Gründung eines Verbandes gehalten wird, dass aus der Distanz merkwürdig anmutende Kinderkrankheiten des Anfangs, über die amüsant berichtet wird, eher verhaltenes Schmunzeln und Lachen auslösen. Ein Autor, der selbst Catch-all-Reden geschrieben und über viele Jahre einen Reden-Beratungs-Dienst herausgegeben hat, fasst seine Tipps zur gelungenen Rede in 10 Punkten zusammen. ${ }^{53}$

52 Der Begriff orientiert sich am „Catch all“-Gedanken, den der Politikwissenschaftler Otto Kirchheimer auf Parteien anwandte („Catch-all-parties“; deutsch: „Allerweltsparteien“), die mit ihrer Programmatik darauf zielen, Wähler unterschiedlichster sozialer Herkunft zu gewinnen (Kirchheimer 1965: 24-41). Sowohl unakademische Reden vom Catch-all-Typ als auch akademische zielen auf Neuronengruppen, die sich im vorderen Stirnhirn (Broca-Gebiet), im oberen Schläfenlappen (Wernicke-Gebiet) und nach neueren Erkenntnissen auch im unteren Schläfenlappen (Brodmann-Areale 20/37) befinden (vgl. Rösler 2011: 348).

53 Burkhard Strack, Die zehn Gebote für den Redner (12.6.2008); online verfügbar unter: https://www.experto.de/kommunikation/reden/die-zehn-gebote-fuer-den-redner.html (zuletzt geprüft 10.7.2018). 
Seine wichtigsten Tipps lauten:

Catch-all-Reden müssen sowohl den Intellekt ansprechen als auch zu Herzen gehen. Deshalb erfordern sie einerseits sprachliche Klarheit, Deutlichkeit und Richtigkeit. Um die Gefühle des Publikums zu erreichen, müssen Reden andererseits auf dessen „Sympathien, Erwartungen, Befürchtungen, Vorurteile oder Fragen" abgestimmt sein. In angemessener Tonlage und begleitet von Mimik und Gestik muss anschaulich und in aussagekräftigen Bildern formuliert werden.

Das betrifft beispielsweise Geburtstagsreden, die in dem Sinne auch Kompetenz erfordern, dass Rednerinnen und Redner mit dem Lebenslauf eines Geburtstagskinds vertraut sind. Indessen kommt es nicht auf die Berücksichtigung von Einzelheiten an. Dasselbe gilt für eine Rede, die etwa beim Jubiläum eines Unternehmens zum Thema „Die Freiheit des Unternehmertums“" gehalten werden könnte. Da müssten nicht alle möglichen Freiheitsbegriffe durchgenommen und historisch eingeordnet werden. Vielmehr komme es darauf an, den Freiheitsgedanken mit speziellen unternehmerischen Eigenschaften und ihrem erfolgreichen Einsatz zu verbinden. Damit wird indirekt auch das „Hauptgebot“ gelingender Rede berührt, das alle anderen Tipps übergreift: Reden müssen kurz und bündig sein, nach der Devise „Rede weniger, sage mehr!“”.

Versucht man die Merkmale von Catch-all-Reden auf Vorlesungen zu übertragen, so lässt sich von den beschriebenen Vorlesungs-Idealtypen her ein Mischtyp zusammensetzen, über den das Folgende gesagt werden kann:

Indem klare und deutliche Botschaften unterhaltsam ausgeschmückt (=Idealtyp Infotainment) und in anschaulich-bildhaften Formulierungen (=Verzicht auf die idealtypische Komponente snobistische Fachidiotie) dargeboten werden, können Vorlesungen an Verdaulichkeit gewinnen. Zur Annäherung von Vorlesungen an das Modell von Catch-all-Reden würde auch ein gewisses $\mathrm{Ma}$ an Charisma, jedoch keinesfalls perfektes Charisma der Vorlesenden gehören. Es ginge zum einen um ihr Bemühen, eine angemessene Tonlage zu die sich nicht vom anschaulichen Vortragsstil trennen lässt (= charismatischer Idealtyp/rhetorische Seite). Zum anderen müsste die Aufforderung eingelöst werden, Mimik und Gestik einzusetzen (=charismatischer Idealtyp/ persönliche Ausstrahlung).

Mit einer in dieser Weise dosierten Ausgestaltung der Infotainment-Komponente können sich Vorlesungen möglicherweise einer Vorlesungs-Kritik entziehen, wie sie oben illustriert wurde. Es bleibt aber immer noch die Frage zu beantworten, inwieweit sie mit einer solchen Akzentuierung von Infotainment zur Wissensvermittlung beitragen können. Zuvor muss in aller Kürze darauf eingegangen werden, wie selbstverständlich (Massen-)Vorlesungen abgehalten werden. Ihr Instruktions-Anspruch lässt sich dann mit neurobiologischer Begründung zurückweisen. 


\section{Lob von (Massen-) Vorlesungen als Instruktions-Vorlesungen}

(Massen-)Vorlesungen (im Folgenden nur: Vorlesungen) werden nicht nur von Routiniers des Hochschulbetriebs, sondern auch von Hochschuldidaktikerinnen und -didaktikern als ebenso unausweichliches wie unentbehrliches ,didaktisches Mittel der Informationsvermittlung“" angesehen (Metz-Göckel: 75). Dabei wird implizit und teilweise auch explizit unterstellt, dass Vorlesende sich um möglichst wenig Fachidiotie, um dosiertes Infotainment und um ein Mindestmaß an charismatischer Präsentation bemühen. Sind diese Kriterien erfüllt, steht der Anspruch von Vorlesungen, der allgemeinen und spezialistischen Instruktion zu dienen, so gut wie nicht in Frage. Alle in der beschriebenen Vorlesungs-Kritik enthaltenen Zweifel am Instruktionswert frontaler Rede werden dabei übergangen.

Für den Hochschuldidaktiker Hans-Peter Voss stellen Vorlesungen „den ,State of the Art' ihres Faches“ vor. Durch sie werde „,der Kanon des Wissens

und elaboriert, der von dem späteren Absolventen erwartet wird“54. Entsprechend hält Adi Winteler in einem mehrfach aufgelegten Praxisbuch Vorlesungen für ,gerechtfertigt, wenn ein

Wissensgebiet oder eine Fertigkeit unterrichtet wird, die alle Studierenden beherrschen müssen, wenn der Hauptzweck die Informationsvermittlung ist" (Winteler 2011: 129). Dabei neigt Winteler ebenso wie der Hochschuldidaktiker Hans Jürgen Apel dazu, die dem Lob für Impuls-Vorlesungen zugrundliegende Annahme, Vorlesende brillierten durch charismatisch-didaktisch fundierte Kompetenz, auf die Instruktions-Vorlesungen zu übertragen. Der Gedanke ist: Wer - im Sinne von Impuls-Vorlesungen - zum Nachdenken und zur Aufmerksamkeit für Zusammenhänge mobilisieren kann, ist auch in der Lage, Wissen zugänglich zu machen (Apel 1999: bes. 8; 48; 69f.).

Auch Studierende und ihre Vertretungen glauben das. Sie erwarten, dass Vorlesungen eine Menge mühevoller Sucharbeit und besonders auch anstrengende und zeitaufwendige Lektüre ersparen können, wenn in Einführungs- und Überblicksvorlesungen - auch im Blick auf den Prüfungsstoff - verlässlich gezeigt wird, was man unbedingt wissen muss. ${ }^{55}$

Besonders auffällig ist, dass das Vorlesungsformat unter jüngeren Wissenschaftlern, die für eine im Aufschwung Hochschuldidaktik stehen, so gut wie nicht problematisiert wird. Auf einer Tagung der Spring School for Academic Staff Developers, die vom 7.-9. März 2012 am Hoch-

54 In: Neues Handbuch Hochschullehre, Berlin 2006: E 2.1,3.

55 Diese Feststellungen beruhen auf Erfahrungen, die der Verf. in Curriculum-Gremien (1970er Jahre bis heute) gemacht hat. 
schulzentrum der TU Dortmund stattfand, war die Vorlesungsproblematik nur ein Thema am Rande. Anhand eines Posters wurden die „Herausforderungen an didaktisches Handeln in großen Vorlesungen“"im Schwerpunkt als Problem der Lehrpersonen abgehandelt. Befragungen eines BMBF-Projekts ergaben, dass hohe Belastungen durch Vorlesungen zu Erscheinungen von Resignation und sogar zum Burnout führen. Bezeichnend ist, dass keine Fragen nach dem Instruktionseffekt von Vorlesungen gestellt wurden. Im Besonderen blieb unbedacht, dass Instruktions-Vorlesungen und speziell Massenvorlesungen sich nicht mit der seit einigen Jahren vielfach beschworenen Maxime vertragen, nach der es für hochschuldidaktisch geschulte Lehrpersonen darauf ankomme, einen ,Shift from teaching to learning“ zu vollziehen (Welbers u. a.: 2005; vgl. Kap. V.2.).

Nun ist durchaus einzuräumen, dass es besonders begabten Lehrpersonen, wie sie in der Erinnerung an denkwürdige Vorlesungserlebnisse „hervorgekramt" werden können, dank außerordentlicher Fähigkeiten gelingen kann, durch ihren frontalen Vortrag massiv zur Wissensvermittlung beizutragen. Die in der Vorlesungs-Kritik enthaltenen Zweifel daran, dass dies durchschnittlich begabten Vorlesenden möglich ist, werden durch neurobiologische Erkenntnisse gestützt. Sie bauen auf den neurobiologischen Grundlagen ertragreichen Lernens auf, wie sie im II. Kapitel skizziert wurden. Zunächst wird das mangelhafte Vorlesungslernen auf die beschränkten Kapazitäten des Kurzzeitgedächtnisses bezogen. Es zeigt sich aber auch, dass es Vorlesungen an emotional-motivationalen Schubkräften fehlt.

\section{Neurobiologisch informierte Argumente gegen den Instruktions-Anspruch von (Massen-)Vorlesungen}

Vorlesungsbesuche sind mit hohem Energie- und Zeitaufwand verbunden. Man muss oft früh aufstehen und eine lange Anreise auf sich nehmen. Die Studierenden werden manchmal nur informell dazu angehalten, manchmal aber auch durch Studienordnungen formell dazu

Vorlesungen $\mathrm{zu}$ hören. Ob freiwillig oder unfreiwillig, begeben sie sich in der erklärten Absicht in Hörsäle, Wissen allgemeiner und

Art zu erwerben, das sie für Zwecke einer Überprüfung durch Klausuren und Examina und als Ausweis ihrer Hochschulausbildung brauchen.

\section{Mangelnde Aufnahme- und Haltefähigkeit des Kurzzeitgedächtnisses}

Zur Kennzeichnung der Verarbeitung von Informationen im Gehirn hat der Autor B. J. Baars die Metapher des neuronalen Arbeitsplatzes (,workspace“) 
eingeführt. Zunächst fragt es sich, was im Hörsaal überhaupt auf dem Arbeitsplatz und was speziell in dem Teilbereich ankommt, der - in einer künstlichen Abgrenzung vom sogenannten Langzeitgedächtnis - Kurzzeitgedächtnis genannt wird. Für die akustische Informationsaufnahme, auf die es im Hörsaal vor allem ankommt, gilt die erwähnte Beschränkung von , $7+/-2$ items“ pro Intervall des Vortrags. Diese ohnehin schon geringe Aufnahmekapazität reduziert sich zusätzlich, wenn ein „Effortmechanismus“ in Gang gesetzt wird, der im Hörsaal darin besteht, dass das Gehörte in Buchstaben, Wörter und Sätze umgewandelt wird und diese - unter Nutzung der motorischen Areale - niedergeschrieben werden. Das liegt daran, dass die in Gehirnen verfügbare Energie auf die einzelnen Komponenten der Informationsverarbeitung aufgeteilt wird. Wer hört, ohne mitzuschreiben, hört mehr. Wer mitschreibt, hört weniger.

Es sind diese bei der Informationsaufnahme im Hörsaal, die mit kombiniert sind, die darauf beruhen, dass in der Lern-Konstellation des Hörsaals das Potential des neuronalen Arbeitsplatzes nur unvollkommen ausgeschöpft werden kann. Dies lässt sich durch einen Kontrastvergleich mit der Lern-Konstellation des Schachspiels illustrieren, wenn dieses als Simultanschach organisiert wird. Dabei ist es bei diesem Vergleich unerheblich, ob es - wie im Schach - um prozedurale Gedächtnisbildung oder ob es - wie im Hörsaal - in erster Linie darum geht, dass semantisches Wissen übermittelt werden soll.

Es messen sich Schach(groß)meister mit einer kleineren oder auch größeren Zahl von Gegnern, die an je speziellen Brettern sitzen und vom Meister lernen wollen. Der Meister und die Schülerinnen und Schüler bewegen ihre Figuren auf dem Schachbrett in Übereinstimmung mit dem Know-how, das sie auf ihrem neuronalen Arbeitsplatz zum Spiel mitbringen.

Parallel zur Lern-Konstellation des Simultanschachs begegnen sich im Hörsaal Vorlesende und Studierende. Gestützt auf ihren mit hoher Kompetenz ausgestatteten neuronalen Arbeitsplatz, liefern Vorlesende Informationen, die auf den noch wenig kompetenten neuronalen Arbeitsplatz der Studierenden übertragen werden sollen. Dabei ist der entscheidende Unterschied zur Konstellation des Schachspiels, dass die Vorlesenden ,von oben herab spielen“, wogegen die Meister mit ihren Schülerinnen und Schülern auf demselben Brett auf gleicher Höhe - spielen.

Die Bedeutung dieser asymmetrischen Lern-Konstellation im Hörsaal wird später noch besonders hervorgehoben. Bei der hier zunächst interessierenden Frage, wie groß die Chance ist, dass der im Hörsaal vorgetragene Stoff über das Kurzzeitgedächtnis im Langzeitgedächtnis der Lernenden landet, geht es aber um Folgendes:

Im Unterschied zu den Vorlesenden, die ein Hör-Kollektiv ansprechen, bewegen sich die Meister beim Simultan-Schach von Brett zu Brett, von einem 
Lern-Individuum zum anderen. Dabei ist wichtig, dass die am Schachbrett Lernenden für ihre Verarbeitung der Züge der Meister immer Bedenkzeit haben. Sie ist so lang, wie die Meister für die Züge bei den jeweils anderen Brettern und Partien brauchen.

Demgegenüber gibt es am neuronalen Arbeitsplatz der im Hörsaal Lernenden nichts auszutüfteln und auszudenken. Es fehlt an massiver Erregung der elektro-chemischen Schleifen, die im Stirnhirn für die Informationsaufnahme zuständig sind. Die Informationen, die im Intervall von 1-3 Sekunden ins Stirnhirn der Lernenden einströmen, werden - wie beschrieben - nicht nur aufgrund fehlender inhaltlicher Aufnahmefähigkeit des Kurzzeitgedächtnisses nur in geringer Dosis aufgenommen. Der Verarbeitungsverlust wird vielmehr dadurch noch beträchtlich verstärkt, dass die Lernenden - ohne Bedenkzeit dem ununterbrochenen der Vorlesenden ausgesetzt sind. Die Informationen unaufhaltsam nach und gehen - nur teilweise oder gar nicht bearbeitet - schon auf der Stufe des Kurzzeitgedächtnisses verloren.

Die beschränkte zeitlich-inhaltliche Aufnahmefähigkeit des Arbeitsgedächtnisses, die in Modulen angelegt ist, gilt prinzipiell für alle Modalitäten (Auge, Gehör ...) und Inhaltsarten (Namen, Gesichter ...), im Besonderen aber für Zahlen. Beim Test der Fähigkeit, Telefonnummern aufzunehmen, gehen Nummern verloren, die in kurzem Abstand an zweiter und dritter Stelle dargeboten werden (Roth 2011: 141). Das gilt auch und im Besonderen für Folien, die sich in Power-Point-Darbietungen oft jagen. Ich nehme einen Beispielsbereich, der einigermaßen aufmerksamen Zeitungsleserinnen und -lesern geist. Werden in Vorlesungen Wahlergebnisse unterschiedlicher Zeitpunkte und unterschiedlicher Länder hintereinandergeschaltet, so ist die angestrebte Gegenüberstellung nicht möglich, weil die Lernenden dem dargebotenen Zahlenmaterial sehr bald nicht mehr folgen können. Das gilt erst recht, wenn zusätzliche Materialien vorgestellt werden. Möglicherweise werden aktuelle Wahlergebnisse auf die Sozialstruktur der Wählerschaft (Alter, Geschlecht, Herkunft ...) bezogen. Nach wenigen Folien haben die Vorlesenden, oft ohne es zu merken, den Kontakt zu ihren Hörerinnen und Hörern verloren.

Im II. Kapitel ließ sich einerseits zeigen, dass die im Kurzzeitgedächtnis in zeitlich-inhaltlicher Beschränkung - aufgenommenen Informationen neu sein müssen. Andererseits ist deren erfolgreiche Einlagerung im Langzeitgedächtnis davon abhängig, dass sie an schon vorhandene Informationsbestände anknüpfen können. An wenig Bekanntes kann immer nur wenig Neues angehängt werden. Fehlt es völlig an Vorkenntnissen, so kommt das von Fachleuten vorgetragene und synthetisierte Wissen bei den Lernenden nicht an. Das merken beispielsweise Studierende der Geschichte, die sich noch nicht mit der französischen Revolution beschäftigt haben und daher eine noch so kompetent vorgetragene Schilderung und

Einordnung des Stoffes nicht im 
Gedächtnis behalten können. In derselben Lage

sich Studierende der Physik und Mathematik, von denen immer wieder zu hören ist, in ihren ersten Vorlesungen - fern vom vertrauten Schulstoff - hätten sie ,überhaupt nichts verstanden“. Insoweit missachtet das oben beschriebene Vorlesungs-Lob die Voraussetzungen, die erfüllt sein müssen, wenn Vorlesungen sein sollen. Ihr Anspruch, in großer Breite Wissensfelder zu eröffnen, die den Studierenden bislang nicht zugänglich waren, geht in die Irre.

Auch wenn es nicht zum völligen Leerlauf des Vorlesungslernens kommt, ist doch unverkennbar, dass zwischen der Fülle des vorgetragenen Stoffes und der Spärlichkeit des hängenbleibenden Wissens eine Diskrepanz besteht. Wie groß diese ist, hängt davon ab, inwieweit in der Aufnahme, Weitergabe und Aufbewahrung von Informationen dadurch zumindest abgeschwächt werden, dass in den Netzwerken zwischen Arbeitsgedächtnis und Langzeitgedächtnis emotionale Schubkräfte wirksam sind. Fehlen sie, so werden die Vorlesungslernens bekräftigt.

\section{Mangelnde emotional-motivationale Schubkräfte}

Um den Lernertrag des Vorlesungsprinzips hinsichtlich der emotionalen Schubkräfte von Lernvorgängen zu überprüfen, muss die vertikale Beziehung der Lernenden zu den Lehrpersonen mit deren horizontaler Beziehung zu Mit-Lernenden kombiniert werden. Im Folgenden wird immer zuerst die

\section{Mangelndes Attachment:}

Im Hörsaal thronen die Lehrpersonen sehr weit über den Lernenden. Auf diese asymmetrische Konstellation wurde schon hingewiesen. Auch in eher intimen Veranstaltungsräumen der Traditionsuniversität, in denen die Lehrpersonen eine überschaubare Zahl von Lernenden trafen, wurde auf gehörigen Abstand Wert gelegt, zumeist betont durch ein erhöhtes Vorlesungspult. Nicht nur in den zahlenmäßig gering besetzten Orchideenfächern wird dieser Abstand heute noch eingehalten. Auch wenn in aktuell gegründeten Einrichtungen, in denen Eliten ausgebildet werden sollen, sich Lehrpersonen und Lernende im Hörsaal auf einer Ebene begegnen, so steht die klare Trennung zwischen denen, die hören, und denen, die vorlesen, immer noch außer Zweifel. Das aber heißt, dass, vermittelt über den Vorlesungsbetrieb, enge emotionale Bindungen weder entstehen noch - für den Fall, dass sie durch soziale Kontakte außerhalb des Lernbetriebs entstanden sind und weiter entwickelt werden. Damit fehlt es in der Konstellation von Vorlesungen an Voraussetzungen dafür, dass der zwischen Arbeitsgedächtnis und Langzeitgedächtnis gefördert wird. 


\section{Mangelnde empathische Resonanz:}

Wenn es stimmt, dass das beispielhaft dargestellte Vorlesungs-Lob einer Ausnahme-Konstellation gilt und die Vorlesungs-Kritik, wie sie in der Nachfolge der Studentenbewegung geübt wird, die unbefriedigenden Lernprozesse in Hörsälen in der Tendenz zutreffend wiedergibt, so bedeutet das für den abweichenden Fall das Folgende:

Lernende sind vom Einfallsreichtum, von der rhetorischen Brillanz und von der bilderreichen Sprache einer Vorlesung in Bann gezogen. Es macht ihnen keine Mühe, sich in die Gefühlswelt der Vorlesenden hineinzuversetzen. Für Lernende ist nicht schwer nachvollziehbar, dass Rednerinnen und Redner an Auftritten, die ihnen leicht „von der Zunge gehen“, in ihrem Innern großen Gefallen Umgekehrt erkennen Vorlesende auch aus der Ferne, dass Lernende, an ihren Lippen hängend, in ihrem Gefühlsinneren Beifall spenden, auch wenn sie diesen nach außen hin nicht lautstark kundtun. Parallel dazu kommt es in einer solchen Ausnahmekonstellation dazu, dass sich Lernende und Mit-Lernende im Austausch von gestischen Signalen und Augenbewegungen, fast stillschweigend, darüber einig sind, dass sie die ihnen gebotene Rede zustimmend aufnehmen und genießen. Zwischen allen am Vorlesungs-Event Beteiligten bestehen insoweit ebenso lebendige wie präzise Empathie-Beziehungen.

Einer solchen Ausnahmelage steht indessen eine Regelkonstellation diffuser Empathie gegenüber. Sie ist dadurch gekennzeichnet, dass unter fast allen Beteiligten der innere Eindruck vorwiegt, nicht nur räumlich weit voneinander entfernt zu sein. Der in der Regel eher gemächliche der Lehrpersonen bietet für Lernende kaum klaren Aufschluss darüber, ob die Vorlesenden eher mit Konzentration oder aber eher mit Unlust bei der Sache sind. Soweit es im Fluss der Rede leicht abgestufte Geschwindigkeiten und leicht wechselnde Tonlagen gibt, geht aus ihnen für Lernende kaum hervor, ob Lehrpersonen engagiert vortragen oder lediglich darum bemüht sind, sich selbst bei Laune zu halten.

Den Lehrpersonen geben schweigendes Mitschreiben sowie punktuelle Regungen und Geräusche im Publikum nur unzureichend Auskunft darüber, ob die Lernenden dem Vortrag eher mit Zustimmung und Verständnis folgen

Dieselbe diffuse Empathie bestimmt auch das gegenseitige Verhältnis der Lernenden. Was fühlen Nachbarinnen und Nachbarn? Wie ist der gemeinsame, nahezu stumme Mitvollzug der dargebotenen Rede zu interpretieren, eingetaucht in eine spannungslose Geräuschkulisse und nur verhalten begleitet von körperlicher Gestik?

\section{Mangelnde Nähe im Rollenspiel der Vorlesungen:}

Eduard Baumgarten, der in den 1950er und 1960er Jahren in Mannheim Sozialwissenschaften lehrte, gehörte zu den wenigen Professoren, die über die 
eigene Profession, speziell über professorale Grundeinstellungen und darüber nachdachten, welche Konsequenzen diese für das Lernen an der Universität haben. Was Vorlesungen angeht, so verband er mit ihnen eine bestimmte Art von „Genuss“, die besonders im „Massenkolleg“ zu ,eigentümlich rauschhafter Steigerung des Personengefühls von Rednern vor Massen" führe (Baumgarten 1963: 8). In Übereinstimmung mit solchen Einschätzungen gelten Vorlesungen als zentrale Herausforderung des universitären Berufsfelds. Nicht alle Akteure dürfen Vorlesungen halten. Wer ein herausgehobenes Amt möchte, muss den „Vorlesungstest“ (Antrittsvorlesung) bestehen. Wer das Amt verlässt, hält in der Regel immer noch eine Abschiedsvorlesung. Insistierende Befragungen von Professorinnen zeigen, dass diese sich ihrer V ebenso arbeitsaufwändige wie kräfteraubende Vorlesungen zu halten, in der Einstellung unterziehen, als Frauen ihre (immer noch bezweifelte)

Eignung beweisen zu müssen. Für viele Hochschullehrerinnen und Hochschullehrer stehen Vorlesungen nicht nur im Mittelpunkt ihrer Berufstätigkeit, sondern ihrer ganzen Existenz. Das gilt selbst für Wissenschaftler, die ihr persönliches und Leben sehr stark auf enge mitmenschliche Beziehungen und Team-Arbeit aufgebaut haben. ${ }^{56}$

Vorlesungskritik, wie sie beschrieben wurde, ganz zu schweigen von der im Umfeld der Studentenbewegung vorkommenden Sprengung von Vorlesungen, wird als existentielle Niederlage erfahren. Vor dem Hintergrund transnationaler Traditionen gilt dies auch für andere Länder, zum Beispiel für Frankreich. Pierre Bourdieu hat in seinen Untersuchungen zum Homo academicus die studentischen Streiks im französischen Mai 1968 aus der Sicht der Lehrpersonen als „Barbarei“" beschrieben, die sich gegen die Vorlesung (le cours) als ,,instance de grace“" gerichtet habe (Bourdieu 1984: 238f.).

Wenn demnach Vorlesende etwas Heiligenhaftes haben, so wird dadurch die Distanz zum ,gemeinen“ Publikum besonders groß und offensichtlich. Schon die in jedem Fall bestehende räumliche Entfernung, in der sich von oben herab Lehrpersonen gegenüber weit unten Zuhörenden lässt Letztere unwichtig erscheinen. Für den Soziologen Baumgarten hat diese Asymmetrie, die sich besonders im „Massenkolleg“ zeigt, fatale Folgen: Sie „verdirbt auf beiden Seiten die innere Diskussionsbereitschaft und Diskussionsfähigkeit, zerstört im Denkprozess dessen dialogische Form.“ (Baumgarten 1963: 8f.)

56 Beobachten lässt sich dies beispielsweise bei dem Neurowissenschaftler Ernst Pöppel, der seine letzte Vorlesung als tiefen Einschnitt seines Forscherlebens versteht. (Vgl. ders., ,,Von der letzten Vorlesung zum interkultuellen Dialog“, in: Ders./Beatrice Wagner 2010: 226ff.) 
Diese Behauptungen können in die Sprache des Theaters und ins Rollenspiel auf der Bühne des Theaters übersetzt werden. Zugleich lässt sich fragen, wie Vorlese-Inszenierungen in Hörsälen neurobiologisch fundiert sind. Dabei zeigt sich ein Widerspruch zwischen den von Baumgarten behaupteten Hochgefühlen der Vorlesenden und dem traditionellen Anspruch, den Studierenden Wissen zu vermitteln.

Es wurde gezeigt, dass das Rollenspiel sowohl in der frühkindlichen Erziehung als auch bei Erwachsenen emotionale Schubkräfte freisetzen kann. Sie fördern den Prozess ertragreichen Lernens, wenn die spielenden Personen nah beisammen sind. Unter dieser Bedingung wird es im Spiel möglich, die in engen affektiven und empathischen Verbindungen steckenden Kräfte und die aus der Aussicht auf Belohnung kommenden Antriebe zu addieren. Wenn auf diese Weise die inhaltlich und zeitlich begrenzte Fähigkeit des Kurzzeitgedächtnisses gestärkt wird, Lernimpulse zum Langzeitgedächtnis weiterzuleiten, so gilt umgekehrt das genaue Gegenteil:

Die Asymmetrie im Rollenspiel der Vorlesung besteht in der Dominanz der Vorlesenden-Rolle. Alle tragenden Rollen, auch die des fragenden Zweiflers, sind in einer Person konzentriert. Die Hörenden werden in die Rolle von „stummen Bäumen“ gedrängt. Wer diese Rolle spielt, hat keine Möglichkeiten, seine Talente unter Beweis zu stellen und dabei sich selbst und andere zu überraschen. Es fehlt an Belohnungen, die, wie gezeigt wurde, in Überraschungseffekten stecken. Da fehlende Belohnungen beim Publikum selbst wiederum auf die Belohnungsstrukturen der Vorlesenden zurückwirken, wird einsichtig, warum selbst Charismatiker wie Foucault und andere ihre Inszenierungen als unzulänglich erleben. Wie viel mehr gilt das dann für die Regelkonstellation von Vorlesungen, in der es bei den Lehrpersonen nur mit großen Abstrichen Hochgefühle geben kann. Dasselbe gilt für die Lernenden.

Was darüber hinaus die horizontalen Beziehungen zwischen ihnen und den Mit-Lernenden angeht, so sind auch hier das Attachment und die empathischen Beziehungen in der Regelkonstellation von Vorlesungen unterentwickelt. Zwar gibt es durchaus Bemühungen um Gemeinschaftserlebnisse: Einzelne Studierende, die sich kennen, sitzen nebeneinander, zuweilen sogar auf dem Boden oder auf Plätzen, die sie sich gegenseitig freihalten. Im Verlauf von Vorlesungen kommt es zum - freilich eher zurückhaltenden - Austausch von Gesten, zu Blickwechseln, zuweilen auch zu Flüster-Kontakten. Lacher, die von Hauptrollenspielenden provoziert werden, sind selten. Das aus Erlebnissen der Gemeinsamkeit kommende Wohlgefühl bleibt daher auf einem niedrigen Niveau der Erregung.

Im Ganzen führt die asymmetrische Rollen-Konstellation von Vorlesungen dazu, dass die Gefühlslage in Hörsälen in der Regel gedämpft bleibt. Die allgemeine Stimmung ist am deutlichsten im routinemäßigen Klopfbeifall greifbar, 
der am Schluss von Vorlesungen auch im besten Falle kaum aufbraust. Das alles bedeutet, dass es an günstigen emotionalen Voraussetzungen für ertragreiches Lernen fehlt.

Bei den Lernvorgängen, die sich in Hörsälen abspielen, handelt es sich um Lernen in Fernkommunikation. Die Lernenden sind weit weg von den Lehrpersonen. Auch ist die Entfernung zu den Mit-Lernenden in dem Sinne groß, dass die in „kollektiver Intentionalität“ Zuhörenden nur spärliche Kontakte zueinander haben. Neurobiologisch heißt das, dass es sowohl in der Vertikalen als auch in der Horizontalen nur geringes Attachment, geringe empathische Resonanz und im Rollenspiel der einseitig von oben diktierten Spielzüge wenig Ungewissheit und daher geringe Aussichten auf emotionalisierende Belohnungserfahrungen gibt. Es fehlen „emotionale Kaskaden“ (Damasio) und damit Voraussetzungen dafür, dass sich aus dem schmalen Angebot des ,aufnahmefaulen“ Kurzzeitgedächtnisses für das Langzeitgedächtnis ein Optimum herausholen lässt.

Vereinfacht gesagt sind Vorlesungen unbefriedigend, weil in der Fernkommunikation zwischen Vorlesenden und Lernenden zu wenig passiert. In neurobiologischer Perspektive heißt das: Der Erregungsgrad in den zuständigen neuronalen Netzen des Lernapparats Gehirn ist nicht groß genug. Es fehlt an emotional-motivationalen Stützen, die für ertragreiches Lernen unverzichtbar sind.

\section{Zusammenfassung}

Überprüft man zusammenfassend die Ansprüche, die mit dem Vorlesungsprinzip verbunden werden, so stellt eine neurobiologische Überprüfung zuallererst das Verständnis von Vorlesungen, speziell von Massenvorlesungen als Instruktions-Vorlesungen, in Frage. Es zeigt sich eine offensichtliche Diskrepanz zwischen der Fülle des vorgetragenen Stoffes und der Spärlichkeit des hängenbleibenden Wissens. Das hat zunächst und vor allem mit der geschilderten „Aufnahmefaulheit“" des Kurzzeitgedächtnisses zu tun. Diese führt dazu, dass ein beträchtlicher Teil des vorgetragenen Überblicksstoffs verloren geht. Hinzu kommt, dass die relativ wenigen Informationen, die über die Eingangshürden kommen und sich nur kurze Zeit halten lassen, nur unter besonderen Voraussetzungen ins Langzeitgedächtnis weiterbefördert werden: Die große Unzulänglichkeit instruktionsbezogener Vorlesungen besteht darin, dass sie in Fernkommunikation ablaufen und keine engen emotionalen Beziehungen zwischen Vorlesenden und Lernenden - auch zwischen den Lernenden untereinander - bestehen. Es fehlt an mitmenschlichem Attachment, so dass zu wenig Oxytozin produziert wird. Es fehlt an empathischer Resonanz und an Ungewissheiten im Rollenspiel, die Hoffnungen auf Belohnung erzeugen könnten. 
In neurobiologischer Sicht wird bestätigt, was schon lange zum pädagogischen Gemeingut gehört: Lernen muss Spaß machen. Nur Ausnahme-Personen, die außerordentliche charismatisch-didaktische Fähigkeiten mitbringen, können jene emotionalen Schubkräfte erzeugen, von denen der Lernertrag abhängt.

Hier kommen die Ergebnisse hochschuldidaktischer Untersuchungen ins Spiel. Beispielsweise berichtet der Didaktiker Heinz O. Gralki von einer Erhebung, in der es darum ging, die individuelle didaktische Kompetenz derjenigen zu überprüfen, die das Lernen anleiten. Der erstellte Index zeigte eine Verteilung ,in allen Fachbereichen nach dem Gauss'schen Modell: wenige Dozenten sind sehr gut, wenige sehr schlecht, die Mehrheit liegt in der Mitte.“ (Zit. nach: Grühn u. a. 1992: 38) Unter diesen Voraussetzungen ist klar, dass es Instruktions-Vorlesungen sowohl allgemeiner als auch spezialistischer Art in der Regel an charismatisch-emotionaler Durchschlagskraft fehlt.

Demgegenüber gibt es eine Ausnahme-Konstellation erfolgreicher Instruktions-Vorlesungen, in der frontal vortragende Rednerinnen und Redner auf ein Publikum mit Vorwissen treffen. Hier realisiert sich, dass Informationen, die es ins Arbeitsgedächtnis schaffen, im Langzeitgedächtnis ankommen, wenn sie an dort schon vorhandene Inputs andocken können. Vor allem wissenschaftliche Symposien und Konferenzen liefern solche Rahmenbedingungen. Dabei ist die Chance, dass es zu ertragreichem Lernen kommt, besonders groß, wenn der Wissensvorsprung der Vortragenden gegenüber dem zuhörenden Publikum gering ist und dieses umgekehrt darauf wartet, bestehende Lücken des behandelten Wissensbereichs durch Informationen der Vorlesenden zu schließen. Dabei kommt es nicht in erster Linie auf emotionale, sondern auf intellektuelle Erregung an.

Man stelle sich beispielsweise einen Hörsaal vor, in dem eine Mehrzahl ausgebildeter Philosophen und speziell am Thema „Religion und Gesellschaft" interessierter Fachleute einem Vortrag des akustisch nicht leicht zu verstehenden Jürgen Habermas folgen. Sie kennen das Problem. Sie überblicken die Antworten, die in der Geschichte der Philosophie bislang gegeben worden sind. Sie sind speziell mit den bisherigen Überlegungen des allseits anerkannten Denkers vertraut. Was wird er den bisherigen

und was wird er speziell seiner eigenen bisherigen Position hinzufügen? Alle wissen, dass der Redner selbst bemüht sein wird, die in ihn gesetzten Erwartungen an Erkenntnisgewinn zu erfüllen. Es ist diese außerordentliche Lern-Konstellation eines geringen Wissensgefälles, die plausibel macht, dass ertragreiches Lernen im Sinne des Erwerbs zusätzlichen - wenngleich möglicherweise minimalen Wissens - gelingt, ohne dass außerordentliches Charisma und starke Emotionalisierung im Spiel sein müssen.

Was die neurobiologische Bewertung von Instruktions-Vorlesungen mit Infotainment angeht, so kann beispielhaft auf Vorträge hingewiesen werden, die 
zu bestimmten Anlässen - Semestereröffnung, Emeritierung, Erinnerung an den Tod eines Wissenschaftlers, Künstlers oder Politikers - an Hochschulen gehalten werden. Dabei ist immer wieder auffällig, dass solche launig vorgetragenen Reden, die hier als Catch-all-Reden charakterisiert wurden, oft als enttäuschend empfunden werden. Vor allem fortgeschrittene Studierende kommentieren: Hat denn der berühmte Redner, der zu einer außerordentlichen Gelegenheit eingeladen wurde und den man endlich einmal live kennenlernen wollte, etwas Neues und noch nicht Bekanntes gesagt? Woran liegt die Enttäuschung?

Wenn es in der Regel nicht zu Standing Ovations kommt und die Reaktionen eher gedämpft ausfallen, so hängt das neurobiologisch gesehen damit zusammen, dass das neuronale Potential des Publikums, zumal das in den limbischen Abschnitten liegende Potential, nur in geringer Breite und Tiefe ausgeschöpft wird. Damit sich Lernende auf dem Niveau jenes eher verhaltenen Schmunzelns und zaghaften Lachens, von dem zum Beispiel gelungene Geburtstagsreden begleitet sind. In Verbindung mit dem Hauptgebot von Catchall-Reden (,Rede weniger, sage mehr!“) wird das neuronale Potential nicht im Sinne der Vermittlung umfassender Instruktionen ausgeschöpft. Es zeigt sich, dass das Bemühen, ein vielfältig zusammengesetztes Publikum (,catch-all“) in Stimmung zu bringen, dem Anspruch auf hohen Lernertrag nicht angemessen ist. Insoweit bestätigen sich die Zweifel an Vorlesungs-Shows, wie sie von Studierenden in ihrer Vorlesungs-Kritik angemeldet werden.

Es bleibt noch die zusammenfassende Bewertung von Vorlesungen, die dem Idealtyp der Impuls-Vorlesung nahekommen. Soweit auch sie auf die Vermittlung von Wissenselementen gerichtet sind, gelten dieselben Argumente, die aus neurobiologischer Sicht gegen Instruktions-Vorlesungen sprechen. In dem Maße jedoch, in dem Impuls-Vorlesungen über Wissensvermittlung hinauszielen, haben sie im Vorlesungsbetrieb der Hochschulen eine Sonderstellung, die verteidigt zu werden verdient. Sie wird von jener „episodischen Empirie“ (Frans de Waal) gestützt, die auf außerordentliche Eindrücke von Vorlesungen verweist, auf Erinnerungen an den Vortrag von Wissenschaftlern, die man „,in ihrem Denken, ihrem Ernst und ihrer Betroffenheit" (Jaspers) erlebt hat. Neurobiologisch erklärt sich diese Sonderstellung durch Ausnahme-Personen. Gestützt auf offensichtliche fachliche Kompetenz - verbunden mit je eigentümlich charismatischen Elementen sowohl rhetorischer als auch gesamtpersönlicher Ausstrahlung - sind sie in der Lage, ihr Publikum neuronal in ebenso umfassender wie differenzierter Weise herauszufordern, zu berühren und in Erregung zu versetzen. Das heißt aber auch, dass Impuls-Vorlesungen Seltenheitswert haben und nicht als selbstverständlicher Bestandteil des Vorlesungsbetriebs verstanden werden können. Es bedeutet im Besondern, dass sich nicht alle Lehrpersonen diesen Vorlesungstyp zutrauen können. 
Das gilt beispielsweise für schlecht vorbereitete und lustlos auftretende „Forscher-Gelehrte“, von denen die Studierenden im Hörsaal enttäuscht werden. Glaubwürdig wird etwa von dem Nobelpreisträger und späteren Präsidenten der Deutschen Forschungsgemeinschaft, Maier-Leibnitz, berichtet, er habe als Vorleser völlig versagt, sich an der Tafel immer wieder aufs Neue verheddert und auf diese Weise sich selbst und das Lerninstrument der ImpulsVorlesung blamiert. 


\section{Neurobiologisch informiertes dialogisches Lernen - traditionell und digital}

Wenn zunächst die schon im II. Kapitel vermittelten neurobiologischen Grundkenntnisse (II.1.) ergänzt und damit die Grundlage des in diesem Kapitel vorgestellten Konzepts dialogischen Lernens vervollständigt wird, so geht es um Folgendes: Beschrieben wird, was im neuronalen Lernapparat geschieht, wenn die über die Sinne in ihn eindringenden Impulse verarbeitet werden (Neuronal spurenarmes, vielspuriges und netzübergreifendes Lernen). Insbesondere im Anschluss an die Hinweise auf bewusstes und unbewusstes Lernen lässt sich skizzieren, wie Lernprozesse auf der synaptischen Ebene ablaufen. Darüber hinaus muss zum Ersten gefragt werden, was auf der synaptischen Ebene vor sich geht, wenn Lernprozesse - was in der Regel stillschweigend vorausgesetzt wird - durch Menschen vermittelt werden. Sie suchen den Kontakt zu Lernenden, um diese zu möglichst ertragreichem Lernen anzuleiten. An Beispielen sozialer Partnerschaften kann illustriert werden, worauf es dabei ankommt (Lernen im Kontakt mit Menschen). Zum Zweiten wird überlegt, was sich im Apparat der Lernenden auf der synaptischen Ebene tut, wenn sie mit Print-Texten, dem traditionell wichtigsten Lernmedium, in Kontakt treten (Lernen durch Lektüre von Print-Medien). Zum Dritten wird schließlich auf die synaptische Verarbeitung digitaler Materialien und speziell auf das digitale Multitasking eingegangen (V.1.).

Nach dieser Vorarbeit lässt sich ein Konzept dialogischen Lernens beschreiben, das auf seine Grundlagen zurückgeführt und aus analytischen Gründen zunächst ohne Bezug zu digitalen Lernmaterialien vorgestellt wird. Dem schließt sich eine modellhafte Anregung des Autors zum Interview-Format des Lernens an, das die im Zweier-Dialog liegenden Lernmöglichkeiten auszuschöpfen sucht (Nahkommunikation im Interview-Format) (V.2.).

Bei der Überprüfung des Potentials, das in digitalen Lernmaterialien steckt (V.3.), werden diese in einem ersten Schritt zunächst kursorisch vorgestellt. Wenn die unterschiedlichen digitalen Angebote - unter Einschluss des Einsatzes von Robotern - individuell und autonom genutzt werden, lassen sich tendenziell spurenarme Lerneffekte beobachten.

In einem zweiten Schritt kann diskutiert werden, inwieweit die digitalen Lernmaterialien geeignet sind, in das Konzept dialogischen Lernens eingefügt $\mathrm{zu}$ werden und in Kombination mit den traditionellen Lernformaten vielspuriges Lernen zu ermöglichen. Es geht um Präsenz-Lernen (anstelle von Präsenzlehre). Dabei gilt das besondere Augenmerk digitalisierten Vorlesungen, deren Dialog-Wert sich parallel zur traditionellen Vorlesungsversion einschätzen lässt (V.4). 
Wenn es zum dialogischen Lernens gehört, dass Lernprozesse in Nahkommunikation ablaufen, so muss insbesondere danach gefragt werden, was sich im Berufsalltag und in der Ausbildung derjenigen ändern müsste, die das Lernen anleiten (V.5.). Schließlich bleibt noch die Frage: Was würde sich für die Institution Hochschule und ihre Leistungsfähigkeit ändern, wenn sie in der Konkurrenz mit anderen Lern-Institutionen auf das Lernen im dialogischen Kleinformat umschalten würde (V.6.).

\section{Synaptische Grundlegung dialogischen Lernens}

\section{Neuronal spurenarmes, vielspuriges und netzübergreifendes Lernen}

Wenn Fachleute von der Plastizität des lernenden Gehirns sprechen, so bedienen sie sich des Begriffs der Bahnung neuronaler Netzwerke (priming). In Darstellungen, denen es um eine möglichst allgemein verständliche Erläuterung von neuronalen Lernprozessen $\mathrm{zu}$ tun ist, wird derselbe Vorgang mit einem Bild in Verbindung gebracht, nach dem die von außen kommenden Reize im Gehirn Spuren hinterlassen. Im Folgenden soll dieses Bild übernommen und dadurch ausdifferenziert werden, dass einerseits auf eher spurenarme sowie andererseits auf eher vielspurige und netzübergreifende Wirkungen Bezug genommen wird. Dabei ist zu berücksichtigen, dass die Spuren-Metapher den komplexen Vorgang der Bahnung nur unvollkommen trifft.

Das neuronale Netzwerk besteht aus einer riesigen Zahl von Neuronen und Gruppen von Neuronen. An ihren Kontaktstellen, den Synapsen, - - hier nicht im Einzelnen darzustellende - elektromagnetisch-chemische Austauschprozesse statt. Soweit sensorische Impulse die variabel miteinander verbundenen Neuronen und Neuronengruppen in einem segmentarischen Einzugsbereich treffen und zu gemeinsamem Feuern aktiviert werden, so sind die Effekte spurenarm. Kommt es zu neuronalen Aktivierungen, die das Netzwerk übergreifend erregen, so sind die Wirkungen vielspurig. Da es sich aber beim neuronalen Netzwerk um ein mehrdimensional aufgefächertes Gebilde handelt, muss bei der bloß horizontalen derLern-Spuren als spurenarm oder vielspurig immer ihre Mehrdimensionalität mitgedacht werden. Im Ganzen geht es um einen Aktivierungsvorgang, bei dem neue Neuronen hervorgebracht werden, die schon vorhandenen Neuronen wachsen und sich die neuronalen Verbindungen verstärken. Es sind diese auf synaptischer Stufe erfolgenden Veränderungen, die das ausmachen, was zu einem immer wieder revidierten Lernergebnis führt. 


\section{Lernen im Kontakt mit Menschen}

Nach den Vorstellungen des Schweizer Pädagogen Johann Heinrich Pestalozzi ist umfassende Bildung und Erziehung - nach einer vielfach zitierten Formulierung - davon abhängig, dass „Kopf, Herz und Hand“ einbezogen werden. Dazu bedarf es bei denjenigen, die das Lernen anleiten, einer Allround-Zuständigkeit, die in Nahbeziehungen wahrgenommen wird. Auf der Außenseite des Lernvorgangs zeigt sie sich in dem Bemühen, möglichst alle Sinne der Lernenden anzusprechen. In der für Pestalozzi noch nicht zugänglichen neurobiologischen Perspektive heißt das, dass der Lernapparat der Lernenden möglichst umfassend aktiviert werden muss. Am Beispiel von akademischen (Lern-)Karrieren von Studierenden ist schon gezeigt worden, wie sehr es im Verhältnis zu den Lehrpersonen auf Attachment als wichtiger Vorausssetzung für ertragreiches Lernen ankommt (II.2.).

Wie wirksam die Vermittlung des Lernens durch nahestehende Menschen sein kann, lässt sich zusätzlich an episodischen Hinweisen auf nicht-akademische (Lern-)Karrieren, beispielsweise von Fußballspielern, illustrieren. Diese sind insbesondere dann auf soziale Patenschaft angewiesen, wenn sie aus fernen Kulturen kommen. Hinzufügen lässt sich eine prägende mitmenschliche Erfahrung und Prägung, auf die beispielsweise der Schweizer „Nestbeschmutzer", Globalisierungskritiker und UN-Sonderberichterstatter für das Recht auf Nahrung, Jean Ziegler, sein ebenso bewegtes wie widersprüchliches Leben zurückführt.

Was die Karrieren ausländischer Fußballspieler angeht, so steht den vielen Beispielen ihres Scheiterns der Aufstieg zweier Brasilianer gegenüber. Bei beiden fällt auf, dass sie als Spieler verschiedener europäischer Clubs lange Durststrecken hatten, dann aber sehr erfolgreich waren. Beide spielten für den deutschen Top-Club FC Bayern München. Der eine, Giovane Elber, verband seine guten Leistungen damit, dass er sich von Bayern-Manager Uli Hoeneß „wie von einem Vater" behandelt fühlte (Internet-Eintrag). Der zweite, Costa Santos Dante, machte schon auf einer frühen Station seines schwierigen Wegs nach oben eine entsprechende Erfahrung. In einem autobiographischen Bericht schreibt er, dass er beim belgischen Verein Standard Lüttich auf einen Trainer gestoßen sei, der ihm Vertrauen geschenkt und ihm auf Portugiesisch geduldig erklärt habe, was für ihn unverständlich war. Dieser Trainer, so empfand Dante diese Zuwendung nicht anders als Elber, sei „eine Art Papa für ihn gewesen“ (Dante 2006: 128).

Was Jean Ziegler betrifft, so versteht er sich als „Linker, der für das Reich Gottes kämpft“, und führt diese Grundorientierung auf die Begegnung mit einem Jesuiten zurück, die er als 25-Jähriger hatte. Der Jesuit war in der Zeit der NS-Besatzung in Frankreich als Widerstandskämpfer verhaftet und nach 
Mauthausen deportiert worden. Über ihn sagt Ziegler: „Seine Haltung im Leben hat mich überzeugt. Mit ihm habe ich sehr viel diskutiert, er hat mir das Evangelium erklärt und die Dimension der Unsterblichkeit, der Auferstehung und der Ewigkeit deutlich gemacht. ... Ich bin konvertiert, und er hat mich getauft.“ (Interview in: Zeit-Magazin, 20.12.2017)

Das Gegenstück der hier herangezogenen Beispiele erfolgreicher Förderung von Karrieren besteht darin, dass Lernende auf Menschen treffen, denen es vor allem an der Fähigkeit mangelt, Attachment auszulösen und enge empathische Beziehungen aufzubauen (vgl. Kap. II.2.). Damit fehlen Voraussetzungen dafür, dass Lernprozesse neuronal vielspurig und netzübergreifend wirksam sind. Wären Fußballer wie Elber und Dante nur auf Trainer mit Schleiferformat gestoßen und wäre der junge Jean Ziegler nur zuwendungsunfähigen Menschen begegnet, so hätten vermutlich ihre (Lern-)Karrieren einen anderen und eher spurenarmen Verlauf genommen.

\section{Lernen beim Lesen von Print-Texten}

Von Lernvorgängen, die über Menschen vermittelt werden, unterscheidet sich das Lernen durch Lesen dadurch, dass Lernende, auch wenn sie möglicherweise von außen angeregt werden, sich den ihnen zugänglichen Lesestoff in Lernabsicht selbst auswählen. Sie machen sich an einen Print-Text heran, der sie inhaltlich interessiert. Ein zusätzlicher Unterschied zum menschenvermittelten Lernen besteht darin, dass es nur einen einzigen - visuellen - Zugang zur Innenseite des Lernprozesses gibt. Wie können sich dann aber Lernprozesse vielspurig und in netzübergreifender Breite entfalten?

Was hier zunächst zur Verarbeitung des Lesestoffs und zu den lokal für sie zuständigen neuronalen Strukturen skizziert werden kann, beruht in der Hauptsache auf Forschungen des französischen Neurobiologen Stanislas Dehaene und seiner Forschungsteams. In einer umfänglichen Studie Lesen (Dehaene 2010), die hier - ohne Einzelnachweise - ausgewertet wird, sind die wichtigsten Ergebnisse zusammengefasst, die wiederum auf die Forschung anderer Teams zurückgehen.

Von der Fovea, dem zentralen Bereich der Retina, wird eine beschränkte Zahl von Buchstaben in einem engen Zeitfenster aufgenommen. Welche Teile des neuronalen Netzwerks sind zuständig? Die zentrale Entdeckung von Dehaene u. a. besteht darin, dass der Lesestoff von einer spezialisierten Struktur der (Groß-)Hirnrinde aufgenommen wird. Diese sich in einer Übergangszone zwischen dem Hinterhauptlappen (Okzipitallappen) und dem Schläfenlappen (Temporallappen). Dabei handelt es sich um eine Region, die in beiden Gehirnhälften für Gesichtserkennung zuständig ist. In diesem Bereich hat sich indessen - eine Spezialisierung innerhalb der Spezialisierung - in der linken 
Hirnhälfte die Fähigkeit zur Verarbeitung von Lesestoff herausgebildet. Für Dehaene ist dies das Ergebnis eines langen Prozesses der Evolution, den er als Recycling versteht, ohne dass es dazu massiver Veränderungen der Gene bedurft hätte.

Neben der Eröffnung eines Zugangs zur Sprache gehört es zum Lesevorgang, dass - ebenso wie auf der synaptischen Ebene des Lernens - Lese-Spuren entstehen. Werden diese durch Leseübung konsolidiert, so kommt es zu Automatisierungen. Beim Lesen kann daher auf routinemäßige Leseerlebnisse zurückgegrif

Warum kommt es aber bei der Verarbeitung von Lesestoff zu vielspurig-mehrdimensionalen Wirkungen? Hier sind die Beobachtungen und Interpretationen der amerikanischen Autorin Maryanne Wolf hilfreich. Über die eher neuro-technischen Abläufe des Lesens hinausgehend, interessiert sie sich als Literaturwissenschaftlerin vor allem für das, was sich beim Lesen anspruchsvoller Texte herausbilden kann. Dabei nimmt sie neurobiologische Forschungsergebnisse auf, die von Dehaene u.a. vernachlässigt werden (v. a. Wolf 2009).

Sie entdeckt das Lesen als „Akt der verschlungenen Wege“ (Wolf 2009: 18), der - in ihrer Formulierung - zu ,deep reading“ führe. Das lesende Gehirn ist für sie „das stillschweigende Geschenk zum Nachdenken“ (Wolf 2009: 260). Es kann belegt werden, dass in dafür zuständigen neuronalen Arealen Buchstaben und Wörter aufgenommen werden. Diese lokale Erregung wird dann - wie sich plausibel machen lässt - an andere Teile des Netzwerks weitergeleitet. Dieser Zugriff tritt an die Stelle einer umfassenden Analyse des neuronalen Arbeitsraums, die wünschbar wäre, sich aber aufgrund seiner Komplexität (Wie hängt alles zusammen?) aus methodologischen Gründen kaum leisten lässt. Einsichtig ist, dass die netzübergreifende Ausstrahlung eines Lesestoffs davon abhängt, wie gedankenreich dieser ausgestaltet ist. Anhand der Lektüre eines Textes von Marcel Proust, auf dessen Einzelheiten hier nicht eingegangen werden kann, versucht die Autorin das Folgende zu zeigen:

Bei der Lektüre einzelner Text-Passagen werden Gedanken ausgelöst, die in den Einzugsbereich sowohl beider Hirnhälften als auch der vier Lappen der (Groß-)Hirnrinde fallen. Dabei muss das neuronale Netzwerk der Lernenden nicht notwendig über sämtliche Sinne aktiviert werden. Wer Wörter liest, hört beispielsweise auch Laute. Welche Teile des Netzwerks mobilisiert werden und wie der im Bereich etwa von Aufmerksamkeit, Wahrnehmung, und Motorik im Einzelnen verläuft, ist vom individuellen Lernstand der Lesenden abhängig. Dies gilt vor allem für die Einbeziehung der im Lernapparat je individuell aufgezeichneten Erinnerungen, die mit den Lese-Informationen verknüpft werden. Im Ganzen wird erkennbar, dass die Verarbeitung von Lese-Erlebnissen vielspurige und netzübergreifende Wirk- 
samkeit entfalten kann und daher gute Voraussetzungen für ertragreiches Lernen bietet.

Parallel zu fehlender Zuwendung beim menschenvermittelten Lernen sind die Voraussetzungen ungünstig, wenn es Leserinnen und Leser darauf anlegen, ihren Lesestoff möglichst schnell, überblicksartig und anstrengungslos zu bewältigen. Dieses von Texten hat zur Folge, dass die Lektüre zu spurenarmen Ergebnissen führt.

\section{Lernen beim Lesen von digitalen Texten und beim Multitasking}

Die Faszination, die von digitalen Medien ausgeht, gilt speziell auch für digitale Texte. Insbesondere zum Einsatz multifunktionaler Geräte (Smartphones und Laptops) zu Lernzwecken hinkt die Forschung jedoch hinterher. Im Besonderen ist unsicher, was im plastischen Gehirn geschähe, wenn eine „digital culture" das traditionelle Lesen ersetzen würde (Wolf 2009).

$\mathrm{Zu}$ vermuten ist, dass die auf dem beleuchteten Display verfügbaren Texte deshalb so attraktiv sind, weil sie sich durch Berühren und Wischen schnell bewegen lassen. Dies entspricht der ,neuronalen Ansprechbarkeit“ für Bewegungseindrücke, die schon vielfach beobachtet worden ist. ${ }^{57}$ Was mit einzelnen „digital shots" geschieht und wie sie auf synaptischer Stufe zusammengesetzt werden, müsste untersucht werden. Plausibel scheint, dass der Lernertrag digitaler Lektüre nicht weniger vom Gedankenreichtum der Texte und von vermittelnden zwischenmenschlichen Dialogen abhängt, als dies für andere Lernmaterialien gilt.

$\mathrm{Zu}$ der Frage, ob die Digitalisierung dazu beiträgt, die Voraussetzungen der Informationsverarbeitung und damit auch die Möglichkeiten ertragreichen Lernens maßgeblich zu verbessern, gibt es inzwischen sowohl empirische Forschung als auch, komplementär dazu, neurobiologische Grundlagenforschung. Die auch schon vor der Digitalisierung verbreitete Wunschvorstellung, möglichst viele Aufgaben gleichzeitig bewältigen zu können, kann auf der Basis

57 Die Firmen Bosch und Siemens Hausgeräte GmbH erteilten einer Arbeitsgruppe der Ulmer Universitätsklinik für Psychologie und Psychiatrie III (Leitung Markus Kiefer) den Auftrag, ihre Forschungsergebnisse zu einer für das Langzeitgedächtnis zentralen Teilstruktur des Gehirns in Empfehlungen zum innerbetrieblichen Unterricht umzusetzen. Die Arbeitsgruppe hob in ihren Vorschlägen in besonderer Weise hervor, dass die Ausbilderinnen und Ausbilder der Firma darauf achten müssten, den Lernenden im anschaulichen Unterricht möglichst viel Bewegungsangebote zu machen. (Vgl. den Internetauftritt der „Arbeitsgruppe Struktur des semantischen Gedächtnisses“ - unter Einschluss der Mitarbeiterliste und des Auftragsgebers, o. J.). Vgl. auch: Kiefer u. a. 2008: 47-58. 
der empirischen Studien nicht eingelöst werden. Der Einsatz multifunktionaler Geräte beim sogenannten Multitasking führt keineswegs zu Verbesserungen der Gedächtnisleistung. Im Gegenteil gibt es deutliche Anzeichen dafür, dass die Leistungsfähigkeit eher beeinträchtigt wird. ${ }^{58}$

In der neurobiologischen Grundlagenforschung sind, soweit sie auf das Multitasking bezogen werden kann, die aus der Bewusstseinsforschung kommenden Beobachtungen besonders aufschlussreich ${ }^{59}$ :

Bei der Beschreibung der Abläufe im Lernapparat der Lernenden, die dem Multitasking entgegenstehen, lässt sich davon ausgehen, dass bestimmte Neuronen und Neuronengruppen dieses Lernapparats besondere Zuständigkeiten haben. Sie betreffen beispielsweise die Verarbeitung von Buchstaben, Wörtern und Sätzen. Wie die Untersuchung der neuronalen Mechanismen zeigt, ist nun aber die erfolgreiche Bewältigung komplexer Aufgaben davon abhängig, dass die spezialisierten Neuronen und Neuronengruppen mit anderen und in unterschiedlicher Weise spezialisierten Neuronen und Neuronengruppen zusammenarbeiten. Die im genannten Beispiel für Buchstaben, Wörter und Sätze zuständigen Netzwerk-Abschnitte sind u.a. darauf angewiesen, dass andere Netz-Teile die Bearbeitung von Sprache mit Bedeutung versehen. Insoweit wird den Neuronen und Neuronengruppen des Lernapparats Multitasking abverlangt.

Der entscheidende Punkt ist, dass diese Multitasking-Aufgabe von den - im Beispiel für die Sprach- und Bedeutungs-Verarbeitung - zuständigen Neuronen und Neuronengruppen nur für eine der an den Lernapparat der Lernenden gerichteten Fragestellungen und nicht gleichzeitig für andere Fragestellungen geleistet werden kann. Die Bearbeitung von parallel an den Lernapparat gestellten Anforderungen muss daher so lange warten, bis gebundene Kapazität frei wird. Insoweit folgt aus der eingeschränkten Neuronen-Befähigung zum Multitasking, die für alle Teile des Lernapparats der Lernenden gilt, dass der Wunsch nach Multitasking nicht in Erfüllung gehen kann.

Was geschieht aber mit den Anforderungen im Wartestand, die nicht parallel bearbeitet werden können? Neuere Forschungsergebnisse verweisen darauf, dass auf dem Weg der Arbeitsteilung zwischen den beiden Gehirnhälften zwar ein zweiter Reiz aufgenommen werden kann. Kommen jedoch dritte und weitere Aufgaben hinzu, so entscheidet der Zufall darüber, ob und welche

58 Dies gilt auch dann, wenn die vorliegenden Daten weniger ,feindselig“ interpretiert werden, als dies Manfred Spitzer tut (Spitzer 2015: 61ff.); vgl. zu schon früheren Auseinandersetzungen mit dem Multitasking etwa: Pöppel 2008: 56ff.

59 Hier wird versucht, durch eigene Illustrationen gestützt, die wichtigsten Ergebnisse eines Forschungsberichts der Autoren Earl K. Miller und Timothy J. Buschmann (Brain Rhythms for Cognition and Consciousness, 2013) zusammenzufassen. 
der eingehenden Informationen im Langzeitgedächtnis aufgezeichnet werden (Korte 2017: 242-244). Insoweit zerplatzt der Traum vom Multitasking. An einem wichtigen Punkt zeigt sich, dass die an die Digitalisierung geknüpften Erwartungen relativiert werden müssen. Dies ist eine Erkenntnis, die sich bei der nachfolgenden Betrachtung des Einsatzes digitaler Lernmaterialien bestätigen wird (Kap. V.3.).

\section{Grundlagen eines Konzepts dialogischen Lernens}

\section{Warum dialogisches Lernen gebraucht wird}

Zu den Dispositionen, die im Gehirn angelegt sind und das menschliche Überleben ermöglichen, gehört die Lerndisposition. In ihr steckt das, was einführend „natürliche Neugier“ genannt wurde. Unter welchen Bedingungen wird sie entwickelt?

Für den Bereich der Hochschulen kann auf die Entwicklung mittelalterlicher Universitäten - z. B. auf das Bologna und Paris des 12. Jahrhunderts hingewiesen werden. Nichtadelige Studierende schlossen sich in dem Wunsch zusammen, aus ihrer gesellschaftlichen Unterprivilegierung herauszukommen (Seel 1985: 15-18). Die sie antreibende Lernbereitschaft und Neugier ergab sich aus einem Aufstiegsbedürfnis, das sich bei bildungsfernen Schichten auch in späteren Zeiten beobachten lässt. Bevor lernbegierige Arbeiterkinder an die Universitäten kamen, stillten vorausgehende Generationen ihren Wissensdurst auf zum Teil ungewöhnliche Weise. Beispielsweise organisierten Zigarrenarbeiter ihre stumpfsinnige Routinearbeit so, dass sie ihren Lohn mit einem Kollegen teilten, der dem Arbeitsteam aus gemeinsam interessierenden Texten vorlas (Buschak 1985: 40ff.).

Ohne Schwierigkeiten ist auch die überschäumende Neugierde nachvollziehbar, von der die aus dem Krieg zurückgekehrten Soldaten erzählen, als sie nach dem 2. Weltkrieg unter schwierigen materiellen und technischen Bedingungen an den behelfsmäßig wieder funktionstüchtig gemachten Universitäten studierten.

Belegen diese Beispiele, dass sich „natürliche Neugier“ bei besonders großen Herausforderungen entfalten kann, so heißt das im Umkehrschluss: Im Lernsystem muss es Anreize geben. Sowohl die allgemeine als auch die spezielle Lernsituation und die praktizierten Lernformen müssen so beschaffen sein, dass das im neuronalen Lernapparat liegende Potential ausgeschöpft wird. 


\section{Von obrigkeitlicher Lehre zum dialogischen Lernen}

Das hier umschriebene Konzept dialogischen Lernens steht didaktischen Vorstellungen nahe, die der australische Didaktiker John Hattie für den Schulbereich konzipiert hat. Sie wurden einführend zusammengefasst (Einführung 5.). Der Hauptunterschied ist, dass im Folgenden ein Konzept für Erwachsene entworfen wird - mit der Konsequenz, dass das bei Hattie stark betonte direktive Moment des Lernprozesses zurückgenommen und zugleich die Eigenarbeit der Lernenden hervorgehoben wird (vgl. Anm. 7).

Sowohl auf den Schul- als auch auf den Hochschulbereich sind zwei sich überschneidende didaktische Zugriffe bezogen, die seit dem Anfang der 2000er Jahre in den USA diskutiert werden. Es handelt sich um die Ansätze des Inverted Classroom und des Flipped Classroom (im Folg. nur: Inverted Classroom), aus deren Dunstkreis die nachfolgenden Anregungen stammen. $\mathrm{Zu}$ den deutschen Vertretern des Ansatzes gehören u. a. der Bielefelder Physiker und Mathematiker Jörn Loviscach sowie der Marburger Linguist und Hochschuldidaktiker Jürgen Handke. Indem es ihnen gelingt, das Lernen mit digitalen Medien in den Inverted-Classroom-Ansatz einzufügen, haben sie am hier entwickelten Konzept dialogischen Lernens Anteil (vgl. den 4. Abschnitt dieses Kapitels).

Das Konzept des Inverted Classroom wird im Anschluss an eine didaktische Position entwickelt, die auf die Formel eines ,Shift from teaching to learning" gebracht wurde. Es handelt sich dabei um eine Position, die schon in den 1990er Jahren - im Vorfeld der Inverted-Classroom-Diskussion - in den USA aufkam und im Umkreis der Deutschen Gesellschaft für Hochschuldidaktik (dghd) diskutiert wurde.

Der unmittelbare Anstoß zu den hier vorgestellten Vorschlägen kommt aus der lebensgeschichtlichen Verbindung des Autors mit der Studentenbewegung. Auf den Anteil, den sie und ihre Vorläufer daran hatten, dass über ,anders lernen“ an Hochschulen nachgedacht wurde, ist schon im I. Kapitel hingewiesen worden (I.2.). Sowohl der Widerstand gegen die inneruniversitären Entscheidungsstrukturen als auch die hier interessierenden Forderungen nach einer Revision der Lernprozesse waren in hohem Maße politisch motiviert. Das zeigt beispielsweise eine Publikation, in der Hochschulen - nach dem Negativ-Modell von Unternehmens-Strukturen - als „Lernfabriken“ kritisiert werden. (Leibfried 1967). Diese Kritik ist Teil eines nicht nur „von links" vorangetriebenen Demokratie-Diskurses. Karl Löwenstein nannte das Adenauer-Regime in seiner Verfassungslehre schon 1957 „demoautoritär“ (Löwenstein 1959: 93). Wie stark dieser Diskurs in den 1960er Jahren an Fahrt gewann und wie ungebrochen er dann in den 1970er Jahren fortgesetzt wurde, belegt die viel zitierte und weitverbreitete Aufforderung in Willy Brandts 1969er 
Regierungserklärung, „Demokratie (zu) wagen“. Diese Formel verdichtete die den Diskurs beherrschende Vorstellung, nach der sich alle Teilbereiche von Staat und Gesellschaft dem Demokratie-Gebot unterwerfen sollten. Es galt, wie es ein Buchtitel signalisierte, „Strategien der Demokratisierung“ zu entwickeln (Vilmar 1973).

In der Beförderung dieses Diskurses und gleichzeitiger Abhängigkeit von ihm trug die studentische Protestbewegung den Demokratie-Gedanken in die Hochschulen hinein. Es wurde gefordert, endlich über die Ausgestaltung von Lernprozessen nachzudenken. Das war der Kern vielfältiger Ausarbeitungen und Stellungnahmen der Bundesassistentenkonferenz (BAK) und später des ihr nachfolgenden Arbeitskreises für Hochschuldidaktik (AHD), die Hochschuldidaktik einforderten (Bartz 2007: 108). Bei den sehr allgemein formulierten Erwartungen, die an die Hochschulen gerichtet wurden, ging es um die Abschaffung der sogenannten Ordinarienuniversität. Mit dieser anti-obrigkeitlichen Formel verband sich ein doppelter Demokratie-Bezug. Im Negativen bestand er in heftiger Vorlesungskritik, die im IV. Kapitel schon belegt wurde (Kap. IV.2.). Komplementär dazu bestand der positive Demokratie-Bezug in der Forderung nach Partizipation, die dem Demokratie-Diskurs entnommen und in den Lernprozess eingeführt wurde: Lernende sollten ihn mitgestalten können, wie es schon die SDS-Hochschuldenkschrift von 1961 gefordert hatte (Kap. I.2.).

Die Hochschulgesetzgebung der 1970er Jahre zeigt, dass die aus der Studentenbewegung kommenden Forderungen, die inneruniversitären Entscheidungsstrukturen zu verändern, allesamt scheiterten (Reimann 1978). Das galt parallel auch für alle Bemühungen, die inneruniversitären Lernprozesse zu korrigieren. Auf das starre Festhalten am Vorlesungsprinzip ist oben hingewiesen worden (Kap.I.2.). Hochschuldidaktisch ambitionierte Initiativen wurden erst wieder in der zweiten Hälfte der 1990er Jahre ergriffen.

Im Umkreis der Deutschen Gesellschaft für Hochschuldidaktik (dghd), die 1971 an die Stelle des studentischen Arbeitskreises für Hochschuldidaktik (AHD) getreten war, wurden Forderungen aus der amerikanischen Didaktik-Diskussion aufgegriffen. Es ging dabei um eine Zielrichtung, die als neu deklariert und als „Shift from teaching to learning“ gefasst wurde (vgl. Barr/Tagg 1995 zur amerikanische Diskussion; Welbers u. a.: 2005 sowie Schneider/Wildt 2009 zur deutschen Diskussion). Schaut man sich das Ganze genauer an, so war der Ansatz jedoch gar nicht so neu.

Beim „learning“ geht es um nichts anderes als den Versuch, die Lernenden so weit wie irgend möglich dazu zu bringen, den Lernprozess in die eigenen Hände zu nehmen. Zur Illustration eignet sich der Hinweis auf das vielfach praktizierte sogenannte „Fishbowl“-Verfahren. Es diente es ursprünglich der Lösung von lässt sich jedoch problemlos auf Lernvorgän- 
ge übertragen. $\mathrm{Zu}$ dem anstehenden (Lern-)Thema gibt es Erst-Diskussionen ausgewählter Gruppenmitglieder. Sie bilden einen inneren Kreis (Fishbowl), der dadurch aufgebrochen und nach und nach aufgelöst wird, dass Mitglieder aus äußeren Kreisen die Plätze des inneren Kreises einnehmen. Auf diese Weise kommt es zu vielfältigen Bewegungen von außen nach innen und von innen nach außen. So kann das vorgegebene (Lern-)Problem möglichst von allen diskutiert werden, was sowohl zum individuellen als auch zum kollektiven (Lern-)Erfolg führt. Im Gegensatz dazu verbindet sich mit dem „teaching“, das aufgegeben und durch ,learning“ ersetzt werden soll, das Folgende: Der Lernstoff wird den Lernenden auf dem traditionellen Instruktionsweg mitgeteilt. Sie konsumieren ihn, ohne ihn im Dialog mit anderen zu bearbeiten und dabei für sich zu erschließen. Zur angestrebten Veränderung der Lernsituation, dem „Shift", gehört im Besonderen, dass die Lehrpersonen aus ihrer bisherigen Rolle heraustreten. Sie diktieren nicht mehr, sondern schlüpfen in eine dem Dialog dienende „Helfer-Rolle“ (Böss-Ostendorf/Senft 2018). ${ }^{60}$

Vergleicht man das aus der Studentenbewegung kommende Lernkonzept mit dem „Shift from teaching to learning“-Konzept und seiner Einführung in die deutsche Diskussion, so gibt es Unterschiede in der Begründung. Die terminologische Abhängigkeit des studentischen Konzepts vom Demokratie-Diskurs darf jedoch nicht darüber hinwegtäuschen, dass die Lern-Demokratie, die für den Hochschulbereich gefordert wird, wenig mit demokratischen Abläufen im Staat zu tun hat. Auch ohne ausführliche demokratietheoretische Erörterung ist klar, dass es in beiden Bereichen Partizipations-Ziele und Partizipations-Möglichkeiten gibt, die meilenweit voneinander entfernt sind. Das aber heißt, dass sowohl das studentische Konzept als auch das ,Shift“-Konzept auf erfahrungsbasierter Intuition beruht. Dies ist auch schon für das schulbezogene Konzept von John Hattie behauptet worden, das keine Abhängigkeiten von den Rahmenbedingungen erkennen lässt, in denen es konzipiert wurde.

Was aber haben dann alle drei Konzepte - parallel zu ihrer Ablehnung obrigkeitlicher Lehre - gemeinsam?

Alle drei bedürfen der neurobiologischen Begründungshilfe, um die sich schon Gerhard Roth für den Bereich der Schule bemüht hat und die im folgenden Kapitel auch noch für das digital angereicherte Konzept dialogischen Lernens gegeben wird. Die alle anderen Argumente tragende Begründung für das Konzept dialogischen Lernens liegt darin, dass der neuronale Lernapparat den dialogischen Lernansatz einfordert: Neuronale Spurenvielfalt und netzüber-

60 Die Frage nach der Rolle, die den Lehrpersonen im Konzept dialogischen Lernens zukommt, ist schon von Hattie aufgeworfen worden (vgl. Anm. 7). Sie wird im V. Kapitel im 5. Abschnitt aufgenommen. 
greifende Mechanismen, eine Voraussetzung für hohen Lernertrag, werden nur erzeugt, wenn der Lernprozess dialogisch angelegt ist. Dialogisches Lernen ist durch die folgenden Hauptmerkmale gekennzeichnet:

Diejenigen, die das Lernen anleiten, treffen Lernende in überschaubaren Gruppen. Es wird für eine Lernumgebung gesorgt, die der Nahkommunikation und dem Aufbau eines Vertrauensverhältnisses entgegenkommt.

Diejenigen, die das Lernen anleiten, machen Vorgaben zum gemeinsamen Lernstoff und sorgen - in Zusammenarbeit mit den Lernenden - für geeignete, möglichst breit gestreute Lernmaterialien.

Auf dieser Grundlage begegnen sich alle Beteiligten auf gleicher Höhe. Jenseits des Konkurrenzprinzips fordern sie sich gegenseitig heraus. Es kommt zum Austausch von Informationen und ihrer Interpretation, von Fragen und Antworten.

Wer bislang anti-obrigkeitliches Lernen an Hochschulen nur aus dem Bauch heraus gefordert und praktiziert hat, kann sich auf die Hilfestellung der Neurobiologie berufen. ${ }^{61}$

\section{Nahkommunikation im Interview-Format}

Wenn es in neurobiologischer Sicht für ertragreiches Lernen auf Nahkommunikation ankommt, dann heißt das, dass die in der Interview-Konstellation lie-

61 Die hier beschriebenen prinzipiellen Merkmale dialogischen Lernens müssten differenziert und erweitert werden durch Elemente von Dialog-Begleitung:

ELEMENTE VON DIALOG-BEGLEITUNG (stichwortartig):

1) Warnung/Entschiedene Einschränkung von Ablenkungseffekten ist geboten; durch Kürze der Begleitung von Dialogen (bis zu 15 Min.); durch Begleitung am Anfang und Schluss von Dialogen; durch Begleitung in Dialog-Pausen.

2) Andeutung der (zu korrigierenden) Ausgangslage: Lernszenario (weitgehend) ohne „Augenschmaus“, ohne „Ohrenschmaus“, ohne Bewegung und ohne Berührung.

3) Andeutung exemplarischer Korrekturen:

Studierende bringen Musikinstrumente mit. (Gemeinsames Hören schafft gemeinsame Lern-Plattform; durch Improvisation lassen sich lernthematische Bezüge herstellen.)

Studierende nutzen ihr Stimm-Potential. (Warum nur Einförmigkeit des bloßen Sprechens?) Stattdessen Laut-Artikulation (z. B.: In einem Seminar zur „Wahlund Wahlkampf-Analyse“ werden mit verteilten Rollen Ausschnitte aus Günter Grass' Tagebuch einer Schnecke artikuliert.) Lernthemenbezogene Sound-Varianten (CDs, DVDs; ,Sound-Gewitter“) werden z. B. in einem Seminar zum „Comparative Government" herangezogen, um deutsche Diktatur zu charakterisieren.); Bilder (Malerei), Fotos, Skulpturen; Theater- und Filmszenen; literarische Text-Ausschnitte. 
gende Möglichkeit besonders großer Nähe ausgenutzt werden sollte. Warum nicht diese Konstellation des Zweier-Dialogs gezielt in Lernabläufe einplanen? Die Initiative dazu kann von den Lernenden selbst kommen. Genauso kann es sein, dass diejenigen, die das Lernen anleiten, den Nah-Kontakt suchen und dabei besonders diejenigen Lernenden im Auge haben, denen die Mitarbeit in der Gruppe aus unterschiedlichen Gründen schwerfällt.

Im Radio und im Fernsehen gibt es ein Sendeformat, in dem sich professionelle Interviewerinnen bzw. Interviewer mit Interview-Partnerinnen bzw. -Partnern zum Zweier-Dialog treffen. Die beiden Beteiligten sind über vielfache Sinnesmodalitäten miteinander verbunden (außer sehen v. a.: hören, riechen, vorsichtig berühren) - eine vielgestaltige Gesprächsbeziehung die im Ausdruck (Inter-)View auf das gegenseitige Sehen verkürzt wird. Die interviewenden Personen sind Lernende, die am lebenslang erworbenen Wissen der Interviewten teilhaben wollen.

Es geht in einem hier beispielhaft ausgewählten Interview mit dem Neurobiologen Ernst Pöppel (Ludwig-Maximilians-Universität München) darum, Einblick in seine wichtigsten neurobiologischen Forschungsergebnisse zu bekommen (Alpha-Forum (WDR), Gespräch mit Iska Schreglmann v. 29.4.2010). Die Gesprächsgrundlage der Beteiligten besteht in ihrem allgemeinen Einverständnis darüber, dass es verschiedene Gründe dafür gibt, sich in einem bestimmten Lerngebiet, beispielsweise im Bereich der Neurobiologie, Wissen anzueignen. Bevor sich Interviewende und Interviewte zum Lerngespräch treffen, haben sie sich über Lernmaterialien, die dem speziellen Gespräch zugrunde liegen, verständigt. Die Interviewerin hat sich im obigen Beispiel in Texte von Ernst Pöppel eingelesen. Dieser hat sich auf die zu erwartenden Fragen vorbereitet.

Unter solchen Voraussetzungen kann der Dialog sowohl von den Interviewenden als auch von den Interviewten vorangetrieben werden. Eine gewisse Lenkungsfunktion kommt den Interviewten zu. Sie liegt einmal darin, dass die Lernmaterialien stark auf sie abgestimmt sind. Zum anderen sorgen die Interviewten als Fachleute für den Lernstoff dafür, dass die Gespräche nicht an Problemkomplexen vorbeigehen, die sie für wichtig halten. Ernst Pöppel erlaubt sich etwa, zur Klärung eines in seinen Augen wichtigen Sachverhalts etwas Zeit einzufordern (Interviewerin: „Gerne. Nur zu“). Umgekehrt kann aber die Gesprächsinitiative auch von den lernbegierigen Interviewenden ausgehen: Pöppel wird z. B. darum gebeten, zum Konzept eines bestimmten Buches längere Ausführungen zu machen.

Im Fortgang eines solchen Gesprächs führen gegenseitige Anregungen auch dazu, dass von beiden Seiten nicht erwartete Lernfelder zumindest gestreift werden. Was den Lernertrag dieses Sendeformats angeht, so kann es soweit die gemeinsame Gesprächsgrundlage stimmt - im günstigsten Fall zu 
einem Punkte kommen, an dem die Interviewten zu Lernenden werden. Nicht ohne Koketterie sagt Pöppel zur Interviewerin: „So (gemeint: wie Sie es in einer Vorwegnahme meiner Antwort gesagt haben) ist es, Sie sagen das viel besser."

Übertragen auf den Lernbetrieb an Hochschulen, der hier interessiert, ist in groben Zügen das folgende interviewartige Lernformat denkbar: Auf der einen Seite eines aktiven Interview-Duos steht ein einzelner lernbegieriger Mensch, der an die Stelle vieler Lernbegieriger tritt und sicher sein kann, dass er vom interviewten Gegenüber Die Interviewten - im Beispiel der Neurobiologe Ernst Pöppel - wissen zwar, dass es um Auskünfte zu dem von ihnen beherrschten Lernstoff geht. Ihnen ist aber klar, dass sie auch fachlich tieren können. Im Bereich der Neurobiologie können beispielsweise realitätsferne Versuchsanordnungen im Labor der Neuro-Ökonomie kritisiert werden (Wie kommen ökonomische Entscheidungen zustande?), die für routinierte Praktiker und Praktikerinnen der Forschung als etabliert gelten. Auch kommen von den Interviewenden Initiativen, die längere Informationsphasen initiieren können. Wenn von ihnen (mehr) Informationen eingefordert werden, so fehlt das Merkmal des problematischen octroi, der zum Frontalismus des Lernens gehört.

Am Ende einzelner Gesprächsphasen kann eine schriftliche Bilanz stehen. An diese lassen sich selbst wiederum neue Runden des Lerndialogs anschließen, der dann auch für das Lernen der Gesamtgruppe geöffnet werden kann.

\section{Zum Einsatz digitaler Lernmaterialien}

Auf die rudimentäre Forschung zum digitalen Angebot von Lesestoff ist schon aufmerksam gemacht worden. Sie betrifft auch den Einsatz anderer digitaler Lernmaterialien, so dass hier nur Beobachtungen und Überlegungen möglich sind. Vermieden werden sollte ein Weltanschauungskampf zwischen anciens und modernes, wie es ihn als Folge technologischer Entwicklungen in unterschiedlichen historischen Konstellationen immer wieder gegeben hat.

Auf der einen Seite lässt sich eine Neigung erkennen, in fast euphorischer, unkontrollierter Fortschritts- und Technik-Gläubigkeit eine Bildungsrevolution auszurufen. Dabei kommen auch ökonomische Interessen ins Spiel. Die Autoren einer aus der Bertelsmann-Stiftung kommende Publikation (Dräger/ Müller-Eiselt 2014) lassen sich durch Poocs (Personal open online courses) und Moocs (Massive open online courses) begeistern und halten es für möglich, dass die Massenschule und die Massen-Uni durch eine digitalisierte Lern- 
situation abgelöst werden. Obwohl sie einräumen, dass Lernen ein ,,sozialer Vorgang" sei, wird diese neue Situation folgendermaßen umschrieben: Die Individuen nutzen autonom digitale Lernangebote, die wie „maßgeschneidert“ zu ihren persönlichen Bedürfnissen passen. Das digital ermöglichte Revolutionäre besteht für Dräger/Müller-Eiselt in einer als Demokratisierung verstandenen Ausweitung des Bildungszugangs. Im Netz wird Wissen aufgezeichnet, das heruntergeladen und individuell verarbeitet werden kann (Dräger/MüllerEiselt 2014: bes. 51f.).

Dem steht eine Position entgegen, die beispielsweise von dem Schuldidaktiker Klaus Zierer vertreten wird. Gestützt auf den schon beschriebenen Ansatz von John Hattie und dessen Meta-Analyse (Einführung 5.), warnt der Autor vor technokratischer Behandlung von Bildungsproblemen. Seine Forderung, Lernen als Problem menschlicher Vermittlung zu betrachten, steht in Übereinstimmung mit dem hier - im Blick auf Hochschulen - entwickelten Konzept dialogischen Lernens (bes. Kap. V.). Indem Zierer jedoch sehr pauschal die Parole „Pädagogik vor Technik“ ausgibt (Zierer 2017: 100), verkennt er einerseits, dass es gute Möglichkeiten individuellen digitalisierten Lernens gibt, die nicht zwingend menschlicher Vermittlung bedürfen. Andererseits konzentriert sich Zierer fast ausschließlich auf den Schulbereich und ignoriert die insbesondere für den Hochschulbereich wichtige Frage, inwieweit sich digitale Lernmaterialien in menschenvermitteltes Lernen einfügen lassen.

\section{Digitales ,Entertainment“}

Unabhängig vom möglichen Lerngewinn haben die technischen Geräte (Smartphone, Tablet, Laptop), die den Zugang zur digitalen Welt bieten, etwas Faszinierendes und zugleich merkwürdig Amüsierendes. Der Reiz des Dings besteht darin, dass es für die meisten Benutzerinnen und Benutzer völlig undurchsichtig und unergründlich ist und ihnen dennoch durch Tastendruck oder einfaches Wischen beherrschbar erscheint. Warum sollte man mit dem Ding nicht auch lustvoll lernen können? In der schon genannten Publikation von Dräger/Müller-Eiselt wird ein alltägliches Lernszenario beschrieben, in dem es um die digitale Anleitung zum Lesen geht (Dräger/Müller-Eiselt 2014: 76.):

Da beklagt sich ein Vater, dass sein Sohn nur an Hörbüchern interessiert war und „keine Lust hatte“, Bücher in die Hand zu nehmen. Im zweiten Schuljahr habe der Lehrer des Jungen seinen Schülerinnen und Schülern eine Internet-Plattform angeboten, auf der sie zu jedem gelesenen Buch Fragen stellen konnten, schnell Antworten erhielten, je nach Leistung Punkte bekamen und mit Urkunden ausgezeichnet wurden. Ohne diesen digital-spielerischen Ansatz, so der Vater, würde sein Sohn ,wahrscheinlich noch immer auf seinem Sitzsack hocken und Hörbücher hören“. 
Das Beispiel illustriert, wie durchschlagskräftig Anreize zu fröhlichem Lernen sein können. ${ }^{6}$ Auch beim Lernen von Erwachsenen geht es um die Wirksamkeit des Faktors Abwechslung. Nach der sorgfältigen Auswertung vieler Einzeluntersuchungen durch die amerikanischen Lernexperten Richard A. Schmidt und Robert Bjork wird dieser Faktor zwar nicht das mechanische Üben und anstrengende Wiederholen - beispielsweise beim Lesen - machen. Es kann aber, geschickt eingesetzt, dazu beitragen, den Übungserfolg auf lange Sicht zu verbessern. Dies gilt für die Einübung in motorischen, verbalen, wissenschaftlichen und sportlichen Bereichen (nach Carey 2015: 224, 232f.). Übungspraktisch lässt sich der Abwechslungseffekt beispielsweise dadurch erreichen, dass der Lernstoff portioniert wird (Carey 2015: 108ff.).

Abwechslung lässt sich nicht von Ablenkung und Zerstreuung trennen. Zusammengenommen machen sie das aus, was sich in Pädagogik und Didaktik als spielerisches Lernen, ein unbestrittenes Förder-Prinzip, etabliert hat und durch die Neurobiologie gestützt wird (Kap.II.2., Kap. IV.3.). Damit ist keineswegs gemeint, dass die Nutzung digitaler Medien nicht dazu führen kann, dass Lernprozesse sowohl bei Jugendlichen als auch bei Erwachsenen in höchst schädlicher Weise gestört und verhindert werden. Auch sind gefährliche Suchteffekte unübersehbar (Montag 2018). Die alleinige Konzentration auf Probleme der Spielsucht, die beispielsweise in Manfred Spitzers Publikation zur „Cyberkrankheit“ auffällt (Spitzer 2017: 85ff.), übersieht die vielfältigen Lernhilfen, die im digitalen Spiel-Angebot liegen. Wenn man die für Lernprozesse zuständigen neuronalen Strukturen in analogisierender Weise mit Muskelgewebe vergleicht, so brauchen auch sie Entspannung und Erholung. Die Möglichkeiten, die sorgfältig und in Spielphasen einsetzbare Materialien bieten, sollten daher genauestens überprüft werden.

\section{Ein Roboter im Hörsaal}

Ein Beispiel für die „Entertainment“-Effekte der digitalen Technologie lieferte der Linguist und Hochschuldidaktiker Jürgen Hanke im Herbst 2017. Er löste ein hier nicht zu belegendes Medien-Echo aus, als er einen niedlich ausschau-

62 Problematisch ist indessen Drägers und Müller-Eiselts globale Botschaft: Erst seit wir über digitale Anreize verfügen, bringen wir Einzelne zum Lesen. Da wir aber gut daran tun, die Anstrengung des Lesens auch anderen Schülern zu ersparen, sollten wir möglichst alle auf den Weg der ,digital unterstützten Leselust“ bringen. Wo bleibt dann aber das Bemühen der Schülerinnen und Schüler darum, jene umfassenden Möglichkeiten des Lesens eigenständig auszuschöpfen, die in Kap. V.2. betrachtet wurden?

Der Roboter ist die „Zentralfigur“ eines vom Bundesforschungsministerium geförderten Projekts (Humanoid Emotional Assistant Robots in Technology/H.E.A.R.T.). 
enden Roboter in den Hörsaal mitbrachte. Wie reagieren die Studierenden über das Spaßmoment hinaus - und was halten insbesondere die Kollegen von Handkes schon jahrelangen Bemühungen, für den Einsatz digitaler Lernmaterialien in der Hochschul-Lehre zu werben?

Was den Einsatz von Robotern angeht, so stellt sich die prinzipielle Frage, ob der im Hörsaal als Assistent vorgestellte „Kollege“ auf längere Sicht dazu in der Lage sein wird, das Lernen selbständig anzuleiten, auf die Handke nur eine unzureichende Antwort hatte. Zur Begründung seines entschlossenen Neins wäre die ausführliche Auseinandersetzung mit einer kaum mehr zu überblickenden Literatur zur Künstlichen Intelligenz und ihrer Zukunft erforderlich. In aller Kürze kann hier auf die dort beschriebenen - ebenso ambitionierten wie großzügig - Projekte hingewiesen werden, in denen es darum geht, die natürlichen Neuronen des Gehirns und seiner Netzwerke durch künstliche Netzwerke nachzukonstruieren. Dabei wird das Folgende deutlich (u. a. Dormehl 2016):

Mit ihrer Softwarearchitektur werden zwar künstliche Systeme den natürlichen Systemen und den in ihnen ablaufenden Prozessen immer ähnlicher.

Pläne in der Richtung naturidentischer Nachbildungen lassen sich indessen nicht realisieren. Unter anderem ist die Ausdifferenzierung der künstlichen Systeme ebenso unbefriedigend wie das Material ihrer Chips. Die natürlichen Neurotransmitter können nicht angemessen ersetzt werden. Dem künstlichen Nachbau der elektronischen Netzverbindungen fehlt die naturidentische chemische Komponente (Urchs 2002: 211-218).

Nun lässt sich argumentieren, dass wir Menschen „eine bemerkenswert tolerante Einstellung haben“, wenn es darum geht, den Mitmenschen ,gelegentlich fehlerhaftes Verhalten" zuzugestehen. Müsste man nicht fairerweise darauf verzichten, die „Kriterien von nicht-menschlichen Systemen“ zu hoch anzusetzen (Urchs 2002: 227)? Gegenüber solchen Überlegungen muss auf unzweifelhaft vorhandene Reaktionen des Fremdelns verwiesen werden, die sich mit dem Spaß am Roboter als lustigem Kerl verbinden. Auch die im Forschungsbereich des „Affective Computing“ entwickelten Anpassungsfähigkeiten denkender Maschinen - beispielsweise die Fähigkeit der Gesichtserkennung - ändert nichts daran, dass sie die menschlichen Bedürfnisse nach Bindung nicht befriedigen können (II.2./V.2.). Selbst Menschen, die durch Alter oder Krankheit in ihren Wahrnehmungsfähigkeiten stark beeinträchtigt sind, haben noch differenzierte Bedürfnisse nach Zuwendung. Diese werden durch maschinenhaft verfremdete Lernpartnerinnen und Lernpartner nicht befriedigt.

Nun zielt aber Jürgen Handke mit seiner Werbung für die Digitalisierung an Hochschulen nicht in erster Linie auf den Einsatz von Robotern im Hörsaal. Im Angebot hat er - und haben auch andere Didaktiker - eine Reihe von längst erprobten, in vielen kompetenten Publikationen begründeten Vorschlä- 
gen zu digitalem Lernen (besonders: Handke 2015). Handke unterscheidet Macro-Lectures von Micro-Lectures (hier auch: Mikro-Texte). Bei Ersteren handelt es sich um zeitlich ausgedehnte Lehrvideos mit Vorlesungscharakter, in denen komplexe Inhalte (zumeist) durch Lehrpersonen vermittelt werden. Unter dem Gesichtspunkt ihres Lernertrags werden sie im Folgenden mit den traditionellen Vorlesungen verglichen.

Was die Micro-Lectures angeht, so werden ihnen im Folgenden alle VideoAufzeichnungen zugerechnet, die in beschränktem zeitlichen Rahmen (bis zu 20 Min.) inhaltlich reduzierte Angebote machen und zum großen Teil ohne personelle Komponenten produziert werden. Dazu gehören auch Instruktionen, die aus der Software von Robotern kommen. Ebenso erfasst sind E-Learning-Materialien, die schon seit vielen Jahren - auch in Audioform - in sehr unterschiedlichen Versionen genutzt werden. Je nach Version müssen sie den Makro- oder Mikro-Formaten zugerechnet werden.

Die knappste Form von digitalen Mikro-Texten, die hier nur am Rande erwähnt werden, sind Kurz- und Spezialinformationen, die der organisatorischen Entlastung von Lehrpersonen dienen. Auf solche Hilfestellung bezog sich Handke, als er bei seinem Auftritt mit dem Roboter sagte, er wolle nie mehr die Frage beantworten: „Wann ist der Termin der Abschluss-Klausur?“ Dazu braucht man freilich keinen Roboter, da Informationsbedürfnisse dieser organisatorisch-technischen Art leicht auf der Netz-Plattform des von Handke durchgeführten Kurses befriedigt werden können.

\section{Digitale Assistenz bei der Einübung von Automatismen und beim Lernen von Fremdsprachen}

eben den Instruktionen organisatorisch-technischer Art werden in die Software von Robotern auch inhaltliche Informationen einprogrammiert. Soweit diese außerhalb von Robotern in Studios mit unterschiedlicher Aufnahmetechnik aufgezeichnet werden, handelt es sich um Lernmaterialien des zeitlich und inhaltlich beschränkten Typs, also digitale Mikro-Texte. Aufbereitet werden Fakten beliebiger Themenkreise, wie sie sich auch in traditionellen Lehrbüchern Wenn dort komplizierte Sachverhalte abgehandelt werden, ist das oft eine sehr zähe Lektüre. Zur digitalen Aufmunterung sind daher MikroTexte gefragt, die sich beliebig oft abrufen lassen. Denen, die das Lernen anleiten, bleibt es dadurch erspart, auf wiederholt gestellte Fragen stets dieselben Antworten geben zu müssen.

Im Bereich der Sozialwissenschaften gilt das beispielsweise für die Vermittlung von Grundkenntnissen in methodologischen Fragen. Lernende begegnen Autorinnen und Autoren, die es einzuordnen gilt. Welchen theoretischen Richtungen gehören sie an? Die Lernenden selbst haben das Bedürfnis, 
sich über ihren eigenen methodologischen Weg Klarheit zu verschaffen. Die dies erschwerende Trägheit des menschlichen Bewusstseins, auf die hingewiesen wurde (Kap. II.1.), lässt sich am ehesten dadurch überlisten, dass im sogenannten prozeduralen Gedächtnis Automatismen angelegt werden. In Sachen Theorie-Verständnis bestehen sie darin, dass in der Auseinandersetzung mit fremden und eigenen Positionen immer wieder der Bezug zu den - digital wiederholt aufgerufenen - Richtungen hergestellt wird. Auf diese Weise kommt es automatisch zur fortlaufenden Überprüfung sowohl fremder als auch eigener theoretischer Orientierungen, um die gerungen wird. Man könnte auch sagen, dass es sich hier um ein theoretisches Positions-Training handelt, das mit Hilfe digitaler Medien automatisiert wird.

Dem entsprechen die Automatismen, die im Fußballtraining außerhalb des Fußballfelds eingeübt werden. Auch Fußball-Unkundigen wird es nicht schwerfallen, den Einsatz digitaler Lernmaterialien nachzuvollziehen: Da geht es beispielsweise darum, die Spielerinnen und Spieler dazu zu bringen, den Ball möglichst in den eigenen Reihen zu halten und ihn, wenn er verlorengeht, umgehend wieder zu erobern. In enger Verbindung mit diesem Ball-BesitzSpiel steht das Pass-Spiel, bei dem der Ball ebenso präzise wie schnell weitergeleitet wird. Die nach einem bestimmtem Spiel oder auch nach bestimmten Trainingseinheiten erstellte Video-Analyse (= digitaler Mikro-Text) gibt Aufschluss darüber, wie schnell der Ball nach seiner Annahme weitergegeben und ob der Mitspieler oder die Mitspielerin passgenau angespielt wurde; ob die Möglichkeit gesehen und genutzt wurde, rechts oder links außen eine Raum-Lücke aufzureißen; besser noch: den Ball hinter die Kette der gegnerischen Verteidigung zu schicken.

Um ähnliche, videogestützte Einstudierungen von Automatismen geht es etwa bei Versuchen, den Ball durch „Pressing“ zu gewinnen, um dann - noch bevor das gegnerische Team sich formieren kann - das Umschaltspiel anzukurbeln. Den Torjägern und -jägerinnen, speziell solchen, die in torreifen Szenen nicht zum Abschluss gekommen sind, werden solche Szenarios per Video beispielhaft vorgespielt, um zum Erfolg führende Abläufe einzustudieren. Diese gilt es dann auf dem Platz umzusetzen.

Der Vergleich zwischen dem Theorie-Lernen und dem Fußball-Lernen macht deutlich, wie sich digitale Mikro-Texte in unterschiedlichen Lernbereichen einsetzen lassen. Im einen Fall werden dem Lernapparat mit Hilfe von Videos neuronale Mechanismen vermittelt, die auf Abruf automatisierte Denk-Bewegungen auslösen. Im andern Fall kommt es dank Video-Analyse dazu, dass dem Lernapparat neuronale Mechanismen eingeprägt werden, die auf Abruf zu automatisierten Körperbewegungen führen. In beiden Fällen handelt es sich indessen nur um punktuelle spezieller Lernakte, nicht aber darum, Lernprozesse umfassend zu steuern. 
Eine ähnliche Konstellation, in der digitale Lernhilfen assistierend eingesetzt werden können, ist bei der Einübung von Fremdsprachen erkennbar, ein unverzichtbarer Bestandteil des Lernens an Hochschulen. Die meisten Fachleute sind sich darüber einig, dass es zur Einführung in das hochkomplexe Regelwerk von Sprachen umfassender Lernprozesse mit menschlicher Vermittlung bedarf. Jedoch belegt die Forschung (Macedonia 201763), dass künstliche Lehrpersonen beim Lernen von Wörtern hilfreich sein können. Die Software dieser Roboter ist so programmierbar, dass sie Wörter vorsprechen und zur Wiederholung auffordern können, wenn die Aussprache ihren Vorgaben nicht entspricht. Dabei erhöht es die Merkfähigkeit der Lernenden, wenn die künstlichen Lehrpersonen die Wörter, die sie vorsprechen, mit Gesten begleiten und die Schülerinnen und Schüler - während sie Wörter aufnehmen - dieselben Bewegungen ausführen

Lernfördernd wirkt auch, dass der Kontakt zu den künstlichen Lehrpersonen Spaß macht und zu diesem Entertainment-Effekt auch gehört, dass Belohnungserwartungen der Lernenden durch das Lob der künstlichen Lehrpersonen erfüllt werden.

\section{Die Lektüre digitaler Mikro-Texte im Vergleich mit traditioneller Print-Text-Lektüre}

Am Vergleich zwischen digital aufgezeichneten Texten mit Print-Texten besteht schon längere Zeit ein großes Forschungsinteresse. Zur Beantwortung der Frage, ob es Unterschiede im Lernertrag beider Text-Arten gibt, können die Ergebnisse einer neueren Studie des Tübinger Forschungsinstituts für angewandte kognitive Psychologie und Medienpsychologie herangezogen werden (Martin Merkt u. a. 2011). Dabei muss die Differenziertheit des methodischen Zugriffs und seiner Ergebnisse vereinfacht werden.

Der faktenorientierte 16-minütige Text zur Gründungsgeschichte der Bundesrepublik in den Jahren 1945-1950, zu dem Schülerinnen und Schüler der gymnasialen Oberstufe befragt wurden, lässt sich den Mikro-Texten zuordnen. Als Forschungsmaterial ersich auf der Stufe spurenarmen Lernens, da er nicht jene weitgehenden Assoziationen auslösen kann, die zur Spurenvielfalt führen (V.2.). Von Interesse ist gleichwohl, dass es auch bei eher bescheidenen Anforderungen an den Lernertrag Schattierungen gibt.

Den Probandinnen und Probanden wurde der Print-Text in einer durch Bildmaterial illustrierten Form, der digitale Text in zwei Versionen vorgelegt:

63 Hier werden neueste Forschungsergebnisse referiert, die von der Psycho-Linguistin Manuela Macedonia zusammengefasst werden (mit Literaturangaben). 
Das eine Video erlaubte es den Probanden, bei der Erschließung der Fakten des Textes eine Stop-and-Go-Funktion sowie eine Funktion des Forward-and-Rewind zu benutzen (common video). In der zweiten Version bestand zusätzlich die Möglichkeit, den Text mit Hilfe einer Zeitleiste, eines Inhaltsverzeichnisses und eines Registers differenzierter zu erschließen (enhanced video).

Die Studie bezieht sich auf die Ergebnisse vorausgehender Forschung. Hier hatte sich erwiesen, dass der Ertrag der Print-Lektüre der Video-Lektüre überlegen war, sofern Letztere ohne Steuerungsmöglichkeiten praktiziert wurde. Demgegenüber führte die Tübinger Studie im Fall des common video zum gegenteiligen Ergebnis: Der Fakten-Ertrag der Print-Lektüre wurde von dem der digitalen Lektüre-Version übertroffen.

Als Schlussfolgerung ergibt sich daraus, dass auf der spurenarmen LernStufe neben das traditionelle Lernangebot der Print-Lektüre ein digitales wenngleich steuerungsabhängiges - Angebot getreten ist, dessen Ertrag zumindest als gleichwertig, wenn nicht als höherwertig eingeschätzt werden muss.

Zudem ist die sich aus den Tübinger Ergebnissen abzuleitende Einsicht beachtenswert, dass das enhanced video - entgegen den Erwartungen der Forschenden - keinen Mehrertrag liefert. Das Angebot, zusätzliche Möglichkeiten der Steuerung (Zeitleiste, Inhaltsverzeichnis, Register) zu nutzen, um auf diese Weise einen digitalen Text differenzierter und sorgfältiger zu durchdringen, wurde von den Probandinnen und Probanden nicht angenommen.

\section{Lernen mit digitalen Macro-Lectures (digitalisierte Vorlesungen/Moocs)}

Digitalisierte Vorlesungen setzen die Vorlesungstradition fort und wecken vielleicht deshalb besonders hohe Erwartungen. Es war der Harvard-Informatiker Sebastian Thrun, der als Erster mit einer online gestellten Macro-Lecture (Einführung in die künstliche Intelligenz, 2011) großen Zuspruch erhielt. Danach kam es nach diesem Vorbild zu einem Hype von Online-Angeboten, in denen prominente Professoren reputierter Universitäten aufregenden Lernstoff in Form von digitalisierten Vorlesungen präsentierten. Auch nach ihrer zumindest teilweisen Kommerzialisierung sind diese vor allem deshalb hoch attraktiv, weil es sich um Massive Open Online Courses (Moocs) handelt, die allen lernbegierigen Menschen Möglichkeiten ortsunabhängigen Lernens versprechen (Dräger/Müller-Eiselt 2014: 51ff.).

Diese hohen Erwartungen können aber die Moocs und auch solche MacroLectures, die außerhalb des Hörsaals produziert werden, nicht erfüllen. Die Argumente, die im IV. Kapitel (IV.3.) in neurobiologischer Perspektive gegen die traditionellen Massenvorlesungen formuliert wurden, gelten ebenso für digitale Macro-Lectures. Wenn Studierende eine Lecture herunterladen, sie autonom und isoliert am Computer sitzend aufnehmen, so sind sie in ihrem 
Aufnahmevermögen nicht weniger begrenzt als die im Hörsaal Lernenden. Noch wichtiger ist, dass es auch im digitalen Lernformat nur zu beschränkter Mobilisierung emotionaler Schubkräfte kommt.

Das ahnen die und -Praktiker durchaus. So kamen bei Sebastian Thrun unter dem Eindruck hoher Abbrecherquoten und sehr unbefriedigender Prüfungsabschlüsse Zweifel auf, ob die Moocs tatsächlich für einen neuen, universal einsetzbaren und erfolgreichen Lernzugriff stehen. Bei dem Versuch, die Mängel des „lousy product“ (Thrun) zu erkennen und Abhilfe zu schaffen, verweist Thrun in einem Interview darauf, dass es den am Bildschirm Lernenden an Betreuung fehle („Moocs sind bisher nicht gut genug“, in: Tagesspiegel, 15.01.2014). Sein Udacity-Unternehmen habe daher eine 24-Stunden-Hotline eingerichtet, die es erlaube, fachliche Fragen zu stellen. Hinzu komme ein Service, bei dem die Lernenden mit Tutorinnen und Tutoren reden können.

Diese Mooc-Analyse und die aus ihr abgeleiteten Konsequenzen verweisen auf dieselben Probleme, die das Lernen im Hörsaal aufwirft. Um sie zu bearbeiten, werden die Hörenden vielerorts dazu aufgefordert, teilweise schon während des Vortrags - zumindest aber danach - Fragen zu stellen. Tutorinnen und Tutoren sollen bei der Vor- und Nachbereitung des Vorlesungsstoffs helfen.

Im nächsten Abschnitt (V.4.) wird es um die Möglichkeiten gehen, traditionelle mit digitalen Lernmaterialien zu verbinden. Es muss indessen klar sein, dass die bei Moocs im Video auftretenden Vorlesenden körperlich und damit auch mimisch und gestisch für die Lernenden nicht wirklich da sind. Ihre Präsenz ist digital verfremdet. Abgeschwächt wird dieser Mangel dadurch, dass die Vorlesenden zumindest mit ihrer Stimme präsent sind und dadurch - wie beim Radiohören - Lernen durch konzentriertes Hören ermöglicht wird. Bekräftigt wird jedoch die digitale Verfremdung der Vorlesenden dadurch, dass es - im Unterschied zum Hörsaal - keinen Blickkontakt und damit keinen direkten mimischen und gestischen Bezug zu ihnen gibt. Die Vorlesenden wenden sich an ein Publikum, das die autonom und isoliert am Computer Lernenden nicht kennen.

Hinzu kommt, dass es den am Computer Lernenden an jeglicher situativer Abwechslung mangelt. Demgegenüber sind die im Hörsaal Lernenden zum einen dadurch miteinander verbunden, dass sie gemeinsam sehen, hören und Beifall spenden. Zum andern sind sie zumindest stillschweigend dabei, wenn die Mit-Lernenden in unterschiedlicher Weise auf das reagieren, was die Vorlesenden ihnen bieten. Dies sind Faktoren neuronaler Erregung, so schwach sie im Hörsaal auch sein mögen, die den isoliert am Computer Lernenden fehlen.

Zudem wurden auch die

Erwartungen, die Moocs könnten zur digitalen Ausgangsbasis massiver Kooperation im Netz führen (Dräger/ 
Müller-Eiselt 2015: 88ff.), nicht erfüllt. Auf dieses weitgehende Ausbleiben der Bildung von Online-Communities weist nicht nur Thrun hin. Es entspricht auch den Erfahrungen des im folgenden Abschnitt noch ausführlicher vorzustellenden Bielefelder Hochschullehrers Jörn Loviscach. Ihn irritieren nicht nur die hohen Moocs-Abbruchquoten. In Übereinstimmung mit Kolleginnen und Kollegen registriert er auch, dass das Angebot an Studierende, sich in Mooc-Foren und auf Lernplattformen einzubringen, nur von sehr wenigen genutzt wird (Vortrag auf ,futur iii“ 2016).

Im Ganzen werden in neurobiologischer Perspektive die Hauptgründe dafür erkennbar, dass der alleinige Einsatz von Macro-Lectures zwar den Anspruch auf Innovation einlöst, zugleich aber die Anforderungen ertragreichen Lernens in Nahkommunikation nicht erfüllen kann.

\section{Digitales Präsenz-Lernen im Rahmen des Konzepts dialogischen Lernens}

Der Abschlussbericht eines vom Stifterverband für die deutsche Wissenschaft eingesetzten Expertengremiums enthält Handlungsempfehlungen zur „Hochschulbildung in Zeiten der Digitalisierung“. Sie mahnen die „Entwicklung methodisch-didaktisch wirksamer digitaler Lehrkonzepte und -formate" in Verbindung mit „entsprechender Erweiterung von Betreuungskonzepten“ an (Hochschulforum Digitalisierung 2016). Was bedeutet aber eine solche sehr allgemein formulierte Forderung, wie sie auch in anderen didaktisch interessierten Veröffentlichungen erhoben wird? In neurobiologischer Perspektive gibt es dazu zwei miteinander verknüpfte Antworten. Die eine wurde bei der Vorstellung der wichtigsten digitalen Lernmaterialien schon gegeben: Diese müssen in ihren zeitlichen und inhaltlichen Anforderungen so gestaltet sein, dass sie der Aufnahmekapazität und den Mechanismen des neuronalen Lernapparats der Studierenden angemessen sind. Die Charakterisierung dieser digitalen Materialien ergab, dass ihre individuelle und autonome Verarbeitung zu tendenziell eher spurenarmem Lernertrag führt. Daher besteht die Chance vielspuriger und netzübergreifender Lerneffekte nur, wenn die Materialien in eine dialogische Konstellation eingebracht werden. Die zweite Antwort lautet entsprechend: Die Materialien müssen sich auch für einen Disput und dazu eignen, einen lebhaften Austausch von Informationen und Interpretationen auszulösen. Dies ist die Voraussetzung für starke neuronale Erregung und hohen Lernertrag.

Jenseits solcher Einschätzungen sich das schon erwähnte, „bildungsrevolutionäre" Plädoyer, das von der Bertelsmann-Stiftung verbreitet wird (Dräger/Müller-Eiselt 2015). In dieselbe Kerbe schlägt auch der Leiter des Hasso-Plattner-Instituts (Potsdam), Christoph Meinel. Er setzt sich für eine 
radikale Umschaltung des Bildungssystems auf digitale Medien ein und nutzt dafür die Plattform „Digitalisierung in Bildung und Wissenschaft“, die 2015 vom Bundesministerium für Forschung und Wissenschaft eingerichtet wurde. In einem weit gestreuten Vortrag entwirft Meinel eine „Vision für die digitale Zukunft". Im Namen von Humboldts Bildungs-Idee des mündigen und selbständigen Bürgers geht es dem Autor um ein umfassendes digitales Bildungsangebot, in dem ,sämtliche existierenden und zukünftigen Aus-, Weiter- und Fortbildungsinhalte frei und jederzeit verfügbar sind“" (Meinel 2017). Diese Vision, die sich ausdrücklich auch auf das Lernen in Schulen und Hochschulen bezieht, folgt dem zentralen Gedanken, dass die in den digitalen Angeboten steckenden Inhalte unvermittelt von allen lernbegierigen Menschen abgerufen werden können. Worum es bei der Vorstellung geht, dass Bildung und Ausbildung als Problem autonomer Nutzung digitaler Möglichkeiten zu betrachten sind, lässt sich speziell am ausbildungsbezogenen Unternehmens-Diskurs ablesen. Er wird von der Erwartung bestimmt, dass Mitarbeiterinnen und Mitarbeiter selbst Verantwortung für ihre persönliche Weiterentwicklung übernehmen. So liest es sich in der 8. These eines Blogs, in dem von den Lernenden gefordert wird, „das eigene Lernen in die Hand zu nehmen, sich Lernziele $\mathrm{zu}$ setzen und das eigene Lernen zu steuern" (abc-tillmann.consulting in education de/blog/2015/12/16/10-thesen-zur-digitalisierung des lernens im unternehmen; abgerufen am 20.09.2016). Wenn das funktioniert, dann beschränkt sich die Aufgabe des Unternehmens künftig darauf, digitale Lernmaterialien anzubieten und sie - wie es der Vision von Meinel entspricht - in hoher Qualität verfügbar zu machen.

In diesem Trend läge es, wenn an den Hochschulen erstens die traditionellen Vorlesungen in eine digitalisierte Form gebracht und zweitens zusätzlich durch digitale Lernmaterialien ergänzt würden. Hinzu käme drittens, dass Studierende als Digital Natives das Lernen auf individuelle Eigenarbeit mit digitalen Geräten umstellen müssten.

Ersterem steht das traditionelle Festhalten am Vorlesungsprinzip entgegen. Eine kombinierte Realisierung des ersten und zweiten Punkts passt zudem nicht zur zögerlichen Annahme des Digitalen. Beim dritten Punkt schließlich würde ignoriert, dass es - wie hier herausgestellt - für ertragreiches Lernen darauf ankommt, dass die Lernenden in soziale Zusammenhänge eingebettet werden.

Was ist an Hochschulen in einer solchen Situation des didaktischen Attentismus zu tun? Einerseits gilt es das Potential des digitalen Lernens auszuschöpfen. Andererseits kann das neurobiologisch informierte Konzept dialogischen Lernens nicht in den Wind geschlagen werden. An dieser Stelle muss der Pionier eines intuitiv-pragmatischen und zugleich didaktisch

Lernens ins Spiel gebracht werden. Es ist der an der Fachhochschule Bielefeld arbeitende Physiker und Mathematiker Jörn Loviscach. 
Von ihm darf angenommen werden, dass er - zunächst ohne didaktisch mehr als nur

ausgebildet zu sein - seine Unzufriedenheit mit dem Lehrbetrieb an Hochschulen in improvisierte Angebote umsetzte und dann on the Job die Anleitung des Lernens lernte. Bei seiner Öffnung für digital unterstütztes Lernen halfen ihm seine Kenntnisse als Informatiker. Im Besonderen hatte er offensichtlich kein Problem damit, dass Lernen etwas mit jenen dialogischen Prinzipien zu tun hat, die er, am Lernerfolg der Studierenden interessiert, im Kontakt mit ihnen umzusetzen sucht.

Loviscach erzählte immer wieder, wie es dazu kam, dass er mit einfachster Aufnahmetechnik Lehr-Videos erstellte, die vom schon erwähnten OnlineUnternehmen Udacity sowie von YouTube übernommen wurden und - in technisch weiterentwickelter Form - eine riesige Resonanz hatten. Es ging ihm zunächst einfach darum, Studierenden zu helfen, die aus organisatorischen Gründen nicht an seinen Vorlesungen teilnehmen konnten. Wichtiger aber war, dass er dazu überging, überhaupt keine Vorlesungen mehr zu halten. Wie sich aus Vorträgen und Interviews ergibt, die sich im Internet nachlesen lassen, waren es seine Schwierigkeiten mit dem Vorlesungsprinzip - auch seine Zweifel am Lernertrag seiner online abrufbaren Videos -, die ihn zum „Aussteiger“ machten:

Loviscach produziert sorgfältig ausgearbeitete und immer wieder revidierte digitale Videos und Video-Serien mit elektronischem Tafelanschrieb (MikroTexte). Von den Studierenden wird erwartet, dass sie die Videos durcharbeiten, die den einzelnen Sitzungen - früher Vorlesungen - zugeordnet werden. $\mathrm{Zu}$ den jeweils aufgeworfenen Problemen kommt es in den Sitzungen zum Dialog mit dem Autor des jeweils vorbereiteten Materials. Es werden Fragen gestellt und beantwortet. Um zu überprüfen, ob die wichtigsten Inhalte verstanden wurden, werden auch Übungsaufgaben verteilt und in Gruppen bearbeitet.

Es ist leicht zu erkennen, dass der von Loviscach entwickelte und immer wieder praktizierte Lernansatz dem oben skizzierten und digital angereicherten Konzept dialogischen Lernens entspricht. Daher ist es nicht weiter überraschend, dass Loviscach seine Vorschläge problemlos mit der Etikette des zuerst von amerikanischen Didaktikern eingeführten Ansatzes des Inverted Classroom (auch: Flipped Classroom) versehen konnte.

Was hier zu den improvisierenden Anfängen von Jörn Loviscach berichtet wurde, gilt in noch viel stärkerem Maße für den Kölner Direktor des TU-Instituts für Werkstoffanwendung, Martin Bonnet. Wie sich einem Auftritt auf der Tagung „Diggi 17“ entnehmen lässt, hält er keine Vorlesungen mehr. ${ }^{64}$ Was er

64 Ein ausführlicher Bericht über Martin Bonnets Ausführungen, der hier zugrunde liegt, stammt von Larissa Holzki, „Lehre lieber ungewöhnlich“, in: Süddeutsche Zeitung, 18.12.2017. 
zur „Welt der Werkstoffe“ zu sagen hat, steht online auf YouTube und kann von den Studierenden zur Vorbereitung der Veranstaltungen mit Bonnet heruntergeladen werden. Nach dem Muster des Inverted Classroom, das er eher zufällig entdeckt hat, läuft seine Veranstaltung genauso $\mathrm{ab}$, wie es für Loviscach beschrieben wurde. Dabei zeigt sich Bonnets fehlendes Interesse an Didaktik daran, dass er fast entschuldigend an Kollegen gewendet betont, dass diese Art des Lernens speziell für ihn, einen Professor, aber möglicherweise nicht für andere Lehrpersonen geeignet sei.

Anders liegt der Fall des an der Heidelberger Pädagogischen Hochschule tätigen Christian Spinnagel. Er ist für Mathematikdidaktik zuständig und stimmt - auch in hier nicht zu belegenden Präsentationen seines Vorgehens in der Substanz mit dem Lernansatz von Loviscach überein.

Im Unterschied dazu ist Jürgen Handke ein Autor, der sich als professioneller Hochschuldidaktiker versteht. Seine Vorträge, Interviews und speziell seine Buch-Publikationen, auf die teilweise schon Bezug genommen wurde, setzen sich entschieden mit jenen Kräften auseinander, die an dem festzuhalten suchen, was hier obrigkeitliche Lehre genannt wird. Zugleich und hauptsächlich geht es ihm darum, das Konzept eines mit digitalen Materialien arbeitenden Lernens in der Praxis anzuwenden, es - soweit möglich - empirisch zu stützen und immer wieder auf den Prüfstand zu stellen. Es geht Handke um Präsenzlehre (hier: Präsenz-Lernen), in der sich die Studierenden einer dialogischen Konstellation stellen: Sie bringen die Kenntnisse, die sie sich in der Vorbereitung einzelner Sitzungen verschafft haben, in den Dialog mit der Lehrperson ein, die ihnen die Lernmaterialien vorgegeben hat.

Diejenigen, die das Lernen anleiten, sind gewissermaßen doppelt präsent. Sie stehen als Diskussionspartnerin und -partner zur Verfügung und sind zugleich dafür verantwortlich, dass sich die vorgegebenen digitalen Materialien - neben den auch im Portfolio liegenden traditionellen Medien - zur gemeinsamen Bearbeitung eignen. Im Besonderen ist es Handke wichtig, dass die Angebote der Technologie nicht unüberlegt eingesetzt werden. Vielmehr müssten umgekehrt die Pädagogik und Didaktik die Technologie antreiben (Handke 2014: 14, These 4 zur Digitalisierung). Konsequenterweise lehnt er die e $\quad$ Vorlesung als digitalisiertes Lernformat ab (Handke 2014: 95ff.). In neurobiologischer Perspektive kann dies ebenso wie seine Einschätzungen anderer digitaler Formate bekräftigt werden (Kap. V. 3.).

Was empirische Belege für die Wirksamkeit des Präsenz-Lernens angeht, so hat Handke - unter dem Dach des Inverted Classroom fortlaufend Fachkonferenzen angeregt, auf denen eine Vielzahl von Ideen, Projekten und praktischen Erfahrungen zur Diskussion gestellt werden (vgl. die Literaturangaben in Handkes Publikationen). Handke selbst hat eine sorgfältig konzipierte Analyse durchgeführt, die den Erfolg des Präsenz-Lernens 
überzeugend belegt. Beim Vergleich zwischen zwei Leistungsgruppen von Studierenden eines Masterkurses zeigte sich, dass diejenigen, die an der Präsenzveranstaltung teilgenommen hatten, sowohl in der Klausur als auch bei der Überprüfung einzelner Lerneinheiten deutlich besser abschnitten als diejenigen, die das Präsenzangebot nicht annahmen (Handke 2016, in: Großkurth/ Handke 2016: 27-40).

Da die traditionelle Vorlesung das Gegenmodell zum Präsenz-Lernen darstellt, ist es einigermaßen überraschend, dass Handke mit gewisser Sympathie die verbreiteten Bemühungen schildert, die Unzufriedenheit mit dem Frontalismus des Lernens abzufangen. Mittel hierzu sind Handouts, PowerPoint und Internetquellen (Handke 2014: 77f.). Auch werden Tutorien zur Vor- und Nachbereitung eingerichtet. Im Besonderen werden Studierende dazu aufgefordert, auch zwischendurch Fragen zu stellen. Das alles ändert indessen nichts daran, dass es sich hier um einen Veranstaltungstyp handelt, der durch die Präsenz einer gebieterischen Obrigkeit bestimmt wird, die es allein schon auf Grund ihrer Herausgehobenheit am Vortragspult unmöglich macht, mit den Studierenden gleichberechtigt zu kommunizieren.

Auffällig ist auch, dass Handke das Präsenz-Lernen relativiert, indem er es für möglich hält, die Teilnahme an den Präsenz-Veranstaltungen zu reduzieren. Das bringt ihn auf die Seite derjenigen Studierenden, die Präsenz für Zeitverschwendung halten und auf autonome Nutzung der digitalen Lernmaterialien schwören. Auch signalisiert er - möglicherweise ungewollt - sein Verständnis für das wachsende Angebot von Fern-Curricula, die mit wenig oder gar keiner Präsenz rechnen.

Hinter einer solchen Aufweichung des Präsenz-Gedankens darf man Handkes Einsatz für, so Handke, ,exzellente Online-Materialien“ und seinen berechtigten Stolz darauf vermuten, dass „ein Lehrvideo viele tausend Mal abgerufen wurde und sich die positiven Einschätzungen und Kommentare häufen ...“ (Handke 2016: 127). Hier gibt es Gemeinsamkeiten mit den einseitig auf die Umschaltung auf digitales Lernen konzentrierten Kollegen. Der Unterschied zu Handkes Grundposition liegt indessen darin, dass er - auf einer Linie auch mit Loviscach u. a. - ertragreiches Lernen in Nahkommunikation verankert und dieses Vorgehen durch elementare neurobiologische Erkenntnisse bestätigt wird.

Für alle digitalen Materialien gilt, dass in ihrer dialogischen Verarbeitung die Chance hohen Lernertrags liegt. Was auf der Dialog-Plattform den äußeren Anschein des unkontrollierten Diskussionsverlaufs hat, kann im Lernapparat der Studierenden zu vielspurigen und netzübergreifenden Lerneindrücken und damit zum Lernerfolg führen. 


\section{Wer dialogisches Präsenz-Lernen anleiten soll}

Es ist immer wieder überlegt und diskutiert worden, die Anleitung des Lernens an Hochschulen sogenannten Lehrprofessuren zu übertragen. Die Resonanz war indessen gering. An einigen wenigen Universitäten ausgewählter Bundesländer wurde eine verschwindend geringe Zahl solcher Stellen geschaffen und besetzt (Hilbrich u. a. 2014). Der Einführung von Lehrprofessuren wird immer noch das Humboldt'sche Prinzip einer als unverzichtbar geltenden Kopplung von Forschung und Lehre entgegengehalten.

Wenn es aber stimmt, dass dieses Prinzip zu einem Hochschulszenario gehört, in dem sich noch niemand über Vermittlungsformen des Lernens Gedanken machte (vgl. Kap. I.1.), so wäre ein Umdenken längst geboten. Zugleich läge es in der Konsequenz des begrenzten Lernertrags von Vorlesungen (vgl. Kap. IV.; auch Exkurs 1), die traditionell an das Vorlesungsprinzip gebundene Frage nach dem Charisma des Lehrpersonals an Hochschulen auf die Stufe dialogischen Lernens zu bringen.

Welches müssen diejenigen haben, die das Präsenz-Lernen anleiten sollen? Sie müssen dazu fähig sein und gelernt haben, zu den Lernenden in Nahkommunikation ein Vertrauensverhältnis aufzubauen. Auf dieser Grundlage müssen sie im lernzielbezogenen Dialog sprachlich, gestisch und mimisch so auftreten, dass die Lernenden zur Kommunikation herausgefordert werden (Attachment, Empathie, Entertainment). Nur so kann es im Lernapparat der Lernenden zur beschriebenen netzübergreifenden Vielspurigkeit der Lerneindrücke kommen, von der hoher Lernertrag abhängt.

Ein Kontrastvergleich mit der obrigkeitlichen Konstellation im Hörsaal macht deutlich, dass Vorlesende, wenn sie vom hohen Ross ihres Vorlesungspults auf die Dialogstufe heruntersteigen, ihre Kenntnisse nicht mehr von oben herab diktieren können. Im Sinne des auf Dialog angelegten Lernens muss der Lernstoff als stets bezweifelbar, befragbar und diskutierbar angeboten werden. In Verbindung mit persönlichen Grundtalenten bedarf es dazu einer hochschuldidaktischen Ausbildung, die gründliche Kenntnisse, den Einsatz sowohl traditioneller als auch digitaler Medien betreffend, einschließt. ${ }^{65}$

65 In den Handlungsempfehlungen, die eine Expertengruppe des Hochschulforums Digitalisierung in einem Zwischenbericht gab (Hochschulforum 2016), werden Vorschläge zur Veränderung der Personalstruktur vermieden. Es wird jedoch eine ,arbeitsteilige Erstellung und Durchführung digitaler Lehre“ angestrebt, die nicht entschieden genug deutlich macht, dass Lehrprofessorinnen und -professoren in Fragen der Digitalisierung zwar Unterstiutzung brauchen, den didaktisch reflektierten Einsatz digitaler Medien jedoch selbst beherrschen müssen. 
Wenn die Anleitung des Lernens mit der Aufforderung an die Lernenden verbunden ist, Eigeninitiative zu entwickeln, so heißt das immer auch, dass es dazu klarer Vorgaben bedarf. Hierfür müssen Fachausbildung und Fachkompetenzen mit den genannten nahkommunikativen Fähigkeiten verbunden sein.

In Übereinstimmung mit der traditionellen Koppelung von Forschung und Lehre heißt das aber umgekehrt auch, dass Forscherpersönlichkeiten ein zum Teil schwer beschreibbares "natürliches Charisma“ mitbringen, das sie zur Anleitung von Präsenzlehre geeignet macht. Dasselbe gilt für brillant Vorlesende, soweit sie die Fähigkeit entwickelt haben, von Fernkommunikation auf Nahkommunikation umzuschalten. Wer indessen an Studierenden nicht interessiert ist und nur vorlesen will, sollte sich in eine Forschungseinrichtung zurückziehen. In einer Vorausschau auf die Universität, wie er sie sich für die Zukunft wünscht, hat Jürgen Handke das fend beschrieben:

von Lehrprofessoren zutref-

„Die Professoren dieser Universität konnten sich zwischen Forschung und Lehre entscheiden: Mehr Forschung und weniger Lehre und umgekehrt, das Ganze durch entsprechende bzw. ausstattungsbezogene Anreizsysteme gefördert. Und wer sich ganz für die Lehre entschied, hatte keine Reputationseinbußen zu befürchten, musste sich aber permanenten Evaluationen stellen, wurde in einem berufsbegleitenden ständig von ausgewiesenen Experten in Sachen Lehr-/Lerntechnologien weitergebildet und musste schließlich im Gegensatz zu den reinen Forschungsprofessuren eine Habilitation zum Nachweis der, Venia Legendi nachweisen, die diesen Namen auch verdiente.“ (Handke 2014: 227)

\section{Die Chance der Hochschulen als Ort dialogischen Lernens}

Seit es das Internet und im weltweiten Netz die sogenannten sozialen Medien gibt, werden in lockeren Chats Informationen ausgetauscht. Wie auch in der traditionellen Alltags-Kommunikation geht es dabei nicht um die gezielte Beschaffung von Kenntnissen. Diese landen eher zufällig im Gedächtnis, versich oft aber ebenso schnell wie sie gekommen sind. Dasselbe gilt auch für die Benutzung des Netzes als Lexikon mit riesenhaftem Informationsspeicher. Es werden Antworten auf kurzfristig interessierende Quiz-Fragen gesucht.

Das Internet in seinen vielen Verästelungen ist jedoch auch eine Fundgrube für weitergehende und breitere Fragestellungen. Es bietet Wissensbestände an, die bislang ins Ressort von Forschung und Lehre der Hochschulen 
gehören. ${ }^{66}$ Je nach Bildungs- und Ausbildungsniveau hält das Netz für lernbegierige Menschen nahezu Bildungs- und Ausbildungs-Ressourcen bereit. Gefüllt wird dieses Lern-Reservoir von unzähligen außeruniversitären Einrichtungen und publizistischen Unternehmen staatlicher und privater Art. Was diese online verfügbar machen, kann „,hochschulfrei“ und in gewissem Umfang kostenfrei genutzt werden. Auch die Hochschulen selbst leisten ihren Beitrag zum außeruniversitären digitalen Bildungs- und Ausbildungs-Angebot. Neben kommerziellen Anbietern wie YouTube und Coursera bieten sie in besonders nachgefragten Wissensbereichen digitalisierte Vorlesungen und Moocs an (Kap. V.3.). Diese werden teilweise zu Werbezwecken

An den Hochschulen vorbei treffen sich - zumeist in naturwissenschaftlichen Fächern - Spezialisten in Internet-Foren. Sie informieren über die Ergebnisse ihrer Forschung, diskutieren sie und geben sie an Interessierte weiter. In solchen Foren und auf Internet-Plattformen wird auch schon breiter angelegte Projektarbeit geleistet, die von sogenannten Communities of Practice getragen wird und vermutlich großes Entwicklungspotential hat. Was bedeutet es für die Hochschulen, dass ihre bislang zentrale Funktion sowohl in der forschenden Produktion von Wissen als auch in der Verarbeitung und Weitergabe von Wissen stark eingeschränkt ist? Was bedeutet es insbesondere, dass die Hochschulen ihre bislang kaum angetastete Rolle als Prüfungsinstanzen und Gatekeeper

Karrieren mit anderen Institutionen zumindest teilen müssen?

Generell bedarf es nach wie vor einer Institution, die Lernaufgaben stellt und in der das Lernen, das auf bestimmte Lernfelder und mit ihnen verschränkte Berufsfelder gerichtet ist, durch geeignete Lehrpersonen angeleitet wird. Die falsche Umsetzung dieses generellen Befunds bestünde in einer bloßen Fortsetzung des Vorlesungslernens und anderer frontaler Lernformate. Hinzu käme das Zugeständnis an die Studierenden, die verfügbaren digitalen Lernmöglichkeiten hochschulextern autonom zu nutzen. Hinter dieser Option stünde der traditionelle Anspruch, dass fachlich exzellente Vorlesende, die mit Charisma ausgestattet sind, Synthesen des von ihnen beherrschten Wissens den Studierenden „beibringen“ können, generös ergänzt um ein Bekenntnis zum digitalen Fortschritt.

Die richtige Umsetzung beruht zum einen auf der neurobiologisch begründeten Einsicht, dass Vorlesungslernen - Impuls-Vorlesungen ausgenommen (vgl. bes. die Zusammenfassung von Kap. IV.) - nur geringen Lernertrag lie- 
fert. Sie stützt sich zum andern auf den in der Auseinandersetzung mit dem Einsatz digitaler Lernmaterialien geführten Nachweis, dass das Ergebnis ihrer autonomen Verarbeitung nicht über die Stufe spurenarmer neuronaler Lerneindrücke hinausreicht (Kap. V.3.). Die richtige Umsetzung der generellen Antwort besteht dann in der alles andere umschließenden Anwendung des Konzepts dialogischen Lernens. Die Chance der Hochschulen liegt darin, dass sie - neben ihrer Stellung als Institution der Forschung und unter Nutzung aller verfügbaren Lernmaterialien - den Studierenden einen Lernort bieten, an dem sie in Nahkommunikation mit Lehrpersonen ertragreich lernen, weil sie gehirngerecht lernen. 



\section{Schlussbilanz}

\section{Obrigkeitliche Lern-Konstellation und dialogische Lern-Konstellation}

Ein neurobiologisch informiertes und dadurch didaktisch inspiriertes Lernverständnis, das auf möglichst hohen Lernertrag zielt, bezieht sich auf individuell und kommunikativ vermittelte Lernprozesse. Im Kern bestehen sie darin, dass über die Sinnesorgane vielfach Lernimpulse aufgenommen und neuronal verarbeitet werden. In einer obrigkeitlichen Lern-Konstellation kommen diese Impulse aus vertikaler Fernkommunikation. Diese ist durch eine Dominanz der Lehrpersonen (vgl. Anm.7) gekennzeichnet. Für Eigenaktivität der Lernenden ist wenig Platz. Dem steht eine dialogische Lern-Konstellation gegenüber, bei der in horizontaler Nahkommunikation auch Impulse der Lernenden in den Lernprozess eingehen.

In beiden Konstellationen besteht der Lernvorgang darin, dass Lernimpulse in Erinnerungen umgesetzt werden und im Gedächtnis Fakten-Wissen, konzeptuelles und prozedurales Wissen aufgezeichnet werden.

\section{Ertragarme obrigkeitlicher Lehre an bundesrepublikanischen Hochschulen}

Die Hochschulen im Nachkriegsdeutschland stehen in der Tradition obrigkeitlicher Lehre. Die allgemeine Lern-Konstellation ist dadurch gekennzeichnet, dass es zwischen der großen Mehrheit der Studierenden und den Lehrpersonen eine große Distanz gibt. Rituale einer altehrwürdigen Institution einerseits, leistungsorientierte andererseits, schaffen symbolische Einbettungen, in denen sich Studierende winzig vorkommen. Neuronale Strukturen und Modulatoren sorgen dafür, dass die große Mehrheit der Studierenden ruhiggestellt wird. $\mathrm{Zu}$ diesem Arrangement gehört ihre Bereitschaft, in einem didaktischen Vakuum zu lernen und sich mit wenig Betreuung und Beratung

Nur wenige Einzelgängerinnen und Einzelgänger sind nicht bereit, sich dieser Mehrheit zu fügen. Eine privilegierte Minderheit praktiziert nachsichtige Unzufriedenheit mit den Lehrpersonen, weil sie auf diese Weise ihr Kontakt-Privileg absichern kann.

Die spezielle Lern-Konstellation im Vorlesungsbetrieb fügt sich passgenau in die allgemeine Konstellation ein. Zwischen Studierenden und Vorlesenden liegt im Hörsaal eine große Distanz. Es kommt nicht zur Nahkommunikation. 
Insoweit sind Vorlesungen symptomatisch für eine obrigkeitliche Konstellation, in der Lehrpersonen dominieren. Vorlesungen dämpfen nicht nur die „natürliche Neugier", die Studierende mitbringen. Im fernkommunikativ angelegten Lernprozess ist der Lernertrag gering, weil die neuronalen Strukturen des Kurzzeit- und Langzeitgedächtnisses den Vorlesenden nicht entgegenkommen. Beträchtliche Teile der Fülle des dargebotenen Lehrstoffs werden einerseits erst gar nicht aufgenommen. Andererseits bleibt es in Hörsälen bei einem Niveau der neuronalen Erregung von Lernenden, das in der Regel zu niedrig ist, um einen ohnehin reduzierten Input ins Langzeitgedächtnis zu befördern. Damit geht der Anspruch auf ertragreiche Wissensvermittlung weitgehend ins Leere.

\section{Wie in dialogischer Lern-Konstellation hoher Lernertrag entsteht}

ausgestaltetes dialogisches Lernen nach dem Prinzip des Inverted Classroom (umgekehrtes Klassenzimmer) ist geeignet, aus ertragarmer obrigkeitlicher Lehre herauszuführen. Sorgfältig ausgewählte - sowohl traditionelle als auch digitale - Lernmaterialien bilden die Grundlage eines nahkommunikativen Austauschs zwischen Lehrpersonen und Lernenden. Sie treffen in der Absicht aufeinander, sich gegenseitig herauszufordern und anzutreiben. Dies ist die Voraussetzung dafür, dass es im neuronalen Apparat der Lernenden zu Aufruhr (Attachment, Empathie, Rollenspiel) und auf synaptischer Stufe zu netzübergreifender Vielspurigkeit kommt. Von ihr ist hoher Lernertrag abhängig.

\section{Zum pädagogisch-didaktischen Mehrwert neurobiologisch informierten Lernens}

\begin{tabular}{|r|l|}
\hline $\begin{array}{r}\text { Neurobiologisch begrün- } \\
\text { dete Ablehnung von: }\end{array}$ & $\begin{array}{l}\text { obrigkeitlicher Lehre } \\
\text { speziell: Ablehnung von (Massen-) Vorlesungen = ertragar- } \\
\text { mes Lernen }\end{array}$ \\
\hline $\begin{array}{r}\text { Neurobiologisch begrün- } \\
\text { dete Anerkennung von: }\end{array}$ & $\begin{array}{l}\text { intuitiv-evidenzbasiertem Konzept dialogischen Lernens } \\
\text { (v. a.: Inverted-Classroom) }\end{array}$ \\
\hline $\begin{array}{r}\text { Neurobiologisch begrün- } \\
\text { dete Ausarbeitung von: }\end{array}$ & $\begin{array}{l}\text { differenziertem Konzept dialogischen Lernens = ertragrei- } \\
\text { ches Lernen (wg. netzübergreifender Vielspurigkeit des im } \\
\text { Gehirn erzeugten Aufruhrs) }\end{array}$ \\
\hline $\begin{array}{r}\text { Neurobiologisch begrün- } \\
\text { dete Unterstützung von: }\end{array}$ & $\begin{array}{l}\text { Konzept dialogischen Präsenz-Lernens mit digitalen Mate- } \\
\text { rialien = ertragreiches Lernen (wg. netzübergreifender Viel- } \\
\text { spurigkeit des im Gehirn erzeugten Aufruhrs) }\end{array}$ \\
\hline $\begin{array}{r}\text { Neurobiologisch begrün- } \\
\text { dete Empfehlung von: }\end{array}$ & verändertem Profil von Lehrpersonen \\
\hline
\end{tabular}




\section{Exkurs 1}

\section{Lernen konzeptionellen Wissens - Neuro-motivationale Voraussetzungen}

Es ist eine Alltagserfahrung, dass sich Kinder ebenso wie Erwachsene durch einzelne oder auch wiederholte Beobachtungen und Erlebnisse beeindruckt zeigen. Jemand, der ein Schachspiel verfolgt oder im Konzert eine Solo-Geigerin erlebt, sagt spontan: „Das reizt mich.“ Neurobiologisch betrachtet heißt das, dass die neuronalen Areale, die für Schach- und Geigenspiel zentral und peripher zuständig sind, gereizt werden. Insoweit stimmt der im Gehirn ungesteuert erzeugte Effekt mit seiner verbalen Präsentation überein. Diese Übersetzung eines automatischen neuronalen Vorgangs lässt sich auch im Begriff der Motivation fassen, der vielfach und zumeist in unscharfer Bedeutung verwendet wird. In einem präzisen neurobiologischen Verständnis lässt sich für das beispielhaft ausgewählte Schach- oder Geigenspiel sagen: Sie motivieren in dem Sinne, dass bestimmte Neuronengruppen bewegt werden.

Auf einer anspruchsvolleren Stufe der Reaktion auf Schach- und Geigenspiel, auf der im Stirnhirn angelegte Möglichkeiten der Steuerung ins Spiel kommen, kann es dann zu der Reaktion kommen: „Das will ich lernen.“ Ich will selbst Schach oder als Mitglied eines Orchesters Geige spielen können. Neurobiologisch betrachtet heißt das, dass an die Stelle ungesteuerter Bewegung die gezielte Schaffung neuro-motivationaler Voraussetzungen für Schach- und Geigenspiel treten können. Gelingt dies, so hat jemand Schach oder Geige gelernt.

Wer als Kind in einem Elternhaus aufgewachsen ist, in dem Schach gespielt und Musik gemacht wurde, bringt solche Voraussetzungen bis zu einem bestimmten Grade schon mit. Lernen läuft in diesem Falle darauf hinaus, dass schon bestehende neuro-motivationale Voraussetzungen gefestigt und ausgebaut werden. Bei hochschulischem Lernen geht es indessen im Regelfall um die Erschließung bislang eher unbekannter Lernbereiche. Das gilt im Besonderen für Lernprozesse in den Naturwissenschaften.

Dies ist der Erfahrungsbereich des amerikanischen Physikers Carl Wieman, der - verkürzend zusammengefasst - zeigen kann, dass die erfolgreiche Vermittlung von Einzelinformationen nur gelingt, wenn Studierende in die Konzepte eingeführt werden, von denen diese Informationen zusammengehalten werden. Wer etwa im Lernbereich der Elektrizität informiert werden will, so argumentiert Wieman, muss vermittelt bekommen, auf welche Weise über das Thema nachgedacht wird. Wie sehen die Begriffe, die ihnen zugrunde liegenden abstrakten Modelle und die Gesetze aus, auf die Physikerinnen und Physiker hinarbeiten und die sie experimentell zu beweisen und anzuwenden 
suchen. Es geht vorrangig um die Vermittlung eines mental framework und neurobiologisch betrachtet - darum, breite neuro-motivationale Voraussetzungen dafür zu schaffen, dass physikalisches Wissen zusammenhängend aufgenommen werden kann.

Wieman erhielt 2001 den Nobel-Preis in Physik und wurde danach zum Hochschuldidaktiker. Ihm kam der Verdacht, dass die von ihm selbst und anderen praktizierte Vermittlung naturwissenschaftlicher Kenntnisse in höchst unzulänglicher Form erfolgt. Das gilt im Besonderen für die traditionelle Form der Vorlesung, in der Anfängerinnen und Anfänger in die Physik eingeführt werden sollen. Zur Überprüfung seiner Vermutungen leitete Wieman - an der Universität in Boulder (Colorado) und an der University of British Columbia (UBS) - umfangreiche Forschungen an, zuletzt ein Projekt, dessen Ergebnisse in der renommierten Zeitschrift Science (13.05. 2011: 862-864) veröffentlicht wurden. Zum einen bestätigte sich in immer neuen Befragungen von Studierenden, dass der Lernertrag frontaler Vorlesungen herkömmlichen Typs erschreckend gering war. Informationen, unmittelbar nach Vorlesungen erfragt, wurden nur in einer Größenordnung von ungefähr 20\% behalten. Im Kontrast dazu ergab sich zum anderen, dass sich der Lernertrag in spektakulärer Weise steigern ließ, wenn Lernformen gewählt wurden, die für gegenseitiges Feedback zwischen Lehrpersonen und Lernenden sorgten. Es erwies sich, dass von solchen personal response systems im Besonderen jenes konzeptionelle Wissen abhängig ist, das selbst wiederum die Aufnahme und Verarbeitung von Faktenwissen ermöglicht. (Im Internet sind Reden abrufbar, in denen Wieman seine jeweils neuesten Erkenntnisse immer wieder zusammenfasst.)

Die Einsichten und Anregungen Wiemans werden im Folgenden aufgenommen, weil sie geeignet sind, die Analysen des III. und IV. Kapitels zu vervollständigen. Spezielle Fragen nach der Vermittlung konzeptueller Kenntnisse werden hier vernachlässigt. Es soll vielmehr plausibel gemacht werden, dass faktische Informationen nur dann eine Chance haben, bei den Studierenden anzukommen, wenn es gelingt, deren neuronale Aufnahme- und Verarbeitungskapazität breit auszubilden.

Was das inhaltlich bedeutet, lässt sich im Anschluss an das bekannte Phänomen der selektiven Wahrnehmung am einfachen Beispiel der sozialwissenschaftlichen Vergleichsbetrachtung zeigen, einem unverzichtbaren Instrument der Analyse, das fachübergreifend eingesetzt wird. Ergänzend zur auf Wieman gestützten Argumentation werden einige Schlaglichter auf die in sozialwissenschaftlichen Spezialbereichen behandelte Ideengeschichte geworfen.

Was die breite Ausgestaltung der Aufnahme- und Verarbeitungskapazität der Studierenden neuro-motivational voraussetzt, lässt sich anhand von Grundkenntnissen über das Jagen von Tieren illustrieren. Wer zu erfolgreichen Beutezügen in der Lage sein soll, muss das Jagdverhalten in dem Sinne gelernt 
haben, dass die für dieses zuständigen neuronalen Netzwerke umfassend ausgeformt wurden. Diese Netzwerke unterscheiden sich in ihrem grundlegenden Design nicht vom menschlichen Gehirn. Daher ist erfolgreiches menschliches Jagdverhalten unterschiedlicher Art, das hier als Jagd nach Vorlesungs-Wissen untersucht wird, von entsprechend breiten neuro-motivationalen Grundlagen abhängig.

Um das konzeptionelle Niveau sozialwissenschaftlicher Vergleichsbetrachtung zu bestimmen, kann von Alltags-Diskussionen ausgegangen werden, die von dem Einwand beherrscht werden: „Das kannst du doch damit nicht vergleichen“. Es heißt dann beispielsweise: „Das sind doch zwei Paar Stiefel“, oder auch: „Man kann doch nicht Äpfel mit Birnen vergleichen“. Diese intuitive Abwehr des vergleichenden Blicks hat hauptsächlich zwei Gründe, die auch miteinander kombiniert sein können.

Am Beispiel des Themas Krieg kann dies illustriert werden: Die Ablehnung von Vergleichen liegt zum einen daran, dass Diskussionsteilnehmende befürchten, schon die bloße Beschäftigung mit Vergleichsobjekten lege sie auf normative Positionen fest, die sie ablehnen. Wenn Kriegsgegnerinnen und -gegner aufgefordert werden, aktuelle Kriege mit dem 1. oder 2. Weltkrieg zu vergleichen, weigern sie sich mit der stillschweigenden Begründung: Wenn wir uns auf den Vergleich aktueller Kriege mit früheren Kriegen einlassen, dann fügen wir uns der Unausweichlichkeit des Kriegshandwerks als Mittel der Politik. Die Vergleichsobjekte beweisen es. Daher müssen wir uns der Vergleichsbetrachtung entziehen.

Der zweite Ablehnungsgrund hat mit spektakulären Merkmalen der angebotenen Vergleichsobjekte zu tun. Beim Thema Krieg unterscheidet sich beispielsweise die Größenordnung der aktuellen Kriege sehr offensichtlich von derjenigen des 1. und 2. Weltkriegs. Bei spontan ablehnenden Reaktionen, die sich auf offensichtliche punktuelle Unterschiede beziehen und weitere Kontrastvergleiche erscheinen lassen, werden dann aber nicht nur diese, sondern zugleich auch Annäherungsvergleiche abgelehnt: Kriege völlig unterschiedlicher Größenordnung können doch nichts gemeinsam haben.

Eine Variante der Zurückweisungen annähernder Vergleichsbetrachtungen steckt in dem Einwand: „Dieser Vergleich hinkt.“ Damit ist gemeint, dass die verglichenen Objekte nicht identisch sein können. Diese ebenso richtige wie banale Einsicht verstellt den Blick darauf, dass es bei Annäherungsvergleichen allenfalls darum gehen kann, Ähnlichkeiten der Objekte - nach der präzisen Bezeichnung des Kybernetikers Karl Deutsch analogical properties - zu entdecken.

Demnach zeigt sich ein alltagsweltlicher Common Sense, der einerseits die Vergleichsbetrachtung aus normativen Gründen ausschließt: Diskussionsteilnehmende mögen entweder keine Äpfel oder keine Birnen und lassen sich 
daher nicht auf die vergleichende Festlegung auf die Frucht ein, die ihnen zuwider ist. Derselbe Common Sense besteht andererseits darin, dass spektakuläre Unterschiede zwischen den Vergleichsobjekten (Äpfel sind der Form nach eher rund und Birnen eher ,wellig“) weitere Kontrastvergleiche nicht lohnend erscheinen lassen. Zugleich sieht es so aus, als könne man Äpfel und Birnen einander nicht annähern.

Von diesem Hintergrund lässt sich das konzeptionelle Niveau der Sozialwissenschaften abheben. Sie bringen ein mental framework ins Spiel, das eine kombinierte Ausschöpfung sowohl der Kontrast- als auch der Annäherungs-Perspektive erlaubt: Auf diese Weise wird es möglich, die je besondere Mischung von Merkmalen herauszuarbeiten, durch die sich die verglichenen Objekte je einzeln auszeichnen. Formen, Größen, Farben, Geschmacksrichtungen, Nährstoffe und Verwendungsmöglichkeiten von Äpfeln lassen sich im vielseitigen Vergleich ebenso präzise ermitteln wie entsprechende Eigenschaften von Birnen. Für das Thema Krieg bedeutet das, dass aktuelle Kriege im Vergleich etwa zum 1. und 2. Weltkrieg nach je speziellen Kriterien analysiert und auch bewertet werden können. Dabei handelt es sich vor allem um Gesichtspunkte der ökonomischen und institutionellen Einbettungen, der ideologisch-religiösen Begründungen und Verursachungen sowie der waffentechnischen und gewaltförmigen Durchführung.

Überträgt man diese Einsichten auf den Vorlesungsbetrieb, so kommt der auf hohem konzeptionellem Niveau - beispielsweise mit Hilfe eines differenzierten Instrumentariums der Vergleichsbetrachtung - aufbereitete Stoff bei Lernenden nicht an, die sich als Zuhörende auf der Stufe selektiver Wahrnehmung Dies kann beispielsweise mit dem politischen Systemvergleich illustriert werden. Bedienen sich Vorlesende eines differenzierten analytischen Vergleichs-Instrumentariums, um ausgewählte Demokratien und Diktaturen in ihren je speziellen Merkmalen zu erfassen, so haben Lernende der Common-Sense-Stufe folgende Schwierigkeit: Da beide Vergleichsobjekte bei Betrachtung völlig gegensätzlich sind und daher einander nicht angenähert (das heißt nicht miteinander verglichen) werden dürfen, fehlt ihnen das Verständnis für die differenziert belegbare These, dass das Regiment von Regierungschefs westlicher Demokratien diktatorische Züge haben kann.

Beispielsweise in der fächerübergreifend arbeitenden Ideengeschichte, einem etablierten Lerngegenstand, sind die Gedankengebäude, die von Ausnahmedenkern unterschiedlicher zeitlicher Epochen errichtet wurden, den Lernenden - zuweilen auch denen, die das Lernen anleiten, - oft fremd. Nur der sorgfältig angeleitete Einstieg in zeitliche Rahmenbedingungen schützt vor Missverständnissen. Wer Klausuren einschlägiger Vorlesungen korrigiert hat, weiß, was der aktuelle Alltagsverstand aus zeitgebundenen Denkweisen macht. Man denke etwa an „wilde Interpretationen“ des platonischen Höhlengleich- 
nisses. Zeitübergreifend ist mangelndes Verständnis für Argumentationslinien oft darin begründet, dass Lernende nicht mit Gedankenexperimenten vertraut sind. Niemand kann ohne intensive Hilfestellung verstehen, was es (beispielsweise für Jean-Jacques Rousseau) heißt, einen Naturzustand als rückwärts gewandte Fiktion zu konstruieren, um dieser Konjektur dann eine Zukunftsvision hinzuzufügen, die als Vertrag konzipiert ist, den einzelne Bürger mit sich selbst (!) abschließen. Solche Gedankengänge sind dem Alltagsverstand der Studierenden nicht zugänglich. Auch enthusiastische Vorleserinnen und Vorleser (vgl. Kapitel IV 1./2.), können nichts am Unverständnis von Studierenden ändern, die nicht in die Besonderheiten spezieller Denkstile eingeweiht sind.

Versteht man Studierende als Jägerinnen und Jäger und den Lernertrag, auf den sie als Lernende hinarbeiten, als Beute, so kann ein solcher Vergleich Grundkenntnisse der Primatenforschung und speziell neurowissenschaftliche Kenntnisse heranziehen. Die zu vergleichenden Beutezüge sind eine Mischung aus ungesteuerten und gesteuerten Mechanismen, die im Falle studentischen Lernens keinen motorischen Zugriff auf die Beute enthalten. Gemeinsam ist ihnen, dass sie Aufmerksamkeit (attention) für Informationen hervorbringen und für ihre Aufnahme und Verarbeitung (perception) sorgen. Die gemeinsame Lernkomponente besteht darin, dass der Mechanismus der Verarbeitung von Impulsen den Aufbau von Erinnerungen einschließt. Die Gedächtnissysteme, in denen diese Erinnerungen aufbewahrt werden, sind ein Kernstück der zu Beginn dieses Exkurses beschriebenen neuro-motivationalen Voraussetzungen, auf die sich immer wieder neue Beutezüge stützen können.

Wie sehen diese Grundlagen aus? (Vgl. neben Einführungsliteratur bes. Kandel et al. (Ed.) 2000: 334) Die erfolgreiche Jagd von Primaten nach Beute hängt von Hungergefühlen ab. Diese werden vom Blutzuckerspiegel bestimmt und durch sensorische sowie modulatorische Netze im Hypothalamus Hinzu kommen Verbindungen zu modulatorischen Systemen, die in anderen Gehirnarealen liegen und miteinander verknüpft auf das Hungergefühl einwirken. $\mathrm{Ob}$ und bis zu welchem Grade dieses gefördert wird, hängt vor allem davon ab, welche Impulse von neuronalen Arealen aufgenommen werden, die hauptsächlich für Beobachtungen, für Höreindrücke und für Düfte zuständig sind. Die kognitiven Prozesse, die sich aus dem Zusammenwirken dieser Areale ergeben, bedürfen zusätzlicher Unterstützung aus den Netzteilen, die für Belohnungserwartungen und für Belohnung zuständig sind. Gefragt sind zudem die corticalen und subcorticalen Systeme, die motorische Wirksamkeit entfalten und das jagende Tier dazu drängen, sich der Beute zu nähern.

Um diese grobe Skizze der neuro-motivationalen Voraussetzungen tierischen Jagdverhaltens auf Prozesse des Lernens in Hörsälen und speziell auf den Aufbau konzeptionellen Wissens zu übertragen, ist zu beachten, dass der spezielle Akt des Ergreifens der Beute - es ist der von Lernenden erstrebte 
Lernertrag - generell kein lokaler und lokal fundierter Vorgang ist. Vielmehr kommt es darauf an, dass Gehirnareale vielfältiger Zuständigkeit und in vielschichtigen Netzen miteinander verknüpft, sich so breit organisieren und in umfassender Weise ,anstecken“ lassen, dass aus ihrem spannungsreichen $\mathrm{Zu}$ sammenwirken jene generellen Lernbereitschaften entstehen, die Wieman mit dem Gedanken des mental framework verbindet.

Mit Wieman kann argumentiert werden, dass Hochschulen, die spezielles Wissen vermitteln wollen, dafür sorgen müssen, dass in den Gehirnen der Lernenden dieses neuronal unterbaute mental framework entsteht. Wird es nicht planvoll entwickelt und ausgeformt, so können stückweise vorgetragene Informationen nur zum kleinen Teil aufgenommen und im Gedächtnis aufbewahrt werden. Nur auf dieser Ausgangsbasis kann erwartet werden, dass sich die in den Köpfen der Lehrpersonen unterschiedlicher Fächer steckenden Faktenkenntnisse an die Studierenden vermitteln lassen.

Was die Wege der Vermittlung angeht, so verweist Wiemans Schlüsselbegriff der deliberative practice darauf, dass seine Reformvorschläge auf der Linie der Lernszenerien liegen, die im V. Kapitel beschrieben werden. Speziell der Einstieg in Denkweisen und Denkstile ist darauf angewiesen, dass (zunächst) Unverständliches, Ungereimtes und Unfassbares auf unnachgiebige Fragen von Lernenden stößt, die durch entgegenkommende Lehrpersonen geduldig bearbeitet werden. Dabei kann neueste Kommunikationstechnik, wie sie etwa Wieman als empirischer Naturwissenschaftler vorschlägt und praktiziert (vgl. Clicker-System), durchaus hilfreich sein. Die auf persönliche Nähe angewiesene Eindringlichkeit der Kontakte wird sie freilich nicht ersetzen können.

\section{Exkurs 2}

\section{Vorlesungen als Varianten frontaler Programm-Reden in Politik und Gesellschaft - neurobiologisch informierte Vergleichsbetrachtungen}

Vorlesungen, sowohl Impuls- als auch Instruktions-Vorlesungen, sind Varianten eines übergreifenden Typus von Reden, die frontale Programm-Reden genannt werden können: Herausgehobene einzelne Rednerinnen und Redner versuchen einem ausgewählten Publikum bestimmte Botschaften nahezubringen und es in dem Sinne zum Lernen anzuleiten, dass es diese Informationen zur orientierenden Grundlage seines Handeln macht. In neurobiologischer Sicht wurde im II. (II.1./2.) und IV. Kapitel (IV.3.) gezeigt, dass es für den Ertrag von Vorlesungen darauf ankommt, dass sie auf die Voraussetzungen ,neuronaler 
Partizipation" abgestimmt sind, die das studentische Publikum mitbringt. Das gilt entsprechend für beliebige Reden und ihr Publikum, genereller noch für die Wirkung, die beliebige Reize bei ihren Adressaten erzielen.

Im Folgenden wird zum Ersten am Beispiel von Forschungen der sogenannten Neuroästhetik erläutert, wie Werke der bildenden Kunst, Bilder oder Skulpturen, beschaffen sein müssen, wenn sie von den Adressaten in großer Intensität und Breite aufgenommen und vor allem im emotionalen Gedächtnis verankert werden sollen (1.). Hier bestätigt sich auf einem Umweg, dass Impuls-Vorlesungen - dank ihrer Vieldeutigkeit - neuronale Durchschlagskraft besitzen.

Zum Zweiten lässt sich illustrieren, dass Impuls-Reden multipler Ausstrahlung, die außerhalb von Hörsälen gehalten werden, eine analoge Durchschlagskraft haben (2.). Am Beispiel ausgewählter politischer Programm-Reden Wahlkampf- und Parteitags-Reden - lässt sich zeigen, welche Kriterien erfüllt sein müssen, wenn sie beim Publikum ,ankommen“ sollen. In der Absicht, das Erregungspotential und damit das Potential der neuronalen Eingängigkeit von Impuls-Reden zu überprüfen, werden die politischen Programm-Reden einerseits mit dem Redeformat der Predigt verglichen. Andererseits wird auf Merkmale hingewiesen, die sich bei sowohl religiös als auch säkular geprägten Trauerreden beobachten lassen.

Unabhängig vom politischen und gesellschaftlichen Feld, in dem programmatische Reden gehalten werden, muss der Persönlichkeitsfaktor einbezogen werden: Am Beispiel „multipler Personen“ zeigt sich, worauf die Wirksamkeit von Charisma beruht (vgl. Kapitel IV.1., 2.) und was es umgekehrt bedeutet, wenn uncharismatische Personen reden.

Schließlich wird auf Instruktions-Reden eingegangen, die außerhalb von Hörsälen gehalten werden (3.). Dabei lässt sich plausibel machen, dass ihre neuronale Eingängigkeit tendenziell genauso unbefriedigend ist, wie dies in Kapitel IV.3. für Instruktions-Vorlesungen gezeigt wurde. Eingang sie nur im Ausnahmefall, wenn sie an vorhandene Erinnerungs-/Wissensbestände des Langzeitgedächtnisses anschließen können (3.).

\section{Erkenntnisse der Neuroästhetik - Kunstwerke mit hohem Erregungspotential}

Im Gegensatz zu den auf Instruktion zielenden (Massen-)Vorlesungen können Impuls-Vorlesungen unter bestimmten Voraussetzungen das Bedürfnis nach vertieften Lernerlebnissen befriedigen. Im preußischen Reform-Milieu der Wende vom 18. zum 19. Jahrhundert in eindrucksvoller Weise praktiziert (Fichte, Schleiermacher, Humboldt), ermöglichen sie begabten Vorleserinnen und Vorlesern - so wurde argumentiert -, das ihres Faches einprägsam zu vermitteln (vgl. Kapitel IV., Zusammenfassung). 
In neurobiologischer Sicht lässt sich dies durch grundlegende Einsichten der sogenannten Neuroästhetik stützen (vgl. bes. Zeki 2010). Sie geht zum einen davon aus, dass es in menschlichen Gehirnen angeborene Dispositionen dafür gibt, spezielle Reize - beispielsweise Lichtwellen, die von Flächen werden, - als Farbeindrücke zu verarbeiten. Zum anderen gibt es erworbene Dispositionen: Sie werden durch Erfahrungen erzeugt und durch immer wieder neu hinzukommende Erfahrungswerte fortlaufend verändert. Auf diese Weise bildet sich ein neuronales Verarbeitungspotential heraus, das einströmende Reize vielfach und variabel interpretierbar macht.

Das bedeutet, dass Kunstwerke, Bilder und Skulpturen, die in ihrer gesamten Komposition und im Detail ihrer Ausgestaltung eine breite Palette von Eindrücken vermitteln, in ihrer Vieldeutigkeit (Ambiguität) vielseitige (ambigue) Interpretationen herausfordern. Neben dem limbischen System, das für die emotionale Seite dieser Interpretationen zuständig ist, wird vor allem der orbitofrontale Cortex des Stirnhirns angesprochen, ein wichtiger Teil des auch subkortikal angelegten Belohnungssystems (Zeki 2010: 65ff.).

Zeki illustriert dies mit dem Gemälde „Das Mädchen mit dem Perlenohrring“ des niederländischen Malers Jan Vermeer, das ,auf dem Gemälde ... zugleich einladend und distanziert, voller erotischer Spannung und keusch, vorwurfsvoll und doch erfreut" wirkt (Zeki 2010: 95). Welche der möglichen Interpretationen sich durchsetzt, wird in den Gehirnen der Betrachtenden entschieden.

Anstöße zu vielfältiger und auch widersprüchlicher neuronaler Verarbeitung ambiguer Impulse geben insbesondere auch Skulpturen. Zeki verweist vor allem auf Michelangelo, der seine Figuren oft unvollendet ließ, so dass die Betrachtenden zu fortsetzender Eigentätigkeit ermuntert werden (Zeki 2010: 97ff.). In jedem Falle bieten Kunstschaffende Anlässe, die den Konsumenten ,neuronale Partizipation“ abverlangen, indem sie dazu aufgefordert werden, ihre je individuellen Möglichkeiten der Interpretation auszuschöpfen.

Was den Vergleich mit den Impuls-Vorlesungen angeht, so entspricht die Fülle ihrer Sprach-, Hör- und gestisch-mimischen Bilder der Ambiguität von Kunstwerken. Das erworbene neuronale Potential wird in großer Tiefe und Breite herausgefordert. Im Besonderen wird jener emotionale Aufruhr erzeugt, der erwiesenermaßen für die Schaffung von Erinnerung/Wissen unverzichtbar ist. Zugleich leuchtet es ein, dass Instruktions-Vorlesungen, in der Tendenz darauf gerichtet, geschlossene, abgerundete und komplette Informationen zu liefern, eben diesen erinnerungsbildenden Aufruhr nicht zustande bringen. Es fehlt ihnen das Erregungspotential von Kunstwerken, denen Impuls-Vorlesungen in ihrer vielfältigen Interpretationsoffenheit nahekommen. 


\section{Politische und unpolitische Impuls-Reden - „Redewerke“ mit hohem Erregungspotential}

Politische Programm-Reden sind in politischen Systemen mit demokratischem Anspruch immer Wahlkampf-Reden. Aber auch in weniger demokratischen Systemen geht es darum, dass sich politische Führer durch Ansprachen der Zustimmung ihres Volkes zu versichern suchen. Beispielhaft können einige Reden angeführt werden, von denen angenommen werden darf, dass die Grundzüge ihres Inhalts und das ihrer Autoren den Leserinnen und Lesern vertraut sind.

Die Wahlkampf-Reden Konrad Adenauers, des ersten Kanzlers der Bundesrepublik Deutschland, zeichnen sich dadurch aus, dass in relativ kurzer Zeit relativ wenig Inhalt präsentiert wird. Intuitiv berücksichtigt Adenauer, dass die Aufnahmefähigkeit des sogenannten Kurzzeitgedächtnisses sowohl zeitlich als auch inhaltlich beschränkt sind (vgl. Kap. II.1., 2.). Adenauer durfte annehmen, dass im Langzeitgedächtnis sowohl seiner Wahlbürger als auch im Gedächtnis des immer zugleich angesprochenen Auslandspublikums die noch nicht lange zurückliegenden Nazi-Verbrechen und die mit ihnen verbundene Last der generellen deutschen Verantwortung verankert waren. Es ist dieser Faktenbezug, der ausdrücklich oder stillschweigend mit einer Schlussfolgerung verbunden wird: Aus seiner verbrecherischen Vergangenheit muss Deutschland dadurch heraus, dass es sich als verlässlicher Bündnispartner des Westens erweist. Mit diesem in wenigen Varianten immer wieder formulierten doppelten Kernstück seiner Reden fand Adenauer beim doppelten Publikum Gehör, weil sie an die Erinnerungen anschlossen, die im neuronalen Netz der Zuhörenden aufgezeichnet waren. Darüber hinaus machten sie aber auch deshalb Eindruck, weil sie über die Amygdala zugleich emotionalisierend wirkten.

Im Vergleich dazu hat in den Wahlkampf-Reden von Adenauers jüngster Nachfolgerin im Kanzleramt, Angela Merkel, der doppelte Kern der Adenauer-Reden an Bedeutung verloren. Das gilt bereits für die Reden von Merkels Amtsvorgängern. Der Bezug zur deutschen Vergangenheit bleibt jedoch ein wichtiges und keineswegs aufgegebenes Element. Es wird immer dann eingebaut, wenn tagespolitische Ereignisse in den historischen Zusammenhang der Nachkriegspolitik gestellt werden. Das aber heißt, dass die Reden der Kanzlerin und der Kanzler bis heute Impuls-Reden mit hohem Erregungspotential geblieben sind. Im Falle von Merkel werden diese Reden durch eine Gestik der Bescheidenheit unterstrichen, die Adenauers sowohl rhetorischen als auch gestischen Vorgaben entsprechen.

Die Suche nach auffälligen Merkmalen der Reden, die amerikanische Präsidenten an ihr Volk halten, führt auch hier zur Charakterisierung als ImpulsReden. Bei Präsident Reagan waren sie - wie auch bei anderen Präsidenten - 
durch den Bezug auf Einschnitte der amerikanischen Geschichte gekennzeichnet. Vor allem wird auf die Zeit der Ablösung vom britischen Mutterland, auf die schwierigen Jahre der Herausbildung eines Föderalstaats und auf die Phase der Bewährung im Bürgerkrieg hingewiesen. Dies berührt im Langzeitgedächtnis der Bürgerinnen und Bürger aufbewahrte und sie zugleich emotionalisierende Ereignisse, von denen für jeweils aktuelle Herausforderungen Kraft, Mut und Zuversicht abgeleitet werden.

Dieselbe rhetorische Impulsgebung, unterstrichen durch Gestik und Mimik, bestimmte auch die Auftritte des Diktators Fidel Castro. Zwar verstieß er gegen das hirngerechte Prinzip eines auf Kürze und inhaltliche Beschränkung angelegten Rede-Designs. Auffällig sind jedoch Castros Bezüge zur heroischen Geschichte seines Landes und seiner persönlichen Mitwirkung an der Befreiung von kolonialer Abhängigkeit. Castro verwies sein Publikum auf die Vergangenheit und stellte diese ebenso glorreich dar, wie dies amerikanische Präsidenten tun, um bei ihren Bürgerinnen und Bürgern optimistische Stimmung zu verbreiten.

Nach demselben Muster, das Erinnerung/Wissen mobilisiert und dabei zugleich Emotionen weckt, sind die Reden konstruiert, die Parteiführerinnen und Parteiführer an Mitglieder sowie Funktionärinnen und Funktionäre, zugleich aber auch an ihre politischen Gegnerinnen und Gegner richten. Impulse dieser Art sandten beispielsweise August Bebel und Willi Brandt aus, die - in jeweils unterschiedlicher historischer Konstellation - auf heroische Zeiten ihrer Sozialdemokratischen Partei verweisen. Bebel bezog sich auf die Zeit der Bewährung im Kaiserreich, als dessen reaktionäre Kräfte mit Hilfe des Sozialistengesetzes seine Partei ausschalten wollten. Brandt schöpfte Hoffnung daraus, dass die Sozialdemokratie in der Zeit des Nationalsozialismus Widerstand leistete und sich nicht unterkriegen ließ.

Neurobiologisch betrachtet schaffen diese Redeelemente emotionalen Aufruhr im limbischen System. Er weckt Bereitschaft dafür, in aktuellen Situationen der Bewährung dieselben Kräfte zu mobilisieren, dieselben Opfer- und Ausdauer-Mentalitäten zu zeigen, die im kollektiven Gedächtnis aufbewahrt liegen und durch einschlägige Rede-Passagen aufgerufen werden können. Zur rhetorisch attraktiven Ausgestaltung von Impuls-Reden, d. h. zur Tonlage und ihrer gestisch-mimischen Begleitung, tritt somit der neuronal anknüpfungsfähige Redeinhalt.

Speziell muss das Gewicht der potentiellen Ausstrahlung betont werden, die den redenden Personen sowohl in als auch außerhalb von Hörsälen zukommt. Sie wurde im IV. Kapitel (IV.1./2.) charismatisch genannt. In neurobiologischer Sicht ist damit die sowohl gestisch-rhetorische als auch inhaltliche Ambiguität ihres Auftritts gemeint, die oben am Beispiel der Neuroästhetik illustriert wurde. Man denke an den „Impuls-Redner“ Hitler, den „Blut-und- 
Tränen-Redner" Churchill oder auch an der französischen Politik-Tradition (Pétain, de Gaulle, Mitterand), die ihre Zuhörenden in je besonderen Rahmenbedingungen elektrisierten.

Auch für das in der gesellschaftlichen Religionspraxis wichtige Format der Predigten gilt - wie schon für die politischen Programm-Reden -, dass charismatische Eigenschaften gefragt sind. Darüber hinaus gewinnen aber Predigten ihre neuronale Eingängigkeit daraus, dass sie thematisch eine große Erregungstiefe erreichen können. Geht es doch im Predigtformat in dem Sinne ans Eingemachte, dass besprochen wird, was die Einzelnen ebenso in ihrem Selbstverständnis wie in ihren Beziehungen zu den Mitmenschen und zudem in ihrem Verhältnis zur rätselhaften Gestalt eines allmächtigen Gottes betrifft. Dabei kommt fast immer gebotswidriges Versagen in diesen Beziehungen zur Sprache. In den Gehirnen der Beteiligten werden hierdurch konkurrierende Botenstoffe (u. a. Dopamin, Serotonin, Oxytozin) mobilisiert. Vor allem im limbischen System entsteht aufgrund der ungeklärten Frage Unruhe, ob Sünden und welche Sünden schlimm sind. Können sie - in der Familie, im Freundeskreis, am Arbeitsplatz oder auch beim Bordellbesuch begangen - wiedergutgemacht werden? Insoweit sind Predigten in der Tendenz immer Bußpredigten. Diese mittelalterliche Tradition war auffällig noch in den Auftritten präsent, die der fulminante katholische Pater Leppich in den 1950er Jahren hinlegte. Beispielsweise scheute er nicht davor zurück, Prostituierte und Freier auf der Hamburger Reeperbahn in dramatisierender Rede zur Umkehr zu mahnen. Wenngleich im Gestus mitfühlenden Verständnisses und geduldiger Nachsicht, bestimmt dieses Versagens-Thema, zumal der Verstoß gegen die herrschende Sexualmoral, auch manche Redepassagen, die der aus der katholischen Kirche gedrängte Eugen Drewermann an ein massenhaft versammeltes Publikum richtet. Die Eindringlichkeit solcher Reden entsteht aus der Verknüpfung des großen Versagens-Themas mit den vielen kleinen Beziehungsproblemen des Alltags, die das erworbene neuronale Potential in netzübergreifender Vielspurigkeit anspricht.

In Verbindung mit dem schockartigen Erlebnis, das der Tod - besonders der Verlust eines nahestehenden Menschen - bedeutet, gehen von Trauerreden Wirkungen aus, die in ihrer emotionalen Erregungstiefe von keinem anderen Thema erreicht werden. Dabei ist auch in ihnen der punktuelle Tiefgang mit Breitenwirkungen kombiniert. Die Redewendungen der Beschwichtigung des Todes, die den Grundtenor von Trauerreden bestimmen, schließen die Vielfalt der mitmenschlichen Probleme ein, die - wie erwähnt - auch vom Predigt-Format erfasst werden. Nach dem ,christlichen Schema“ (Ariès) wird in Trauerreden auf ein Jenseits verwiesen, das früher oder später zumindest die Chance bietet, all diejenigen wiederzutreffen, mit denen man im alltäglichen Leben zusammen war: Eltern, Geschwister und Verwandte, Freundinnen und Freunde, Arbeitskolleginnen und -kollegen. 
Nach dem ,säkularen Schema“ wird in Trauerreden vor allem auf das Lebenswerk der Toten, aber auch auf diejenigen Zurückbleibenden eingegangen, die an ihm Anteil hatten. Denen, die noch da sind, wird in säkular orientierten Trauerreden oft auch gesagt, dass von ihnen eine Fortsetzung des Werkes erwartet wird, das die Toten hinterlassen. Auf diese Weise werden alle in der Erinnerung aufbewahrten und oft aufwühlenden Erlebnisse angesprochen, die diejenigen, die gestorben sind, mit denjenigen teilen, die noch mit einem kürzeren oder längeren Weiterleben rechnen können.

\section{Instruktions-Reden außerhalb von Hörsälen - hoher Lernertrag im Ausnahmefall}

Es ist für Vorlesungen des Typs der frontalen Instruktions-Rede gezeigt worden, dass zwischen dem Anspruch, in großem Umfang Erinnerung/Wissen zu vermitteln, und dem, was „hängen bleibt“, ein großes Missverhältnis besteht (Kap. IV.3.). Für Reden außerhalb von Hörsälen gilt dies offensichtlich für die Routine-Veranstaltungen, die von sogenannten Ostblock-Politikern absolviert wurden. Man denke an die hölzerne Monotonie, in der Walter Ulbricht oder Erich Honecker die Erfüllung von DDR-Planziffern meldeten und in ebenso langatmigen Einzelheiten eine erfolgreiche „,sozialistischen Zukunft“ voraussagten. Warum sollte sich der geringe Instruktionsertrag ähnlich gestrickter frontaler Reden, die außerhalb von Hörsälen gehalten werden, nicht auch in Konstellationen des gesellschaftlichen Alltags zeigen? Und woran liegt es im Ausnahmefall, dass auch Instruktions-Reden ertragreich sein können?

Wer Gruppen beobachtet, die eine Stadtbesichtigung machen, oder wer selbst an solchen Besichtigungen teilgenommen hat, wird um folgende Feststellungen kaum herumkommen: Je länger die Besichtigungen dauern, desto anstrengender und langweiliger werden sie. Je mehr Informationen - vor allem Daten und Zahlen - ausgespuckt werden, desto geringer ist am Schluss das Lernergebnis. Der Ausnahmefall kann darin bestehen, dass die besichtigte Stadt vor allem in ihren visuellen Angeboten (Architektur, Kunstschätze und -gegenstände) unter Gesichtspunkten der Belohnung (Orbitofrontaler Cortex) von besonders attraktiver Schönheit ist. Bis zu einem gewissen Grade ist der Ausnahmefall auch arrangierbar, wenn Besichtigungen etwa bei Nacht und durch emotionalisierende Nachtwächter-Folklore (plus personellem Faktor) dekoriert werden. Eingängig sind sie aber vor allem dann, wenn sich das besichtigende Publikum vorbereitet hat, so dass die vielfältigen Informationen auf „fruchtbaren Boden fallen“ und „,aufgehen“ können.

In der Konstellation von Reisegruppen, die durch eine Ausgrabungsstätte sagen wir, der kretisch-minoischen Kultur - geführt werden, kann es eine be- 
sonders „farbige Persönlichkeit“ sein, von der die Gruppenmitglieder aufgestört und in ihrer Merkfähigkeit herausgefordert werden. Ein im Ausnahmefall erreichbarer Instruktionsertrag ist aber vor allem davon abhängig, dass sich die Gruppenmitglieder oder auch nur einzelne Teilnehmende auf die Kultur, der sie begegnen, vorbereitet haben. Auf diese Weise die ihnen vorgetragenen Einzelheiten im Langzeitgedächtnis Anknüpfungspunkte: Vorhandene Kenntnisse zum Verlauf der Geschichte der Minoer werden präzisiert. Auffällige Merkmale der Palastanlagen - ihre Architektur und Raumverteilung lassen sich im Detail betrachten, noch erhaltene und restaurierte Fresken bewundern. Im Ergebnis bleiben Erinnerungs- und Wissensbestände zurück, in denen das von der Reiseleitung beigesteuerte Neue sich mit den Kenntnissen verbindet, die Mitglieder der Reisegruppe mitgebracht haben.

\section{Exkurs 3}

\section{Die sogenannten Spiegelneuronen und die verkürzte Vorstellung von hirngerechtem Lernen}

Die hier vorgestellten Analysen und Überlegungen zielen auf eine hirngerechte Bestimmung und Ausgestaltung von ertragreichen Lernformen. Dem steht ein Ansatz entgegen, in dem die neuronale Abstützung von Interaktionen - speziell auch von Lernprozessen - unangemessen reduzierend und verkürzend betrachtet wird. Diesem Ansatz, der mit suggestiver Spiegel-Metaphorik arbeitet, muss hier widersprochen werden.

In seiner Politeia nimmt Plato für die Philosophie in Anspruch, die inneren Strukturen des Seins zu erfassen. Demgegenüber kritisiert er die Kunst - insbesondere die Malerei - dafür, dass sie auf äußeren Schein gerichtet sei und die Wahrheit verfehle. Maler sieht er als lächerliche Träger von Spiegeln, mit deren Hilfe sie das Äußere der Dinge einfach abkupferten (Welsch 2011: 23ff).

Indessen kann sich selbst Plato der trügerischen Wirksamkeit des Spiegelbilds nicht entziehen (Welsch 2011: 27f.). Sein fortdauernder Zauber, den u. a. Leonardo da Vinci zum künstlerischen Ideal gemacht hat (Welsch 2011: 29f.), ist unverkennbar, wenn sich Gesichter, oder auch künstliche Objekte im Wasser spiegeln und auf Beobachtende in hohem Maße emotionalisierend wirken. So ist es nicht überraschend, dass eine Forschungsgruppe um Giacomo Rizzolatti von der Magie des Spiegelbilds seit sie zunächst in den motorischen Arealen von Affen und dann auch bei anderen Tieren spezielle Neuronengruppen entdeckte und diese Spiegelneuronen nannte (u. a. Rizzolatti/Sinigaglia 2008). 
Gemeint war und ist im Ergebnis nachfolgender Forschungen das Folgende: Die Neuronen des Spiegel-Typs haben eine doppelte Funktion. Sie werden sowohl bei eigenen Bewegungen von Probanden als auch dann mobilisiert, wenn die Bewegungen von Partnerinnen und Partnern bei der Kommunikation beobachtet werden. Es gilt das Prinzip der sich überschneidenden neuronalen Repräsentationen. Für das, was mich selbst betrifft, beispielsweise mein Essen einer Banane oder mein Greifen nach einer Tasse, sind dieselben neuronalen Areale zuständig, die aktiviert werden, wenn andere ihre Banane essen und nach ihrer Tasse greifen. Als wichtige Beobachtung kommt hinzu, dass die jeweils zuständigen Neuronen und Gruppen von Neuronen nicht nur eine Vorstellung von je eigenen Bewegungen liefern, sondern zugleich ein Verständnis für die Bedeutung vermitteln, die Bewegungen für andere haben (Rizzolatti/ Sinaglia: 14). Im Ganzen wird eine Parallelität zwischen neuronal gestützten Eigenbewegungen und beobachteten Fremdbewegungen belegt, die durch die Metapher des Spiegelbilds nicht angemessen - wenngleich suggestiv wirksam - erfasst wird.

Die Doppelfunktion des neuronalen Spiegeltyps passt zu Antonio Damasios Hypothese, nach der Gehirne die Fähigkeit haben, sowohl eigene Körperzustände als auch diejenigen von anderen Personen zu simulieren. Daher sind für ihn ,die sogenannten Spiegelneuronen ... die höchste Form eines Als-ob-Apparats des Körpers" (Damasio 2010: 115). Das aber heißt, dass die Gehirne nicht über alle Areale hinweg nach dem Prinzip doppelter neuronaler Funktionalität arbeiten. Auch die inzwischen schon weit verzweigte Empathie-Forschung, zu der die Forschungsergebnisse gehören, die auf den Spuren von Rizzolatti u. a. erzielt wurden, belegt dies. Die Aktivität der sogenannten Spiegelneuronen ist auf zielorientiertes Handeln beschränkt, das von den prämotorischen Abschnitten des Stirnhirns getragen wird (Watson/Greenberg, in: Decety/Ickes 2011: 127).

Demgegenüber ist die neuronale Abstützung empathischer Fähigkeiten, sind sowohl kognitive als auch affektive Dispositionen, die das Hineindenken (sog. theory of mind) und das Hineinfühlen in die Lage anderer Personen ermöglichen, im neuronalen Netzwerk weit verstreut. Nach der zusammenfassenden Darstellung von Watson und Greenberg umfassen sie insbesondere diejenigen Abschnitte des Gehirns, die für Emotionen zuständig sind und im Umkreis des limbischen Systems liegen. Einbezogen sind der Anteriore Cinguläre Cortex (ACC), die Insula, der Thalamus, somatosensorische Cortices und auch der rechte inferiore Scheitellappen (ebda.).

Nun lässt sich aber in der Anwendung der Ergebnisse der Empathie-Forschung eine Tendenz beobachten, Interaktionen unterschiedlichster Art aus der Perspektive der sogenannten Spiegelneuronen zu betrachten. Indem aus ihrer speziellen Funktionsweise allgemeine Schlüsse auf das breite Spektrum 
empathischer Fähigkeiten sowohl von tierischen als auch von menschlichen Primaten gezogen werden, wird die nur sektorale Bedeutung des neuronalen Spiegeltyps überspielt.

Dies gilt speziell für Anwendungen auf die hier interessierende Pädagogik. Besonders - auch für die Hochschuldidaktik - ist hier der Freiburger Neurobiologe und Psychotherapeut Joachim Bauer. Er beschränkt die Funktionsweise der sogenannten Spiegelneuronen einerseits korrekt auf das Feld motorischer Aktivitäten (Bauer 2006: bes. 40f.). Andererseits erweitert er jedoch ihren Zuständigkeitsbereich. Sie sind für ihn das Kernstück der neuronalen Basis, auf der das gesamte Spektrum der mitmenschlichen Beziehungen und damit auch das Verhältnis der Lehrpersonen zu ihren Schülerinnen und Schülern beruht. Wie problematisch es ist, dieses zentral auf die Spiegelneuronen zu beziehen, zeigt sich an einem Beispiel, das Bauer zur allgemeinen Illustration auswählt. Es betrifft deutlich den Bereich motorischer Aktivitäten, wenn eine Situation geschildert wird, in der ,zwei Waldarbeiter mit einer großen Handsäge arbeiten, die sich von beiden Seiten fassen und wechselseitig hin- und herführen lässt"“ (Bauer 2009: 111).

Nach dem Muster dieser motorischen Beziehung wird die Kommunikation zwischen Lehrpersonen und Lernenden von Bauer als Spiegelverhältnis beschrieben: Durch ihr Auftreten erzeugen Lehrpersonen ein Spiegelbild, das vom Spiegelsystem der Schülerinnen und Schüler verarbeitet wird. Umgekehrt werden auch sie aktiv und versorgen ihre Lehrpersonen mit ihrem Spiegelbild, das von diesen wahrgenommen wird (ebda.: 113). Da es aber in diesen Beziehungen offensichtlich um mehr als das wechselseitige Verhältnis zwischen sägenden Waldarbeitern geht, können empathische Beziehungen auf solche Weise nicht angemessen erfasst werden. Im Besonderen verstellt eine einseitige Konzentration auf die sogenannten Spiegelneuronen den Blick dafür, dass ertragreiche Lernprozesse - vermittelt über Kurz- und Langzeitgedächtnis einer breiten neuronalen Abstützung bedürfen. Dies wurde in Kapitel II.2 gezeigt. Dabei stand das Problem geeigneter Lernformen, das bei Joachim Bauer u. a. fehlt, im Zentrum des Interesses. ${ }^{67}$

Unter dem offensichtlichen von Bauer schildern die Hochschuldidaktiker Andreas Böss-Ostendorf und Holger Senft, mit Blick auf zielgerichtete Aktionen, die doppelte Wirkungsweise von sogenannten Spiegelneuronen. In Übereinstimmung mit Bauer erweitern sie deren Zuständigkeitsbereich und übergehen auf diese Weise die breite neuronale Abstützung empathischer Beziehungen. Dies zeigt die folgende generalisierende Behauptung: „Der Lehrer

67 Vgl. zur irreführenden Reduzierung des Empathiesystems auf das „SpiegelneuronenSystem“ Roth/Ryba 2016: 141. 
(gemeint ist der Hochschullehrer; d. Verf.), der im Seminar anwesend ist, ist eben auch in Hinsicht auf die hirnphysiologischen Prozesse immer ein lebendiges Beispiel dafür, wie in einer Fachdisziplin gedacht und gearbeitet wird.“ (Böss-Ostendorf/Senft 2018: 97) Auch diese Autoren sparen dann die Probleme hirngerechter Lernformen aus. Können Lehrpersonen „im Seminar“ (wie ausgestaltet?) oder im Vorlesungsbetrieb zum „Denken“ und „Arbeiten“ ihres Faches anleiten? In neurobiologischer Perspektive können solche Fragen nicht allein auf die sogenannten Spiegelneuronen bezogen werden, da diese nur für zielgerichtet motorisches Handeln zuständig sind. 


\section{Literaturverzeichnis}

\section{Theorie und Methodologie der modernen Gesellschaft}

Buschak, Willy, 1985: Von Menschen, die wie Menschen leben wollten. Geschichte der Gewerkschaft Nahrung-Genuss-Gaststätten und ihrer Vorläufer, Köln.

Dreyfus, Hubert L./Paul Rabinow, 1987: Michel Foucault. Jenseits von Strukturalismus und Hermeneutik, Frankfurt/M.

Edelman, Murray, 1976: Politik als Ritual. Die symbolische Funktion staatlicher Institutionen und politischen Handelns, Frankfurt-New York.

Eribon, Didier, 1993: Michel Foucault. Eine Biographie, Frankfurt/M.

Foucault, Michel, 1976: Die Geburt der Klinik. Eine Archäologie des ärztlichen Blicks, Frankfurt/M.-Berlin-Wien.

Foucault, Michel, 1977: Überwachen und Strafen, Frankfurt/M.

Galtung, Johan, 1994: Menschenrechte - anders gesehen, Frankfurt/M.

Geißler, Rainer, 1985: Technikfeindlich und leistungsscheu? Zum Einstellungswandel der Jugend, in: Aus Politik und Zeitgeschichte, B 38/85: 27-37.

Habermas, Jürgen, 1962: Strukturwandel der Öffentlichkeit, Neuwied-Berlin.

Kirchheimer, Otto, 1965: Der Wandel des westdeutschen Parteiensystems, in:

Politische Vierteljahresschrift, 6. Jg.: 24-41.

Loewenstein, Karl 1959: Verfassungslehre, Tübingen.

Maslow, Abraham, 1954: Motivation and Personality, New York u. a.

Nassehi, Armin, 2009: Der soziologische Diskurs der Moderne, Frankfurt/M.

Ringer, Fritz K., 1983: Die Gelehrten. Der Niedergang der deutschen Mandarine 1890-1933, Stuttgart.

Schirrmacher, Frank, 2009: Payback. Warum wir im Informationszeitalter gezwungen sind zu tun, was wir nicht wollen, und wie wir die Kontrolle über unser Denken zurückgewinnen, München.

Searle, John R., 1997: Die Konstruktion der gesellschaftlichen Wirklichkeit, Reinbek.

Seel, Wolfgang, 1985: Bildungs-Egoismus. Alle wollen mehr, Osnabrück.

Shell Deutschland Holding (Hrsg.), 2010: Jugend 2010. Eine pragmatische Generation behauptet sich, Frankfurt/M.

Vilmar, Fritz 1973: Strategien der Demokratisierung, Bd. I: Theorie der Praxis, Darmstadt-Neuwied.

Welsch, Wolfgang 2011: Immer nur Mensch? Entwürfe zu einer anderen Anthropologie, Berlin. 


\section{Prinzipien der Neurobiologie}

Bauer, Joachim, 2007: Prinzip Menschlichkeit. Warum wir von Natur aus kooperieren. Hamburg.

Birbaumer, Niels/Schmidt Robert, 2010: Heidelberg.

Bundesministerium für Forschung und Technologie (BMFT), 2007: Auf den Anfang kommt es an: Perspektiven für eine Neuorientierung frühkindlicher Bildung, Bonn-Berlin.

Damasio, Antonio R., 2005: Der Spinoza-Effekt. Wie Gefühle unser Leben bestimmen, Berlin.

Damasio, Antonio R., 2011: Selbst ist der Mensch. Körper, Geist und die Entstehung des menschlichen Bewusstseins, München.

Decety, Jean/Ickes, William (Ed.), 2011: The Social Neuroscience of Empathy, Cambridge/Massachusetts u. a.

Fuchs, Thomas, 2007: Das Gehirn - ein Beziehungsorgan. Eine phänomenologisch-ökologische Untersuchung, Stuttgart.

Gallese, Vittorio/Giovanni Buccino, 2011: Wir und die anderen. Von den Spiegelneuronen zum Mitgefühl, in: Spitzer, Manfred/Wulf Bertram (Hrsg.), Hirnforschung für Neu(ro)gierige. Braintertainment 2.0, Stuttgart.

Kandel, Eric R. u. a., 2000: Principles of Neural Science, Mc Graw-Hill.

Kandel, Eric R., 2006: Psychiatrie, Psychoanalyse und die neue Biologie des Geistes, Frankfurt/M.

Koch, Christoph, 2005: Bewusstsein. Ein neurobiologisches Rätsel, Berlin Heidelberg.

LeDoux, Joseph, 1998: Das Netz der Gefühle. Wie Emotionen entstehen, München-Wien.

Pinker, Steven, 2003: Das unbeschriebene Blatt. Die moderne Leugnung der menschlichen Natur, Berlin.

Pöppel, Ernst, 2006: Der Rahmen. Ein Blick des Gehirns auf unser Ich, München.

Rizzolatti, Giacomo/Corrado, Sinigaglia, 2008: Empathie und Spiegelneurone. Die biologische Basis des Mitgefühls, Frankfurt/M.

Rösler, Frank, 2011: Psychophysiologie der Kognition. Eine Einführung in die Kognitive Neurowissenschaft, Heidelberg.

Roth, Gerhard, 2003: Fühlen, Denken, Handeln. Wie das Gehirn unser Verhalten steuert, Frankfurt/M.

Roth, Gerhard/Ryba, Alica 2016: Coaching, Beratung und Gehirn. Neurobiologische Grundlagen wirksamer Veränderungskonzepte, Stuttgart.

Searle, John R., 2004: Freiheit und Neurobiologie, Frankfurt/M.

Singer, Tania, 2006: The neural basis and ontogeny of empathy and mind reading: Review of literature and implications for future research, in: Neuroscience and Biobehavioral Reviews, 30: 855-863. 
Tomasello, Michael, 2010: Warum wir kooperieren, Berlin.

Verplaetse, Jan, 2011: Der moralische Instinkt. Über den natürlichen Ursprung unserer Moral, Göttingen.

Woolfolk, Anita, 2008: Pädagogische Psychologie, München.

Wuketits, Franz M., 1993: Evolutionäre Ethik und ihre Kritiker. Versuch einer Metakritik, in: Lütterfelds, Wilhelm/Thomas Mohrs (Hrsg.), Evolutionäre Ethik zwischen Naturalismus und Idealismus. Beiträge zu einer modernen Theorie der Moral, Darmstadt.

\section{Theorie und Empirie des Lernens und seine neurobiologischen Grundlagen}

Barr, Robert B./John Tagg, 1995: From teaching to learning - A new paradigm for undergraduate education, in: Change, Vol. 27, No. 6 (1995).

Bauer, Joachim, 2006: Warum ich fühle, was du fühlst. Intuitive Kommunikation und das Geheimnis der Spiegelneurone, München (4.

Bauer, Joachim, 2009: Erziehung als Spiegelung. Die pädagogische Beziehung aus dem Blickwinkel der Hirnforschung, in: Herrmann, Ulrich (Hrsg.), Neurodidaktik. Grundlagen und Vorschläge für gehirngerechtes Lernen, Weinheim Basel (2.

Böss-Ostendorf, Andreas/Senft, Holger 2018: Einführung in die Hochschul-Lehre. Der Didaktik-Coach, Opladen \& Farmington Hills (3.

Dante Costa Santos/Strasser, Patrick, 2014: Ich, Dante: Aus armen Verhältnissen in Brasilien zum FC Bayern München, München.

Dehaene, Stanislas, 2014: Denken. Wie das Gehirn Bewusstsein schafft, München.

Dehaene, Stanislas, 2010: Lesen. Die größte der Menschheit und was dabei in unseren Köpfen passiert, München.

Dormehl, Luke, 2016: Thinking Machines. The inside story of Intelligence and our race to build the future, London.

Carey, Benedict, 2015: Neues Lernen. Warum Faulheit und Ablenkung dabei helfen, Hamburg (2.

Howard-Jones, Paul u. a., 2011: Toward a science of Learning Games, in: Mind, Brain, and Education, Volume 5, Issue 1.

Kiefer, Markus u. a., 2008: Die Rolle der motorischen Interaktion beim Erwerb

Wissens. Eine Trainingsstunde mit künstlichen Objekten, in: Zeitschrift für Pädagogische Psychologie (2008), 22: 47-58.

Kiefer Markus, „Arbeitsgruppe Struktur des semantischen Gedächtnisses“, unter Einschluss der Mitarbeiterliste und des Auftraggebers; online verfügbar unter: htpps://www.uni-ulm.de/dfg-kiefer.html (zuletzt geprüft. 10.08.2018). 
Korte, Martin, 2017: Wir sind Gedächtnis. Wie unsere Erinnerungen bestimmen, wer wir sind, München.

Macedonia, Manuela, 2017: Ein Billie für alle Fälle, in: Gehirn \& Geist, Dossier 4/2017, S. 20-25.

Miller, Earl K./Buschman, Timothy J., 2013: Brain Rhythms For Cognition and Consciousness, in: Neurosciences and the Human Person: New Perspectives on Human Activities,

Academy of Sciences, Scripta Varia 121, Vatican City.

Pöppel, Ernst, 2008: Zum Entscheiden geboren. Hirnforschung für Manager, München.

Pöppel, Ernst, 2010: Alpha Forum (WDR), Gespräch mit Iska Schreglmann v. 29.04.2010.

Roth, Gerhard, 2011: Bildung braucht Persönlichkeit. Wie Lernen gelingt, Stuttgart.

Spitzer, Manfred, 2007: Lernen. Gehirnforschung und die Schule des Lebens, Berlin-Heidelberg.

Spitzer, Manfred, 2015: Cyberkrank! Wie das digitalisierte Leben unsere Gesundheit ruiniert, München.

Vogeley, Kai/Newen, Albert, 2006: Ich denke was, was du nicht denkst, in: Könneker, Carsten (Hrsg.), Wer erklärt den Menschen? Hirnforscher, Psychologen und Philosophen im Dialog, Frankfurt/M.

Metzinger, Thomas, 2010: Der EGO-Tunnel. Eine neue Philosophie des Selbst: Von der Hirnforschung zur Bewusstseinsethik, Berlin.

Reimann, Bruno W., 1978: Hochschulreform - Illusion und Pleite? Bonn.

Urchs, Max, 2002: Maschine, Körper, Geist. Eine Einführung in die Kognitionswissenschaft, Frankfurt/M.

Zeki, Semir, 2010: Glanz und Elend des Gehirns. Neurobiologie im Spiegel von Kunst, Musik und Literatur, München-Basel.

Ziegler, Jean, Interview, in: Zeit-Magazin, 20.12.2017.

\section{Historische Entwicklung der deutschen Universitäten}

Bartz, Olaf, 2007: Der Wissenschaftsrat. Entwicklungslinien der Wissenschaftspolitik in der Bundesrepublik Deutschland 1957-2007, Stuttgart.

Baumgarten Eduard, 1963: Gedanken zur künftigen Hochschule (1960), in: Ders., Zustand und Zukunft der deutschen Universität, Tübingen.

Bisky, Jens, 2011: Wie die schriftliche Hausarbeit an die preußischen Universitäten kam, in: Süddeutsche Zeitung, 22.02.2011.

Braungart, G. u. a., 1981: Studium 80 - Hochschule in der Diskussion, Ein Lese- und Arbeits-Buch, Stuttgart. 
Ellwein, Thomas, 1997: Die deutsche Universität. Vom Mittelalter bis zur Gegenwart, Wiesbaden.

Friedeburg, Ludwig von u. a., 1968: Freie Universität und politisches Potential der Studenten. Über die Entwicklung des Berliner Modells und den Anfang der Studentenbewegung in Deutschland, Neuwied-Berlin.

Humboldt, Wilhelm von, 1956: Die Idee der deutschen Universität, Darmstadt. Jens, Walter/Jens, Inge, 2010: Eine deutsche Universität. 500 Jahre Tübinger Gelehrtenrepublik, Reinbek bei Hamburg.

Morkel, Arnd, 2000: Die Universität muss sich wehren. Ein Plädoyer für ihre Erneuerung, Darmstadt.

Mosse, Werner, 1988: Adel und Bürgertum im Europa des 19. Jahrhunderts. Eine vergleichende Betrachtung, in: Jürgen Kocka (Hrsg.), Bürgertum im 19. Jahrhundert. Deutschland im europäischen Vergleich, Bd. 2, München.

Straub, Eberhard, 2007: Vom Knattermimen zum Talkmaster, in: Stifterverband der deutschen Industrie, W\&W 2007/4.

Straub, Eberhard, 2008: Vom „Glück“ der Vorlesungen (2007), in: Ders., Deutschland Deine Bildung! Essays zur Idee und Geschichte, Berlin.

\section{Studentische Erfahrungen an Hochschulen und Hochschuldidaktik}

Apel, Hans Jürgen, 1998: „Freier Vortrag vor mitdenkenden Köpfen ...“. Geschichte und Zukunft der Vorlesung, in: Forschung \& Lehre, 2/98, S. 60-63. Apel, Hans Jürgen, 1999: Die Vorlesung. Einführung in eine akademische Lehrform, Köln-Weimar-Wien.

Bargel, Tino u. a., 1989: Studienerfahrungen und studentische Orientierungen. Drei Erhebungen zur Studiensituation an Universitäten und Fachhochschulen in den Wintersemestern 1983/83, 1984/85 und 1986/87 (Kurzfassung), Bonn.

Bargel, Tino u. a., 2009: Bachelor-Studierende. Erfahrungen in Studium und Lehre. Eine Zwischenbilanz, Berlin.

Behrendt, Brigitte, 2005: „The Shift from Teaching to Learning“ - mehr als eine ,Redewendung': Relevanz - Forschungshintergrund - Umsetzung. Johannes Wildt zum 60. Geburtstag, Bielefeld.

Borgwardt, Angela/Felmet, Anne, 2018: Spannung im Hörsaal. Wie gelingt gute Lehre an Hochschulen? Schriftenreihe Hochschulpolitik, Berlin.

Bourdieu, Pierre, 1984: homo academicus, Paris.

Bruch, Rüdiger vom, 2002: Der Vorleser? Historische Steifzüge zur Vorlesung, in: Forschung \&Lehre, 10/2002: 524-526. 
Dempf, Peter, 1988: Das hundertfache Nein. Tagebuch eines frisch ausgebildeten Gymnasiallehrers, der ein Unterkommen sucht, in: Süddeutsche Zeitung, 27./28.8.1988.

Dräger, Jörg/Müller-Eiselt, Ralph, 2015: Die digitale Bildungsrevolution. Der radikale Wandel des Lernens und wie wir ihn gestalten können, München.

Fernandez,Villanneva, 2009: Interaktive Vorlesungen: Wie bitte? Zur Rolle der Lehrenden in Vorlesungen aus der Sicht renommierter Hochschullehrender, in: Lévy-Todter, Magdalène/Meer, Dorothee (Hrsg.): Hochschulkommunikation in der Diskussion, Frankfurt/M.

Florin, Christiane, 2014: Warum unsere Studenten so angepasst sind, Reinbek bei Hamburg.

Foucault, Michel, 1999: Les anormaux. Cours au Collège de France (19741975), Paris.

Fuchs, Peter, 2011: Jedes Original ist ein listiges Plagiat und umgekehrt - Zur Funktion des Plagiierens in der modernen Gesellschaft, in: Rommel, Thomas (Hrsg.): Plagiate - Gefahr für die Wissenschaft? Eine internationale Bestandsaufnahme, Berlin.

Gleich, Johann Michael u. a., 1982: Studenten und Hochschullehrer. Eine empirische Untersuchung an den baden-württembergischen Universitäten, Villingen-Schwenningen.

Grühn, Dieter u. a. (Hrsg.), 1992: Evaluation von Lehrveranstaltungen. Überfrachtung eines sinnvollen Instrumentes?, Berlin.

Hamers, Joachim, 1978: Die Universitätsausbildung von Sozialwissenschaftlern, Frankfurt-New York.

Handke, Jürgen, 2014: Patient Hochschule. Vorschläge für eine zeitgemäße Lehre im 21. Jahrhundert, Marburg.

Handke, Jürgen, 2015: Handbuch Hochschule digital. Leitfaden für eine moderne und mediengerechte Lehre, Marburg.

Handke, Jürgen, 2016: Die Wirksamkeit der Präsenzphase im Inverted Classroom, In: Großkurth, Eva Marie/Jürgen Handke (Hrsg.): Inverted Classroom and Beyond. Lehren und Lernen im 21. Jahrhundert, 4. ICM-Fachtagung an der Philipps-Universität Marburg, Marburg.

Hattie, John, 2014: Lernen sichtbar machen, überarb. deutschsprachige Ausgabe von „Visible Learning“ (engl. Orig. 2009), besorgt von Wolfgang Beywl und Klaus Zierer, 2. korrig.

Hilbrich, Romy u. a. 2014: Qualität durch Differenzierung. Lehrpersonen, Lehrqualität und das Verhältnis von Lehre und Forschung, in: Beiträge zur Hochschulforschung, 36. Jg., 1/2014.

Hochschulforum Digitalisierung 2016: The Digital Turn. Hochschulbildung im digitalen Zeitalter, Edition Stifterverband - Verwaltungsgesellschaft für W 
Hennis, Wilhelm, 1969: Gespräch mit Gangolf Hübinger, in: Neue Politische Literatur (H. 3): 365-379.

Huber, Ludwig/Hellmer, Julia/Schneider, Friederike (Hrsg.), 2009: Forschendes Lernen im Studium. Aktuelle Konzepte und Erfahrungen, Bielefeld.

Klee, Bettina, 1989: Auf der Suche nach der verlorenen Hausarbeit. Eine Beschwerde, in: Koch, Angelika, 1993: „O Gott, noch ein Student, was will der jetzt von mir?" (Auszug aus einer Diplomarbeit 1993) zit. nach: Frankfurter Rundschau, 30.12.1993.

Krüger, Hans-Joachim u. a., 1986: Studium und Krise. Eine empirische Untersuchung über studentische Belastungen und Probleme, Frankfurt/M.

Loviscach, Jörn, 2016: Vortrag auf „futur iii“, HS Offenburg, 22.10.2016“ (,Bildung steht nicht nur im Modulhandbuch - von den Gefahren einer naiven Digitalisierung"), verfügbar unter: http:/www.j3L7h.de/videos.html (zuletzt geprüft: 10.08.2018).

Loviscach, Jörn, 2016: Digitalisierung der Hochschulausbildung zwischen Graswurzel und Business, 16.09.2016; Text online verfügbar unter: Digitalisierung der Hochschulausbildung zwischen Graswurzel und Business (zuletzt geprüft: 10.08.2018).

Loviscach, Jörn, 2018: Videos plus Quizze plus Präsenzlehre, 27.02.2018, online verfügbar unter: https://youtube/uk_GC2chdjw (zuletzt geprüft: 10.08.2018).

Meinel, Christoph 2017: Hochschulforum Digitalisierung („Eine Vision für die Zukunft digitaler Bildung“", 25.04.2017); online verfügbar unter: https://hochschulforumdigitalisierung de/de/blog/christoph-meinel (zuletzt geprüft: 10.08.2018)

Metz-Göckel, Siegrid, 1975: Theorie und Praxis der Hochschuldidaktik, Frankfurt/M.-New York.

Neues Handbuch Hochschullehre, 2006: Berlin.

Brigitta K., 2015: Lehren an Hochschulen: eine Hochschuldidaktik für den Aufbau von Wissen und Kompetenzen, Bern (2.

Pöppel, Ernst, 2010: „Von der letzten Vorlesung zum interkulturellen Dialog“, in: Ders./Beatrice Wagner, Je älter desto besser. Überraschende Erkenntnisse aus der Hirnforschung, München.

Rumpf, Horst, 1978: Alltägliche Hochschule. Über die szenisch vermittelte Lähmung von Subjekten, in: Klaus Horn (Hrsg.), Kritik der Hochschuldidaktik, Frankfurt/M.

Stroebe, Wolfgang, 2017: Große Vorsicht geboten. Was evaluieren Studierende, wenn sie Lehrveranstaltungen evaluieren? in: Forschung \& Lehre 2/17. Thieme, Werner, 1982: Hochschuldidaktik als Studienreform, in: Horst Albert Glaser (Hrsg.), Hochschulreform - und was nun?, Frankfurt/M. u. a.

Wagner, Wolf, 2010: Tatort Universität. Vom Versagen deutscher Hochschulen und ihrer Rettung, Stuttgart. 
Weber, Stefan, 2011: Das akademische Textplagiat in Österreich - Zwischen Rechtsprechung und Lehrbuchvorgaben einerseits und gelebter wissenschaftlicher Praxis andererseits, in: Rommel, Thomas (Hrsg.): Plagiate Gefahr für die Wissenschaft? Eine internationale Bestandsaufnahme, Berlin. Welbers, Ulrich u .a. (Hrsg.), 2005: The Shift from Teaching to Learning, Bielefeld.

Wieman, Carl: hochschuldidaktischer Zugriff online verfügbar unter: https://www. aaas.org/news/nobel/laureate-carl-wieman-effective-teaching-should-create-students-who-like-scientists (8 june 2012).

Winteler, Adi, 2011: Professionell lehren und lernen. Ein Praxisbuch. Unter Mitarbeit von Claudia Geyer und Gerhard Lehrberger, Darmstadt (4.

Wolf, Maryanne, 2009: Das lesende Gehirn. Wie der Mensch zum Lesen kam - und was es in unseren Köpfen bewirkt, Heidelberg.

Zierer, Klaus, 2017: Lernen 4.0. Pädagogik vor Technik, Möglichkeiten und Grenzen einer Digitalisierung im Bildungsbereich, Hohengehren. 


\section{Personenregister}

Adenauer, Konrad 135

Baars, B.J. 82

Bandura, Albert 48

Bargel, Tino 63f., 147

Barr, Robert B. 102, 145

Bartz, 27, 102, 146

Bauer, Joachim 42, 141, $144 f$.

Baumgarten, Eduard 86

Bebel, August 136

Becker, C.H. 61

Bender, Christiane 15

Birbaumer, Niels 38, 42f., 59, 144

Boess-Ostendorf, Andreas 17, 141, 145

Bonnet, Martin 117

Bourdieu, Pierre 87

Brandt, Willy 101136

Brecht, Bert 9

Brentano, Lujo 75

Castro, Fidel 136

Churchill, Winston 136

Dahrendorf, Ralf 27

Damasio, Antonio 32, 39, 43, 49, $68,140,144$

De Gaulle, Charles 137

Dehaene, Stanislas 16, 34, 96, 145

Deutsch, Karl 129

Dohm, Hedwig 53

Dräger, Jörg 106-108, 147

Drewermann, Eugen 137

Dürig, Günter 78

Edelman, Murray 45, 59f., 143

Elber, Giovane 95

Ellwein, Thomas 24, 28, 147
Eribon, Didier 47, 143

Eschenburg, Theodor 42

Fichte, Johann Gottlieb 24, 72

Flitner, Andreas 18

Florin, Christiane 51, 148

Foucault, Michel 31, 46, 55, 76, 78f., 88, 147

Fraenkel, Ernst 42

Freud, Sigmund 34

Friedeburg, Ludwig von 76, 146

Fuchs, Thomas 68, 144

Gadamer, Hans-Georg 40

Galtung, Johan 40, 143

Grottian, Peter 64

Guttenberg, Karl Theodor Freiherr von und zu $65,67 f$.

Habermas, Jürgen 45, 47, 90, 143

Häberle, Peter 65, 68

Handke, Jürgen 101, 109, 118, 121 , 148

Hatt, Hanns 33

Hattie, John 19f., 101, 107, 148

Hegel, Georg Wilhelm Friedrich 24, 72

Heidegger, Martin 75

Hennis, Wilhelm 14, 149

Herbart, Johann Friedrich 18

Herzfeld, Hans 74

Hitler, Adolf 137

Howard-Jones, Paul 45, 145

Humboldt, Wilhelm von 25, 53, 116, 120f., 133, 147

Jaspers, Karl $75 f$.

Jens, Inge 147

Jens, Walter 25f., 147 
Kandel, Eric 32, 42, 144

Kiefer, Markus 85, 145

Klafki, Wolfgang 18

Kluge, Alexander 73

Koch, Angelika 57

Koch, Christoph 32, 144

Korte, Martin 100, 146

LeDoux, Joseph 39, 144

Litt, Theodor 18

Löwenstein, Karl 26, 101, 143

Loviscach, Jörn 14, 53, 101, 115f., $117,122,149$

Maslow, Abraham 40, 143

Mead, George Herbert 31

Meinel, Christoph 115, 149

Merkel, Angela 135

Metzinger, Thomas 77, 146

Michelangelo, Buonarotti 134

Mitterand, Francois 137

Morkel, Arnd 76, 146

Mosse, Werner 24, 147

Nassehi, Armin 31, 143

Orwell, George 10

Pestalozzi, Johann Heinrich 18, 95

Pétain, Philippe 137

Picht, Georg 27

Pinker, Stephen 16, 40, 68, 144

Plato 139

Pöppel, Ernst 87, 99, 105, 144, 146, 149

Prantl, Heribert 65

Proust, Marcel 97

Reagan, Ronald 136

Ringer, Fritz K. 24, 143
Rizzollati, Giacomo 139f.

Rösler, Frank 34, 144

Roth, Gerhard 17, 20, 33, 35, 38f., 144, 146, 149

Rumpf, Horst 70

Schirrmacher, Frank 62

Schleiermacher, Friedrich Ernst

Daniel 24, 72

Schreglmann, Iska 105

Searle, John R. 10, 143

Senft, Holger 17, 141, 145

Singer, Tania 43, 144

Spinnagel, Christian 118

Spitz, René 41

Spitzer, Manfred 45, 60, 99, 108, 146

Spranger, Eduard 18

Straub, Eberhard 24f., 147

Stroebe, Wolfgang 14, 149

Thrun, Sebastian 114

Tomasello, Michael 10, 145

Treitschke, Heinrich von

Vermeer, Jan 134

Verplaetse, Jan 43, 48, 145

Wagner, Wolf 21, 149

Waitz, Georg 25

Weber, Max 46f., 72, 75

Weniger, Erich 16

Wieman, Carl 127-132, 150

Wolf, Maryanne 97f., 150

Wuketits, Franz M. 40, 68, 144

Zeki, Semir 134, 146

Ziegler, Jean 95, 146

Zierer, Klaus 107, 150 


\section{Sachregister}

Ära Adenauer 26

Altruistic Punishment 66, 69

Amygdala 135

Arienabende 24

Attachment 40-43, 48, 85, 88f., 95f., 120,126

Basalganglien (Striatum) 61, 33, 45 Belohnung(serwartungen) 45, 56 68, 88f., 112, 131 134, 138

Bertelsmann-Stiftung 106, 115 Betreuung und Beratung 21, 59, 63f., 125

Bildungskatastrophe 27 Bildungsrevolution 106 Bindungen, emotionale 41-43, 48 Bologna-Reform 21, 27, 63 Bundesassistentenkonferenz (BAK) 61

Catch-all-Reden (Vorlesungen) 79f., 91

Charisma(tisch) 42, 73, 75f., 78, 80, 82, 88, 90f., 120f., 134, 136f.

Deliberative Practice 132

Diskurs-Analyse 46

Dopamin 33, 42, 60, 137

Effortmechanismus 83

Embodied Learning 32, 48

Emotionen 12, 20, 32f., 36, 39, 44, $52,68,85,89$, 134f., $136 \mathrm{f}$.

Empathie 20, 43f., 48, 86, 120, 140f.

Empathie, intellektuelle und emotional 43, 90

Empathie, spielerische 44f., 48
Empathie, Vertrauensbeziehungen und "Altruistic Punishment" 69f.

Empirie, episodische und systematische 12, 91

Entertainment, digitales 107f., 120

False-Belief-Test $48-50$

Fernkommunikation 10f., 20, 31, 33, 121

Fishbowl-Verfahren 102f.

Fußballtraining, Automatismen 111

Gedächtnis, Arten von/Bildung von $35 f$.

Gedächtnis, emotionales 36, 133

Gedächtnis, Kurzzeit- (Arbeits-) 7, 37-39, 82

Gedächtnis, Langzeit- 7, 37-39

Gedächtnis, mangelnde Aufnahmeund Haltefähigkeit $82-85$

Gehirn, Beziehungsorgan 68

Gehirngerechtigkeit 7

Gehorsam 46f.

Geigenspiel 127

(Groß-)Hirnrinde 96f.

(Groß-)Hirnrinde, Anteriorer Cingulärer Cortex (ACC) 43, 140

(Groß-)Hirnrinde, Insula 43, 140

(Groß-)Hirnrinde, Okzipitallappen (= Hinterhauptlappen) 33, 96

(Groß-)Hirnrinde, Orbitofrontaler Cortex (OFC) 134, 138

(Groß-)Hirnrinde, Parietallappen (= Scheitellappen) 140

(Groß-)Hirnrinde, Stirnhirn 27, 33, $79,84,134,140$

(Groß-)Hirnrinde, Temporallappen (= Schläfenlappen) 33,96 
Hebb'sche Regel 37

Herrschaft 46

Hochschuldidaktik, Arbeitskreis für (AHD) 62, 102

Hochschuldidaktik, Deutsche Gesellschaft für (dghd) 62, 101

Hochschuldidaktik, mangelnde 61-63

Hochschuldidaktik, studentische Bewertungen 63

Hochschulen 10f., 14, 16 ,18, 20, 24, 26f., 32, 36, 52f., 55, 69, 71, $101,105,117,120-123,125$

Hochschulforum Digitalisierung $115,120,148$

Imitation, automatische 39

Imitationstheorie 48

Impuls-Reden $135 \mathrm{f}$.

Indifferenz, pädagogisch-didaktische 21, 23f., 29

Intentionalität, kollektive und subjektive 10,37

Inverted Classroom (Flipped Classroom) 101, 117f., 126

Jagdverhalten, neuro-motivationale Voraussetzungen 131f.

Kritische Universität 76

Lehre, Frontalismus 9

Lehre, obrigkeitliche 7, 12, 20f., 23-29, $125 f$.

Lehrpersonen 10, 14, 19f., 23, 42, $51,63,82,90,103,112,122$, $125 f ., 141$

Lehrprofessuren 120

Lehrveranstaltungen, Evaluation

12-15
Lehrvideos 110-112, 119

Leistungsprinzip 54-56

Lernbeziehungen, diskursabhängige 48-50

Lernen, bewusstes und unbewusstes $34 \mathrm{f}$.

Lernen, dialogisches 7, 21, 100-106

Lernen, dialogisches (Hauptmerkmale) 104

Lernen, digitales (Einübung von Automatismen) 110f.

Lernen, digitales (Fremdsprachen) 112

Lernen, digitales (Macro- und Micro-Lectures) 110

Lernen, digitales (mit Robotern) 108f.

Lernen, ertragreiches 20f., 41, 98 104, 115f., 120, 123, 126

Lernen, Frontalismus 7, 9, 16, 106, 119

Lernen, neurobiologische Grundlagen 21,32-39

Lernen, neuro-motivationale Voraussetzungen 127-132

Lernen, objektivistisch-empiristisches Modell 7, 16, 62

Lernen, spielerisches 44f., 107f.

Lernen, spurenarm und vielspurig/ netzübergreifend 96-98, 103f., 120,123

Lernen, synaptische Ebene 94-100

Lernen, Vergleich von Print-Texten und digitalen Texten $110 \mathrm{f}$.

Lern-Konstellation, asymmetrische 83f., 87 
Lernmaterialien, digitale 7,98, 106-116

Lesenlernen, neuronale Abläufe 96

Limbisches System 33f., 68, 134, 136,140

Macht, Machtbeziehungen 21, 46f.

Macht, Macht-Diskurs (= LernDiskurs) 52-58

Macht, instrumentelle und diskursive $46 f$.

Macht, kommunikative 47

Mental Framework 128, 130, 132

Moocs (Massive open online courses) 106, 113-115, 122

Multitasking $99 \mathrm{f}$.

Nahkommunikation 7, 10f., 17, 20, $31,70,119,121,125$

Nahkommunikation, Interview-

Format 93, 104-106

Nahkommunikation, privilegierte 64-66

Neugier, natürliche 100, 126

Neuroästhetik $133 \mathrm{f}$.

Neurobiologie, Herausforderungen 16f., 20

Neuroeconomics 68

Neuronalisationsanalyse 31

Ordinarienuniversität 24, 28, 61 , 71,102

Oxytozin 42, 89, 137

Pädagogik/Didaktik, schulbezogen $17 \mathrm{f}$.

Pädagogik/Didaktik, Notstand an

Hochschulen 17, 62f.

Pelzmantelkollegs 2, 4

Plagiatspraxis $66-70$
Poocs (Personal open online courses 106

Präsenz-Lernen 14, 93, 118-120, 126

Präsenz-Lernen, Einsatz digitaler Lernmaterialien 115-119

Predigten 137

Programm-Reden, Politik und Gesellschaft (Vergleich mit Vorlesungen) 132-139

Qualitätspakt Lehre 28

Recycling 97

Regisseur 19

Riechbeziehungen 33f.

Rituale 52-54

Rollenspiel 44f., 108

Rollenspiel, Vorlesungen 88f.

Schachspiel 83f., 127

Schaltkreise, neuronale 32-34

Schubkräfte, emotionale 7, 85-89, 114

SDS-Hochschuldenkschrift 1961 27f., 102

Serotonin 60f., 137

Shift from teaching to learning 13, 82, 101-103, 149

Spiegelneuronen 139-143

Sprechstunden $57 \mathrm{f}$.

Stifterverband für die deutsche Wissenschaft 28, 115

Studentenbewegung 76, 101

Symbolische Politik, Ruhigstellung $59 f$.

Thalamus 140

Theory of mind 140

Trauerreden $137 \mathrm{f}$.

Verfremdung, digitale 114 
Vorlesende Mandarine 71, 87

Vorlesungen, (Massen-) 81-85, 87, $89,114,128$

Vorlesungen, Impuls-Vorlesungen

81, 91f., 122, $133 \mathrm{f}$.

Vorlesungen, Instruktions-Vorlesun-

typ) 72, 81f., 89-91, 132-134

Vorlesungen, charismatische Faszination (formaler Idealtyp) 73

Vorlesungen, Infotainment (formaler Idealtyp) 74, 77-81, 90f.

Vorlesungen, snobistische Fachidiotie (formaler Idealtyp) 73, 77,80

Vorlesungen, Mischtyp 72, 80

Vorlesungs-Kritik 76-79

Vorlesungs-Lob $75 \mathrm{f}$.

Vorlesungsprinzip 15f., 23-26, 29, 38, 71f., 89-92

Wahlkampf-Reden 135

Winzig(keit) 22, 51, $57 f$.

Winzigkeit, Neurobiologie der 59-70, 125

Wissen, konzeptionelles 127-132

Wissenschaft, heilige 53f.

Wissenschaftsrat $27 \mathrm{f}$. 